\title{
Feedback Control Methods for a Single Machine Infinite Bus System
}

\author{
Pratik Vernekar, Zhongkui Wang, Andrea Serrani, and Kevin Passino \\ Department of Electrical and Computer Engineering, The Ohio State University \\ 2015 Neil Avenue, Columbus, OH 43210 \\ Email: pratik.vernekar@gmail.com, \\ wang.1231@osu.edu, \\ serrani.1@osu.edu, \\ passino.1@osu.edu
}

\begin{abstract}
In this manuscript, we present a high-fidelity physics-based truth model of a Single Machine Infinite Bus (SMIB) system. We also present reduced-order control-oriented nonlinear and linear models of a synchronous generator-turbine system connected to a power grid. The reduced-order control-oriented models are next used to design various control strategies such as: proportional-integral-derivative (PID), linear-quadratic regulator (LQR), pole placement-based state feedback, observer-based output feedback, loop transfer recovery (LTR)-based linear-quadratic-Gaussian (LQG), and nonlinear feedback-linearizing control for the SMIB system. The controllers developed are then validated on the high-fidelity physicsbased truth model of the SMIB system. Finally, a comparison is made of the performance of the controllers at different operating points of the SMIB system. The material presented in this manuscript is part of a course on "Control and Optimization for the Smart Grid" that was developed in the Electrical and Computer Engineering Department at the Ohio State University in 2011-2012. This project was funded by the U.S. Department of Energy.
\end{abstract}




\section{Contents}

1 A Single Generation Unit 4

2 Truth Model of the Synchronous Generator 5

2.1 Electrical Dynamics . . . . . . . . . . . . . . . . . . . . 6

2.1.1 Voltage Equation in the Static Frame . . . . . . . . . . . . . . 6

2.1.2 Voltage Equation in the Synchronously Rotating Frame . . . . . . . . . . . . . . . 8

2.1.3 Voltage Equation of the Synchronous Generator in Per Unit System . . . . . . . . . 10

2.2 A Synchronous Generator Connected to an Infinite Bus . . . . . . . . . . . . . . . . 10

2.3 Mechanical Dynamics: Swing Equation . . . . . . . . . . . . . . . . . . . 11

2.4 Truth Model of the Synchronous Generator . . . . . . . . . . . . . . . . . . . . . . . 14

2.5 Model of the Turbine-Governor System . . . . . . . . . . . . . . . . . . . . . . . 17

2.6 Truth model of the combined Synchronous Generator and Turbine-Governor System connected to an infinite bus . . . . . . . . . . . . . . . . . . . 18

2.7 Derivation of the Output Generator Terminal Voltage for the Truth Model _ . . . . . . . 18

3 The Reduced Order Simplified Model 20

3.1 Reduced order model of the combined Synchronous Generator and Turbine-Governor system connected to an infinite bus . . . . . . . . . . . . . . . . . . 20

3.2 Derivation of the Output Generator Terminal Voltage for the Reduced Order Model . . . . . 23

3.3 Linearization of the Reduced Order Model by Taylor series approximation . . . . . . . . . . . 25

3.4 Example . . . . . . . . . . . . . . . . . . . . . . . 30

4 Open Loop Input-Output Behavior of the Synchronous Generator and Turbine-Governor System

5 The Decoupled Reduced Order Model $\quad 41$

5.1 LFC Dynamics . . . . . . . . . . . . . . . . . . . . . . . . . . . . 42

5.1 .1 PID controller design for the LFC loop . . . . . . . . . . . . . . . . . . 46

5.2 AVR Dynamics . . . . . . . . . . . . . . . . . . . . . . . . 48

5.2 .1 PID controller design for the AVR loop . . . . . . . . . . . . . 50

5.3 LFC and AVR including coupling . . . . . . . . . . . . . . . . . . . . 52

6 PID Controller Design $\quad 53$

6.1 PID Controller Design based on linear model . . . . . . . . . . . . . . . . . . . 53

6.2 Simulation results for the PID Controllers applied to the Reduced Order Nonlinear Model . . 56

6.3 Simulation results for the PID Controllers applied to the Truth Model . . . . . . . . . . . . 59

7 Linear State-Space Controller Design $\quad 62$

7.1 State Feedback Controller design using LQR methodology . . . . . . . . . . . . . . . . . . 62

7.1.1 LQR Design based on linear model . . . . . . . . . . . . . . . . . . . 62

7.1.2 Simulation results for the Full-State Feedback LQR applied to the Reduced Order Nonlinear Model . . . . . . . . . . . . . . . . . . . . 65

7.1.3 Simulation results for the Full-State Feedback LQR applied to the Truth Model . . . . 69

7.2 State Feedback Controller design using Pole Placement Technique . . . . . . . . . . . . . . 72

7.2.1 Pole Placement Design based on linear model . . . . . . . . . . . . . . . . . . . 72

7.2.2 Simulation Results for the Full-State Feedback Pole Placement controller applied to the Reduced Order Nonlinear Model . . . . . . . . . . . . . . . . . . . . . . 75

7.2.3 Simulation Results for the Full-State Feedback Pole Placement controller applied to the Truth Model . . . . . . . . . . . . . . . . . . 78

7.3 Output Feedback Controller Design . . . . . . . . . . . . . . . . . . . . . 81 
7.3.1 Observer-based LQR Design based on linear model . . . . . . . . . . . . . . . . . . 81

7.3.2 Observer-Based Pole Placement Controller Design based on linear model . . . . . . . . 88

7.3.3 Simulation Results for the Observer-based LQR applied to the Reduced Order Nonlinear Model . . . . . . . . . . . . . . . . . . . . . 9 91

7.3.4 LTR-based LQG Controller applied to the Reduced Order Nonlinear Model . . . . . . 93

7.3.5 LTR-based LQG Controller applied to the Truth Model . . . . . . . . . . . . . . . . 97

8 Nonlinear Feedback Linearizing Controller Design $\quad 100$

8.1 Nonlinear Feedback Linearizing Controller Design for the Reduced Order Model . . . . . . . . 102

8.2 Simulation Results for the Nonlinear Feedback Linearizing Controller applied to the Truth

Model . . . . . . . . . . . . . . . . . . . . . . 109

9 Simulation results for the Controllers at different Operating Points 114

9.1 Simulation results for the Controllers at Operating Point II . . . . . . . . . . . . . . . . . . . 115

9.2 Simulation results for the Controllers at Operating Point III . . . . . . . . . . . . . . . . . 119

10 Appendix 124

10.1 Derivation of Park's Transformation . . . . . . . . . . . . . . . . . . . . . . 124

10.1.1 Stator and Rotor Inductances . . . . . . . . . . . . . . . . . . . . . 124

10.1 .2 Park's Transformation . . . . . . . . . . . . . . . . . 126

10.1.3 Voltage Equation in the Rotating Frame . . . . . . . . . . . . . . . . 127

10.2 Per Unit Conversion . . . . . . . . . . . . . . . . . . . . . . . 130

10.2.1 Choosing a base for stator quantities . . . . . . . . . . . . . 131

10.2.2 Choosing a base for rotor quantities . . . . . . . . . . . . . . 131

10.2.3 The correspondence of per unit stator EMF to rotor quantities . . . . . . . . . . 133

10.3 Sub-transient and Transient Inductances and Time Constants . . . . . . . . . . . . . . . 133

10.3.1 Sub-transient and Transient Inductances . . . . . . . . . . . . . . . . . 133

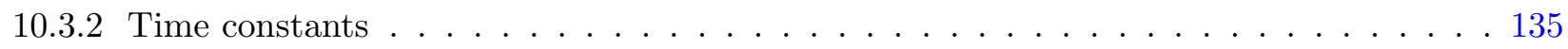

10.4 Simplified Model of the Synchronous Generator . . . . . . . . . . . . . . . . 136

10.4 .1 The two-axis model . . . . . . . . . . . . . . . . . . 137

10.4.2 The one-axis or the third-order simplified model . . . . . . . . . . . . . . 143 


\section{A Single Generation Unit}

Fossil fuels such as coal, oil, and natural gas have been the main resources of electrical energy for many years. However in recent years, there has been a gradual increase in the use of renewable energy resources for electricity generation, such as hydro, biogas, solar, wind, and geothermal energy. Electricity generation is basically the process of generating electric energy from other forms of energy. An electromechanical device called synchronous generator driven by a prime mover, usually a turbine or a diesel engine, converts the mechanical energy into alternating current (AC) electrical energy.

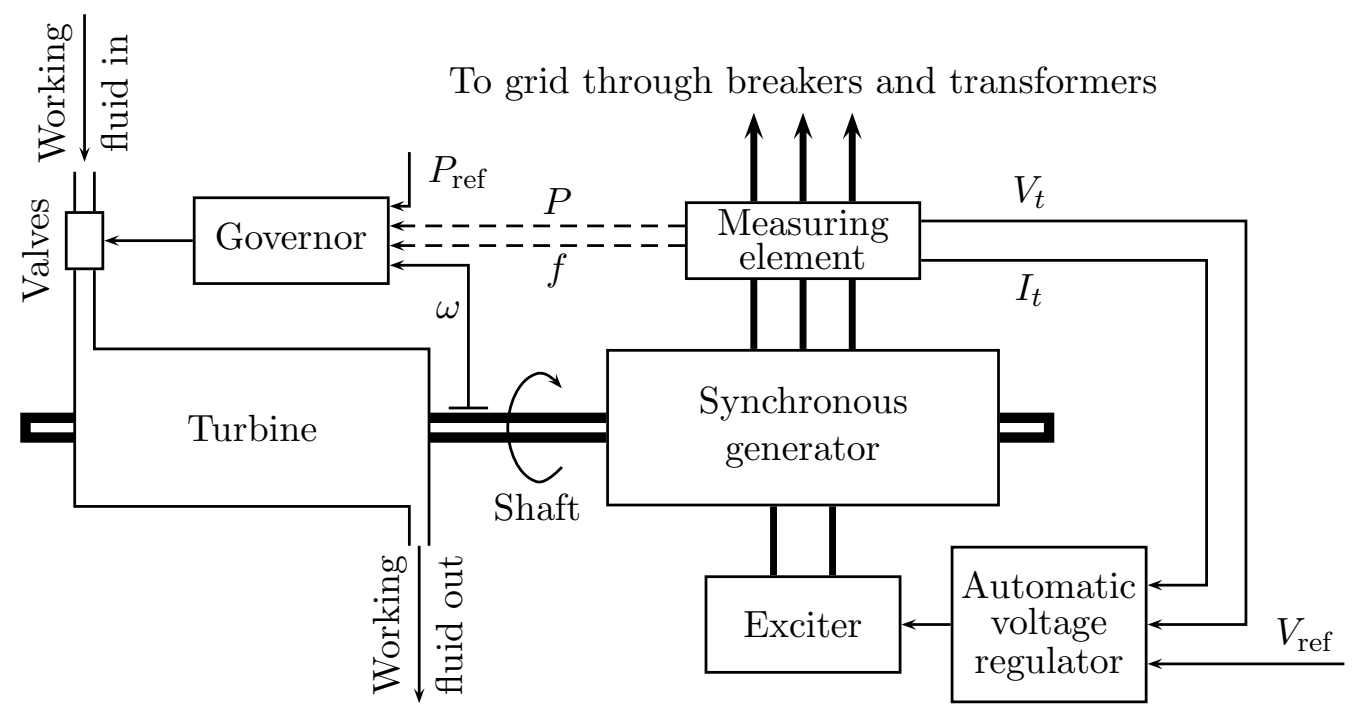

Figure 1.1: Structure of a generation unit.

The system shown in Figure 1.1 is a general structure of a single generation unit [7]. The turbine extracts the energy from the working fluid flowing into the turbine through valves. Typical working fluids are gas, steam, and water. The shaft is the rotary part of the turbine on which the synchronous generator is mounted. The opening and closing of the turbine valves or the frequency at which the turbine valves operate is regulated to a reference frequency of $f_{\text {ref }}$, by a turbine governor. The frequency of the grid $f$ which is measured by the measuring element is directly related to the output power $P$. Thus, the output of the synchronous generator $P$ and the angular frequency of the shaft $\omega$ are measured and fed back to the governor by the measuring element. Meanwhile, the measuring element also provides information about the output terminal voltage $V_{t}$ and output current $I_{t}$ of the synchronous generator to the automatic voltage regulator (AVR), which is able to control the terminal voltage of the synchronous generator to a reference voltage $V_{\text {ref }}$ through the exciter. The excitation current generated by the exciter produces the magnetic field inside the generator.

Thus, from the above figure we can see that in an interconnected power system, where a synchronous generator is connected to a grid, load frequency control (LFC) and automatic voltage regulator (AVR) equipment is installed for each generator. Figure 1.1 shows two control loops, namely the load frequency control (LFC) loop and the automatic voltage regulator (AVR) loop. The controllers are set for a particular operating condition and accommodate small changes in load demand to maintain the frequency and voltage magnitude within the specified limits. Small changes in real power are mainly dependent on changes in rotor angle $\delta$, and thus the frequency $\omega$. The reactive power is mainly dependent on the voltage magnitude (i.e., on the generator excitation). The excitation system time constant which is an indication of how fast the transients of the AVR loop decay exponential to zero, is much smaller than the prime mover time constant. Thus the transients of the excitation system and thus the AVR loop decay much faster than the transients of the LFC loop, hence it does not affect the LFC dynamics. Thus, the cross-coupling between the LFC loop and the AVR loop is negligible. Hence, load frequency control and excitation voltage control are usually analyzed independently [8]. 
The operation objectives of the LFC are to maintain reasonably uniform frequency, and to divide the load between generators [8]. The change in frequency is sensed, which is a measure of the change in rotor angle $\delta$, i.e., the error $\Delta \delta$ to be corrected. The error signal i.e., $\Delta f=f_{\text {ref }}-f$ is amplified, mixed, and transformed into a real power command signal $\Delta P_{V}=P_{\text {ref }}-P$, which is sent to the prime mover to call for an increment in the torque. The prime mover, therefore, brings about a change in the generator output which will change the value of $\Delta f$ within the specified tolerance.

The generator excitation system maintains the generator terminal voltage and controls the reactive power flow. The generator excitation of older systems may be provided through slip rings and brushes by means of DC generators mounted on the same shaft as the rotor of the synchronous machine. However, modern excitation systems also known as brush-less excitation systems, usually use AC generators with rotating rectifiers. The sources of reactive power are generators, capacitors, and reactors. The generator reactive power is controlled by field excitation using the AVR. The role of an AVR is to hold the terminal voltage magnitude of a synchronous generator at a specified level. An increase in the reactive power load of the generator is accompanied by a drop in the terminal voltage magnitude. The voltage magnitude is sensed through a potential transformer on one phase. This voltage is rectified and compared to a DC set point signal. The amplified error signal controls the exciter field and increases the exciter terminal voltage. Thus, the generator field current is increased, which results in an increase in the generated electromotiveforce (emf). The reactive power generation is increased to a new equilibrium, raising the terminal voltage to the desired value.

\section{Truth Model of the Synchronous Generator}

In the previous section we saw the basic working of a single generation unit and the respective roles of the load frequency control and the automatic voltage regulator. In this section we will derive the truth model of a synchronous generator. Before we proceed to the derivation of the truth model, we present some preliminaries about the synchronous generator.

The two main parts of a synchronous generator can be described in either electrical or mechanical terms:

- Electrical:

- Armature: The power-producing component of an electrical machine. In a synchronous generator, the armature windings generate the electric current. The armature can be on either the rotor or the stator.

- Field: The magnetic field component of an electrical machine. The magnetic field of the synchronous generator can be provided by either electromagnets or permanent magnets mounted on either the rotor or the stator.

- Mechanical:

- Rotor: The rotating part of the synchronous generator.

- Stator: The stationary part of the synchronous generator.

Because power transferred into the field circuit is much smaller than in the armature circuit, AC generators always have the field winding on the rotor and the stator has the armature winding. Thus, a classical synchronous generator has two main magnetic parts: the stator and the rotor, as shown in Figure 2.1. The windings are represented by one-turn coils, specifically, the small circles " $\bigcirc$ " in the figure. The black dots and the crosses inside the small circles indicate the directions of the currents flowing in the windings, i.e., " $\times$ " means the current flowing in the direction from the outside of the paper vertically into the paper and "•" means the current flowing from the inside of the paper to the outside. 


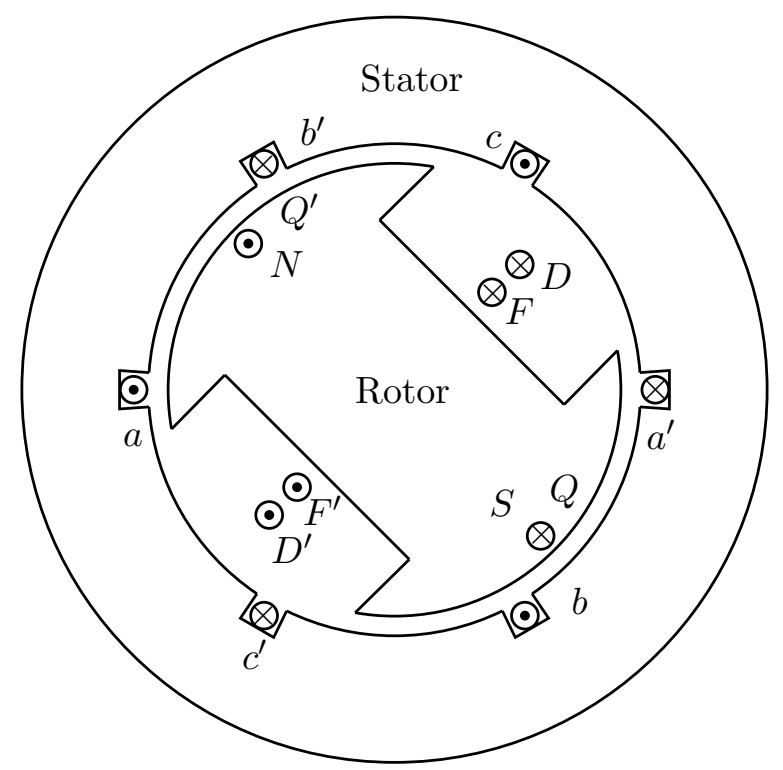

Figure 2.1: Schematic structure of the synchronous generator.

The armature winding, which carries the load current $I_{t}$ and supplies power to the grid, is placed in equidistant slots on the inner surface of the stator and consists of three identical phase windings, namely, $a a^{\prime}$, $b b^{\prime}$ and $c c^{\prime}$. The rotor is mounted on the shaft through which the synchronous generator is driven by the prime mover, for instance, a hydro turbine. The rotor consists of two poles, $N$ pole and $S$ pole, as seen in Figure 2.1. The direct current (DC) excitation winding represented by $F F^{\prime}$ is wrapped around the rotor. From basic physics, we know that the DC flowing in the excitation winding generates a magnetic flux. Magnetic flux is a measure of the amount of magnetic field (also called magnetic flux density) passing through a given surface (such as a conducting coil). The SI unit of magnetic flux is the weber (in derived units: volt-seconds). The strength of the magnetic flux generated is proportional to the excitation current and its direction is known by using the right-hand rule. As the rotor rotates, the magnetic flux generated by the excitation winding wrapped on the rotor changes spatially. Thus, there are magnetic flux changes in the armature windings as a result of which an emf is induced in each phase of the three-phase stator armature winding. By connecting armature windings to the grid, a closed-loop circuit is formed which allows the AC to flow from the synchronous generator to the grid. The AC armature currents produce their own armature reaction magnetic flux which is of constant magnitude but rotates at the same speed as the rotor. The excitation flux and the armature reaction flux then produce a resultant flux that is stationary with respect to the rotor. Two other windings represented by $D D^{\prime}$ and $Q Q^{\prime}$ are the two short-circuit damper (or, amortisseur) windings which help to damp the mechanical oscillations of the rotor [2]. Hence, two dynamics will characterize the generator, i.e., electrical dynamics and mechanical dynamics.

\subsection{Electrical Dynamics}

In this section we present the equations governing the electrical dynamics of a synchronous generator which are described in [2]. We first present the voltage equations of a synchronous generator in the static frame, and then use Park's transformation to convert these to the rotating frame.

\subsubsection{Voltage Equation in the Static Frame}

In this subsection we present the voltage equations in the static frame. The static frame contains three reference axes $a, b$, and $c$ which correspond to the three armature windings on the stator. Before presenting the details of the voltage equation of a synchronous generator, we start by considering the general case of a set of coupled coils in which one or more of the coils is mounted on a shaft and can rotate. The situation is shown schematically in Figure 2.2. 


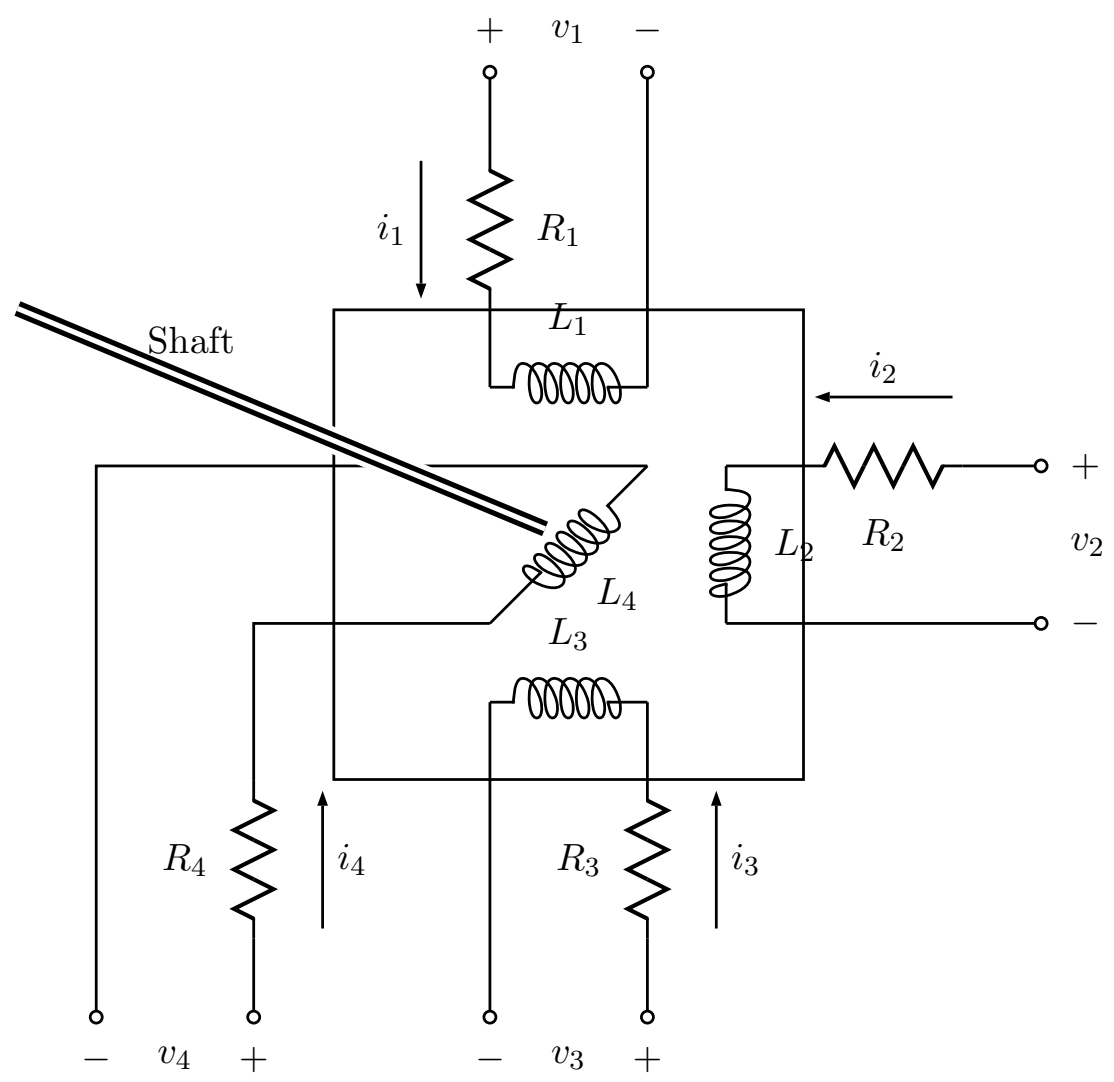

Figure 2.2: Coupled coils.

Assume that for any fixed shaft angle $\theta$ there is a linear relationship between the flux linkage $\lambda$ and current $i$. Flux linkage is defined as the total flux passing through a surface (i.e. normal to that surface) formed by a closed conducting loop. Thus we get the relationship $\boldsymbol{\lambda}=\mathbf{L}(\theta) \mathbf{i}$, where, in the case of Figure 2.2 , i and $\boldsymbol{\lambda}$ are $4 \times 1$ vectors, and $\mathbf{L}$ is a $4 \times 4$ matrix. By applying Kirchhoff's voltage law (KVL) to the circuit in Figure 2.2, we have

$$
\mathbf{v}=\mathbf{R i}+\frac{d \boldsymbol{\lambda}}{d t}
$$

where $\mathbf{R}$ is a $4 \times 4$ matrix. Equation (2.1) indicates that the terminal voltage of each coil equals the sum of the voltage drop on the resistance and the derivative of the flux linkage. 


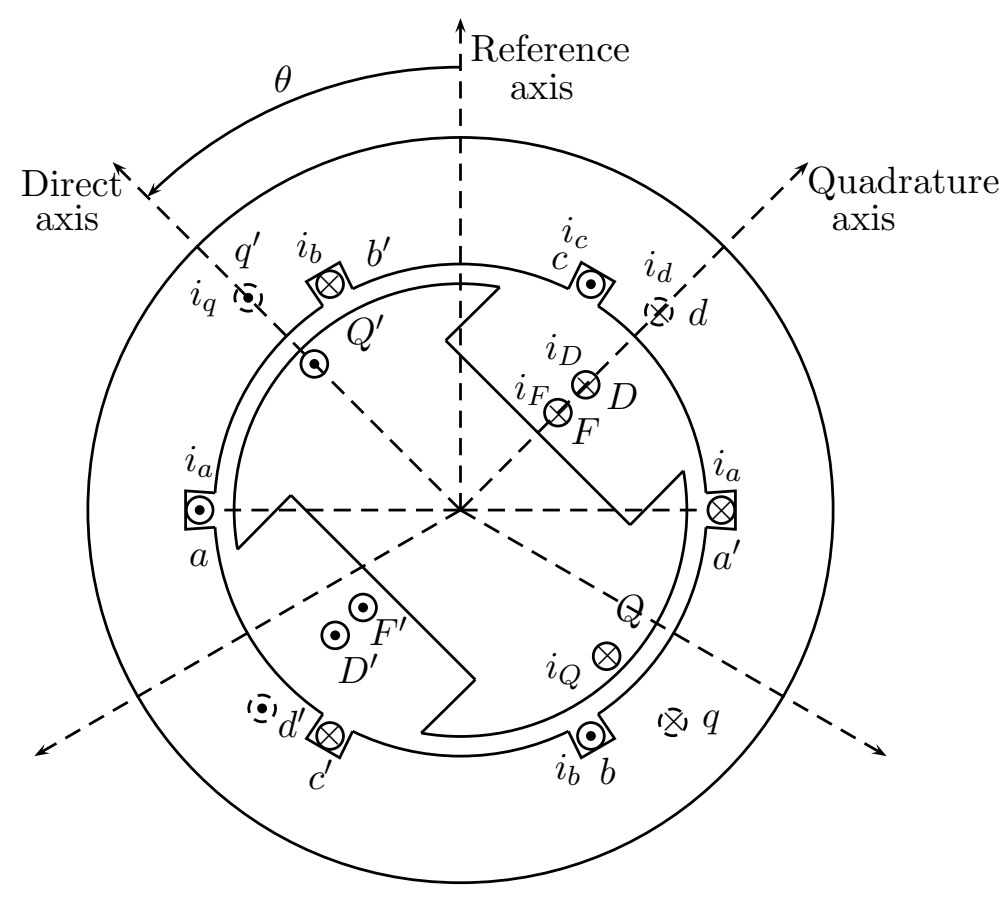

Figure 2.3: Machine schematic.

By applying Equation (2.1) and using the circuits convention on the associated reference directions in Figure 2.3, we get the relationship between voltages, currents, and flux linkages [2].

$$
\begin{aligned}
{\left[\begin{array}{c}
v_{a^{\prime} a} \\
v_{b^{\prime} b} \\
v_{c^{\prime} c} \\
v_{F F^{\prime}} \\
v_{D D^{\prime}} \\
v_{Q Q^{\prime}}
\end{array}\right] } & =\left[\begin{array}{cccccc}
r & & & & & \\
& r & & & 0 & \\
& r & & & \\
& & r_{F} & & \\
0 & & & r_{D} & \\
& & & & r_{Q}
\end{array}\right]\left[\begin{array}{c}
i_{a} \\
i_{b} \\
i_{c} \\
i_{F} \\
i_{D} \\
i_{Q}
\end{array}\right]+\frac{d}{d t}\left[\begin{array}{c}
\lambda_{a a^{\prime}} \\
\lambda_{b b^{\prime}} \\
\lambda_{c c^{\prime}} \\
\lambda_{F F^{\prime}} \\
\lambda_{D D^{\prime}} \\
\lambda_{Q Q^{\prime}}
\end{array}\right] \\
& =\mathbf{R i}+\frac{d \boldsymbol{\lambda}}{d t}
\end{aligned}
$$

We simplify the equation above by using a single-subscript notation, i.e., $v_{a} \triangleq v_{a a^{\prime}}=-v_{a^{\prime} a}, v_{b} \triangleq v_{b b^{\prime}}=-v_{b^{\prime} b}$, $v_{c} \triangleq v_{c c^{\prime}}, v_{F} \triangleq v_{F F^{\prime}}, v_{D} \triangleq v_{D D^{\prime}}$, and $v_{Q} \triangleq v_{Q Q^{\prime}}$. Here, we define $\mathbf{v} \triangleq\left[v_{a}, v_{b}, v_{c},-v_{F},-v_{D},-v_{Q}\right]^{\mathrm{T}}$ to be the voltage vector consisting of the three phase terminal voltages $\left(v_{a}, v_{b}, v_{c}\right)$, and the voltage of the field winding $\left(v_{F}\right)$ and two damper windings $\left(v_{D}, v_{Q}\right)$. The corresponding current vector is defined as $\mathbf{i} \triangleq\left[i_{a}, i_{b}, i_{c}, i_{F}, i_{D}, i_{Q}\right]^{\mathrm{T}}$. Then Equation $(2.2)$ can be written as follows:

$$
\mathbf{v}=-\mathbf{R i}-\frac{d \boldsymbol{\lambda}}{d t}
$$

\subsubsection{Voltage Equation in the Synchronously Rotating Frame}

The electrical dynamics as given in Equation (2.2) are derived in the static abc frame. The flux linkage in Equation (2.2) is dependent on the self and mutual inductances which are not constant, but are time varying. In the voltage equation as given in Equation (2.3) the $\dot{\lambda}$ term must be computed as $\dot{\lambda}=L \dot{i}+\dot{L} i$. Thus, to simplify the equations we make a coordinate transformation which transforms variables from the $a b c$ static frame to a synchronously rotating frame (which is also called $d q$ frame, see Figure 2.3). As a result 
of this transformation we introduce two fictitious windings $d d^{\prime}$ and $q q^{\prime}$, as shown in Figure 2.3. Thus we get

$$
\begin{aligned}
& v_{d}=-r i_{d}-\omega \lambda_{q}-\frac{d \lambda_{d}}{d t} \\
& v_{q}=-r i_{q}+\omega \lambda_{d}-\frac{d \lambda_{q}}{d t} \\
& v_{F}=r_{F} i_{F}+\frac{d \lambda_{F}}{d t} \\
& v_{D}=r_{D} i_{D}+\frac{d \lambda_{D}}{d t} \\
& v_{Q}=r_{Q} i_{Q}+\frac{d \lambda_{Q}}{d t}
\end{aligned}
$$

The details of the derivation of this transformation are given in the Appendix. The extra terms $-\omega \lambda_{q}$ and $\omega \lambda_{d}$ are introduced by the transformation.

We can rearrange Equation (2.4) to put the quantities on the direct axis together and the quantities on the quadrature axis together. Hence, Equation (2.4) is rewritten as follows:

$$
\begin{aligned}
& v_{d}=-r i_{d}-\omega \lambda_{q}-\frac{d \lambda_{d}}{d t} \\
& v_{F}=r_{F} i_{F}+\frac{d \lambda_{F}}{d t} \\
& v_{D}=r_{D} i_{D}+\frac{d \lambda_{D}}{d t} \\
& v_{q}=-r i_{q}+\omega \lambda_{d}-\frac{d \lambda_{q}}{d t} \\
& v_{Q}=r_{Q} i_{Q}+\frac{d \lambda_{Q}}{d t}
\end{aligned}
$$

As the damper windings are short-circuited, the terminal voltages are both zero. As shown in Figure 2.3 the direct axis is perpendicular to the windings $d d^{\prime}, F F^{\prime}$, and $D D^{\prime}$; the quadrature axis is perpendicular to the windings $q q^{\prime}$ and $Q Q^{\prime}$. Using the right hand thumb rule we can see that the flux linkage due to the currents $i_{d}, i_{F}$, and $i_{D}$ is along the direct axis and the flux linkage due to the currents $i_{q}$ and $i_{Q}$ is along the quadrature axis. Thus, the flux linkage $\lambda_{d}$ along the $d d^{\prime}$ winding depends on the currents $i_{d}, i_{F}$, and $i_{D}$ and is given by $\lambda_{d}=L_{d} i_{d}+k M_{F} i_{F}+k M_{D} i_{D}$, where $L_{d}$ is the self inductance of $d d^{\prime}$ winding, $M_{F}$ is the mutual inductance between $d d^{\prime}$ and $F F^{\prime}$ windings, and $M_{D}$ is the mutual inductance between $d d^{\prime}$ and $D D^{\prime}$ windings, respectively. We can derive equations for $\lambda_{F}$ and $\lambda_{D}$ in a similar fashion. Also, the flux linkage $\lambda_{q}$ along the $q q^{\prime}$ winding depends on the currents $i_{q}$, and $i_{Q}$ and is given by $\lambda_{q}=L_{q} i_{q}+k M_{Q} i_{Q}$, where $L_{q}$ is the self inductance of the $q q^{\prime}$ winding, and $M_{Q}$ is the mutual inductance between $q q^{\prime}$ and $Q Q^{\prime}$ windings, respectively. We can derive $\lambda_{Q}$ using the same approach. Also note that in Equation (2.6) and Equation (2.7) which gives a relationship between the flux and the current in each winding, the mutual inductance between the windings $F F^{\prime}$ and $D D^{\prime}$ is denoted by $M_{R}$, and self-inductances of the windings are denoted by $L_{d}, L_{F}, L_{D}, L_{q}$, and $L_{Q}$, respectively. Thus, the connection between the flux and the current is given by

$$
\left[\begin{array}{c}
\lambda_{d} \\
\lambda_{F} \\
\lambda_{D}
\end{array}\right]=\left[\begin{array}{ccc}
L_{d} & k M_{F} & k M_{D} \\
k M_{F} & L_{F} & M_{R} \\
k M_{D} & M_{R} & L_{D}
\end{array}\right]\left[\begin{array}{c}
i_{d} \\
i_{F} \\
i_{D}
\end{array}\right]
$$

and

$$
\left[\begin{array}{c}
\lambda_{q} \\
\lambda_{Q}
\end{array}\right]=\left[\begin{array}{cc}
L_{q} & k M_{Q} \\
k M_{Q} & L_{Q}
\end{array}\right]\left[\begin{array}{c}
i_{q} \\
i_{Q}
\end{array}\right]
$$

where $k=\sqrt{3 / 2}$. If we substitute Equation (2.6) and Equation (2.7) into Equation (2.5) and put it in 
matrix form, we obtain

$$
\left[\begin{array}{c}
v_{d} \\
v_{F} \\
0 \\
v_{q} \\
0
\end{array}\right]=\left[\begin{array}{ccccc}
-r & 0 & 0 & -\omega L_{q} & -\omega k M_{Q} \\
0 & r_{F} & 0 & 0 & 0 \\
0 & 0 & r_{D} & 0 & 0 \\
\omega L_{d} & \omega k M_{F} & \omega k M_{D} & -r & 0 \\
0 & 0 & 0 & 0 & r_{Q}
\end{array}\right]\left[\begin{array}{c}
i_{d} \\
i_{F} \\
i_{D} \\
i_{q} \\
i_{Q}
\end{array}\right]+\left[\begin{array}{ccccc}
-L_{d} & -k M_{F} & -k M_{D} & 0 & 0 \\
k M_{F} & L_{F} & M_{R} & 0 & 0 \\
k M_{D} & M_{R} & L_{D} & 0 & 0 \\
0 & 0 & 0 & -L_{q} & -k M_{Q} \\
0 & 0 & 0 & k M_{Q} & L_{Q}
\end{array}\right]\left[\begin{array}{c}
\dot{i_{d}} \\
\dot{i}_{F} \\
\dot{i_{D}} \\
\dot{i_{q}} \\
\dot{i_{Q}}
\end{array}\right]
$$

Moving the derivative of the current to the left-hand side, we obtain

$$
\left[\begin{array}{ccccc}
L_{d} & k M_{F} & k M_{D} & 0 & 0 \\
-k M_{F} & -L_{F} & -M_{R} & 0 & 0 \\
-k M_{D} & -M_{R} & -L_{D} & 0 & 0 \\
0 & 0 & 0 & L_{q} & k M_{Q} \\
0 & 0 & 0 & -k M_{Q} & -L_{Q}
\end{array}\right]\left[\begin{array}{c}
\dot{i_{d}} \\
\dot{i_{F}} \\
\dot{i_{D}} \\
\dot{i_{q}} \\
\dot{i_{Q}}
\end{array}\right]=\left[\begin{array}{ccccc}
-r & 0 & 0 & -\omega L_{q} & -\omega k M_{Q} \\
0 & r_{F} & 0 & 0 & 0 \\
0 & 0 & r_{D} & 0 & 0 \\
\omega L_{d} & \omega k M_{F} & \omega k M_{D} & -r & 0 \\
0 & 0 & 0 & 0 & r_{Q}
\end{array}\right]\left[\begin{array}{c}
i_{d} \\
i_{F} \\
i_{D} \\
i_{q} \\
i_{Q}
\end{array}\right]-\left[\begin{array}{c}
v_{d} \\
v_{F} \\
0 \\
v_{q} \\
0
\end{array}\right]
$$

\subsubsection{Voltage Equation of the Synchronous Generator in Per Unit System}

A normalization of variables called the per unit normalization is always desirable. The idea is to pick base values for quantities such as voltages, currents, impedances, power, and so on, and to define the quantity in per unit as

$$
\text { quantity in per unit }=\frac{\text { actual quantity }}{\text { base value of quantity }}
$$

By carefully choosing the base quantities for both stator and rotor variables, the electrical dynamics expressed by Equation (2.9) can be expressed in the p.u. system as

$$
\left[\begin{array}{ccccc}
L_{d} & k M_{F} & k M_{D} & 0 & 0 \\
-k M_{F} & -L_{F} & -M_{R} & 0 & 0 \\
-k M_{D} & -M_{R} & -L_{D} & 0 & 0 \\
0 & 0 & 0 & L_{q} & k M_{Q} \\
0 & 0 & 0 & -k M_{Q} & -L_{Q}
\end{array}\right]\left[\begin{array}{c}
\dot{i}_{d} \\
\dot{i}_{F} \\
\dot{i_{D}} \\
\dot{i_{q}} \\
\dot{i_{Q}}
\end{array}\right]=\left[\begin{array}{ccccc}
-r & 0 & 0 & -\omega L_{q} & -\omega k M_{Q} \\
0 & r_{F} & 0 & 0 & 0 \\
0 & 0 & r_{D} & 0 & 0 \\
\omega L_{d} & \omega k M_{F} & \omega k M_{D} & -r & 0 \\
0 & 0 & 0 & 0 & r_{Q}
\end{array}\right]\left[\begin{array}{c}
i_{d} \\
i_{F} \\
i_{D} \\
i_{q} \\
i_{Q}
\end{array}\right]-\left[\begin{array}{c}
v_{d} \\
v_{F} \\
0 \\
v_{q} \\
0
\end{array}\right] \text { p.u. }
$$

It is obvious that Equation (2.9) and Equation (2.11) are identical. This is always possible if base quantities are carefully chosen. The derivation of Equation (2.11) can be found in [1].

\subsection{A Synchronous Generator Connected to an Infinite Bus}

A typical configuration of a generation system model is a synchronous generator connected to an infinite bus as shown in Figure 2.4. The figure shows a synchronous generator connected to an infinite bus through a transmission line having resistance $R_{e}$ and inductance $L_{e}$. Only the voltages and currents for phase $a$ are shown, where $v_{a}$ is the phase voltage, $i_{a}$ is the phase current, and $V_{\infty}$ is the infinite bus voltage. An infinite bus is an approximation of a large interconnected power system, where the action of a single generator will not affect the operation of the power grid. In an infinite bus, the system frequency is constant, independent of power flow, and the system voltage is constant, independent of reactive power consumed or supplied. 


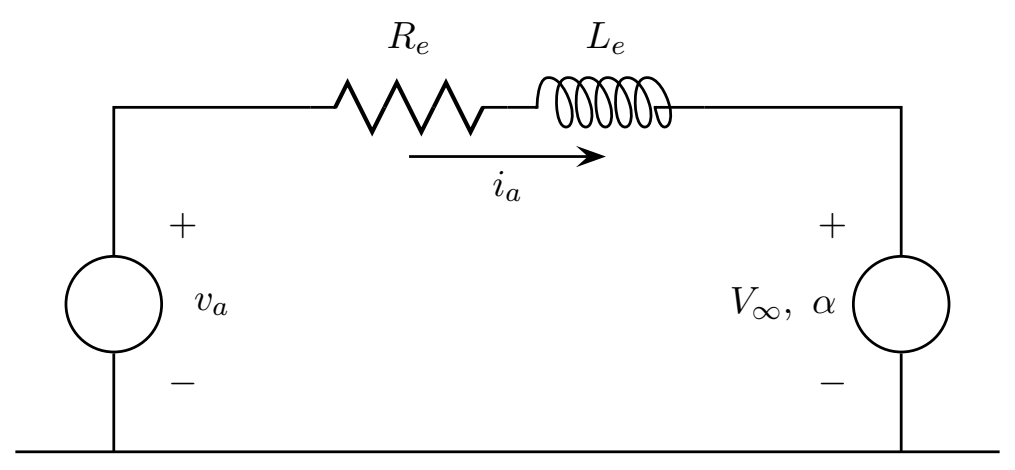

Figure 2.4: Synchronous generator loaded by an infinite bus.

The constraints of the infinite bus are given by

$$
\left[\begin{array}{l}
v_{d} \\
v_{q}
\end{array}\right]=R_{e}\left[\begin{array}{l}
i_{d} \\
i_{q}
\end{array}\right]+L_{e}\left[\begin{array}{c}
\dot{i}_{d} \\
\dot{i}_{q}
\end{array}\right]-\omega L_{e}\left[\begin{array}{c}
-i_{q} \\
i_{d}
\end{array}\right]+\sqrt{3} V_{\infty}\left[\begin{array}{c}
-\sin (\delta-\alpha) \\
\cos (\delta-\alpha)
\end{array}\right]
$$

By including Equation (2.12), we can rewrite Equation (2.11) as

$$
\begin{aligned}
{\left[\begin{array}{ccccc}
L_{d}+L_{e} & k M_{F} & k M_{D} & 0 & 0 \\
-k M_{F} & -L_{F} & -M_{R} & 0 & 0 \\
-k M_{D} & -M_{R} & -L_{D} & 0 & 0 \\
0 & 0 & 0 & L_{q}+L_{e} & k M_{Q} \\
0 & 0 & 0 & -k M_{Q} & -L_{Q}
\end{array}\right]\left[\begin{array}{c}
\dot{i_{d}} \\
\dot{i_{F}} \\
\dot{i_{D}} \\
\dot{i_{q}} \\
\dot{i_{Q}}
\end{array}\right] } & =\left[\begin{array}{ccccc}
-r-R_{e} & 0 & 0 & -\omega\left(L_{q}+L_{e}\right) & -\omega k M_{Q} \\
0 & r_{F} & 0 & 0 & 0 \\
0 & 0 & r_{D} & 0 & 0 \\
\omega\left(L_{d}+L_{e}\right) & \omega k M_{F} & \omega k M_{D} & -r-R_{e} & 0 \\
0 & 0 & 0 & r_{Q}
\end{array}\right]\left[\begin{array}{c}
i_{d} \\
i_{F} \\
i_{D} \\
i_{q} \\
i_{Q}
\end{array}\right] \\
& -\left[\begin{array}{c}
0 \\
v_{F} \\
0 \\
0 \\
0
\end{array}\right]-\sqrt{3} V_{\infty}\left[\begin{array}{c}
-\sin (\delta-\alpha) \\
0 \\
0 \\
\cos (\delta-\alpha) \\
0
\end{array}\right]
\end{aligned}
$$

Thus, Equation (2.13) describes the electrical dynamics of a synchronous generator connected to an infinite bus.

\subsection{Mechanical Dynamics: Swing Equation}

In this subsection, we present the mechanical dynamics of the synchronous generator. Under normal operating conditions, the relative position of the rotor axis and the resultant magnetic field axis is fixed. The angle between the two is known as the power angle or torque angle. During any disturbance, the rotor will decelerate or accelerate with respect to the synchronously rotating air gap magneto-motive force (mmf), which is any physical driving (motive) force that produces magnetic flux, and a relative motion begins. In this context, the expression 'driving force' is used in a general sense of work potential, and is analogous, but distinct, from force measured in Newton's. In magnetic circuits the magneto-motive force (mmf) plays a role analogous to the role emf (voltage) plays in electric circuits. The equation describing this relative motion is known as the swing equation [1]. If, after this oscillatory period, the rotor locks back into synchronous speed, the generator will maintain its stability. If the disturbance does not involve any net change in power, the rotor returns to its original position. If the disturbance is created by a change in generation, load, or in network conditions, the rotor comes to a new operating power angle relative to the synchronously revolving field.

The swing equation thus governs the motion of the machine rotor relating the moment of inertia (also referred to as the rotational inertia of the rotor) to the resultant of the mechanical and electrical torques on the rotor, i.e., $J \ddot{\theta}=T_{a} \mathrm{~N} \cdot \mathrm{m}$, where $J$ is the moment of inertia of all rotating masses attached to the 
shaft, $\theta$ is the mechanical angle of the shaft with respect to a fixed reference, and $T_{a}$ is the accelerating torque acting on the shaft. The torque is given by $T_{a}=T_{m}-T_{e}-T_{d}$, where $T_{m}, T_{e}$, and $T_{d}$ are mechanical, electrical, and damping torques, respectively. The mechanical torque $T_{m}$ is the driving torque provided by the prime mover. The electrical torque $T_{e}$ is generated by the load currents of the armature windings on the stator. The damping torque $T_{d}$ is produced by the damper windings on the rotor. The angular reference may be chosen relative to a synchronously rotating reference frame moving with constant angular velocity $\omega_{m R}$. The rotor angle in the static frame is given by $\theta(t)=\left(\omega_{m R} t+\beta\right)+\delta_{m}$, where $\beta$ is a constant and $\delta_{m}$ is the rotor position also referred to as the mechanical torque angle, measured from the synchronously rotating reference frame. Let us denote the shaft angular velocity in the static frame as $\omega_{m}$ in rad/sec, thus we have $\omega_{m}=\dot{\theta}=\omega_{m R}+\dot{\delta}_{m}$. By taking the derivative of $\omega_{m}$ and second derivative of $\theta$ we obtain $\dot{\omega}_{m}=\ddot{\theta}=\ddot{\delta}_{m}$, if we substitute this in $J \ddot{\theta}=T_{a}$ we have

$$
J \ddot{\theta}=J \ddot{\delta}_{m}=J \dot{\omega}_{m}=T_{a}=T_{m}-T_{e}-T_{d}[\mathrm{~N} \cdot \mathrm{m}]
$$

The product of torque and angular velocity is the shaft power in watts, thus we have

$$
J \ddot{\delta}_{m} \omega_{m}=P_{m}-P_{e}-P_{d}[\mathrm{~W}]
$$

The quantity $J \omega_{m}$ is called the inertia constant and is denoted by $M$. It is related to the kinetic energy of the rotating masses $W_{k}$, where $W_{k}=\frac{1}{2} J \omega_{m}^{2}$. M is computed as

$$
M=J \omega_{m}=\frac{2 W_{k}}{\omega_{m}}[\mathrm{~J} \cdot \mathrm{s}]
$$

Although $M$ is called an inertia constant, it is not really constant when the rotor speed deviates from the synchronous speed $\omega_{m R}$. However, since $\omega_{m}$ does not change by a large amount before stability is lost, $M$ is evaluated at the synchronous speed $\omega_{m R}$ and is considered to remain constant, i.e.,

$$
M=J \omega_{m} \cong \frac{2 W_{k}}{\omega_{m R}}[\mathrm{~J} \cdot \mathrm{s}]
$$

The swing equation in terms of the inertia constant becomes

$$
M \ddot{\delta}_{m}=M \dot{\omega}_{m}=P_{m}-P_{e}-P_{d}
$$

In relating the machine inertial performance to the network, it would be more useful to write Equation (2.18) in terms of an electrical angle that can be conveniently related to the position of the rotor. Such an angle is the torque angle $\delta$, which is the angle between the magneto-motive force (mmf) and the resultant magnetomotive force $(\mathrm{mmf})$ in the air gap, both rotating at synchronous speed. It is also the electrical angle between the generated emf and the resultant stator voltage phasors. The torque angle $\delta$, which is the same as the electrical angle, is related to the rotor mechanical angle $\delta_{m}$, (measured from a synchronously rotating frame) by

$$
\delta=\frac{p}{2} \delta_{m}
$$

where $p$ is the number of poles of the synchronous generator. Figure 2.1 shows a schematic of a synchronous generator with two poles. Also, the synchronous speed $\omega_{m R}$ used in the previous equations is actually the mechanical synchronous speed or the mechanical angular velocity at the synchronous reference value. It is related to the electrical synchronous speed $\omega_{R}$ by

$$
\omega_{R}=\frac{p}{2} \omega_{m R}
$$

By taking the derivative of Equation (2.19) on both sides, we get

$$
\dot{\delta}=\frac{p}{2} \dot{\delta}_{m}
$$


Adding Equation (2.20) and Equation (2.21) we get

$$
\dot{\delta}+\omega_{R}=\frac{p}{2} \dot{\delta}_{m}+\frac{p}{2} \omega_{m R}
$$

Thus, the electrical angular velocity $\omega$ is related to the mechanical angular velocity $\omega_{m}$ by

$$
\omega=\dot{\delta}+\omega_{R}=\frac{p}{2}\left(\dot{\delta}_{m}+\omega_{m R}\right)=\frac{p}{2} \omega_{m}
$$

Combining Equation (2.18) and Equation (2.19) we get

$$
\frac{2 M}{p} \ddot{\delta}=\frac{2 M}{p} \dot{\omega}=P_{m}-P_{e}-P_{d}
$$

Thus, we can rewrite Equation (2.24) as follows:

$$
\ddot{\delta}=\dot{\omega}=-\frac{p}{2 M} P_{d}+\frac{p}{2 M}\left(P_{m}-P_{e}\right)
$$

Since power system analysis is done in p.u. system, the swing equation is usually expressed in per unit. Dividing Equation (2.24) by the base power $S_{B}$, and substituting for $M$ results in

$$
\frac{2}{p} \frac{2 W_{k}}{\omega_{m R} S_{B}} \ddot{\delta}=\frac{2}{p} \frac{2 W_{k}}{\omega_{m R} S_{B}} \dot{\omega}=\frac{P_{m}}{S_{B}}-\frac{P_{e}}{S_{B}}-\frac{P_{d}}{S_{B}}
$$

We now define an important quantity known as the p.u. inertia constant $H[8]$.

$$
H=\frac{W_{k}}{S_{B}}
$$

The unit of $H$ is seconds. The value of $H$ ranges from 1 to 10 seconds, depending on the size and type of machine. The per unit accelerating power is related to the per unit accelerating torque by $P_{a(\text { p.u. })}=$ $T_{a(\text { p.u. })} \frac{\omega}{\omega_{R}}$. Recognizing that the electrical angular speed $\omega$ is nearly constant, and equal to $\omega_{R}$, we have the p.u. accelerating power $P_{a}$ to be numerically nearly equal to the p.u. accelerating torque $T_{a}$, i.e. $P_{a(\text { p.u. })} \cong$ $T_{a(\text { p.u. })}$. Substituting for $H$, and $P_{a(\text { p.u. })} \cong T_{a(\text { p.u.) }}$ in Equation (2.26), we get

$$
\frac{2}{p} \frac{2 H}{\omega_{m R}} \ddot{\delta}=\frac{2}{p} \frac{2 H}{\omega_{m R}} \dot{\omega}=P_{a(\text { p.u. })}=P_{m(\text { p.u. })}-P_{e(\text { p.u. })}-P_{d(\text { p.u. })} \cong T_{a(\text { p.u. })}=T_{m(\text { p.u. })}-T_{e(\text { p.u. })}-T_{d(\text { p.u. })}
$$

where $P_{m \text { (p.u.) }}, P_{e(\text { p.u.) }}$, and $P_{d \text { (p.u.) }}$ are the per unit mechanical power, electrical power, and damping power respectively. Substituting $\omega_{R}=\frac{p}{2} \omega_{m R}$ in Equation (2.28) we get

$$
\frac{2 H}{\omega_{R}} \ddot{\delta}=\frac{2 H}{\omega_{R}} \dot{\omega}=P_{m(\text { p.u. })}-P_{e(\text { p.u. })}-P_{d(\text { p.u. })} \cong T_{m(\text { p.u. })}-T_{e(\text { p.u. })}-T_{d(\text { p.u. })}
$$

In Equation (2.29), while the torque is normalized, the angular speed $\omega$ and the time $t$ are not in per unit. Thus the equation is not completely in per unit. We know that the angular speed $\omega$ and time $t$ in per unit are given by

$$
\begin{aligned}
\omega_{(p . u .)} & =\frac{\omega}{\omega_{R}} \\
t_{(\text {p.u. })} & =\omega_{R} t
\end{aligned}
$$

where the base angular velocity $\omega_{B}=\omega_{R}$. Substituting Equation (2.30) in Equation (2.29) the normalized swing equation can be written as

$$
\tau_{j} \frac{d \omega_{(\text {p.u. })}}{d t_{(\text {p.u. })}}=T_{m(\text { p.u. })}-T_{e(\text { p.u. })}-T_{d(\text { p.u. })}
$$


where $\tau_{j}=2 H \omega_{R}$. The damping torque is calculated as $T_{d(\text { p.u. })}=D \omega$, where $D$ is the damping constant. The electrical torque $T_{e \phi}$ is calculated as

$$
T_{e \phi}=i_{q} \lambda_{d}-i_{d} \lambda_{q}
$$

Also $T_{e}=\frac{T_{e \phi}}{3}$, where $T_{e}$ is the per unit electromagnetic torque defined on a three phase $(3 \phi)$ VA base, and $T_{e \phi}$ is the per unit electromagnetic torque defined on a per phase VA base. Substituting Equation (2.6) and Equation (2.7) into Equation (2.32) and writing $T_{e}$ in the p.u. system, we obtain

$$
T_{e \phi(\text { p.u. })}=3 T_{e(p . u .)}=L_{d} i_{d} i_{q}+k M_{F} i_{F} i_{q}+k M_{D} i_{D} i_{q}-L_{q} i_{d} i_{q}-k M_{Q} i_{d} i_{Q}
$$

From Equation (2.23) we have $\omega=\dot{\delta}+\omega_{R}$. If we choose $\omega_{R}$ as the frequency base and divide both sides of this equation by $\omega_{R}$ we have

$$
\frac{\omega}{\omega_{R}}=\frac{\dot{\delta}}{\omega_{R}}+\frac{\omega_{R}}{\omega_{R}}
$$

Since, $\omega_{(\text {p.u. })}=\frac{\omega}{\omega_{R}}$ and $\dot{\delta}_{(\text {p.u. })}=\frac{\dot{\delta}}{\omega_{R}}$ we can write Equation $(2.34)$ as $\dot{\delta}_{(p u)}=\omega_{(p u)}-1$. Thus, from Equation (2.29), Equation (2.33), and Equation (2.34) we can write the mechanical dynamics in the p.u. system as

$$
\begin{aligned}
\dot{\omega} & =-\frac{1}{\tau_{j}} \frac{\left(L_{d} i_{d} i_{q}+k M_{F} i_{F} i_{q}+k M_{D} i_{D} i_{q}-L_{q} i_{d} i_{q}-k M_{Q} i_{d} i_{Q}\right)}{3}-\frac{1}{\tau_{j}} D \omega+\frac{1}{\tau_{j}} T_{m} \\
\dot{\delta} & =\omega-1
\end{aligned}
$$

\subsection{Truth Model of the Synchronous Generator}

By combining the electrical dynamics and mechanical dynamics, we obtain the truth model of the synchronous generator which is highly nonlinear. Let us define

$$
L=\left[\begin{array}{ccccc}
L_{d}+L_{e} & k M_{F} & k M_{D} & 0 & 0 \\
-k M_{F} & -L_{F} & -M_{R} & 0 & 0 \\
-k M_{D} & -M_{R} & -L_{D} & 0 & 0 \\
0 & 0 & 0 & L_{q}+L_{e} & k M_{Q} \\
0 & 0 & 0 & -k M_{Q} & -L_{Q}
\end{array}\right]
$$

Also denote $\mu=\left(L_{d}+L_{e}\right) M_{R}^{2}-L_{D} L_{F}\left(L_{d}+L_{e}\right)+k^{2}\left(L_{D} M_{F}^{2}+L_{F} M_{D}^{2}-2 M_{D} M_{F} M_{R}\right)$ and $\nu=-k^{2} M_{Q}^{2}+$ $L_{Q}\left(L_{e}+L_{q}\right)$, we can derive the inverse matrix of $L$ as

$$
\begin{aligned}
& L^{-1}=\left[\begin{array}{ccccc}
\frac{1}{\mu}\left(M_{R}^{2}-L_{D} L_{F}\right) & \frac{k}{\mu}\left(M_{D} M_{R}-L_{D} M_{F}\right) & \frac{k}{\mu}\left(M_{F} M_{R}-L_{F} M_{D}\right) & 0 & 0 \\
-\frac{k}{\mu}\left(M_{D} M_{R}-L_{D} M_{F}\right) & -\frac{1}{\mu}\left(M_{D}^{2} k^{2}-L_{D}\left(L_{d}+L_{e}\right)\right) & -\frac{1}{\mu}\left(\left(L_{d}+L_{e}\right) M_{R}-M_{D} M_{F} k^{2}\right) & 0 & 0 \\
-\frac{k}{\mu}\left(M_{F} M_{R}-L_{F} M_{D}\right) & -\frac{1}{\mu}\left(\left(L_{d}+L_{e}\right) M_{R}-M_{D} M_{F} k^{2}\right) & -\frac{1}{\mu}\left(M_{F}^{2} k^{2}-L_{F}\left(L_{d}+L_{e}\right)\right) & 0 & 0 \\
0 & 0 & 0 & \frac{L_{Q}}{\nu} & \frac{k M_{Q}}{\nu} \\
0 & 0 & 0 & -\frac{k M_{Q}}{\nu} & -\frac{L_{e}+L_{q}}{\nu}
\end{array}\right] \\
& =\left[\begin{array}{ccccc}
L_{d 1} & k M_{F 1} & k M_{D 1} & 0 & 0 \\
-k M_{F 1} & -L_{F 1} & -M_{R 1} & 0 & 0 \\
-k M_{D 1} & -M_{R 1} & -L_{D 1} & 0 & 0 \\
0 & 0 & 0 & L_{q 1} & k M_{Q 1} \\
0 & 0 & 0 & -k M_{Q 1} & -L_{Q 1}
\end{array}\right]
\end{aligned}
$$

where $L_{d 1}=\frac{1}{\mu}\left(M_{R}^{2}-L_{D} L_{F}\right), L_{F 1}=\frac{1}{\mu}\left(M_{D}^{2} k^{2}-L_{D}\left(L_{d}+L_{e}\right)\right), L_{D 1}=\frac{1}{\mu}\left(M_{F}^{2} k^{2}-L_{F}\left(L_{d}+L_{e}\right)\right), M_{F 1}=$ $\frac{1}{\mu}\left(M_{D} M_{R}-L_{D} M_{F}\right), M_{D 1}=\frac{1}{\mu}\left(M_{F} M_{R}-L_{F} M_{D}\right), M_{R 1}=\frac{1}{\mu}\left(\left(L_{d}+L_{e}\right) M_{R}-M_{D} M_{F} k^{2}\right), L_{q 1}=\frac{L_{Q}}{\nu}, L_{Q 1}=$ 
$\frac{L_{e}+L_{q}}{\nu}$, and $M_{Q 1}=\frac{M_{Q}}{\nu}$. Using Equation (2.36) and Equation (2.13) we can write

$$
\begin{aligned}
\dot{i}_{d}= & -L_{d 1}\left(r+R_{e}\right) i_{d}+k M_{F 1} r_{F} i_{F}+k M_{D 1} r_{D} i_{D}-\left(L_{q}+L_{e}\right) L_{d 1} i_{q} \omega-k M_{Q} L_{d 1} i_{Q} \omega \\
& +\sqrt{3} V_{\infty} L_{d 1} \sin (\delta-\alpha)-k M_{F 1} v_{F} \\
\dot{i}_{F}= & k M_{F 1}\left(r+R_{e}\right) i_{d}-L_{F 1} r_{F} i_{F}-M_{R 1} r_{D} i_{D}+k M_{F 1}\left(L_{q}+L_{e}\right) i_{q} \omega+k^{2} M_{F 1} M_{Q} i_{Q} \omega \\
& -\sqrt{3} V_{\infty} k M_{F 1} \sin (\delta-\alpha)+L_{F 1} v_{F} \\
\dot{i}_{D}= & k M_{D 1}\left(r+R_{e}\right) i_{d}-M_{R 1} r_{F} i_{F}-L_{D 1} r_{D} i_{D}+k M_{D 1}\left(L_{q}+L_{e}\right) i_{q} \omega+k^{2} M_{D 1} M_{Q} i_{Q} \omega \\
& -\sqrt{3} V_{\infty} k M_{D 1} \sin (\delta-\alpha)+M_{R 1} v_{F} \\
\dot{i}_{q}= & L_{q 1}\left(L_{d}+L_{e}\right) i_{d} \omega+k M_{F} L_{q 1} i_{F} \omega+k M_{D} L_{q 1} i_{D} \omega-L_{q 1}\left(r+R_{e}\right) i_{q}+k M_{Q 1} r_{Q} i_{Q} \\
& -\sqrt{3} V_{\infty} L_{q 1} \cos (\delta-\alpha) \\
\dot{i}_{Q}= & -k M_{Q 1}\left(L_{d}+L_{e}\right) i_{d} \omega-k^{2} M_{Q 1} M_{F} i_{F} \omega-k^{2} M_{Q 1} M_{D} i_{D} \omega+k M_{Q 1}\left(r+R_{e}\right) i_{q}-L_{Q 1} r_{Q} i_{Q} \\
& +\sqrt{3} V_{\infty} k M_{Q 1} \cos (\delta-\alpha)
\end{aligned}
$$

Dividing both LHS and RHS of Equation (2.37) by $\sqrt{3}$ we get

$$
\begin{aligned}
\frac{\dot{i}_{d}}{\sqrt{3}}= & -L_{d 1}\left(r+R_{e}\right) \frac{i_{d}}{\sqrt{3}}+k M_{F 1} r_{F} \frac{i_{F}}{\sqrt{3}}+k M_{D 1} r_{D} \frac{i_{D}}{\sqrt{3}}-\left(L_{q}+L_{e}\right) L_{d 1} \frac{i_{q}}{\sqrt{3}} \omega-k M_{Q} L_{d 1} \frac{i_{Q}}{\sqrt{3}} \omega \\
& +V_{\infty} L_{d 1} \sin (\delta-\alpha)-k M_{F 1} \frac{v_{F}}{\sqrt{3}} \\
\frac{\dot{i}_{F}}{\sqrt{3}}= & k M_{F 1}\left(r+R_{e}\right) \frac{i_{d}}{\sqrt{3}}-L_{F 1} r_{F} \frac{i_{F}}{\sqrt{3}}-M_{R 1} r_{D} \frac{i_{D}}{\sqrt{3}}+k M_{F 1}\left(L_{q}+L_{e}\right) \frac{i_{q}}{\sqrt{3}} \omega+k^{2} M_{F 1} M_{Q} \frac{i_{Q}}{\sqrt{3}} \omega \\
& V_{\infty} k M_{F 1} \sin (\delta-\alpha)+L_{F 1} \frac{v_{F}}{\sqrt{3}} \\
\frac{\dot{i}_{D}}{\sqrt{3}=} & k M_{D 1}\left(r+R_{e}\right) \frac{i_{d}}{\sqrt{3}}-M_{R 1} r_{F} \frac{i_{F}}{\sqrt{3}}-L_{D 1} r_{D} \frac{i_{D}}{\sqrt{3}}+k M_{D 1}\left(L_{q}+L_{e}\right) \frac{i_{q}}{\sqrt{3}} \omega+k^{2} M_{D 1} M_{Q} \frac{i_{Q}}{\sqrt{3}} \omega \\
& -V_{\infty} k M_{D 1} \sin (\delta-\alpha)+M_{R 1} \frac{v_{F}}{\sqrt{3}} \\
\frac{\dot{i}_{q}}{\sqrt{3}=} & L_{q 1}\left(L_{d}+L_{e}\right) \frac{i_{d}}{\sqrt{3}} \omega+k M_{F} L_{q 1} \frac{i_{F}}{\sqrt{3}} \omega+k M_{D} L_{q 1} \frac{i_{D}}{\sqrt{3}} \omega-L_{q 1}\left(r+R_{e}\right) \frac{i_{q}}{\sqrt{3}}+k M_{Q 1} r_{Q} \frac{i_{Q}}{\sqrt{3}} \\
& -V_{\infty} L_{q 1} \cos (\delta-\alpha) \\
\frac{\dot{i}_{Q}}{\sqrt{3}=} & -k M_{Q 1}\left(L_{d}+L_{e}\right) \frac{i_{d}}{\sqrt{3}} \omega-k^{2} M_{Q 1} M_{F} \frac{i_{F}}{\sqrt{3}} \omega-k^{2} M_{Q 1} M_{D} \frac{i_{D}}{\sqrt{3}} \omega+k M_{Q 1}\left(r+R_{e}\right) \frac{i_{q}}{\sqrt{3}}-L_{Q 1} r_{Q} \frac{i_{Q}}{\sqrt{3}} \\
& +V_{\infty} k M_{Q 1} \cos (\delta-\alpha)
\end{aligned}
$$

Converting the state variables $i_{d}, i_{F}, i_{D}, i_{q}, i_{Q}$, and control input $v_{F}$ to their corresponding RMS quantities $I_{d}, I_{F}, I_{D}, I_{q}, I_{Q}$, and $V_{F}$ by substituting $\frac{i_{d}}{\sqrt{3}}=I_{d}, \frac{i_{F}}{\sqrt{3}}=I_{F}, \frac{i_{D}}{\sqrt{3}}=I_{D}, \frac{i_{q}}{\sqrt{3}}=I_{q}, \frac{i_{Q}}{\sqrt{3}}=I_{Q}$, and $\frac{v_{F}}{\sqrt{3}}=V_{F}$ in 
Equation (2.38) we get

$$
\begin{aligned}
\dot{I}_{d}= & -L_{d 1}\left(r+R_{e}\right) I_{d}+k M_{F 1} r_{F} I_{F}+k M_{D 1} r_{D} I_{D}-\left(L_{q}+L_{e}\right) L_{d 1} I_{q} \omega-k M_{Q} L_{d 1} I_{Q} \omega+V_{\infty} L_{d 1} \sin (\delta-\alpha) \\
& -k M_{F 1} V_{F} \\
\dot{I}_{F}= & k M_{F 1}\left(r+R_{e}\right) I_{d}-L_{F 1} r_{F} I_{F}-M_{R 1} r_{D} I_{D}+k M_{F 1}\left(L_{q}+L_{e}\right) I_{q} \omega+k^{2} M_{F 1} M_{Q} I_{Q} \omega-V_{\infty} k M_{F 1} \sin (\delta-\alpha) \\
& +L_{F 1} V_{F} \\
\dot{I}_{D}= & k M_{D 1}\left(r+R_{e}\right) I_{d}-M_{R 1} r_{F} I_{F}-L_{D 1} r_{D} I_{D}+k M_{D 1}\left(L_{q}+L_{e}\right) I_{q} \omega+k^{2} M_{D 1} M_{Q} I_{Q} \omega-V_{\infty} k M_{D 1} \sin (\delta-\alpha) \\
& +M_{R 1} V_{F} \\
\dot{I}_{q}= & L_{q 1}\left(L_{d}+L_{e}\right) I_{d} \omega+k M_{F} L_{q 1} I_{F} \omega+k M_{D} L_{q 1} I_{D} \omega-L_{q 1}\left(r+R_{e}\right) I_{q}+k M_{Q 1} r_{Q} I_{Q}-V_{\infty} L_{q 1} \cos (\delta-\alpha) \\
\dot{I}_{Q}= & -k M_{Q 1}\left(L_{d}+L_{e}\right) I_{d} \omega-k^{2} M_{Q 1} M_{F} I_{F} \omega-k^{2} M_{Q 1} M_{D} I_{D} \omega+k M_{Q 1}\left(r+R_{e}\right) I_{q}-L_{Q 1} r_{Q} I_{Q} \\
& +V_{\infty} k M_{Q 1} \cos (\delta-\alpha) \\
&
\end{aligned}
$$

Equation (2.35) can be written as

$$
\begin{aligned}
& \dot{\omega}=-\frac{1}{\tau_{j}}\left(L_{d} \frac{i_{d}}{\sqrt{3}} \frac{i_{q}}{\sqrt{3}}+k M_{F} \frac{i_{F}}{\sqrt{3}} \frac{i_{q}}{\sqrt{3}}+k M_{D} \frac{i_{D}}{\sqrt{3}} \frac{i_{q}}{\sqrt{3}}-L_{q} \frac{i_{d}}{\sqrt{3}} \frac{i_{q}}{\sqrt{3}}-k M_{Q} \frac{i_{d}}{\sqrt{3}} \frac{i_{Q}}{\sqrt{3}}\right)-\frac{1}{\tau_{j}} D \omega+\frac{1}{\tau_{j}} T_{m} \\
& \dot{\delta}=\omega-1
\end{aligned}
$$

Substituting $\frac{i_{d}}{\sqrt{3}}=I_{d}, \frac{i_{F}}{\sqrt{3}}=I_{F}, \frac{i_{D}}{\sqrt{3}}=I_{D}, \frac{i_{q}}{\sqrt{3}}=I_{q}, \frac{i_{Q}}{\sqrt{3}}=I_{Q}$ in Equation $(2.40)$

$$
\begin{aligned}
& \dot{\omega}=-\frac{1}{\tau_{j}}\left(\left(L_{d}-L_{q}\right) I_{d} I_{q}+k M_{F} I_{F} I_{q}+k M_{D} I_{D} I_{q}-k M_{Q} I_{d} I_{Q}\right)-\frac{1}{\tau_{j}} D \omega+\frac{1}{\tau_{j}} T_{m} \\
& \dot{\delta}=\omega-1
\end{aligned}
$$

Equation (2.39) and Equation (2.41) can be combined to get the truth model of the synchronous generator

$$
\begin{aligned}
\dot{I}_{d}= & L_{d 1}\left(r+R_{e}\right) I_{d}+k M_{F 1} r_{F} I_{F}+k M_{D 1} r_{D} I_{D}-\left(L_{q}+L_{e}\right) L_{d 1} I_{q} \omega-k M_{Q} L_{d 1} I_{Q} \omega+V_{\infty} L_{d 1} \sin (\delta-\alpha) \\
& -k M_{F 1} V_{F} \\
\dot{I}_{F}= & k M_{F 1}\left(r+R_{e}\right) I_{d}-L_{F 1} r_{F} I_{F}-M_{R 1} r_{D} I_{D}+k M_{F 1}\left(L_{q}+L_{e}\right) I_{q} \omega+k^{2} M_{F 1} M_{Q} I_{Q} \omega-V_{\infty} k M_{F 1} \sin (\delta-\alpha) \\
& +L_{F 1} V_{F} \\
\dot{I}_{D}= & k M_{D 1}\left(r+R_{e}\right) I_{d}-M_{R 1} r_{F} I_{F}-L_{D 1} r_{D} I_{D}+k M_{D 1}\left(L_{q}+L_{e}\right) I_{q} \omega+k^{2} M_{D 1} M_{Q} I_{Q} \omega-V_{\infty} k M_{D 1} \sin (\delta-\alpha) \\
& +M_{R 1} V_{F} \\
\dot{I}_{q}= & L_{q 1}\left(L_{d}+L_{e}\right) I_{d} \omega+k M_{F} L_{q 1} I_{F} \omega+k M_{D} L_{q 1} I_{D} \omega-L_{q 1}\left(r+R_{e}\right) I_{q}+k M_{Q 1} r_{Q} I_{Q}-V_{\infty} L_{q 1} \cos (\delta-\alpha) \\
\dot{I}_{Q}= & -k M_{Q 1}\left(L_{d}+L_{e}\right) I_{d} \omega-k^{2} M_{Q 1} M_{F} I_{F} \omega-k^{2} M_{Q 1} M_{D} I_{D} \omega+k M_{Q 1}\left(r+R_{e}\right) I_{q}-L_{Q 1} r_{Q} I_{Q} \\
& +V_{\infty} k M_{Q 1} \cos (\delta-\alpha) \\
\dot{\omega}=- & \frac{1}{\tau_{j}}\left(\left(L_{d}-L_{q}\right) I_{d} I_{q}+k M_{F} I_{F} I_{q}+k M_{D} I_{D} I_{q}-k M_{Q} I_{d} I_{Q}\right)-\frac{1}{\tau_{j}} D \omega+\frac{1}{\tau_{j}} T_{m} \\
\dot{\delta}=\omega-1 &
\end{aligned}
$$


For simplification of the above expression let us denote:

$$
\begin{aligned}
& F_{11}=-L_{d 1}\left(r+R_{e}\right), \quad F_{12}=k M_{F 1} r_{F}, \quad F_{13}=k M_{D 1} r_{D}, \quad F_{14}=-\left(L_{q}+L_{e}\right) L_{d 1}, \quad F_{15}=-k M_{Q} L_{d 1}, \\
& F_{16}=V_{\infty} L_{d 1}, \quad G_{11}=-k M_{F 1}, \quad F_{21}=k M_{F 1}\left(r+R_{e}\right), \quad F_{22}=-L_{F 1} r_{F}, \quad F_{23}=-M_{R 1} r_{D}, \\
& F_{24}=k M_{F 1}\left(L_{q}+L_{e}\right), \quad F_{25}=k^{2} M_{F 1} M_{Q}, \quad F_{26}=-V_{\infty} k M_{F 1}, \quad G_{21}=L_{F 1}, \quad F_{31}=k M_{D 1}\left(r+R_{e}\right), \\
& F_{32}=-M_{R 1} r_{F}, \quad F_{33}=-L_{D 1} r_{D}, \quad F_{34}=k M_{D 1}\left(L_{q}+L_{e}\right), \quad F_{35}=k^{2} M_{D 1} M_{Q}, \quad F_{36}=-V_{\infty} k M_{D 1}, \\
& G_{31}=M_{R 1}, \quad F_{41}=L_{q 1}\left(L_{d}+L_{e}\right), \quad F_{42}=k M_{F} L_{q 1}, \quad F_{43}=k M_{D} L_{q 1}, \quad F_{44}=-L_{q 1}\left(r+R_{e}\right), \\
& F_{45}=k M_{Q 1} r_{Q}, \quad F_{46}=-V_{\infty} L_{q 1}, \quad F_{51}=-k M_{Q 1}\left(L_{d}+L_{e}\right), \quad F_{52}=-k^{2} M_{Q 1} M_{F}, \quad F_{53}=-k^{2} M_{Q 1} M_{D}, \\
& F_{54}=k M_{Q 1}\left(r+R_{e}\right), \quad F_{55}=-L_{Q 1} r_{Q}, \quad F_{56}=V_{\infty} k M_{Q 1}, \quad F_{61}=-\frac{1}{\tau_{j}}\left(L_{d}-L_{q}\right), \\
& F_{62}=-\frac{1}{\tau_{j}} k M_{F}, \quad F_{63}=-\frac{1}{\tau_{j}} k M_{D}, \quad F_{64}=\frac{1}{\tau_{j}} k M_{Q}, \quad F_{65}=-\frac{1}{\tau_{j}} D .
\end{aligned}
$$

Thus, the $7^{\text {th }}$ order truth model of the synchronous generator connected to an infinite bus in per unit can be written in the nonlinear state-space form

$$
\begin{aligned}
& \dot{I}_{d}=F_{11} I_{d}+F_{12} I_{F}+F_{13} I_{D}+F_{14} I_{q} \omega+F_{15} I_{Q} \omega+F_{16} \sin (\delta-\alpha)+G_{11} V_{F} \\
& \dot{I}_{F}=F_{21} I_{d}+F_{22} I_{F}+F_{23} I_{D}+F_{24} I_{q} \omega+F_{25} I_{Q} \omega+F_{26} \sin (\delta-\alpha)+G_{21} V_{F} \\
& \dot{I}_{D}=F_{31} I_{d}+F_{32} I_{F}+F_{33} I_{D}+F_{34} I_{q} \omega+F_{35} I_{Q} \omega+F_{36} \sin (\delta-\alpha)+G_{31} V_{F} \\
& \dot{I}_{q}=F_{41} I_{d} \omega+F_{42} I_{F} \omega+F_{43} I_{D} \omega+F_{44} I_{q}+F_{45} I_{Q}+F_{46} \cos (\delta-\alpha) \\
& \dot{I}_{Q}=F_{51} I_{d} \omega+F_{52} I_{F} \omega+F_{53} I_{D} \omega+F_{54} I_{q}+F_{55} I_{Q}+F_{56} \cos (\delta-\alpha) \\
& \dot{\omega}=F_{61} I_{d} I_{q}+F_{62} I_{F} I_{q}+F_{63} I_{D} I_{q}+F_{64} I_{d} I_{Q}+F_{65} \omega+F_{66} T_{m} \\
& \dot{\delta}=\omega-1
\end{aligned}
$$

\subsection{Model of the Turbine-Governor System}

In this section, we present the dynamics of the turbine-governor system. For the sake of simplicity we assume a linear model of the turbine-governor system [9].

- Turbine dynamics: The dynamics of the turbine are modeled by

$$
\dot{P}_{m}=-\frac{1}{\tau_{T}} P_{m}+\frac{K_{T}}{\tau_{T}} G_{V}
$$

where $P_{m}$ is the mechanical power output of the turbine, $G_{V}$ is the gate opening of the turbine, $\tau_{T}$ is the time constant of the turbine, and $K_{T}$ is the gain of the turbine. As done in Equation (2.28) we have the per unit mechanical power numerically equal to the per unit mechanical torque, i.e. $P_{m(p . u .)}=T_{m(p . u .)}$. Therefore, the per unit turbine dynamics are

$$
\dot{T}_{m(p . u .)}=-\frac{1}{\tau_{T}} T_{m(p . u .)}+\frac{K_{T}}{\tau_{T}} G_{V(p . u .)}
$$

- Governor dynamics: The dynamics of the governor in per unit are

$$
\dot{G}_{V(\text { p.u. })}=-\frac{1}{\tau_{G}} G_{V(\text { p.u. })}+\frac{K_{G}}{\tau_{G}}\left(u_{T}-\frac{\omega_{(p . u .)}}{R_{T}}\right)
$$

where $u_{T}$ is the turbine valve control, $\tau_{G}$ is the time constant of the speed governor, $K_{G}$ is the gain of the speed governor, and $R_{T}$ is the regulation constant in per unit.

Parameters of the turbine-governor system are

$$
K_{T}=1, \quad K_{G}=1, \quad \tau_{T}=0.5, \quad \tau_{G}=0.2, \quad R_{T}=20
$$




\subsection{Truth model of the combined Synchronous Generator and Turbine-Governor Sys- tem connected to an infinite bus}

In this section, we present the truth model of the combined synchronous generator and turbine-governor system connected to an infinite bus. This model consists of 7 nonlinear differential equations of the synchronous generator and 2 linear differential equations of the turbine-governor system. Thus, the combined system consists of 9 differential equations. Combining Equation (2.44), Equation (2.46), and Equation (2.47) the truth model of the combined synchronous generator and turbine-governor system connected to an infinite bus, can be written as

$$
\begin{aligned}
& \dot{I}_{d}=F_{11} I_{d}+F_{12} I_{F}+F_{13} I_{D}+F_{14} I_{q} \omega+F_{15} I_{Q} \omega+F_{16} \sin (\delta-\alpha)+G_{11} V_{F} \\
& \dot{I}_{F}=F_{21} I_{d}+F_{22} I_{F}+F_{23} I_{D}+F_{24} I_{q} \omega+F_{25} I_{Q} \omega+F_{26} \sin (\delta-\alpha)+G_{21} V_{F} \\
& \dot{I}_{D}=F_{31} I_{d}+F_{32} I_{F}+F_{33} I_{D}+F_{34} I_{q} \omega+F_{35} I_{Q} \omega+F_{36} \sin (\delta-\alpha)+G_{31} V_{F} \\
& \dot{I}_{q}=F_{41} I_{d} \omega+F_{42} I_{F} \omega+F_{43} I_{D} \omega+F_{44} I_{q}+F_{45} I_{Q}+F_{46} \cos (\delta-\alpha) \\
& \dot{I}_{Q}=F_{51} I_{d} \omega+F_{52} I_{F} \omega+F_{53} I_{D} \omega+F_{54} I_{q}+F_{55} I_{Q}+F_{56} \cos (\delta-\alpha) \\
& \dot{\omega}=F_{61} I_{d} I_{q}+F_{62} I_{F} I_{q}+F_{63} I_{D} I_{q}+F_{64} I_{d} I_{Q}+F_{65} \omega+F_{66} T_{m} \\
& \dot{\delta}=\omega-1 \\
& \dot{T}_{m}=F_{81} T_{m}+F_{82} G_{V} \\
& \dot{G}_{V}=F_{91} \omega+F_{92} G_{V}+G_{92} u_{T}
\end{aligned}
$$

where $F_{81}=-\frac{1}{\tau_{T}}, F_{82}=\frac{K_{T}}{\tau_{T}}, F_{91}=-\frac{K_{G}}{\tau_{G} R_{T}}, F_{92}=-\frac{1}{\tau_{G}}, G_{92}=\frac{K_{G}}{\tau_{G}}$. Let $x=\left[I_{d}, I_{F}, I_{D}, I_{q}, I_{Q}, \omega, \delta, T_{m}, G_{V}\right]^{\mathrm{T}}$ be the vector of state variables, $u=\left[V_{F}, u_{T}\right]^{\mathrm{T}}$ the vector of control inputs, and $y=\left[V_{t}, \omega\right]^{\mathrm{T}}$ the vector of outputs, then Equation (2.49) can be written in the usual state-space form

$$
\begin{aligned}
& \dot{x}=F(x)+G(x) u \\
& y=h(x)
\end{aligned}
$$

where

$$
\begin{array}{r}
F(x)=\left(\begin{array}{c}
F_{11} x_{1}+F_{12} x_{2}+F_{13} x_{3}+F_{14} x_{4} x_{6}+F_{15} x_{5} x_{6}+F_{16} \sin \left(x_{7}-\alpha\right) \\
F_{21} x_{1}+F_{22} x_{2}+F_{23} x_{3}+F_{24} x_{4} x_{6}+F_{25} x_{5} x_{6}+F_{26} \sin \left(x_{7}-\alpha\right) \\
F_{31} x_{1}+F_{32} x_{2}+F_{33} x_{3}+F_{34} x_{4} x_{6}+F_{35} x_{5} x_{6}+F_{36} \sin \left(x_{7}-\alpha\right) \\
F_{41} x_{1} x_{6}+F_{42} x_{2} x_{6}+F_{43} x_{3} x_{6}+F_{44} x_{4}+F_{45} x_{5}+F_{46} \cos \left(x_{7}-\alpha\right) \\
F_{51} x_{1} x_{6}+F_{52} x_{2} x_{6}+F_{53} x_{3} x_{6}+F_{54} x_{4}+F_{55} x_{5}+F_{56} \cos \left(x_{7}-\alpha\right) \\
F_{61} x_{1} x_{4}+F_{62} x_{2} x_{4}+F_{63} x_{3} x_{4}+F_{64} x_{1} x_{5}+F_{65} x_{6}+F_{66} x_{8} \\
x_{6}-1 \\
F_{81} x_{8}+F_{82} x_{9} \\
F_{91} x_{6}+F_{92} x_{9} \\
\end{array} \quad\left(\begin{array}{cc}
G_{11} & 0 \\
G_{21} & 0 \\
G_{31} & 0 \\
0 & 0 \\
0 & 0 \\
0 & 0 \\
0 & G_{92}
\end{array}\right)\right. \\
G(x)=
\end{array}
$$

\subsection{Derivation of the Output Generator Terminal Voltage for the Truth Model}

The synchronous generator and turbine-governor system connected to an infinite bus is a MIMO system with two inputs: excitation field voltage $V_{F}$ and turbine valve control $u_{T}$, i.e. $u=\left[u_{1}, u_{2}\right]^{\mathrm{T}}=\left[V_{F}, u_{T}\right]^{\mathrm{T}}$, and two regulated outputs: generator terminal voltage $V_{t}$ and rotor angle $\delta$, i.e. $y=\left[y_{1}, y_{2}\right]^{\mathrm{T}}=\left[V_{t}, \delta\right]^{\mathrm{T}}$. Since 
the rotor angle is difficult to measure in a practical system, we use the angular frequency $\omega$ instead of the rotor angle as the second output in all our work, as the frequency $\omega$ can be easily measured. In this section we derive an expression for the generator terminal voltage $V_{t}$. From Equation (2.12) we have

$$
\begin{aligned}
& v_{d}=R_{e} i_{d}+L_{e} \dot{i}_{d}+\omega L_{e} i_{q}-\sqrt{3} V_{\infty} \sin (\delta-\alpha) \\
& v_{q}=R_{e} i_{q}+L_{e} \dot{i}_{q}-\omega L_{e} i_{d}+\sqrt{3} V_{\infty} \cos (\delta-\alpha)
\end{aligned}
$$

Dividing Equation (2.52) by $\sqrt{3}$ and substituting $\frac{v_{d}}{\sqrt{3}}=V_{d}, \frac{v_{q}}{\sqrt{3}}=V_{q}, \frac{i_{d}}{\sqrt{3}}=I_{d}$, and $\frac{i_{q}}{\sqrt{3}}=I_{q}$, where $V_{d}, V_{q}$, $I_{d}$, and $I_{q}$ are the corresponding RMS values, we get

$$
\begin{aligned}
& V_{d}=R_{e} I_{d}+L_{e} \dot{I}_{d}+\omega L_{e} I_{q}-V_{\infty} \sin (\delta-\alpha) \\
& V_{q}=R_{e} I_{q}+L_{e} \dot{I}_{q}-\omega L_{e} I_{d}+V_{\infty} \cos (\delta-\alpha)
\end{aligned}
$$

Substituting $\dot{I}_{d}$ and $\dot{I}_{q}$ from Equation (2.49) in Equation (2.53)

$$
\begin{aligned}
V_{d}= & R_{e} I_{d}+L_{e}\left(F_{11} I_{d}+F_{12} I_{F}+F_{13} I_{D}+F_{14} I_{q} \omega+F_{15} I_{Q} \omega+F_{16} \sin (\delta-\alpha)+G_{11} v_{F}\right)+\omega L_{e} I_{q} \\
& -V_{\infty} \sin (\delta-\alpha) \\
V_{q}= & R_{e} I_{q}+L_{e}\left(F_{41} I_{d} \omega+F_{42} I_{F} \omega+F_{43} I_{D} \omega+F_{44} I_{q}+F_{45} I_{Q}+F_{46} \cos (\delta-\alpha)\right)-\omega L_{e} I_{d}+V_{\infty} \cos (\delta-\alpha)
\end{aligned}
$$

Simplifying and rearranging Equation (2.54) we get

$$
\begin{aligned}
V_{d}= & \left(R_{e}+L_{e} F_{11}\right) I_{d}+L_{e} F_{12} I_{F}+L_{e} F_{13} I_{D}+\left(L_{e} F_{14}+L_{e}\right) I_{q} \omega+L_{e} F_{15} I_{Q} \omega+\left(L_{e} F_{16}-V_{\infty}\right) \sin (\delta-\alpha) \\
& +L_{e} G_{11} V_{F} \\
V_{q}= & \left(L_{e} F_{41}-L_{e}\right) I_{d} \omega+L_{e} F_{42} I_{F} \omega+L_{e} F_{43} I_{D} \omega+\left(R_{e}+L_{e} F_{44}\right) I_{q}+L_{e} F_{45} I_{Q}+\left(L_{e} F_{46}+V_{\infty}\right) \cos (\delta-\alpha)
\end{aligned}
$$

For simplification of Equation (2.55) let us denote $R_{e}+L_{e} F_{11}=y_{11}, L_{e} F_{12}=y_{12}, L_{e} F_{13}=y_{13}, L_{e} F_{14}+$ $L_{e}=y_{14}, L_{e} F_{15}=y_{15}, L_{e} F_{16}-V_{\infty}=y_{16}, L_{e} G_{11}=i_{11}, L_{e} F_{41}-L_{e}=y_{21}, L_{e} F_{42}=y_{22}, L_{e} F_{43}=y_{23}$, $R_{e}+L_{e} F_{44}=y_{24}, L_{e} F_{45}=y_{25}$, and $L_{e} F_{46}+V_{\infty}=y_{26}$. Thus, Equation (2.55) can be simplified to

$$
\begin{aligned}
& V_{d}=y_{11} I_{d}+y_{12} I_{F}+y_{13} I_{D}+y_{14} I_{q} \omega+y_{15} I_{Q} \omega+y_{16} \sin (\delta-\alpha)+i_{11} V_{F} \\
& V_{q}=y_{21} I_{d} \omega+y_{22} I_{F} \omega+y_{23} I_{D} \omega+y_{24} I_{q}+y_{25} I_{Q}+y_{26} \cos (\delta-\alpha)
\end{aligned}
$$

The generator terminal voltage $V_{t}$ is computed as

$$
V_{t}=\sqrt{V_{d}^{2}+V_{q}^{2}}
$$

where $V_{d}$ and $V_{q}$ are as given in Equation (2.55). The output generator terminal voltage, $y_{1}=V_{t}$, as a function of the states $x$, and control inputs $u$, is

$$
\begin{aligned}
& V_{d}=y_{11} x_{1}+y_{12} x_{2}+y_{13} x_{3}+y_{14} x_{4} x_{6}+y_{15} x_{5} x_{6}+y_{16} \sin \left(x_{7}-\alpha\right)+i_{11} u_{1} \\
& V_{q}=y_{21} x_{1} x_{6}+y_{22} x_{2} x_{6}+y_{23} x_{3} x_{6}+y_{24} x_{4}+y_{25} x_{5}+y_{26} \cos \left(x_{7}-\alpha\right) \\
& y_{1}=V_{t}=\sqrt{V_{d}^{2}+V_{q}^{2}}
\end{aligned}
$$

Therefore, the output equation is given by

$$
y=h(x)=\left[\begin{array}{l}
V_{t} \\
x_{6}
\end{array}\right]
$$

where $V_{t}$ is as given in Equation (2.57). 


\section{The Reduced Order Simplified Model}

A detailed derivation of the reduced order simplified model of a synchronous generator connected to an infinite bus is given in the Appendix.

\subsection{Reduced order model of the combined Synchronous Generator and Turbine-Governor system connected to an infinite bus}

The final system equations for the reduced order simplified model of the synchronous generator are summarized below

$$
\begin{aligned}
\dot{E}_{q}^{\prime} & =\frac{1}{\tau_{d 0}^{\prime}}\left(E_{F D}-E_{q}^{\prime}+\left(L_{d}-L_{d}^{\prime}\right) I_{d}\right) \\
\dot{\omega} & =\frac{1}{\tau_{j}}\left[T_{m}-D \omega-\left(E_{q}^{\prime} I_{q}-\left(L_{q}-L_{d}^{\prime}\right) I_{d} I_{q}\right)\right] \\
\dot{\delta} & =\omega-1
\end{aligned}
$$

where $E_{d}^{\prime}$ is the $d$ axis voltage behind the transient reactance $L_{q}^{\prime}$, and $E_{q}^{\prime}$ is the $q$ axis voltage behind the transient reactance $L_{d}^{\prime}$, where $L_{d}^{\prime}=L_{d}-\frac{\left(k M_{F}\right)^{2}}{L_{F}} \cdot \tau_{d 0}^{\prime}$ is the $d$ axis transient open circuit time constant and is given by the relation $\tau_{d 0}^{\prime}=\frac{L_{F}}{r_{F}} . E_{F D}$ is the excitation field emf, and $\tau_{j}=2 H \omega_{R}$. Also $I_{d}$ and $I_{q}$ are the direct axis and quadrature axis currents respectively. $E_{d}^{\prime}$ is given by an algebraic constraint

$$
E_{d}^{\prime}=-\left(L_{q}-L_{q}^{\prime}\right) I_{q}
$$

Note that all the variables in the third axis model of Equation (3.1) are RMS quantities.

By applying Kirchhoff's voltage law (KVL) to the $d$ axis and $q$ axis stator circuits, the $d$ axis and $q$ axis stator voltage equations of a synchronous generator in per unit are

$$
\begin{aligned}
& V_{d}=-r I_{d}-L_{q}^{\prime} I_{q}+E_{d}^{\prime} \\
& V_{q}=-r I_{q}+L_{d}^{\prime} I_{d}+E_{q}^{\prime}
\end{aligned}
$$

On substituting $E_{d}^{\prime}$ as given in Equation (3.2) in Equation (3.3) we get

$$
\begin{aligned}
& V_{d}=-r I_{d}-L_{q} I_{q} \\
& V_{q}=-r I_{q}+L_{d}^{\prime} I_{d}+E_{q}^{\prime}
\end{aligned}
$$

By applying KVL to a synchronous generator connected to an infinite bus the stator voltage equations can be written as

$$
\begin{aligned}
V_{\infty d} & =V_{d}-R_{e} I_{d}-L_{e} I_{q} \\
& =-\left(r+R_{e}\right) I_{d}-\left(L_{q}+L_{e}\right) I_{q} \\
V_{\infty q} & =V_{q}-R_{e} I_{q}+L_{e} I_{d} \\
& =-\left(r+R_{e}\right) I_{q}+\left(L_{d}^{\prime}+L_{e}\right) I_{d}+E_{q}^{\prime}
\end{aligned}
$$

where $V_{\infty d}$ and $V_{\infty d}$ are direct axis and quadrature axis infinite bus voltages respectively, $R_{e}$ is the resistance and $L_{e}$ is the inductance of the infinite bus. We now solve the two simultaneous equations given in Equation (3.5) to determine the two unknowns $I_{d}$ and $I_{q}$. On dividing $V_{\infty d}$ in Equation (3.5) by $-\left(L_{q}+L_{e}\right)$

$$
\frac{V_{\infty d}}{-\left(L_{q}+L_{e}\right)}=\frac{-\left(r+R_{e}\right)}{-\left(L_{q}+L_{e}\right)} I_{d}+I_{q}
$$

Similarly dividing $V_{\infty q}$ in Equation (3.5) by $r+R_{e}$

$$
\frac{V_{\infty q}}{\left(r+R_{e}\right)}=-I_{q}+\frac{\left(L_{d}^{\prime}+L_{e}\right)}{\left(r+R_{e}\right)} I_{d}+\frac{E_{q}^{\prime}}{\left(r+R_{e}\right)}
$$


Now we add Equation (3.6) and Equation (3.7) and compute $I_{d}$

$$
I_{d}=\frac{-\left(E_{q}^{\prime}-V_{\infty q}\right)\left(L_{q}+L_{e}\right)-V_{\infty d}\left(r+R_{e}\right)}{\left(r+R_{e}\right)^{2}+\left(L_{d}^{\prime}+L_{e}\right)\left(L_{q}+L_{e}\right)}
$$

$I_{q}$ is determined in a similar fashion. Dividing $V_{\infty d}$ in Equation $(3.5)$ by $\left(r+R_{e}\right)$

$$
\frac{V_{\infty d}}{\left(r+R_{e}\right)}=-I_{d}-\frac{\left(L_{q}+L_{e}\right)}{\left(r+R_{e}\right)} I_{q}
$$

Also dividing $V_{\infty q}$ in Equation (3.5) by $L_{d}^{\prime}+L_{e}$

$$
\frac{V_{\infty q}}{\left(L_{d}^{\prime}+L_{e}\right)}=-\frac{\left(r+R_{e}\right)}{\left(L_{d}^{\prime}+L_{e}\right)} I_{q}+I_{d}+\frac{E_{q}^{\prime}}{\left(L_{d}^{\prime}+L_{e}\right)}
$$

Now we add Equation (3.9) and Equation (3.10) and compute $I_{q}$

$$
I_{q}=\frac{\left(E_{q}^{\prime}-V_{\infty q}\right)\left(r+R_{e}\right)-V_{\infty d}\left(L_{d}^{\prime}+L_{e}\right)}{\left(r+R_{e}\right)^{2}+\left(L_{d}^{\prime}+L_{e}\right)\left(L_{q}+L_{e}\right)}
$$

We substitute $I_{d}$ and $I_{q}$ as given in Equation (3.8) and Equation (3.11) in the reduced order simplified model of the synchronous generator as given in Equation (3.1) to get

$$
\dot{E}_{q}^{\prime}=\frac{1}{\tau_{d 0}^{\prime}}\left(E_{F D}-E_{q}^{\prime}+\left(L_{d}-L_{d}^{\prime}\right)\left(\frac{-\left(E_{q}^{\prime}-V_{\infty q}\right)\left(L_{q}+L_{e}\right)-V_{\infty d}\left(r+R_{e}\right)}{\left(r+R_{e}\right)^{2}+\left(L_{d}^{\prime}+L_{e}\right)\left(L_{q}+L_{e}\right)}\right)\right)
$$

For simplification of the above equation we make the following substitutions:

$L_{q}+L_{e}=L_{1}, r+R_{e}=R_{1}, L_{d}-L_{d}^{\prime}=L_{2}$ and $\left(r+R_{e}\right)^{2}+\left(L_{d}^{\prime}+L_{e}\right)\left(L_{q}+L_{e}\right)=M_{1}$

Thus, Equation (3.12) can be rewritten as

$$
\dot{E}_{q}^{\prime}=\frac{1}{\tau_{d 0}^{\prime}}\left(E_{F D}-E_{q}^{\prime}-\frac{L_{2} L_{1}}{M_{1}} E_{q}^{\prime}+\frac{L_{2} L_{1}}{M_{1}} V_{\infty q}-\frac{L_{2} R_{1}}{M_{1}} V_{\infty d}\right)
$$

Substituting $V_{\infty d}=-V_{\infty} \sin (\delta-\alpha)$ and $V_{\infty q}=V_{\infty} \cos (\delta-\alpha)$ in Equation (3.13) and rearranging it we get

$$
\dot{E}_{q}^{\prime}=\frac{-\left(1+\frac{L_{2} L_{1}}{M_{1}}\right)}{\tau_{d 0}^{\prime}} E_{q}^{\prime}+\frac{L_{2} L_{1} V_{\infty}}{M_{1} \tau_{d 0}^{\prime}} \cos (\delta-\alpha)+\frac{L_{2} R_{1} V_{\infty}}{M_{1} \tau_{d 0}^{\prime}} \sin (\delta-\alpha)+\frac{1}{\tau_{d 0}^{\prime}} E_{F D}
$$

Let us denote $\frac{-\left(1+\frac{L_{2} L_{1}}{M_{1}}\right)}{\tau_{d 0}^{\prime}}=f_{11}, \frac{L_{2} L_{1} V_{\infty}}{M_{1} \tau_{d 0}^{\prime}}=f_{12}, \frac{L_{2} R_{1} V_{\infty}}{M_{1} \tau_{d 0}^{\prime}}=f_{13}$ and $\frac{1}{\tau_{d 0}^{\prime}}=g_{11}$. Thus, Equation (3.14) can be rewritten as

$$
\dot{E}_{q}^{\prime}=f_{11} E_{q}^{\prime}+f_{12} \cos (\delta-\alpha)+f_{13} \sin (\delta-\alpha)+g_{11} E_{F D}
$$

Substituting $I_{d}$ and $I_{q}$ in the equation for $\dot{\omega}$ in the reduced order simplified model of the synchronous generator as given in Equation (3.1) we get

$$
\begin{aligned}
\dot{\omega}= & \frac{1}{\tau_{j}}\left[T_{m}-D \omega-\left(E_{q}^{\prime}\left(\frac{\left(E_{q}^{\prime}-V_{\infty q}\right)\left(r+R_{e}\right)-V_{\infty d}\left(L_{d}^{\prime}+L_{e}\right)}{\left(r+R_{e}\right)^{2}+\left(L_{d}^{\prime}+L_{e}\right)\left(L_{q}+L_{e}\right)}\right)\right.\right. \\
& \left.\left.-\left(L_{q}-L_{d}^{\prime}\right)\left(\frac{-\left(E_{q}^{\prime}-V_{\infty q}\right)\left(L_{q}+L_{e}\right)-V_{\infty d}\left(r+R_{e}\right)}{\left(r+R_{e}\right)^{2}+\left(L_{d}^{\prime}+L_{e}\right)\left(L_{q}+L_{e}\right)}\right)\left(\frac{\left(E_{q}^{\prime}-V_{\infty q}\right)\left(r+R_{e}\right)-V_{\infty d}\left(L_{d}^{\prime}+L_{e}\right)}{\left(r+R_{e}\right)^{2}+\left(L_{d}^{\prime}+L_{e}\right)\left(L_{q}+L_{e}\right)}\right)\right)\right]
\end{aligned}
$$


Let us denote $L_{d}^{\prime}+L_{e}=L_{3}$ and $L_{q}-L_{d}^{\prime}=L_{4}$. Substituting $V_{\infty d}=-V_{\infty} \sin (\delta-\alpha)$ and $V_{\infty q}=V_{\infty} \cos (\delta-\alpha)$ in Equation (3.16) and on simplifying we get

$$
\begin{aligned}
\dot{\omega}= & -\left(\frac{R_{1}}{M_{1} \tau_{j}}+\frac{L_{4} L_{1} R_{1}}{M_{1}^{2} \tau_{j}}\right) E_{q}^{\prime 2}+\left(\frac{R_{1}}{M_{1} \tau_{j}}+\frac{2 L_{4} L_{1} R_{1}}{M_{1}^{2} \tau_{j}}\right) V_{\infty} E_{q}^{\prime} \cos (\delta-\alpha) \\
& -\left(\frac{L_{3}}{M_{1} \tau_{j}}+\frac{L_{4} L_{1} L_{3}}{M_{1}^{2} \tau_{j}}-\frac{L_{4} R_{1}^{2}}{M_{1}^{2} \tau_{j}}\right) V_{\infty} E_{q}^{\prime} \sin (\delta-\alpha) \\
& -\left(\frac{L_{4} R_{1}^{2}}{M_{1}^{2} \tau_{j}}-\frac{L_{4} L_{1} L_{3}}{M_{1}^{2} \tau_{j}}\right) V_{\infty}^{2} \sin (\delta-\alpha) \cos (\delta-\alpha)-\left(\frac{L_{4} L_{1} R_{1} V_{\infty}^{2}}{M_{1}^{2} \tau_{j}}\right) \cos ^{2}(\delta-\alpha) \\
& +\left(\frac{L_{4} L_{3} R_{1} V_{\infty}^{2}}{M_{1}^{2} \tau_{j}}\right) \sin ^{2}(\delta-\alpha)-\frac{D}{\tau_{j}} \omega+\frac{1}{\tau_{j}} T_{m}
\end{aligned}
$$

Let us denote

$$
\begin{aligned}
& -\left(\frac{R_{1}}{M_{1} \tau_{j}}+\frac{L_{4} L_{1} R_{1}}{M_{1}^{2} \tau_{j}}\right)=f_{21},\left(\frac{R_{1}}{M_{1} \tau_{j}}+\frac{2 L_{4} L_{1} R_{1}}{M_{1}^{2} \tau_{j}}\right) V_{\infty}=f_{22}, \\
& -\left(\frac{L_{3}}{M_{1} \tau_{j}}+\frac{L_{4} L_{1} L_{3}}{M_{1}^{2} \tau_{j}}-\frac{L_{4} R_{1}^{2}}{M_{1}^{2} \tau_{j}}\right) V_{\infty}=f_{23},-\left(\frac{L_{4} R_{1}^{2}}{M_{1}^{2} \tau_{j}}-\frac{L_{4} L_{1} L_{3}}{M_{1}^{2} \tau_{j}}\right) V_{\infty}^{2}=f_{24}, \\
& -\left(\frac{L_{4} L_{1} R_{1} V_{\infty}^{2}}{M_{1}^{2} \tau_{j}}\right)=f_{25},\left(\frac{L_{4} L_{3} R_{1} V_{\infty}^{2}}{M_{1}^{2} \tau_{j}}\right)=f_{26},-\frac{D}{\tau_{j}}=f_{27}, \text { and } \frac{1}{\tau_{j}}=f_{28}
\end{aligned}
$$

Thus, Equation (3.17) can be rewritten as

$$
\begin{aligned}
\dot{\omega}= & f_{21} E_{q}^{\prime 2}+f_{22} E_{q}^{\prime} \cos (\delta-\alpha)+f_{23} E_{q}^{\prime} \sin (\delta-\alpha)+f_{24} \sin (\delta-\alpha) \cos (\delta-\alpha) \\
& +f_{25} \cos ^{2}(\delta-\alpha)+f_{26} \sin ^{2}(\delta-\alpha)+f_{27} \omega+f_{28} T_{m}
\end{aligned}
$$

We can express the reduced order nonlinear model of the synchronous generator connected to an infinite bus in the usual state space form

$$
\begin{aligned}
& \dot{E}_{q}^{\prime}=f_{11} E_{q}^{\prime}+f_{12} \cos (\delta-\alpha)+f_{13} \sin (\delta-\alpha)+g_{11} E_{F D} \\
& \begin{aligned}
& \dot{\omega}= f_{21} E_{q}^{\prime 2}+f_{22} E_{q}^{\prime} \cos (\delta-\alpha)+f_{23} E_{q}^{\prime} \sin (\delta-\alpha)+f_{24} \sin (\delta-\alpha) \cos (\delta-\alpha) \\
& \quad+f_{25} \cos ^{2}(\delta-\alpha)+f_{26} \sin ^{2}(\delta-\alpha)+f_{27} \omega+f_{28} T_{m} \\
& \dot{\delta}=\omega-1
\end{aligned}
\end{aligned}
$$

For the reduced order generator-turbine system we use the same turbine-governor model that we used for the truth model. The model of the turbine-governor system as given in Equation (2.46) and Equation (2.47) in per unit is

$$
\begin{aligned}
& \dot{T}_{m}=-\frac{1}{\tau_{T}} T_{m}+\frac{K_{T}}{\tau_{T}} G_{V} \\
& \dot{G}_{V}=-\frac{K_{G}}{\tau_{G} R_{T}} \omega-\frac{1}{\tau_{G}} G_{V}+\frac{K_{G}}{\tau_{G}} u_{T}
\end{aligned}
$$

The reduced order model of the generator-turbine system connected to an infinite bus consists of 3 nonlinear differential equations of the synchronous generator and 2 linear differential equations of the turbine-governor system. Thus, the combined system consists of 5 differential equations. Combining Equation (3.20) and Equation (3.21) the reduced order model of the synchronous generator and turbine connected to an infinite bus can be written as

$$
\begin{aligned}
& \dot{E}_{q}^{\prime}=f_{11} E_{q}^{\prime}+f_{12} \cos (\delta-\alpha)+f_{13} \sin (\delta-\alpha)+g_{11} E_{F D} \\
& \begin{aligned}
\dot{\omega}= & f_{21} E_{q}^{\prime 2}+f_{22} E_{q}^{\prime} \cos (\delta-\alpha)+f_{23} E_{q}^{\prime} \sin (\delta-\alpha)+f_{24} \sin (\delta-\alpha) \cos (\delta-\alpha) \\
\quad & \quad+f_{25} \cos ^{2}(\delta-\alpha)+f_{26} \sin ^{2}(\delta-\alpha)+f_{27} \omega+f_{28} T_{m}
\end{aligned} \\
& \dot{\delta}=\omega-1 \\
& \dot{T}_{m}=f_{41} T_{m}+f_{42} G_{V} \\
& \dot{G}_{V}=f_{51} \omega+f_{52} G_{V}+g_{55} u_{T}
\end{aligned}
$$


where, $-\frac{1}{\tau_{T}}=f_{41}, \frac{K_{T}}{\tau_{T}}=f_{42},-\frac{K_{G}}{\tau_{G} R_{T}}=f_{51},-\frac{1}{\tau_{G}}=f_{52}$, and $\frac{K_{G}}{\tau_{G}}=g_{55}$.

Let us define the state variables as $x=\left[E_{q}^{\prime}, \omega, \delta, T_{m}, G_{V}\right]^{\mathrm{T}}$, and the two control inputs as $u=\left[u_{1}, u_{2}\right]^{\mathrm{T}}=$ $\left[E_{F D}, u_{T}\right]^{\mathrm{T}}$. We can then express the simplified fifth order nonlinear model of the synchronous generator and turbine connected to an infinite bus as

$$
\begin{aligned}
\dot{x}_{1}= & f_{11} x_{1}+f_{12} \cos \left(x_{3}-\alpha\right)+f_{13} \sin \left(x_{3}-\alpha\right)+g_{11} u_{1} \\
\dot{x}_{2}= & f_{21} x_{1}^{2}+f_{22} x_{1} \cos \left(x_{3}-\alpha\right)+f_{23} x_{1} \sin \left(x_{3}-\alpha\right)+f_{24} \sin \left(x_{3}-\alpha\right) \cos \left(x_{3}-\alpha\right) \\
& +f_{25} \cos ^{2}\left(x_{3}-\alpha\right)+f_{26} \sin ^{2}\left(x_{3}-\alpha\right)+f_{27} x_{2}+f_{28} x_{4} \\
\dot{x}_{3}= & x_{2}-1 \\
\dot{x}_{4}= & f_{41} x_{4}+f_{42} x_{5} \\
\dot{x}_{5}= & f_{51} x_{2}+f_{52} x_{5}+g_{55} u_{2}
\end{aligned}
$$

Thus, we can put the simplified fifth order nonlinear model of the synchronous generator and turbine connected to an infinite bus in the usual state-space form

$$
\begin{aligned}
& \dot{x}=f(x)+g(x) u \\
& y=h(x)
\end{aligned}
$$

where

$$
\begin{aligned}
f(x) & =\left(\begin{array}{c}
f_{11} x_{1}+f_{12} \cos \left(x_{3}-\alpha\right)+f_{13} \sin \left(x_{3}-\alpha\right) \\
f_{21} x_{1}^{2}+f_{22} x_{1} \cos \left(x_{3}-\alpha\right)+f_{23} x_{1} \sin \left(x_{3}-\alpha\right)+f_{24} \sin \left(x_{3}-\alpha\right) \cos \left(x_{3}-\alpha\right) \cdots \\
\cdots+f_{25} \cos ^{2}\left(x_{3}-\alpha\right)+f_{26} \sin ^{2}\left(x_{3}-\alpha\right)+f_{27} x_{2}+f_{28} x_{4} \\
x_{2}-1 \\
f_{41} x_{4}+f_{42} x_{5} \\
f_{51} x_{2}+f_{52} x_{5}
\end{array}\right. \\
g(x)= &
\end{aligned}
$$

In Equation (3.24) and Equation (3.25), the state variables are $x=\left[E_{q}^{\prime}, \omega, \delta, T_{m}, G_{V}\right]^{\mathrm{T}}$, the two control inputs are $u=\left[E_{F D}, u_{T}\right]^{\mathrm{T}}$, and the two regulated outputs are $y=\left[V_{t}, \omega\right]^{\mathrm{T}}$. The expression for the generator terminal voltage $V_{t}$ will be derived in the next subsection.

\subsection{Derivation of the Output Generator Terminal Voltage for the Reduced Order Model}

The reduced order model of the synchronous generator and turbine connected to an infinite bus is a MIMO system with two inputs: excitation field EMF $E_{F D}$, and turbine valve control $u_{T}$, and two outputs: generator terminal voltage $V_{t}$ and rotor angle $\delta$. In this section we derive an expression for the generator terminal voltage $V_{t}$ which is the first output of the MIMO system. From Equation (3.4) the direct axis and quadrature axis stator voltage equations of a synchronous generator are given by

$$
\begin{aligned}
& V_{d}=-r I_{d}-L_{q} I_{q} \\
& V_{q}=-r I_{q}+L_{d}^{\prime} I_{d}+E_{q}^{\prime}
\end{aligned}
$$

Since the stator resistance $r \approx 0$ we can write

$$
\begin{aligned}
& V_{d}=-L_{q} I_{q} \\
& V_{q}=L_{d}^{\prime} I_{d}+E_{q}^{\prime}
\end{aligned}
$$


Substituting $I_{d}$ and $I_{q}$ as given in Equation (3.8) and Equation (3.11) in Equation (3.27) we get

$$
\begin{aligned}
V_{d}= & -L_{q} I_{q} \\
= & -\frac{L_{q}\left(r+R_{e}\right)}{\left(r+R_{e}\right)^{2}+\left(L_{d}^{\prime}+L_{e}\right)\left(L_{q}+L_{e}\right)} E_{q}^{\prime} \\
& +\frac{V_{\infty} L_{q}\left(r+R_{e}\right)}{\left(r+R_{e}\right)^{2}+\left(L_{d}^{\prime}+L_{e}\right)\left(L_{q}+L_{e}\right)} \cos (\delta-\alpha) \\
& -\frac{V_{\infty} L_{q}\left(L_{d}^{\prime}+L_{e}\right)}{\left(r+R_{e}\right)^{2}+\left(L_{d}^{\prime}+L_{e}\right)\left(L_{q}+L_{e}\right)} \sin (\delta-\alpha)
\end{aligned}
$$

Let us denote

$$
\begin{aligned}
& -\frac{L_{q}\left(r+R_{e}\right)}{\left(r+R_{e}\right)^{2}+\left(L_{d}^{\prime}+L_{e}\right)\left(L_{q}+L_{e}\right)}=V_{d 1} \\
& \frac{V_{\infty} L_{q}\left(r+R_{e}\right)}{\left(r+R_{e}\right)^{2}+\left(L_{d}^{\prime}+L_{e}\right)\left(L_{q}+L_{e}\right)}=V_{d 2} \\
& -\frac{V_{\infty} L_{q}\left(L_{d}^{\prime}+L_{e}\right)}{\left(r+R_{e}\right)^{2}+\left(L_{d}^{\prime}+L_{e}\right)\left(L_{q}+L_{e}\right)}=V_{d 3}
\end{aligned}
$$

Thus, Equation (3.28) can be rewritten as

$$
V_{d}=V_{d 1} E_{q}^{\prime}+V_{d 2} \cos (\delta-\alpha)+V_{d 3} \sin (\delta-\alpha)
$$

Now let us derive the expression for $V_{q}$

$$
\begin{aligned}
V_{q}= & L_{d}^{\prime} I_{d}+E_{q}^{\prime} \\
= & -\frac{L_{d}^{\prime}\left(L_{q}+L_{e}\right)}{\left(r+R_{e}\right)^{2}+\left(L_{d}^{\prime}+L_{e}\right)\left(L_{q}+L_{e}\right)} E_{q}^{\prime} \\
& +\frac{V_{\infty} L_{d}^{\prime}\left(L_{q}+L_{e}\right)}{\left(r+R_{e}\right)^{2}+\left(L_{d}^{\prime}+L_{e}\right)\left(L_{q}+L_{e}\right)} \cos (\delta-\alpha) \\
& +\frac{V_{\infty} L_{d}^{\prime}\left(r+R_{e}\right)}{\left(r+R_{e}\right)^{2}+\left(L_{d}^{\prime}+L_{e}\right)\left(L_{q}+L_{e}\right)} \sin (\delta-\alpha)+E_{q}^{\prime}
\end{aligned}
$$

Let us denote

$$
\begin{aligned}
& -\frac{L_{d}^{\prime}\left(L_{q}+L_{e}\right)}{\left(r+R_{e}\right)^{2}+\left(L_{d}^{\prime}+L_{e}\right)\left(L_{q}+L_{e}\right)}=V_{q 1} \\
& \frac{V_{\infty} L_{d}^{\prime}\left(L_{q}+L_{e}\right)}{\left(r+R_{e}\right)^{2}+\left(L_{d}^{\prime}+L_{e}\right)\left(L_{q}+L_{e}\right)}=V_{q 2} \\
& \frac{V_{\infty} L_{d}^{\prime}\left(r+R_{e}\right)}{\left(r+R_{e}\right)^{2}+\left(L_{d}^{\prime}+L_{e}\right)\left(L_{q}+L_{e}\right)}=V_{q 3}
\end{aligned}
$$

Thus, Equation (3.31) can be rewritten as

$$
V_{q}=V_{q 1} E_{q}^{\prime}+V_{q 2} \cos (\delta-\alpha)+V_{q 3} \sin (\delta-\alpha)+E_{q}^{\prime}
$$

The generator terminal voltage $V_{t}$ is given by

$$
V_{t}=\sqrt{V_{d}^{2}+V_{q}^{2}}
$$

The output generator terminal voltage, $y_{1}=V_{t}$, as a function of the states $x$, is

$$
\begin{aligned}
& V_{d}=V_{d 1} x_{1}+V_{d 2} \cos \left(x_{3}-\alpha\right)+V_{d 3} \sin \left(x_{3}-\alpha\right) \\
& V_{q}=V_{q 1} x_{1}+V_{q 2} \cos \left(x_{3}-\alpha\right)+V_{q 3} \sin \left(x_{3}-\alpha\right)+x_{1} \\
& V_{t}=\sqrt{V_{d}^{2}+V_{q}^{2}}
\end{aligned}
$$


Thus, the output equation consists of a nonlinear equation for $V_{t}$ as given in Equation (3.34) and Equation (3.35), and a simple linear equation for $\omega$

$$
y=h(x)=\left[\begin{array}{l}
V_{t} \\
x_{2}
\end{array}\right]
$$

\subsection{Linearization of the Reduced Order Model by Taylor series approximation}

In this section we linearize the fifth-order nonlinear model of the synchronous generator and turbine connected to an infinite bus by using the Taylor series approximation about a nominal operating point $\left(x_{0}, u_{0}\right)$. The operating condition is a steady state equilibrium of the system. The steady state equilibrium condition is attained by the system after all the transients die out or decay to zero. The equilibrium point $\left(x_{0}, u_{0}\right)$ is computed by solving the differential equation, $\dot{x}=f\left(x_{0}\right)+g\left(x_{0}\right) u_{0}=0$. From Equation (3.25) we can write

$$
f_{1}(x)=f_{11} x_{1}+f_{12} \cos \left(x_{3}-\alpha\right)+f_{13} \sin \left(x_{3}-\alpha\right)
$$

Therefore,

$$
\begin{gathered}
\left.\frac{\partial f_{1}}{\partial x_{1}}\right|_{x_{0}}=f_{11} \\
\left.\frac{\partial f_{1}}{\partial x_{2}}\right|_{x_{0}}=0 \\
\left.\frac{\partial f_{1}}{\partial x_{3}}\right|_{x_{0}}=-f_{12} \sin \left(x_{30}-\alpha\right)+f_{13} \cos \left(x_{30}-\alpha\right) \\
\left.\frac{\partial f_{1}}{\partial x_{4}}\right|_{x_{0}}=0 \\
\left.\frac{\partial f_{1}}{\partial x_{5}}\right|_{x_{0}}=0 \\
f_{2}(x)=f_{21} x_{1}^{2}+f_{22} x_{1} \cos \left(x_{3}-\alpha\right)+f_{23} x_{1} \sin \left(x_{3}-\alpha\right)+f_{24} \sin \left(x_{3}-\alpha\right) \cos \left(x_{3}-\alpha\right) \\
+f_{25} \cos ^{2}\left(x_{3}-\alpha\right)+f_{26} \sin ^{2}\left(x_{3}-\alpha\right)+f_{27} x_{2}+f_{28} x_{4}
\end{gathered}
$$

Therefore,

$$
\begin{aligned}
& \left.\frac{\partial f_{2}}{\partial x_{1}}\right|_{x_{0}}=2 f_{21} x_{10}+f_{22} \cos \left(x_{30}-\alpha\right)+f_{23} \sin \left(x_{30}-\alpha\right) \\
& \left.\frac{\partial f_{2}}{\partial x_{2}}\right|_{x_{0}}=f_{27} \\
& \left.\frac{\partial f_{2}}{\partial x_{3}}\right|_{x_{0}}=-f_{22} x_{10} \sin \left(x_{30}-\alpha\right)+f_{23} x_{10} \cos \left(x_{30}-\alpha\right)+f_{24} \cos 2\left(x_{30}-\alpha\right)-f_{25} \sin 2\left(x_{30}-\alpha\right)+f_{26} \sin 2\left(x_{30}-\alpha\right) \\
& \left.\frac{\partial f_{2}}{\partial x_{4} 0}\right|_{x_{0}}=f_{28} \\
& \left.\frac{\partial f_{2}}{\partial x_{5}}\right|_{x_{0}}=0
\end{aligned}
$$

$$
f_{3}(x)=x_{2}-1
$$


Therefore,

$$
\begin{gathered}
\left.\frac{\partial f_{3}}{\partial x_{1}}\right|_{x_{0}}=0 \\
\left.\frac{\partial f_{3}}{\partial x_{2}}\right|_{x_{0}}=1 \\
\left.\frac{\partial f_{3}}{\partial x_{3}}\right|_{x_{0}}=0 \\
\left.\frac{\partial f_{3}}{\partial x_{4}}\right|_{x_{0}}=0 \\
\left.\frac{\partial f_{3}}{\partial x_{5}}\right|_{x_{0}}=0 \\
f_{4}(x)=f_{41} x_{4}+f_{42} x_{5}
\end{gathered}
$$

Therefore,

$$
\begin{gathered}
\left.\frac{\partial f_{4}}{\partial x_{1}}\right|_{x_{0}}=0 \\
\left.\frac{\partial f_{4}}{\partial x_{2}}\right|_{x_{0}}=0 \\
\left.\frac{\partial f_{4}}{\partial x_{3}}\right|_{x_{0}}=0 \\
\left.\frac{\partial f_{4}}{\partial x_{4}}\right|_{x_{0}}=f_{41} \\
\left.\frac{\partial f_{4}}{\partial x_{5}}\right|_{x_{0}}=f_{42} \\
f_{5}(x)=f_{51} x_{2}+f_{52} x_{5}
\end{gathered}
$$

Therefore,

$$
\begin{aligned}
& \left.\frac{\partial f_{5}}{\partial x_{1}}\right|_{x_{0}}=0 \\
& \left.\frac{\partial f_{5}}{\partial x_{2}}\right|_{x_{0}}=f_{51} \\
& \left.\frac{\partial f_{5}}{\partial x_{3}}\right|_{x_{0}}=0 \\
& \left.\frac{\partial f_{5}}{\partial x_{4}}\right|_{x_{0}}=0 \\
& \left.\frac{\partial f_{5}}{\partial x_{5}}\right|_{x_{0}}=f_{52} \\
& g_{1}(x)=g_{11} u_{1}
\end{aligned}
$$

Therefore,

$$
\begin{aligned}
& \left.\frac{\partial g_{1}}{\partial u_{1}}\right|_{u_{0}}=g_{11} \\
& \left.\frac{\partial g_{1}}{\partial u_{2}}\right|_{u_{0}}=0
\end{aligned}
$$




$$
g_{2}(x)=0
$$

Therefore,

$$
\begin{aligned}
& \left.\frac{\partial g_{2}}{\partial u_{1}}\right|_{u_{0}}=0 \\
& \left.\frac{\partial g_{2}}{\partial u_{2}}\right|_{u_{0}}=0 \\
& g_{3}(x)=0
\end{aligned}
$$

Therefore,

$$
\begin{aligned}
& \left.\frac{\partial g_{3}}{\partial u_{1}}\right|_{u_{0}}=0 \\
& \left.\frac{\partial g_{3}}{\partial u_{2}}\right|_{u_{0}}=0 \\
& g_{4}(x)=0
\end{aligned}
$$

Therefore,

$$
\begin{gathered}
\left.\frac{\partial g_{4}}{\partial u_{1}}\right|_{u_{0}}=0 \\
\left.\frac{\partial g_{4}}{\partial u_{2}}\right|_{u_{0}}=0 \\
g_{5}(x)=g_{55} u_{2}
\end{gathered}
$$

Therefore,

$$
\begin{aligned}
& \left.\frac{\partial g_{5}}{\partial u_{1}}\right|_{u_{0}}=0 \\
& \left.\frac{\partial g_{5}}{\partial u_{2}}\right|_{u_{0}}=g_{55}
\end{aligned}
$$

Therefore, the linear reduced order model of the synchronous generator and turbine-governor system connected to an infinite bus is

$$
\dot{\mathbf{x}}=A \mathbf{x}+B \mathbf{u}
$$

where

$$
\begin{aligned}
\mathbf{x}^{T} & =\left[\begin{array}{lllll}
\Delta E_{q}^{\prime} & \Delta \omega & \Delta \delta & \Delta T_{m} & \Delta G_{V}
\end{array}\right] \\
\mathbf{u} & =\left[\begin{array}{c}
\Delta E_{F D} \\
\Delta u_{T}
\end{array}\right]
\end{aligned}
$$

In the above equation $\Delta$ is the deviation from the nominal operating condition, i.e. $E_{q}^{\prime}-E_{q 0}^{\prime}=\Delta E_{q}^{\prime}$, $\omega-\omega_{0}=\Delta \omega, \delta-\delta_{0}=\Delta \delta, T_{m}-T_{m 0}=\Delta T_{m}, G_{V}-G_{V 0}=\Delta G_{V}, E_{F D}-E_{F D 0}=\Delta E_{F D}$, and $u_{T}-u_{T 0}=\Delta u_{T}$, 
and

$$
A=\left[\begin{array}{lllll}
\frac{\partial f_{1}}{\partial x_{1}} & \frac{\partial f_{1}}{\partial x_{2}} & \frac{\partial f_{1}}{\partial x_{3}} & \frac{\partial f_{1}}{\partial x_{4}} & \frac{\partial f_{1}}{\partial x_{5}} \\
\frac{\partial f_{2}}{\partial x_{1}} & \frac{\partial f_{2}}{\partial x_{2}} & \frac{\partial f_{2}}{\partial x_{3}} & \frac{\partial f_{2}}{\partial x_{4}} & \frac{\partial f_{2}}{\partial x_{5}} \\
\frac{\partial f_{3}}{\partial x_{1}} & \frac{\partial f_{3}}{\partial x_{2}} & \frac{\partial f_{3}}{\partial x_{3}} & \frac{\partial f_{3}}{\partial x_{4}} & \frac{\partial f_{3}}{\partial x_{5}} \\
\frac{\partial f_{4}}{\partial x_{1}} & \frac{\partial f_{4}}{\partial x_{2}} & \frac{\partial f_{4}}{\partial x_{3}} & \frac{\partial f_{4}}{\partial x_{4}} & \frac{\partial f_{4}}{\partial x_{5}} \\
\frac{\partial f_{5}}{\partial x_{1}} & \frac{\partial f_{5}}{\partial x_{2}} & \frac{\partial f_{5}}{\partial x_{3}} & \frac{\partial f_{5}}{\partial x_{4}} & \frac{\partial f_{5}}{\partial x_{5}}
\end{array}\right]_{x_{0}}
$$

In order to design a linear controller for the linearized model obtained by using Taylor series approximation, we need to find an expression for the first output $\Delta V_{t}$. By using Taylor series approximation, we have

$$
V_{t}(x)=V_{t}\left(x_{0}\right)+\left.\frac{\mathrm{d} V_{t}}{\mathrm{~d} x}\right|_{x_{0}}\left(x-x_{0}\right)+\ldots
$$

Where, $x=\left[E_{q}^{\prime}, \omega, \delta, T_{m}, G_{V}\right]^{\mathrm{T}}$, are the states of the reduced order nonlinear model. Let us denote $V_{t}(x)-$ $V_{t}\left(x_{0}\right)=\Delta V_{t}$, and $V_{t}\left(x_{0}\right)=V_{t 0}$. By neglecting the second and higher order derivatives in Equation (3.61) we have

$$
\Delta V_{t}=\left.\frac{\mathrm{d} V_{t}}{\mathrm{~d} x}\right|_{x_{0}}\left(x-x_{0}\right)
$$

From Equation (3.34) and Equation (3.35) the generator terminal voltage $V_{t}$ is given by

$$
V_{t}=\sqrt{V_{d}^{2}+V_{q}^{2}}
$$


where

$$
\begin{aligned}
& V_{d}=V_{d 1} E_{q}^{\prime}+V_{d 2} \cos (\delta-\alpha)+V_{d 3} \sin (\delta-\alpha) \\
& V_{q}=V_{q 1} E_{q}^{\prime}+V_{q 2} \cos (\delta-\alpha)+V_{q 3} \sin (\delta-\alpha)+E_{q}^{\prime}
\end{aligned}
$$

We can write

$$
\Delta V_{t}=\left.\frac{\mathrm{d} V_{t}}{\mathrm{~d} x}\right|_{x_{0}}\left(x-x_{0}\right)=\left.\left(\frac{\partial V_{t}}{\partial V_{d}} \frac{\mathrm{d} V_{d}}{\mathrm{~d} x}\right)\right|_{x_{0}}\left(x-x_{0}\right)+\left.\left(\frac{\partial V_{t}}{\partial V_{q}} \frac{\mathrm{d} V_{q}}{\mathrm{~d} x}\right)\right|_{x_{0}}\left(x-x_{0}\right)
$$

Differentiating Equation (3.63) with respect to $V_{d}$ and $V_{q}$ respectively

$$
\begin{aligned}
& \left.\frac{\partial V_{t}}{\partial V_{d}}\right|_{x_{0}}=\frac{V_{d 0}}{\sqrt{V_{d 0}^{2}+V_{q 0}^{2}}}=\frac{V_{d 0}}{V_{t 0}} \\
& \left.\frac{\partial V_{t}}{\partial V_{q}}\right|_{x_{0}}=\frac{V_{q 0}}{\sqrt{V_{d 0}^{2}+V_{q 0}^{2}}}=\frac{V_{q 0}}{V_{t 0}}
\end{aligned}
$$

Also

$$
\begin{aligned}
& \left.\frac{\mathrm{d} V_{d}}{\mathrm{~d} x}\right|_{x_{0}}\left(x-x_{0}\right)=\left.\left(E_{q}^{\prime}-E_{q 0}^{\prime}\right) \frac{\partial V_{d}}{\partial E_{q}^{\prime}}\right|_{x_{0}}+\left.\left(\omega-\omega_{0}\right) \frac{\partial V_{d}}{\partial \omega}\right|_{x_{0}}+\left.\left(\delta-\delta_{0}\right) \frac{\partial V_{d}}{\partial \delta}\right|_{x_{0}}+\left.\left(T_{m}-T_{m 0}\right) \frac{\partial V_{d}}{\partial T_{m}}\right|_{x_{0}}+\left.\left(G_{V}-G_{V 0}\right) \frac{\partial V_{d}}{\partial G_{V}}\right|_{x_{0}} \\
& \left.\frac{\mathrm{d} V_{q}}{\mathrm{~d} x}\right|_{x_{0}}\left(x-x_{0}\right)=\left.\left(E_{q}^{\prime}-E_{q 0}^{\prime}\right) \frac{\partial V_{q}}{\partial E_{q}^{\prime}}\right|_{x_{0}}+\left.\left(\omega-\omega_{0}\right) \frac{\partial V_{q}}{\partial \omega}\right|_{x_{0}}+\left.\left(\delta-\delta_{0}\right) \frac{\partial V_{q}}{\partial \delta}\right|_{x_{0}}+\left.\left(T_{m}-T_{m 0}\right) \frac{\partial V_{q}}{\partial T_{m}}\right|_{x_{0}}+\left.\left(G_{V}-G_{V 0}\right) \frac{\partial V_{q}}{\partial G_{V}}\right|_{x_{0}}
\end{aligned}
$$

Let us recall that the deviations of the state variables form their nominal operating condition are given by: $E_{q}^{\prime}-E_{q 0}^{\prime}=\Delta E_{q}^{\prime}, \omega-\omega_{0}=\Delta \omega, \delta-\delta_{0}=\Delta \delta, T_{m}-T_{m 0}=\Delta T_{m}, G_{V}-G_{V 0}=\Delta G_{V}$. Simplifying Equation (3.67) we get

$$
\begin{aligned}
& \left.\frac{\mathrm{d} V_{d}}{\mathrm{~d} x}\right|_{x_{0}}\left(x-x_{0}\right)=V_{d 1} \Delta E_{q}^{\prime}-V_{d 2} \sin \left(\delta_{\circ}-\alpha\right) \Delta \delta+V_{d 3} \cos \left(\delta_{\circ}-\alpha\right) \Delta \delta \\
& \left.\frac{\mathrm{d} V_{q}}{\mathrm{~d} x}\right|_{x_{0}}\left(x-x_{0}\right)=V_{q 1} \Delta E_{q}^{\prime}-V_{q 2} \sin \left(\delta_{\circ}-\alpha\right) \Delta \delta+V_{q 3} \cos \left(\delta_{\circ}-\alpha\right) \Delta \delta+\Delta E_{q}^{\prime}
\end{aligned}
$$

Substituting Equation (3.66) and Equation (3.68) in Equation (3.65) and rearranging we get

$$
\begin{aligned}
\Delta V_{t}= & \left(\frac{V_{d 0}}{V_{t 0}} V_{d 1}+\frac{V_{q 0}}{V_{t 0}} V_{q 1}+\frac{V_{q 0}}{V_{t 0}}\right) \Delta E_{q}^{\prime} \\
& +\left(-\frac{V_{d 0}}{V_{t 0}} V_{d 2} \sin \left(\delta_{\circ}-\alpha\right)+\frac{V_{d 0}}{V_{t 0}} V_{d 3} \cos \left(\delta_{\circ}-\alpha\right)-\frac{V_{q 0}}{V_{t 0}} V_{q 2} \sin \left(\delta_{\circ}-\alpha\right)+\frac{V_{q 0}}{V_{t 0}} V_{q 3} \cos \left(\delta_{\circ}-\alpha\right)\right) \Delta \delta
\end{aligned}
$$

Let us denote

$$
\begin{aligned}
& \left(\frac{V_{d 0}}{V_{t 0}} V_{d 1}+\frac{V_{q 0}}{V_{t 0}} V_{q 1}+\frac{V_{q 0}}{V_{t 0}}\right)=T_{1} \\
& \left(-\frac{V_{d 0}}{V_{t 0}} V_{d 2} \sin \left(\delta_{\circ}-\alpha\right)+\frac{V_{d 0}}{V_{t 0}} V_{d 3} \cos \left(\delta_{\circ}-\alpha\right)-\frac{V_{q 0}}{V_{t 0}} V_{q 2} \sin \left(\delta_{\circ}-\alpha\right)+\frac{V_{q 0}}{V_{t 0}} V_{q 3} \cos \left(\delta_{\circ}-\alpha\right)\right)=T_{2}
\end{aligned}
$$

Thus, Equation (3.69) can be simplified to get

$$
\Delta V_{t}=T_{1} \Delta E_{q}^{\prime}+T_{2} \Delta \delta
$$

Thus, the linearized output equation of the reduced order model is given by

$$
\mathbf{y}=C \mathbf{x}
$$


where

$$
\mathbf{y}=\left[\begin{array}{c}
\Delta V_{t} \\
\Delta \omega
\end{array}\right]
$$

and the output matrix $C$ is

$$
C=\left[\begin{array}{ccccc}
T_{1} & 0 & T_{2} & 0 & 0 \\
0 & 1 & 0 & 0 & 0
\end{array}\right]
$$

\subsection{Example}

In this section we calculate the $(A, B, C)$ system matrices of the truth model and the reduced order model linearized about a given nominal operating point. The power, voltage, and current ratings as well as the parameters of the synchronous generator are given in Table 3.1, which contains values for an actual synchronous generator with some quantities, denoted by an asterisk, being estimated for our study [1]. The power rating of a synchronous generator is equal to the product of the voltage per phase, the current per phase, and the number of phases. It is normally stated in megavolt-amperes (MVA) for large generators. The parameters given in Table 3.1 are not in the per unit system. The quantities in Table 3.1 are converted to the per unit system, which are given in Table 3.2 [1]. Table 3.3 gives the parameters of the reduced order nonlinear model in the per unit system.

Before we proceed to the calculation of the system matrices, we briefly explain a basic power system terminology, that is used in determining a suitable operating condition. Real power (also known as active power) $(P)$, measured in watts $(\mathrm{W})$; apparent power $(S)$, measured in volt-amperes $(\mathrm{VA})$; and reactive power $(Q)$, measured in reactive volt-amperes (var) are related by the expression, $S=P+j Q$ in vector form. If $\phi$ is the phase angle between the current and voltage, then the power factor $(\mathrm{PF})$ is equal to the cosine of the angle, $|\cos \phi|$, and $|P|=|S| \cos \phi$ and $|Q|=|S| \sin \phi$. Power factors are usually stated as "leading" or "lagging" to show the sign of the phase angle $\phi$. If a purely resistive load is connected to a power supply, current and voltage will change polarity in step, the power factor will be unity, and the electrical energy flows in a single direction across the network in each cycle. Inductive loads such as transformers and motors (any type of wound coil) consume reactive power with current waveform lagging the voltage. Capacitive loads such as capacitor banks or buried cable generate reactive power with current phase leading the voltage. Both types of loads will absorb energy during part of the AC cycle, which is stored in the device's magnetic or electric field, only to return this energy back to the source during the rest of the cycle. Since a majority of the loads in a power grid are inductive, we assume power factor lagging conditions, where the generator armature current lags the generator terminal voltage. Also a high power factor is generally desirable in a transmission system to reduce transmission losses and improve voltage regulation at the load. A power factor of 0.85 is assumed, which is within the stable operating limits of a synchronous generator.

The synchronous generator is connected to an infinite bus through a transmission line having $R_{e}=0.02$ p.u., and $L_{e}=0.4$ p.u. The infinite bus voltage is 1.0 p.u. The machine loading is given by, real power $P=1.0$ p.u. at $0.85 \mathrm{PF}$ lagging conditions. The steady state operating conditions of a synchronous generator turbine system connected to an infinite bus depend on the synchronous generator turbine system parameters, transmission line parameters, and the machine loading. For the synchronous generator with parameters and loading conditions given in Table 3.1 and Table 3.2, a steady state operating point for the truth model is 
evaluated which is as given in [1]:

$$
\begin{aligned}
& I_{d 0}=-0.9185 \\
& I_{F 0}=1.6315 \\
& I_{D 0}=-4.6204 \times 10^{-6} \\
& I_{q 0}=0.4047 \\
& I_{Q 0}=5.9539 \times 10^{-5} \\
& \omega_{0}=1 \\
& \delta_{0}=1 \\
& T_{m 0}=1.0012 \\
& G_{V 0}=1.0012 \\
& V_{q 0}=0.9670 \\
& V_{d 0}=-0.6628 \\
& V_{t 0}=1.172 \\
& \delta_{0}-\alpha=53.736^{\circ}
\end{aligned}
$$

Table 3.1: Ratings and Parameters of the Synchronous Generator

\begin{tabular}{|c||c|c|c|c|}
\hline Variables & Rated MVA & Rated voltage & Excitation voltage & Stator current \\
\hline Values & $160 \mathrm{MVA}$ & $15 \mathrm{kV}, \mathrm{Y}$ connected & $375 \mathrm{~V}$ & $6158.40 \mathrm{~A}$ \\
\hline \hline Variables & Field current & Power factor & $L_{d}$ & $L_{F}$ \\
\hline Values & $926 \mathrm{~A}$ & 0.85 & $6.341 \times 10^{-3} \mathrm{H}$ & $2.189 \mathrm{H}$ \\
\hline \hline Variables & $L_{D}$ & $L_{q}$ & $L_{Q}$ & $k M_{F}$ \\
\hline Values & $5.989 \times 10^{-3} \mathrm{H}^{*}$ & $6.118 \times 10^{-3} \mathrm{H}$ & $1.423 \times 10^{-3} \mathrm{H}^{*}$ & $109.01 \times 10^{-3} \mathrm{H}^{*}$ \\
\hline \hline Variables & $k M_{D}$ & $M_{R}$ & $k M_{Q}$ & $r\left(125^{\circ} \mathrm{C}\right)$ \\
\hline Values & $5.782 \times 10^{-3} \mathrm{H}^{*}$ & $109.01 \times 10^{-3} \mathrm{H}^{*}$ & $2.779 \times 10^{-3} \mathrm{H}^{*}$ & $1.542 \times 10^{-3} \Omega$ \\
\hline \hline Variables & $r_{F}\left(125^{\circ} \mathrm{C}\right)$ & $r_{D}$ & $r_{Q}$ & Inertia constant $H$ \\
\hline Values & $0.371 \Omega$ & $18.421 \times 10^{-3} \Omega^{*}$ & $18.969 \times 10^{-3} \Omega^{*}$ & $1.765 \frac{\mathrm{kW} \cdot \mathrm{s}}{\mathrm{h} p}$ \\
\hline \hline Variables & $R_{e}$ & $L_{e}$ & $D$ & $\omega_{0}($ in rads $)$ \\
\hline Values & $0.02 \mathrm{p} . \mathrm{u}$. & $0.4 \mathrm{p} . \mathrm{u}$. & 0 & 376.99 \\
\hline
\end{tabular}

Table 3.2: Parameters of the Truth model of the Synchronous Generator-Turbine System in p.u.

\begin{tabular}{|c||c|c|c|c|c|c|}
\hline Variables (in p.u.) & $L_{d}$ & $L_{F}$ & $L_{D}$ & $L_{q}$ & $L_{Q}$ & $k M_{F}$ \\
\hline Values & 1.70 & 1.65 & 1.605 & 1.64 & 1.526 & 1.55 \\
\hline \hline Variables (in p.u.) & $k M_{D}$ & $M_{R}$ & $k M_{Q}$ & $r$ & $r_{F}$ & $r_{D}$ \\
\hline Values & 1.55 & 1.55 & 1.49 & 0.001096 & 0.000742 & 0.0131 \\
\hline \hline Variables (in p.u.) & $r_{Q}$ & $H$ (in s) & $R_{e}$ & $L_{e}$ & $D$ & $\omega_{0}$ (in rads) \\
\hline Values & 0.0540 & 2.37 & 0.02 & 0.4 & 0 & 376.99 \\
\hline Variables (in p.u.) & $K_{T}$ & $K_{G}$ & $\tau_{T}$ & $\tau_{G}$ & $R_{T}$ & $k$ \\
\hline Values & 1 & 1 & 0.5 & 0.2 & 20 & $\sqrt{3 / 2}$ \\
\hline Variables (in p.u.) & $L_{d}^{\prime}$ & $\tau_{d 0}^{\prime}$ & $\tau_{j}$ & $V_{\infty}$ & $\alpha$ & \\
\hline Values & 0.245 & 5.9 & 4.74 & 1.00 & $3.5598^{\circ}$ & \\
\hline
\end{tabular}


Table 3.3: Parameters of the Reduced Order Nonlinear Model of the SMIB in p.u.

\begin{tabular}{|c||c|c|c|c|c|c|}
\hline Variables (in p.u.) & $V_{d 1}$ & $V_{d 2}$ & $V_{d 3}$ & $V_{q 1}$ & $V_{q 2}$ & $V_{q 3}$ \\
\hline Values & -0.0249 & 0.0249 & -0.8037 & -0.3797 & 0.3797 & 0.0037 \\
\hline \hline Variables (in p.u.) & $f_{11}$ & $f_{12}$ & $f_{13}$ & $f_{21}$ & $f_{22}$ & $f_{23}$ \\
\hline Values & -0.5517 & 0.3822 & 0.0037 & -0.0101 & 0.0171 & -0.3269 \\
\hline \hline Variables (in p.u.) & $f_{24}$ & $f_{25}$ & $f_{26}$ & $f_{27}$ & $f_{28}$ & $f_{41}$ \\
\hline Values & 0.2235 & -0.0069 & 0.0022 & 0 & 0.2110 & -2 \\
\hline \hline Variables (in p.u.) & $f_{42}$ & $f_{51}$ & $f_{52}$ & $g_{11}$ & $g_{55}$ & $E_{F D \max }$ \\
\hline Values & 2 & -0.2500 & -5 & 0.1695 & 5 & 5 \\
\hline \hline Variables (in p.u.) & $E_{F D \min }$ & $G_{V \max }$ & $G_{V \min }$ & & & \\
\hline Values & -5 & 1.2 & 0 & & & \\
\hline
\end{tabular}

The $\mathbf{A}$ matrix of the truth model linearized about the operating point is

$$
\mathbf{A}=\left[\begin{array}{ccccccccc}
-0.0361 & 0.000437 & 0.0142 & -3.4883 & -2.5478 & -1.4119 & 1.0115 & 0 & 0 \\
0.0124 & -0.0050 & 0.0772 & 1.2022 & 0.8781 & 0.4866 & -0.3486 & 0 & 0 \\
0.0228 & 0.0044 & -0.0964 & 2.2077 & 1.6125 & 0.8936 & -0.6401 & 0 & 0 \\
3.5888 & 2.6489 & 2.6489 & -0.0361 & 0.0901 & 1.0254 & 1.3779 & 0 & 0 \\
-3.5042 & -2.5864 & -2.5864 & 0.0352 & -0.1234 & -1.0012 & -1.3454 & 0 & 0 \\
-0.000013 & -0.00035 & -0.00035 & -0.0014 & -0.00076 & 0 & 0 & 0.00056 & 0 \\
0 & 0 & 0 & 0 & 0 & 1.0000 & 0 & 0 & 0 \\
0 & 0 & 0 & 0 & 0 & 0 & 0 & -2.0000 & 2.0000 \\
0 & 0 & 0 & 0 & 0 & -0.2500 & 0 & 0 & -5.0000
\end{array}\right]
$$

The $\mathbf{B}$ matrix of the truth model linearized about the operating point is

$$
\mathbf{B}=\left[\begin{array}{cc}
-0.5893 & 0 \\
6.6918 & 0 \\
-5.8933 & 0 \\
0 & 0 \\
0 & 0 \\
0 & 0 \\
0 & 0 \\
0 & 0 \\
0 & 5.0000
\end{array}\right]
$$

the $\mathbf{C}$ matrix of the truth model linearized about the operating point is

$$
\mathbf{C}=\left[\begin{array}{ccccccccc}
0.8510 & 0.8739 & 0.8708 & 0.5673 & 0.6059 & 0.8691 & -0.1048 & 0 & 0 \\
0 & 0 & 0 & 0 & 0 & 1.0000 & 0 & 0 & 0
\end{array}\right]
$$

and the $\mathbf{D}$ matrix of the truth model linearized about the operating point is

$$
\mathbf{D}=\left[\begin{array}{cc}
0.1333 & 0 \\
0 & 0
\end{array}\right]
$$


The open loop eigenvalues of the $\mathbf{A}$ matrix of the truth model are

$$
\begin{aligned}
& -5.0000 \\
& -0.0359+0.9983 i \\
& -0.0359-0.9983 i \\
& -2.0000 \\
& -0.0016+0.0289 i \\
& -0.0016-0.0289 i \\
& -0.0007 \\
& -0.0995 \\
& -0.1217
\end{aligned}
$$

Also a steady state operating point for the reduced order nonlinear model is

$$
\begin{aligned}
& x_{10}=E_{q 0}^{\prime}=1.1925 \\
& x_{20}=\omega_{0}=1 \\
& x_{30}=\delta_{0}=1 \\
& x_{40}=T_{m 0}=1.0012 \\
& x_{50}=G_{V 0}=1.0012
\end{aligned}
$$

The $A$ matrix of the reduced order model linearized about the operating point is

$$
A=\left[\begin{array}{ccccc}
-0.5517 & 0 & -0.3060 & 0 & 0 \\
-0.2776 & 0 & -0.3054 & 0.2110 & 0 \\
0 & 1 & 0 & 0 & 0 \\
0 & 0 & 0 & -2 & 2 \\
0 & -0.25 & 0 & 0 & -5
\end{array}\right]
$$

The $B$ matrix of the reduced order model linearized about the operating point is

$$
B=\left[\begin{array}{cc}
0.1695 & 0 \\
0 & 0 \\
0 & 0 \\
0 & 0 \\
0 & 5
\end{array}\right]
$$

the $C$ matrix of the reduced order model linearized about the operating point is

$$
C=\left[\begin{array}{ccccc}
0.5258 & 0 & 0.0294 & 0 & 0 \\
0 & 1 & 0 & 0 & 0
\end{array}\right]
$$

and the $D$ matrix of the reduced order model linearized about the operating point is

$$
D=\left[\begin{array}{ll}
0 & 0 \\
0 & 0
\end{array}\right]
$$

The open loop eigenvalues of the $A$ matrix of the reduced order model are

$$
\begin{aligned}
& -5.0069 \\
& -0.1048+0.4778 i \\
& -0.1048-0.4778 i \\
& -0.3514 \\
& -1.9839
\end{aligned}
$$

From Equation (3.80) and Equation (3.86) we can see that the eigenvalues of the open loop system lie on the left half plane. 


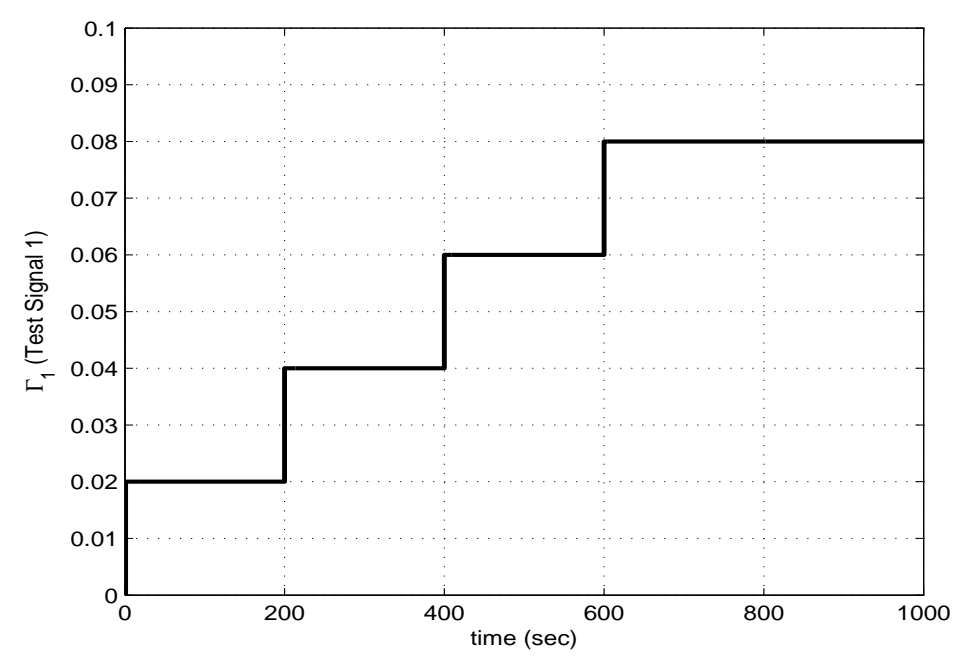

Figure 4.1: Plot of $\Gamma_{1}$ (Test Signal 1) vs time

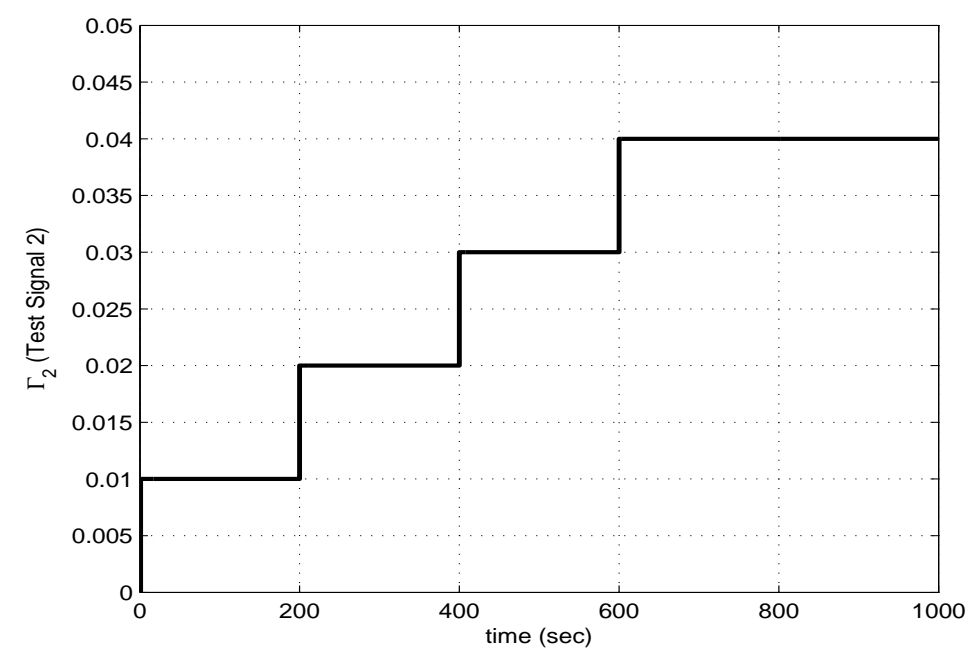

Figure 4.2: Plot of $\Gamma_{2}$ (Test Signal 2) vs time

\section{Open Loop Input-Output Behavior of the Synchronous Generator and Turbine-Governor System}

In this section we observe the open loop input-output behavior of the truth model and the reduced order nonlinear model of the SMIB for test signals given in Figure 4.1 and Figure 4.2. The first control input $V_{F}$ of the truth model and the first control input $E_{F D}$ of the reduced order model are different. As given in Equation (10.41) the field voltage, $V_{F}$, is related to excitation field emf, $E_{F D}$, by the following expression

$$
\begin{aligned}
V_{F} & =\left(\frac{r_{F}}{\omega_{R} k M_{F}}\right) E_{F D} \\
& =e_{15} E_{F D}
\end{aligned}
$$

In the above expression, $\omega_{R}=1$ p.u., and $e_{15}=\left(\frac{r_{F}}{\omega_{R} k M_{F}}\right)$.

We first test the truth model and the reduced order nonlinear model of the SMIB for $\Gamma_{1}$ (Test signal 1) given in Figure 4.1. In this case the two inputs for the truth model are, $u=\left[V_{F}, u_{T}\right]^{\mathrm{T}}=\left[e_{15} \Gamma_{1}, \Gamma_{1}\right]^{\mathrm{T}}$, and the two inputs for the reduced order nonlinear model are, $u=\left[E_{F D}, u_{T}\right]^{\mathrm{T}}=\left[\Gamma_{1}, \Gamma_{1}\right]^{\mathrm{T}}$. 
Figure 4.3, Figure 4.4, and Figure 4.5 show generator terminal voltage $V_{t}$, rotor angle $\delta$, and frequency $\omega$ vs time plots for $\Gamma_{1}$ (Test Signal 1) applied to the reduced order nonlinear model. From Figure 4.3 we can see that after the addition of the fourth step of $\Gamma_{1}$ (Test Signal 1) at 600 seconds, the generator terminal voltage $V_{t}$ settles to a new steady state value of 0.83 p.u.. From Figure 4.4 we can see that the rotor angle $\delta$ first undergoes a large undershoot, followed by oscillations about $0.1 \mathrm{p} . \mathrm{u}$. after the application of the second incremental step of $\Gamma_{1}$ at 200 seconds, which is further followed by reduced oscillations about 0.3 p.u. after the application of the third step of $\Gamma_{1}$ at 400 seconds, and finally after the application of the fourth and final step at 600 seconds the oscillations almost die out and the rotor angle $\delta$ settles to a value of 0.725 p.u.. Figure 4.5 shows that the frequency $\omega$ settles to its steady state value of 1 p.u. after the application of the fourth step at 600 seconds. Like the rotor angle $\delta$, the oscillations of the frequency $\omega$ also decrease with the application of each incremental step.

Figure 4.6, Figure 4.7, and Figure 4.8 show generator terminal voltage $V_{t}$, rotor angle $\delta$, and frequency $\omega$ vs time plots for $\Gamma_{1}$ (Test Signal 1) applied to the truth model. From Figure 4.6 we can see that the generator terminal voltage $V_{t}$ converges very slowly to the new steady state value of 0.83 p.u.. From Figure 4.7 we can see that the rotor angle $\delta$ first undergoes a large undershoot after which it oscillates about 0.1 p.u., where the oscillations decay with time. Figure 4.8 shows that the frequency $\omega$ oscillates about its steady state value of 1 p.u.. We observe a peculiar difference between the performance of the reduced order nonlinear model and the truth model, for $\Gamma_{1}$ (Test Signal 1). The addition of each incremental step of $\Gamma_{1}$ after a fixed interval has a distinct effect on the amplitude and damping of the output oscillations for the reduced order nonlinear model, where a rapid decrease in the output oscillations is observed with the application of each step. Thus, we observe transitions in the output response at time instants when incremental steps of $\Gamma_{1}$ are applied to the reduced order nonlinear model. However, for the truth model, the effect of adding incremental steps at regular intervals is negligible on the output response, compared to the reduced order nonlinear model. We do not see transitions in the output behavior at time instants when incremental steps of $\Gamma_{1}$ are applied to the truth model, and the oscillations decay more uniformly in this case. 


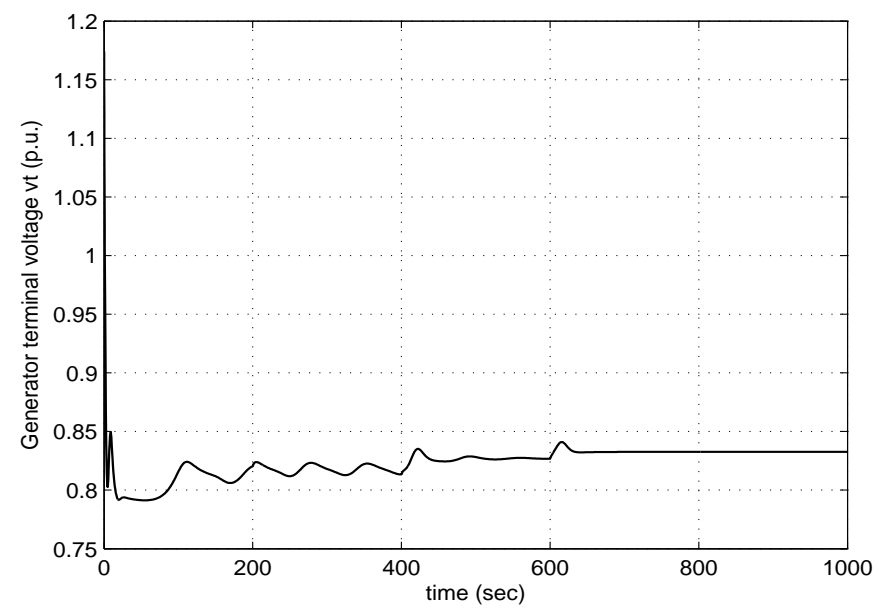

Figure 4.3: Plot of the generator terminal voltage $V_{t}$ vs time for $\Gamma_{1}$ (Test Signal 1) applied to the reduced order nonlinear model

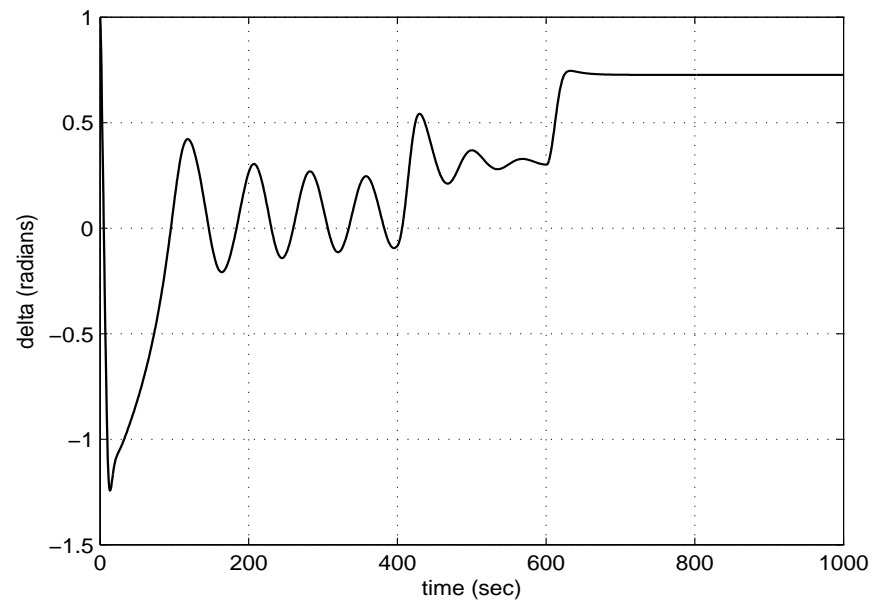

Figure 4.4: Plot of the rotor angle $\delta$ vs time for $\Gamma_{1}$ (Test Signal 1) applied to the reduced order nonlinear model

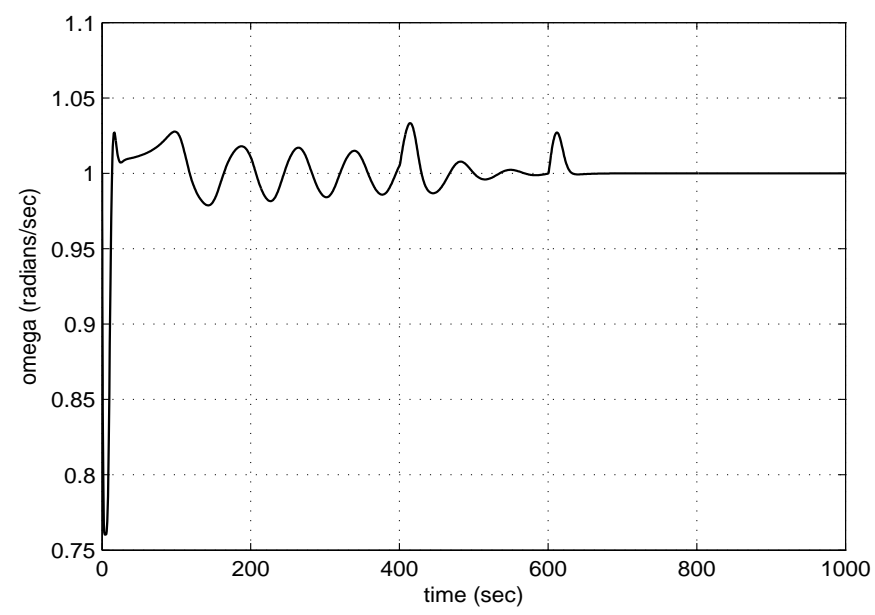

Figure 4.5: Plot of the frequency $\omega$ vs time for $\Gamma_{1}$ (Test Signal 1) applied to the reduced order nonlinear model 


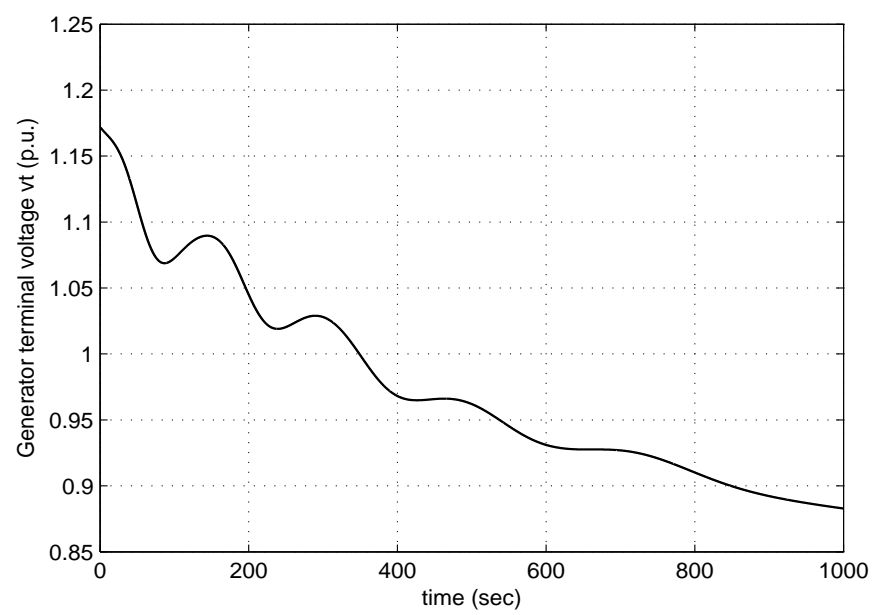

Figure 4.6: Plot of the generator terminal voltage $V_{t}$ vs time for $\Gamma_{1}$ (Test Signal 1) applied to the truth model

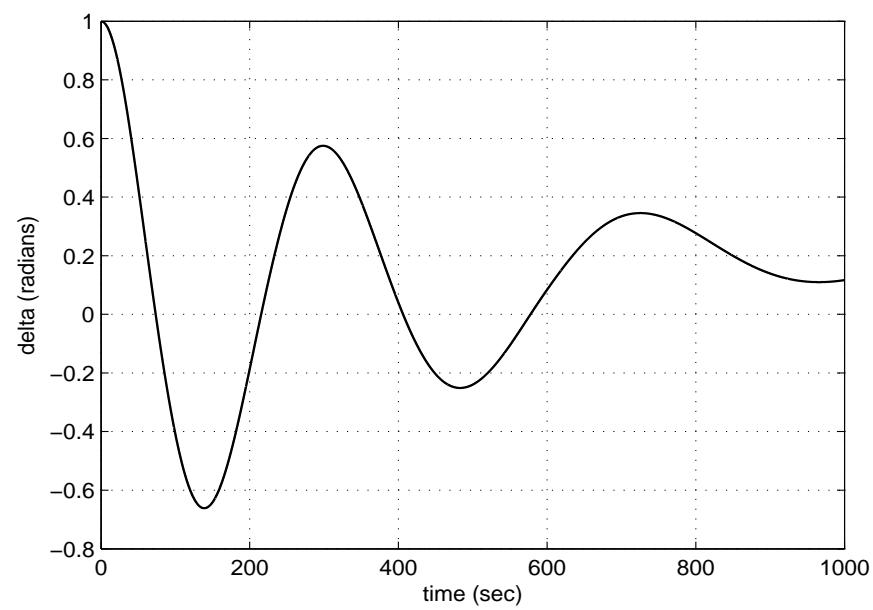

Figure 4.7: Plot of the rotor angle $\delta$ vs time for $\Gamma_{1}$ (Test Signal 1) applied to the truth model

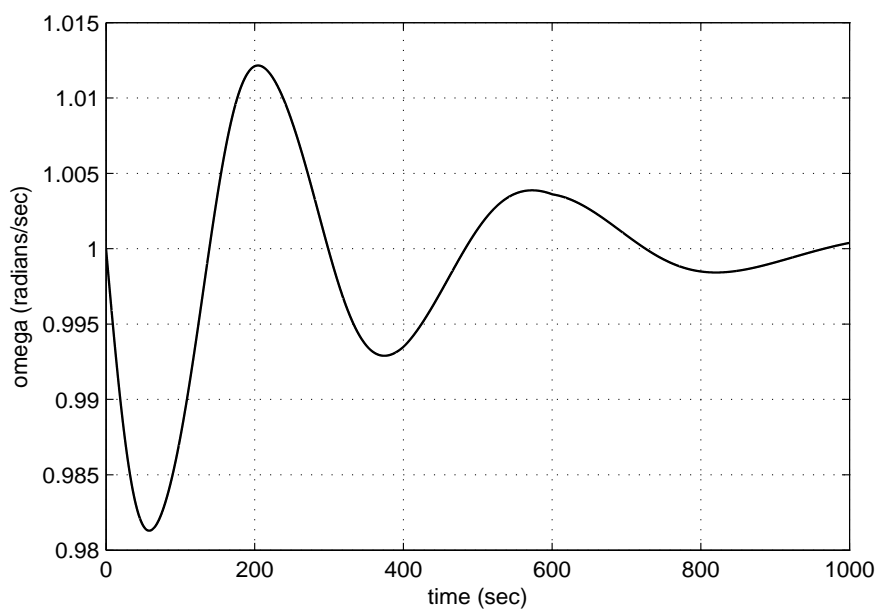

Figure 4.8: Plot of the angular velocity $\omega$ vs time for $\Gamma_{1}$ (Test Signal 1) applied to the truth model 
We now test the truth model and the reduced order nonlinear model of the SMIB for $\Gamma_{2}$ (Test signal 2) given in Figure 4.2. In this case the two inputs for the truth model are, $u=\left[V_{F}, u_{T}\right]^{\mathrm{T}}=\left[e_{15} \Gamma_{2}, \Gamma_{2}\right]^{\mathrm{T}}$, and the two inputs for the reduced order nonlinear model are, $u=\left[E_{F D}, u_{T}\right]^{\mathrm{T}}=\left[\Gamma_{2}, \Gamma_{2}\right]^{\mathrm{T}}$.

Figure 4.9, Figure 4.10, and Figure 4.11 show generator terminal voltage $V_{t}$, rotor angle $\delta$, and frequency $\omega$ vs time plots for $\Gamma_{2}$ (Test Signal 2) applied to the reduced order nonlinear model. From Figure 4.9 we can see that the generator terminal voltage $V_{t}$ settles to a new steady value of 0.82 p.u.. From Figure 4.10 we can see that the rotor angle $\delta$ first undergoes a large undershoot after which it oscillates about 0.1 p.u.. Figure 4.11 shows that the frequency $\omega$ oscillates about its steady state value of 1 p.u.. The oscillations decay with time. For $\Gamma_{2}$ (Test Signal 2) where the magnitudes of the incremental steps are reduced to half of $\Gamma_{1}$ (Test Signal 1), the effect of adding incremental steps of $\Gamma_{2}$ after regular intervals to the reduced order nonlinear model, is not as significant as compared to the effect of $\Gamma_{1}$ (Test Signal 1). The oscillations decay uniformly with time, and we do not see the sharp transitions that we saw when $\Gamma_{1}$ was applied to the reduced order nonlinear model.

Figure 4.12, Figure 4.13, and Figure 4.14 show generator terminal voltage $V_{t}$, rotor angle $\delta$, and frequency $\omega$ vs time plots for $\Gamma_{2}$ (Test Signal 2) applied to the truth model. These results are similar to the results obtained when $\Gamma_{1}$ (Test Signal 1) is applied to the truth model. 


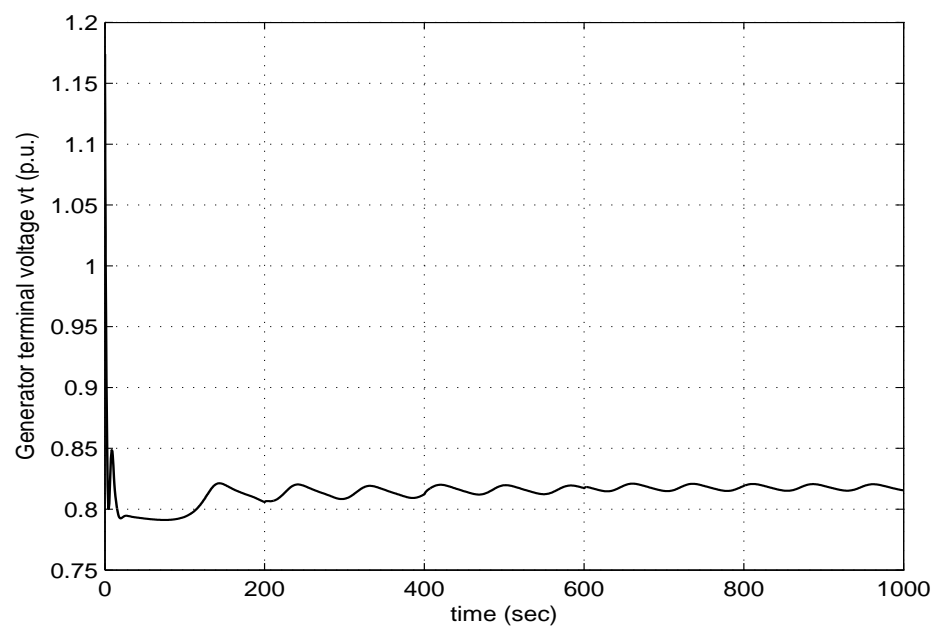

Figure 4.9: Plot of the generator terminal voltage $V_{t}$ vs time for $\Gamma_{2}$ (Test Signal 2) applied to the reduced order nonlinear model

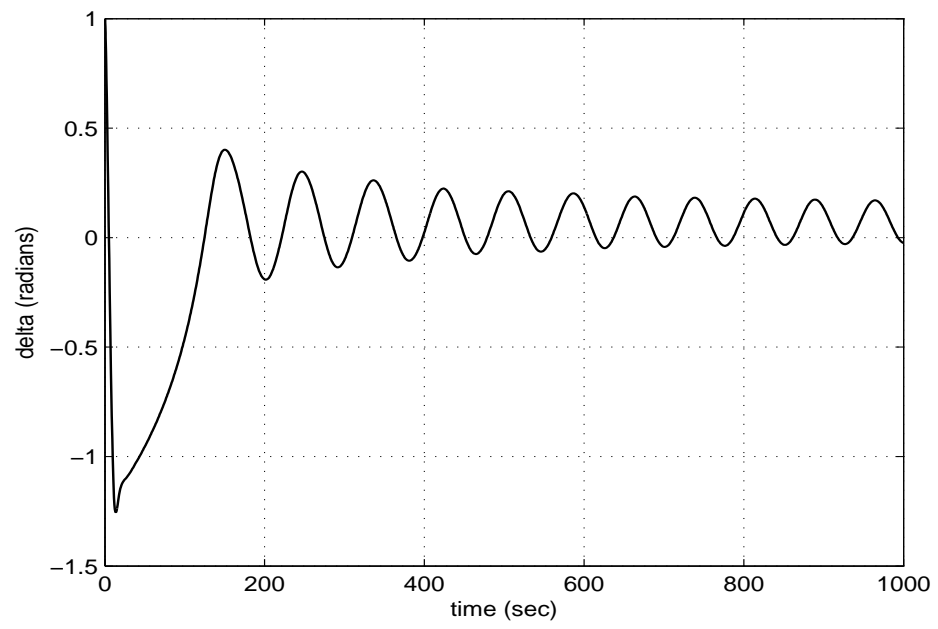

Figure 4.10: Plot of the rotor angle $\delta$ vs time for $\Gamma_{2}$ (Test Signal 2) applied to the reduced order nonlinear model

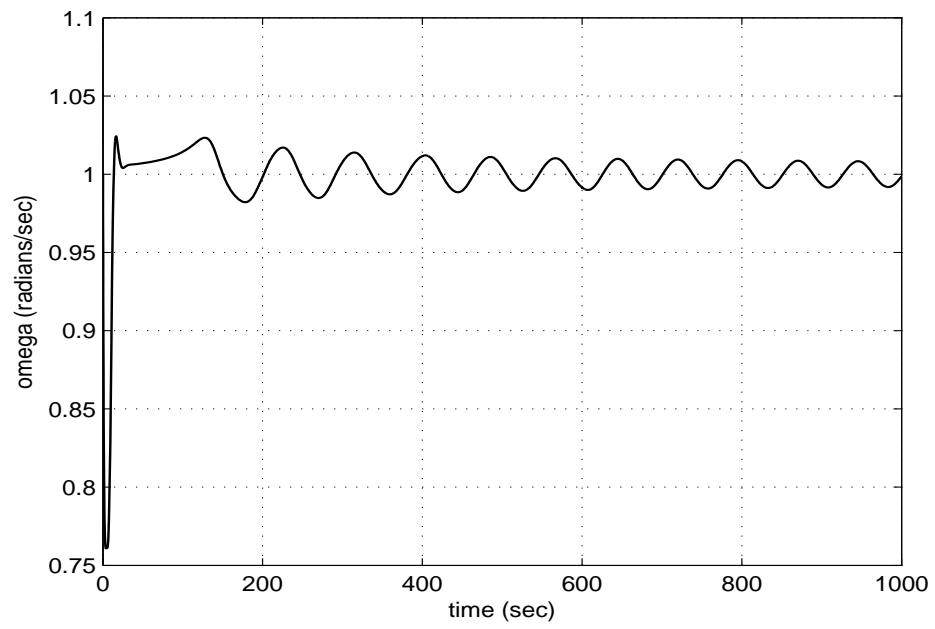

Figure 4.11: Plot of the frequency $\omega$ vs time for $\Gamma_{2}$ (Test Signal 2) applied to the reduced order nonlinear model 


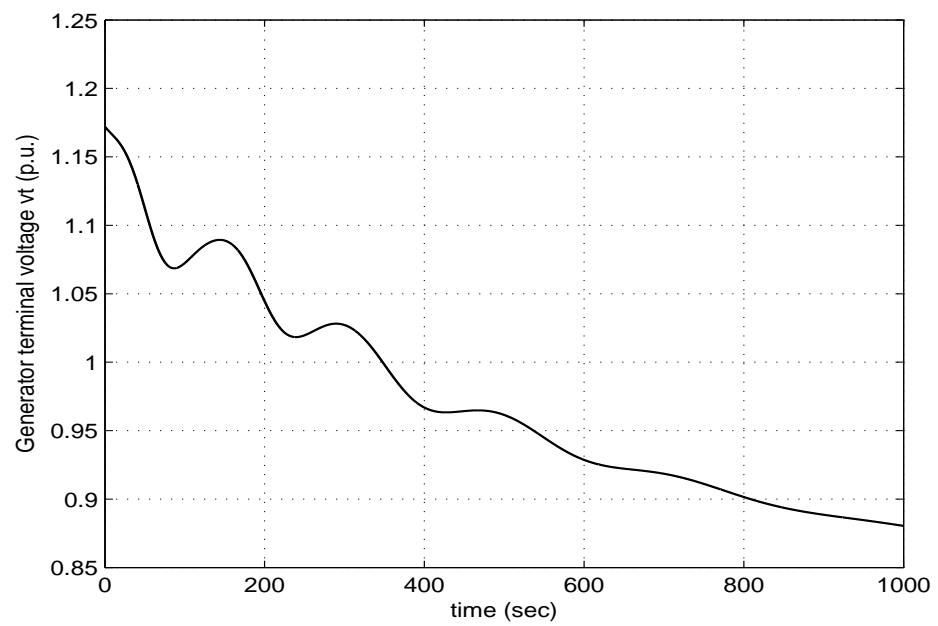

Figure 4.12: Plot of the generator terminal voltage $V_{t}$ vs time for $\Gamma_{2}$ (Test Signal 2) applied to the truth model

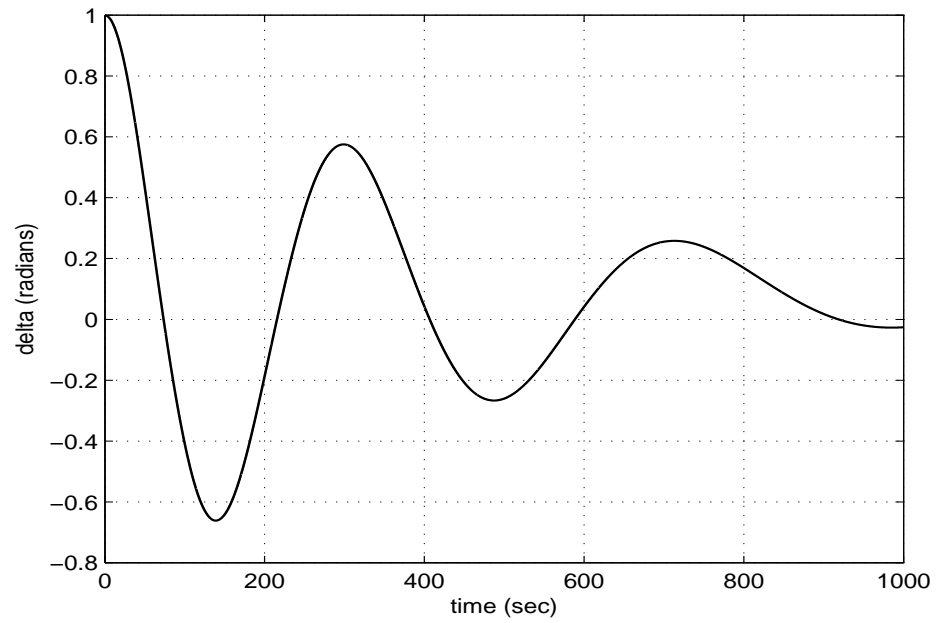

Figure 4.13: Plot of the rotor angle $\delta$ vs time for $\Gamma_{2}$ (Test Signal 2) applied to the truth model

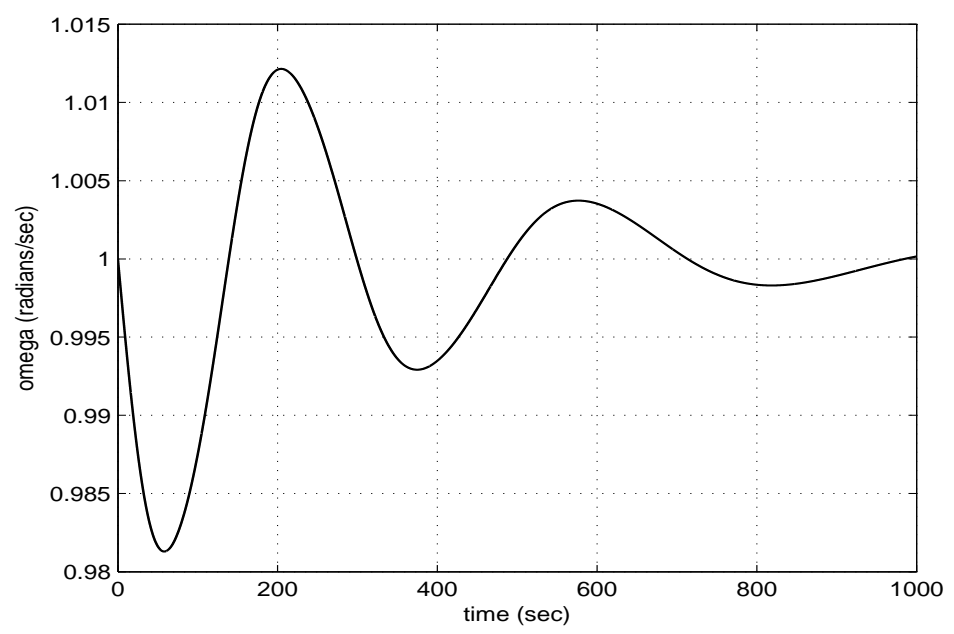

Figure 4.14: Plot of the angular velocity $\omega$ vs time for $\Gamma_{2}$ (Test Signal 2) applied to the truth model 


\section{The Decoupled Reduced Order Model}

Let us recall from the first section that small changes in real power are mainly dependent on changes in rotor angle $\delta$, and thus the frequency (i.e. on the prime mover valve control), whereas the reactive power is mainly dependent on the voltage magnitude (i.e. on the generator excitation). The excitation system time constant is much smaller than the prime mover time constant and its transient decay much faster and does not affect the load frequency control (LFC) dynamics. Thus, the cross-coupling between the LFC loop and the automatic voltage regulator (AVR) loop is negligible. Hence, load frequency control and excitation voltage control can be analyzed independently. Therefore, we decouple the two-input two-output (MIMO) reduced order system into two single input single output (SISO) subsystems. The first SISO subsystem consists of the LFC loop, where the turbine valve control $u_{T}$ controls the rotor angle $\delta$ and thus the frequency $\omega$ of the synchronous generator. The second SISO subsystem consists of the AVR loop, where the generator excitation $E_{F D}$ controls the terminal voltage $V_{t}$ of the synchronous generator. From Equation (3.59) and Equation (3.60) the linear model of the synchronous generator and turbine connected to an infinite bus is

$$
\begin{aligned}
& \Delta \dot{E}_{q}^{\prime}=f_{11} \Delta E_{q}^{\prime}+\left.\frac{\partial f_{1}}{\partial x_{3}}\right|_{x_{0}} \Delta \delta+g_{11} \Delta E_{F D} \\
& \Delta \dot{\omega}=\left.\frac{\partial f_{2}}{\partial x_{1}}\right|_{x_{0}} \Delta E_{q}^{\prime}+f_{27} \Delta \omega+\left.\frac{\partial f_{2}}{\partial x_{3}}\right|_{x_{0}} \Delta \delta+f_{28} \Delta T_{m} \\
& \Delta \dot{\delta}=\Delta \omega \\
& \Delta \dot{T}_{m}=f_{41} \Delta T_{m}+f_{42} \Delta G_{V} \\
& \Delta \dot{G}_{V}=f_{51} \Delta \omega+f_{52} \Delta G_{V}+g_{55} \Delta u_{T}
\end{aligned}
$$

Note that all the coefficients in the above expression are evaluated at the nominal operating point given in the previous section. Let us denote

$$
\begin{aligned}
& \left.\frac{\partial f_{1}}{\partial x_{3}}\right|_{x_{0}}=A_{13} \\
& \left.\frac{\partial f_{2}}{\partial x_{1}}\right|_{x_{0}}=A_{21} \\
& \left.\frac{\partial f_{2}}{\partial x_{3}}\right|_{x_{0}}=A_{23}
\end{aligned}
$$

The MIMO system with control inputs $\Delta E_{F D}$ and $\Delta u_{T}$ can be decoupled into the LFC loop with control input $\Delta u_{T}$ as

$$
\begin{aligned}
& \Delta \dot{\omega}=A_{21} \Delta E_{q}^{\prime}+f_{27} \Delta \omega+A_{23} \Delta \delta+f_{28} \Delta T_{m} \\
& \Delta \dot{\delta}=\Delta \omega \\
& \Delta \dot{T}_{m}=f_{41} \Delta T_{m}+f_{42} \Delta G_{v} \\
& \Delta \dot{G}_{V}=f_{51} \Delta \omega+f_{52} \Delta G_{V}+g_{55} \Delta u_{T}
\end{aligned}
$$

and the AVR loop with control input $\Delta E_{F D}$ as

$$
\Delta \dot{E}_{q}^{\prime}=f_{11} \Delta E_{q}^{\prime}+A_{13} \Delta \delta+g_{11} \Delta E_{F D}
$$

The weak coupling $A_{21} \Delta E_{q}^{\prime}$ between the LFC and the AVR loop can be neglected in the LFC loop in Equation (5.3), and the weak coupling $A_{13} \Delta \delta$ between the LFC and the AVR loop can be neglected in the AVR loop in Equation (5.4). Neglecting theses terms, the LFC loop with control input $\Delta u_{T}$ is approximated as

$$
\begin{aligned}
& \Delta \dot{\omega}=f_{27} \Delta \omega+A_{23} \Delta \delta+f_{28} \Delta T_{m} \\
& \Delta \dot{\delta}=\Delta \omega \\
& \Delta \dot{T}_{m}=f_{41} \Delta T_{m}+f_{42} \Delta G_{v} \\
& \Delta \dot{G}_{V}=f_{51} \Delta \omega+f_{52} \Delta G_{V}+g_{55} \Delta u_{T}
\end{aligned}
$$


and the AVR loop with control input $\Delta E_{F D}$ is approximated as

$$
\Delta \dot{E}_{q}^{\prime}=f_{11} \Delta E_{q}^{\prime}+g_{11} \Delta E_{F D}
$$

\section{$5.1 \quad$ LFC Dynamics}

In this section we present the dynamics of the (LFC) SISO system with control input $\Delta u_{T}$. We then perform root locus analysis of the uncompensated (LFC) SISO system. A PID controller is designed next to stabilize the (LFC) SISO system which is originally unstable. From Equation (5.5) the LFC dynamics are

$$
\begin{aligned}
& \Delta \dot{\omega}=f_{27} \Delta \omega+A_{23} \Delta \delta+f_{28} \Delta T_{m} \\
& \Delta \dot{\delta}=\Delta \omega \\
& \Delta \dot{T}_{m}=f_{41} \Delta T_{m}+f_{42} \Delta G_{v} \\
& \Delta \dot{G}_{V}=f_{51} \Delta \omega+f_{52} \Delta G_{V}+g_{55} \Delta u_{T}
\end{aligned}
$$

Differentiating $\Delta \dot{\omega}$ and substituting $\Delta \dot{\delta}=\Delta \omega$ we have

$$
\Delta \ddot{\omega}=f_{27} \Delta \dot{\omega}+A_{23} \Delta \omega+f_{28} \Delta \dot{T}_{m}
$$

Taking the Laplace transform of Equation (5.8) with zero initial conditions we get

$$
s^{2} \Delta \omega(s)=f_{27} s \Delta \omega(s)+A_{23} \Delta \omega(s)+f_{28} s \Delta T_{m}(s)
$$

On rearranging Equation (5.9) and taking the Laplace transform of $\Delta \dot{\delta}=\Delta \omega$

$$
\begin{aligned}
\frac{\Delta \omega(s)}{\Delta T_{m}(s)} & =\frac{f_{28} s}{s^{2}-f_{27} s-A_{23}} \\
\frac{\Delta \delta(s)}{\Delta \omega(s)} & =\frac{1}{s}
\end{aligned}
$$

Equation (5.10) evaluated at the nominal operating point gives

$$
\begin{aligned}
\frac{\Delta \omega(s)}{\Delta T_{m}(s)} & =\frac{0.211 s}{s^{2}+0.3054} \\
\frac{\Delta \delta(s)}{\Delta \omega(s)} & =\frac{1}{s}
\end{aligned}
$$

The dynamics of the turbine from Equation (5.7) can be written as

$$
\Delta \dot{T}_{m}=f_{41} \Delta T_{m}+f_{42} \Delta G_{v}
$$

Taking the Laplace transform of Equation (5.12) with zero initial conditions

$$
s \Delta T_{m}(s)=f_{41} \Delta T_{m}(s)+f_{42} \Delta G_{V}(s)
$$

Rearranging and expressing Equation (5.13) as a transfer function

$$
\frac{\Delta T_{m}(s)}{\Delta G_{V}(s)}=\frac{f_{42}}{s-f_{41}}
$$

Equation (5.14) evaluated at the nominal operating point gives

$$
\frac{\Delta T_{m}(s)}{\Delta G_{V}(s)}=\frac{2}{s+2}
$$




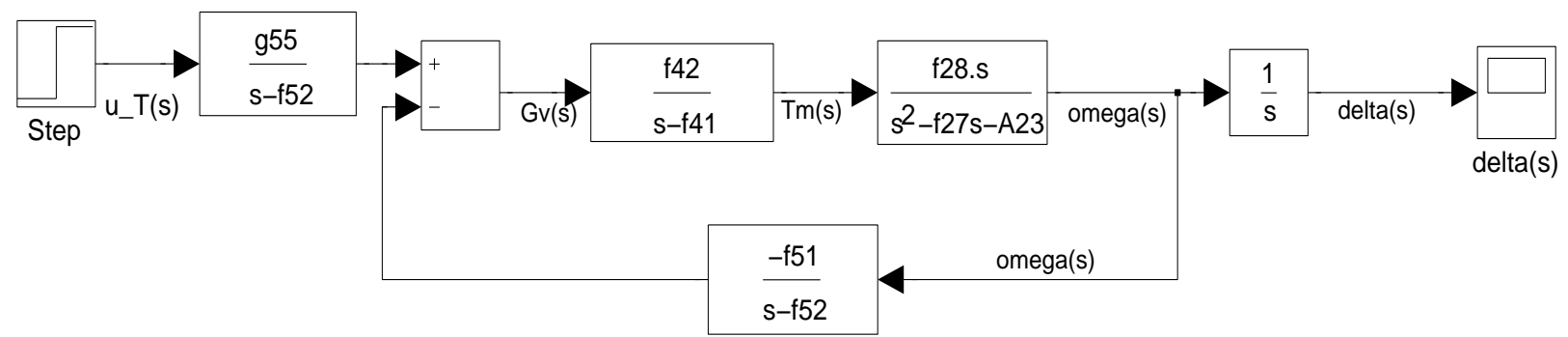

Figure 5.1: Simulink model of the (LFC) SISO system for a step input

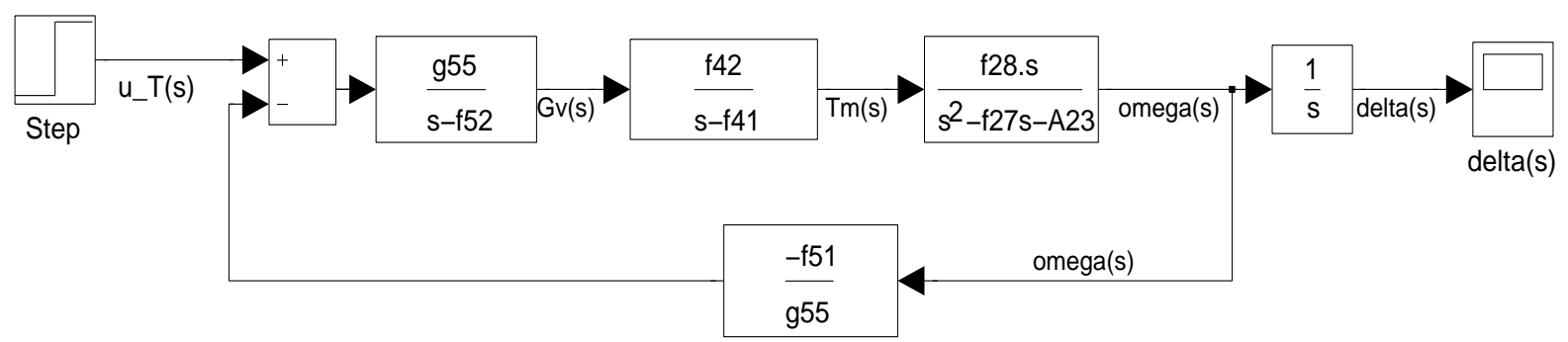

Figure 5.2: Simulink model of the (LFC) SISO system for a step input

From Equation (5.7) the governor dynamics can be written as

$$
\Delta \dot{G}_{V}=f_{51} \Delta \omega+f_{52} \Delta G_{V}+g_{55} \Delta u_{T}
$$

Taking the Laplace transform of Equation (5.16) with zero initial conditions

$$
s \Delta G_{V}(s)=f_{51} \Delta \omega(s)+f_{52} \Delta G_{V}(s)+g_{55} \Delta u_{T}(s)
$$

Rearranging and expressing Equation (5.17) as a transfer function

$$
\Delta G_{V}(s)=\frac{f_{51}}{s-f_{52}} \Delta \omega(s)+\frac{g_{55}}{s-f_{52}} \Delta u_{T}(s)
$$

Equation (5.18) evaluated at the nominal operating point gives

$$
\Delta G_{V}(s)=\frac{-0.25}{s+5} \Delta \omega(s)+\frac{5}{s+5} \Delta u_{T}(s)
$$

Figure 5.1 shows the simulink block diagram of the LFC loop for a step input. In all simulink models the subscript $\Delta$ is omitted for convenience. By moving the summing point ahead of the block $\frac{g_{55}}{s-f_{52}}$, the simulink model in Figure 5.1 can be simplified to get the simulink model shown in Figure 5.2. In Figure 5.2 let

$$
\begin{aligned}
G(s) & =\left(\frac{g_{55}}{s-f_{52}}\right)\left(\frac{f_{42}}{s-f_{41}}\right)\left(\frac{f_{28} s}{s^{2}-f_{27} s-A_{23}}\right) \\
& =\frac{g_{55} f_{42} f_{28} s}{\left(s-f_{52}\right)\left(s-f_{41}\right)\left(s^{2}-f_{27} s-A_{23}\right)}
\end{aligned}
$$

and

$$
H(s)=-\frac{f_{51}}{g_{55}}
$$

Using Equation (5.20) and Equation (5.21), the simulink model in Figure 5.2 can be simplified to the simulink model shown in Figure 5.3. The open loop transfer function of the SISO system shown in Figure 5.3 with output $\omega(s)$ and step input $u_{T}(s)$ is

$$
G(s) H(s)=-\frac{f_{42} f_{28} f_{51} s}{\left(s-f_{52}\right)\left(s-f_{41}\right)\left(s^{2}-f_{27} s-A_{23}\right)}
$$




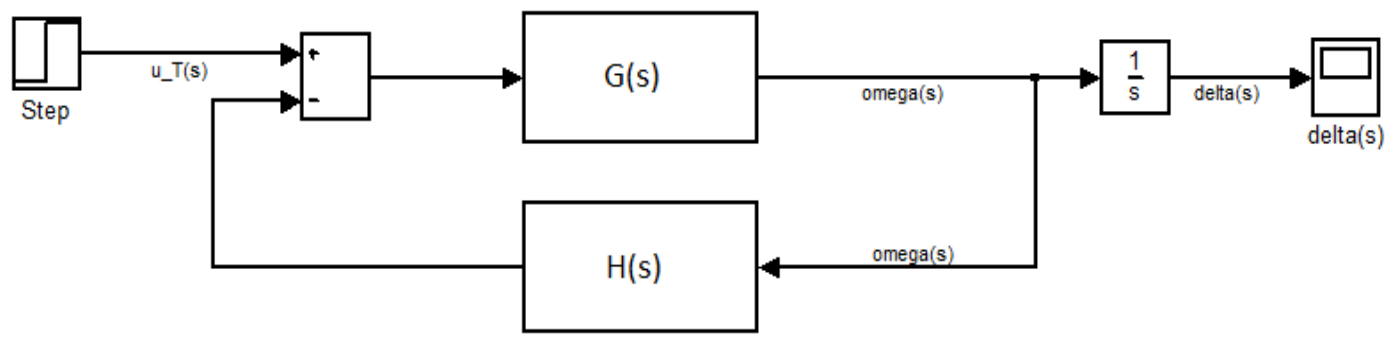

Figure 5.3: Simulink model of the (LFC) SISO system for a step input

The closed loop transfer function of the SISO system between the output $\Delta \delta(s)$ and control input $\Delta u_{T}(s)$ is

$$
\begin{aligned}
\frac{\Delta \delta(s)}{\Delta u_{T}(s)} & =\frac{G(s)}{1+G(s) H(s)}\left(\frac{1}{s}\right)=\frac{\frac{g_{55} f_{42} f_{28}}{\left(s-f_{52}\right)\left(s-f_{41}\right)\left(s^{2}-f_{27} s-A_{23}\right)}}{1-\frac{f_{42} f_{28} f_{51} s}{\left(s-f_{52}\right)\left(s-f_{41}\right)\left(s^{2}-f_{27} s-A_{23}\right)}} \\
& =\frac{g_{55} f_{42} f_{28}}{\left(s-f_{52}\right)\left(s-f_{41}\right)\left(s^{2}-f_{27} s-A_{23}\right)-f_{51} f_{42} f_{28} s}
\end{aligned}
$$

For a step input, $\Delta u_{T}(s)=\frac{1}{s}$. From the final value theorem, the steady state value of $\Delta \delta(s)$ is

$$
\begin{aligned}
\Delta \delta_{s s} & =\lim _{s \rightarrow 0} s \Delta \delta(s) \\
& =\lim _{s \rightarrow 0} s \Delta u_{T}(s) \frac{g_{55} f_{42} f_{28}}{\left(s-f_{52}\right)\left(s-f_{41}\right)\left(s^{2}-f_{27} s-A_{23}\right)-f_{51} f_{42} f_{28} s} \\
& =-\frac{g_{55} f_{42} f_{28}}{f_{52} f_{41} A_{23}}
\end{aligned}
$$

Substituting numerical values for the constant parameters in Equation (5.24) we get

$$
\Delta \delta_{s s}=0.6909
$$

Also

$$
\begin{aligned}
\frac{\Delta \omega(s)}{\Delta u_{T}(s)} & =\frac{G(s)}{1+G(s) H(s)}=\frac{\frac{g_{55} f_{42} f_{28} s}{\left(s-f_{52}\right)\left(s-f_{41}\right)\left(s^{2}-f_{27} s-A_{23}\right)}}{1-\frac{f_{42} f_{28} f_{51} s}{\left(s-f_{52}\right)\left(s-f_{41}\right)\left(s^{2}-f_{27} s-A_{23}\right)}} \\
& =\frac{g_{55} f_{42} f_{28} s}{\left(s-f_{52}\right)\left(s-f_{41}\right)\left(s^{2}-f_{27} s-A_{23}\right)-f_{51} f_{42} f_{28} s}
\end{aligned}
$$

From the final value theorem, the steady state value of $\Delta \omega(s)$ for a step input is

$$
\begin{aligned}
\Delta \omega_{s s} & =\lim _{s \rightarrow 0} s \Delta \omega(s) \\
& =\lim _{s \rightarrow 0} s \Delta u_{T}(s) \frac{g_{55} f_{42} f_{28} s}{\left(s-f_{52}\right)\left(s-f_{41}\right)\left(s^{2}-f_{27} s-A_{23}\right)-f_{51} f_{42} f_{28} s} \\
& =-\lim _{s \rightarrow 0} \frac{g_{55} f_{42} f_{28}}{f_{52} f_{41} A_{23}} s \\
& =0
\end{aligned}
$$

Figure 5.4 and Figure 5.5 show the plots of $\Delta \delta(\mathrm{s})$ and $\Delta \omega(\mathrm{s})$ vs time for the uncompensated (LFC) SISO system for a step input, respectively. From these plots we can see that $\Delta \delta(\mathrm{s})$ settles to a steady state value of 0.6909 and $\Delta \omega(\mathrm{s})$ settles to a steady state value of 0 which are in agreement with the respective steady state values calculated in Equation (5.25) and Equation (5.27). Also the root locus plot in Figure 5.6 clearly shows that the uncompensated (LFC) SISO system with step input $u_{T}(s)$ is unstable. 


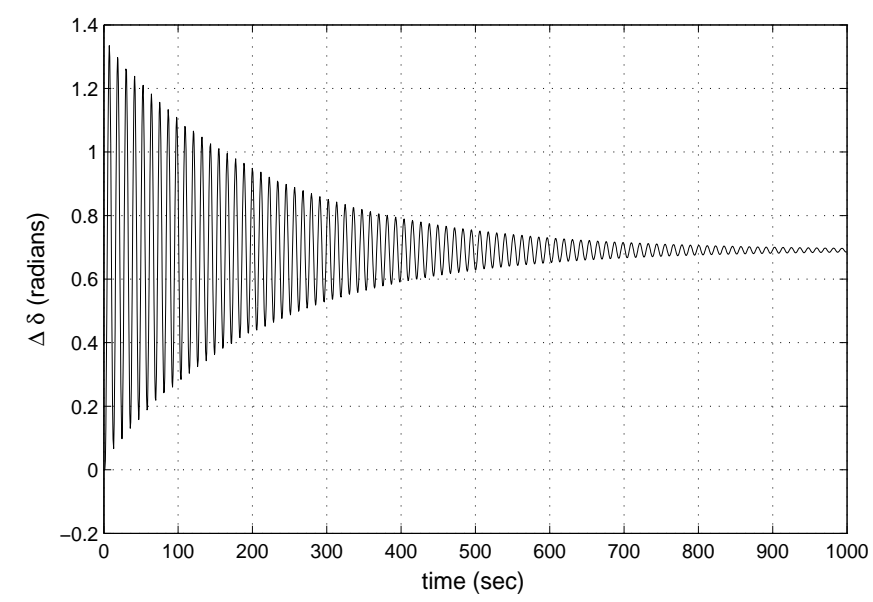

Figure 5.4: Plot of $\Delta \delta(\mathrm{s})$ vs time for the uncompensated (LFC) SISO system for a step input

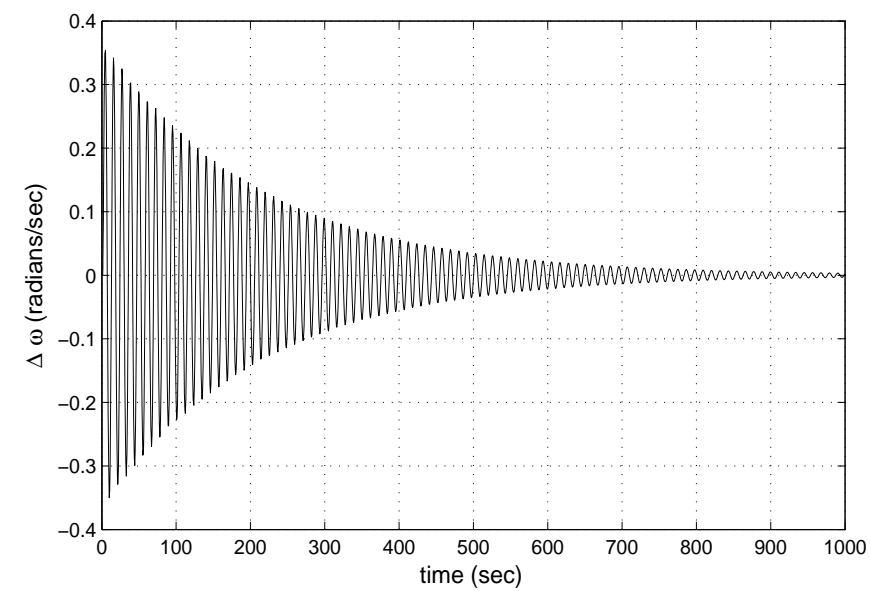

Figure 5.5: Plot of $\Delta \omega(\mathrm{s})$ vs time for the uncompensated (LFC) SISO system for a step input

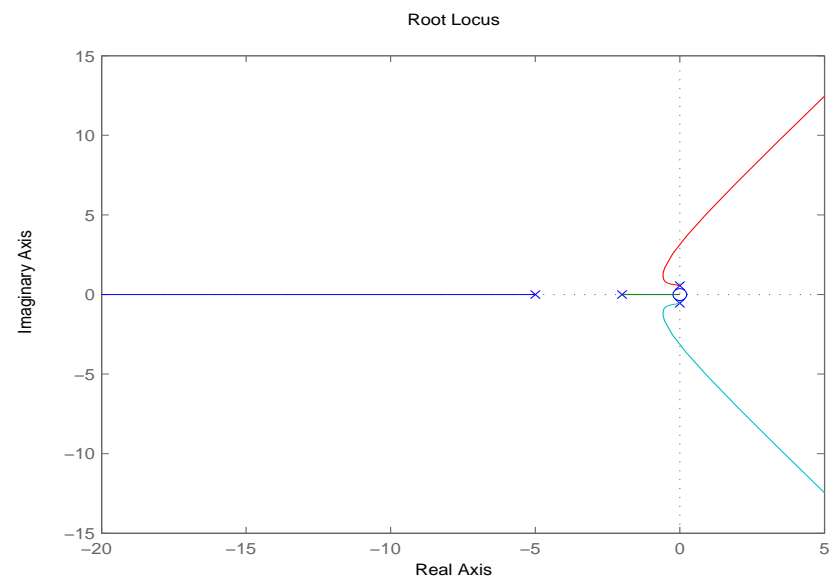

Figure 5.6: Root locus plot for the uncompensated (LFC) SISO system 


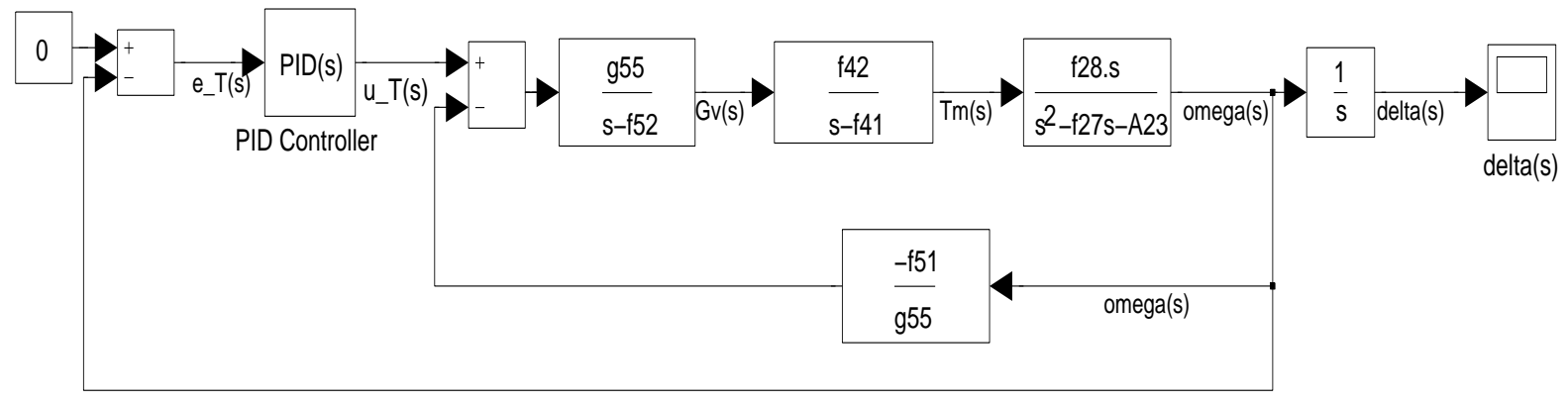

Figure 5.7: Simulink model of the PID compensated (LFC) SISO system

\subsubsection{PID controller design for the LFC loop}

In order to stabilize this system we design a proportional-integral-derivative (PID) controller. The simulink model of the PID compensated (LFC) SISO system is given in Figure 5.7. The output of the PID controller $\Delta u_{T}(s)$ in the frequency domain is given by

$$
\Delta u_{T}(s)=K_{p 1} \Delta e_{T}(s)+\frac{K_{i 1}}{s} \Delta e_{T}(s)+K_{d 1} s \Delta e_{T}(s)
$$

where $K_{p 1}$ is the proportional gain, $K_{i 1}$ is the integral gain, $K_{d 1}$ is the derivative gain, and $\Delta e_{T}(s)$ is the error signal. Expressing Equation (5.28) as a transfer function

$$
\begin{aligned}
\frac{\Delta u_{T}(s)}{\Delta e_{T}(s)} & =K_{p 1}+\frac{K_{i 1}}{s}+K_{d 1} s \\
& =\frac{K_{d 1} s^{2}+K_{p 1} s+K_{i 1}}{s}
\end{aligned}
$$

From Figure 5.7 the open loop transfer function of the PID compensated (LFC) SISO system can be written as

$$
\begin{aligned}
G_{P I D 1}(s) & =\left(\frac{K_{d 1} s^{2}+K_{p 1} s+K_{i 1}}{s}\right) \frac{G(s)}{1+G(s) H(s)} \\
& =\left(\frac{K_{d 1} s^{2}+K_{p 1} s+K_{i 1}}{s}\right)\left(\frac{g_{55} f_{42} f_{28} s}{\left(s-f_{52}\right)\left(s-f_{41}\right)\left(s^{2}-f_{27} s-A_{23}\right)-f_{51} f_{42} f_{28} s}\right) \\
H_{P I D 1}(s) & =1 \\
\therefore G_{P I D 1}(s) H_{P I D 1}(s) & =\left(\frac{K_{d 1} s^{2}+K_{p 1} s+K_{i 1}}{s}\right)\left(\frac{g_{55} f_{42} f_{28} s}{\left(s-f_{52}\right)\left(s-f_{41}\right)\left(s^{2}-f_{27} s-A_{23}\right)-f_{51} f_{42} f_{28} s}\right)
\end{aligned}
$$

The PID gains are tuned to $K_{p 1}=200, K_{i 1}=150$, and $K_{d 1}=100$. Substituting these PID gains and numerical values for the constant coefficients in Equation (5.30) we get

$$
G_{P I D 1}(s) H_{P I D 1}(s)=\frac{52.74 s^{3}+105.5 s^{2}+79.11 s}{5 s^{5}+35 s^{4}+51.53 s^{3}+10.69 s^{2}+15.27 s}
$$

Figure 5.8 and Figure 5.9 show the plots for $\Delta \delta(\mathrm{s})$ and $\Delta \omega(\mathrm{s})$ vs time for the PID compensated (LFC) SISO system, respectively. Also, Figure 5.10 shows the root locus plot for the PID compensated (LFC) SISO system. From this plot it is evident that the PID compensated (LFC) SISO system is stable since the root locus lies entirely in the left half s-plane. 


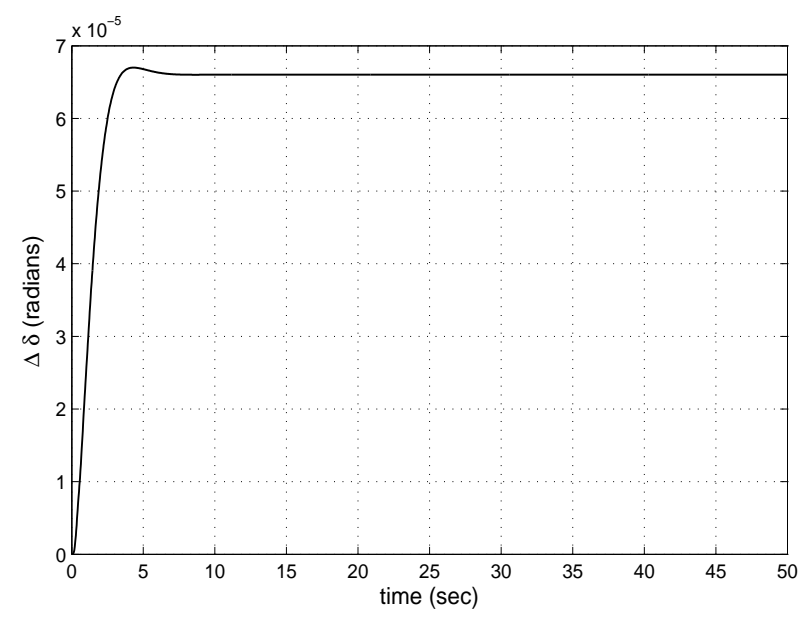

Figure 5.8: Plot of $\Delta \delta(\mathrm{s})$ vs time for the PID compensated (LFC) SISO system

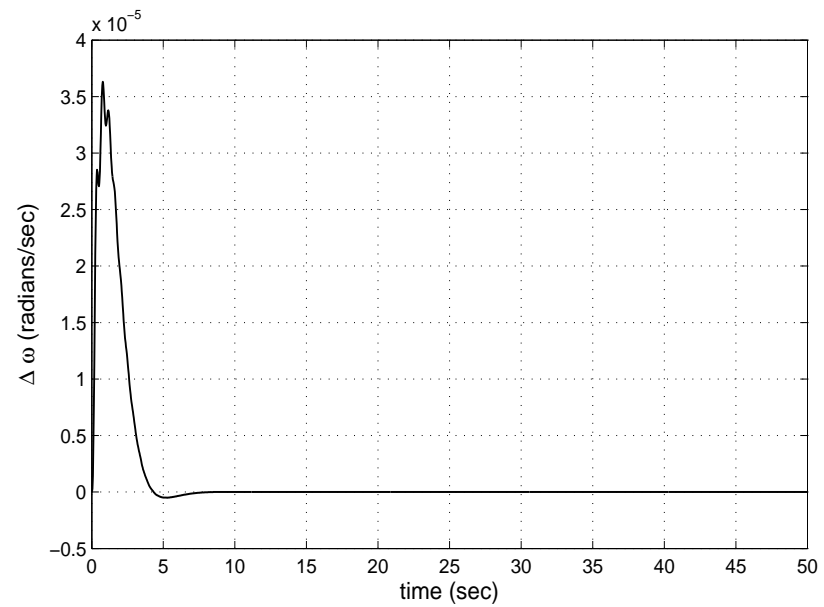

Figure 5.9: Plot of $\Delta \omega(\mathrm{s})$ vs time for the PID compensated (LFC) SISO system

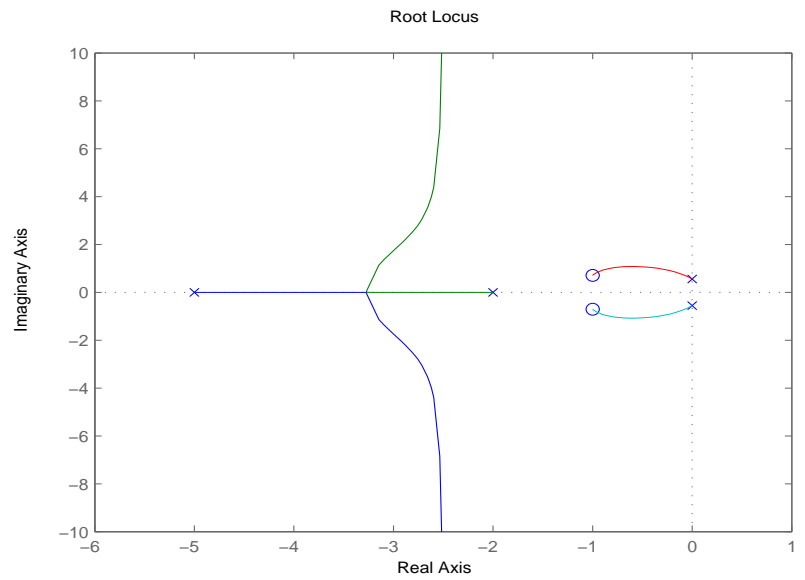

Figure 5.10: Root locus plot for the PID compensated (LFC) SISO system 


\section{$5.2 \quad$ AVR Dynamics}

In this section we present the dynamics of the (AVR) SISO system with control input $\Delta E_{F D}$. In addition we also design a PID controller for the (AVR) SISO system. From Equation (5.6) the dynamics of the (AVR) SISO system are

$$
\Delta \dot{E}_{q}^{\prime}=f_{11} \Delta E_{q}^{\prime}+g_{11} \Delta E_{F D}
$$

Taking the Laplace transform of Equation (5.32) assuming zero initial conditions we get

$$
s \Delta E_{q}^{\prime}(s)=f_{11} \Delta E_{q}^{\prime}(s)+g_{11} \Delta E_{F D}(s)
$$

Rearranging and expressing Equation (5.33) as a transfer function

$$
\frac{\Delta E_{q}^{\prime}(s)}{\Delta E_{F D}(s)}=\frac{g_{11}}{s-f_{11}}
$$

From Equation (3.71) the output terminal voltage $\Delta V_{t}$ is

$$
\Delta V_{t}=T_{1} \Delta E_{q}^{\prime}+T_{2} \Delta \delta
$$

For the decoupled (AVR) SISO system with $\Delta E_{F D}$ as the input and $\Delta V_{t}$ as the output the coupling $T_{2} \Delta \delta$ between the (LFC) SISO system and the (AVR) SISO system is neglected. Therefore,

$$
\Delta V_{t}=T_{1} \Delta E_{q}^{\prime}
$$

Taking the Laplace transform of Equation (5.36)

$$
\Delta V_{t}(s)=T_{1} \Delta E_{q}^{\prime}(s)
$$

Combining Equation (5.34) and Equation (5.37) we get

$$
\frac{\Delta V_{t}(s)}{\Delta E_{F D}(s)}=\frac{T_{1} g_{11}}{s-f_{11}}
$$

Substituting numerical values for the coefficients in Equation (5.38) evaluated at the nominal operating point we get

$$
\frac{\Delta V_{t}(s)}{\Delta E_{F D}(s)}=\frac{0.08913}{s+0.5517}
$$

Figure 5.11 shows the simulink model of the uncompensated (AVR) SISO system for a step input. In Figure 5.11 let

$$
G_{1}(s)=\frac{T_{1} g_{11}}{s-f_{11}}
$$

and

$$
H_{1}(s)=1
$$

Therefore, the open loop transfer function of the uncompensated (AVR) SISO system shown in Figure 5.11 is

$$
G_{1}(s) H_{1}(s)=\frac{T_{1} g_{11}}{s-f_{11}}
$$

The closed loop transfer function of the uncompensated (AVR) SISO system shown in Figure 5.11 is

$$
\begin{aligned}
\frac{\Delta V_{t}(s)}{\Delta E_{r e f}(s)} & =\frac{G_{1}(s)}{1+G_{1}(s) H_{1}(s)}=\frac{\frac{T_{1} g_{11}}{s-f_{11}}}{1+\frac{T_{1} g_{11}}{s-f_{11}}} \\
& =\frac{T_{1} g_{11}}{s-f_{11}+T_{1} g_{11}}
\end{aligned}
$$




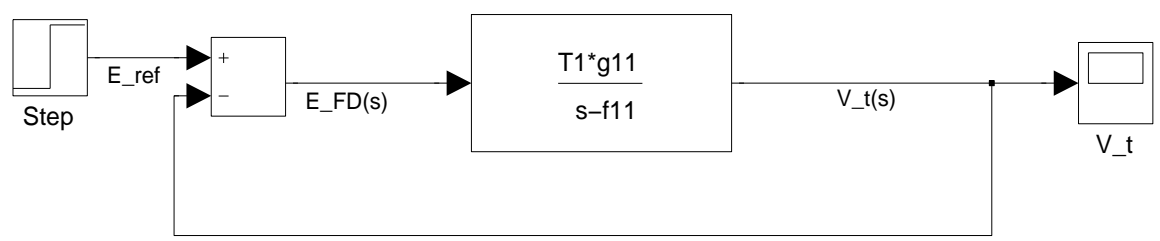

Figure 5.11: Simulink model of the uncompensated (AVR) SISO system for a step input

For a step input, $\Delta E_{r e f}(s)=\frac{1}{s}$. From the final value theorem, the steady state value of $\Delta V_{t}(s)$ is

$$
\begin{aligned}
\Delta V_{t(s s)} & =\lim _{s \rightarrow 0} s \Delta V_{t}(s) \\
& =\lim _{s \rightarrow 0} s \Delta E_{r e f}(s)\left(\frac{T_{1} g_{11}}{s-f_{11}+T_{1} g_{11}}\right) \\
& =\frac{T_{1} g_{11}}{T_{1} g_{11}-f_{11}}
\end{aligned}
$$

Substituting numerical values for the coefficients in Equation (5.44)

$$
\Delta V_{t(s s)}=0.1391
$$

Figure 5.12 shows the terminal voltage $\Delta V_{t}(\mathrm{~s})$ vs time plot for the uncompensated (AVR) SISO system for a step input. From this plot we can see that $\Delta V_{t}(\mathrm{~s})$ settles to a steady state value of 0.1391 which is in agreement with the steady state value calculated in Equation (5.45). Figure 5.13 shows the root locus plot for the uncompensated (AVR) SISO system. From this plot it is evident that the uncompensated (AVR) SISO system is stable. 


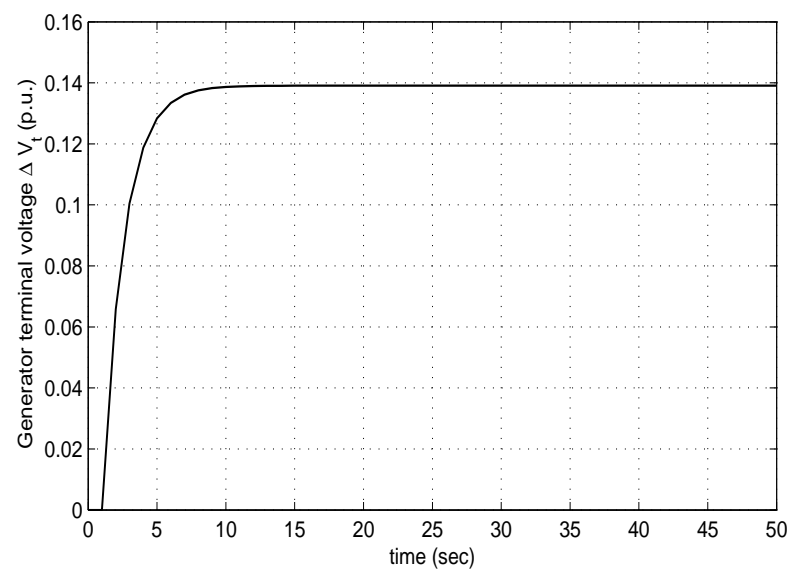

Figure 5.12: Plot of the terminal voltage $\Delta V_{t}(\mathrm{~s})$ vs time for the uncompensated (AVR) SISO system for a step input

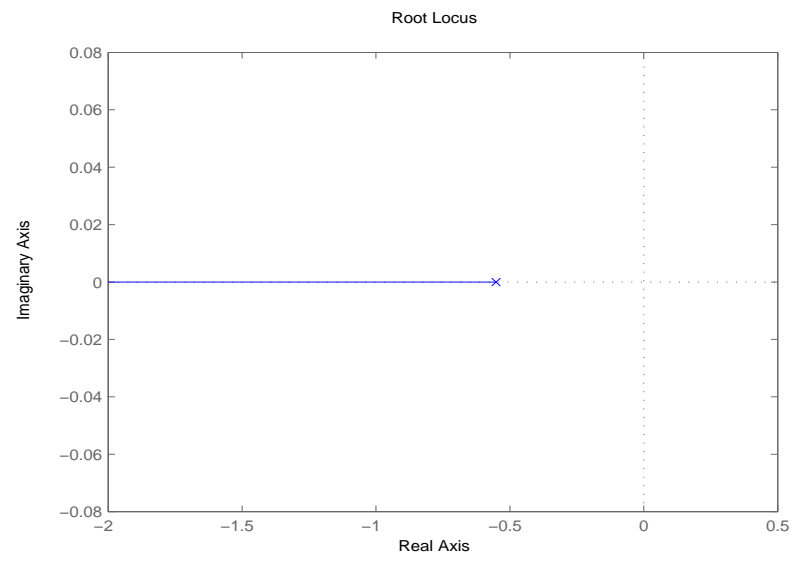

Figure 5.13: Root locus plot for the uncompensated (AVR) SISO system

\subsubsection{PID controller design for the AVR loop}

We design a PID controller to improve the transient and steady state response of the (AVR) SISO system. The output of this PID controller $\Delta E_{F D}(s)$ in the frequency domain is given by

$$
\Delta E_{F D}(s)=K_{p 2} \Delta e_{F D}(s)+\frac{K_{i 2}}{s} \Delta e_{F D}(s)+K_{d 2} s \Delta e_{F D}(s)
$$

where $K_{p 2}$ is the proportional gain, $K_{i 2}$ is the integral gain, $K_{d 2}$ is the derivative gain, and $\Delta e_{F D}(s)$ is the error signal. Expressing Equation (5.46) as a transfer function

$$
\begin{aligned}
\frac{\Delta E_{F D}(s)}{\Delta e_{F D}(s)} & =K_{p 2}+\frac{K_{i 2}}{s}+K_{d 2} s \\
& =\frac{K_{d 2} s^{2}+K_{p 2} s+K_{i 2}}{s}
\end{aligned}
$$




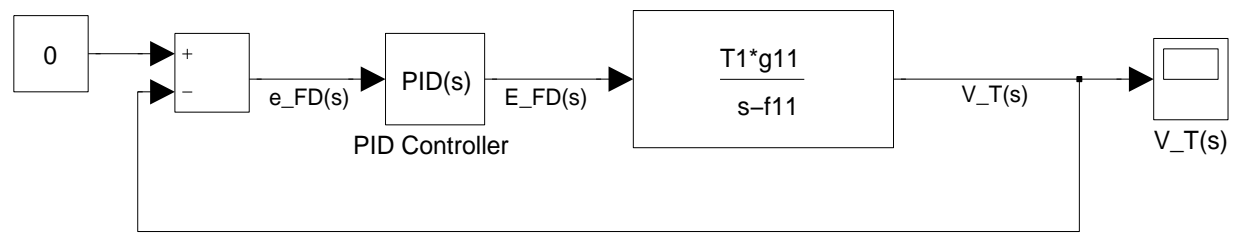

Figure 5.14: Simulink model of the PID compensated (AVR) SISO system

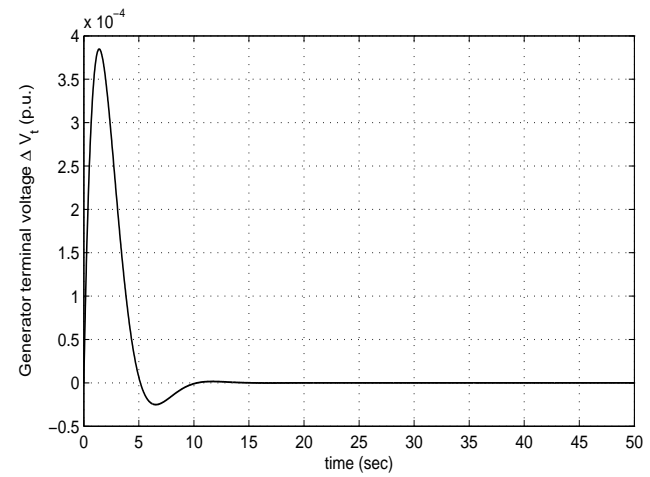

Figure 5.15: Plot of $\Delta V_{t}(\mathrm{~s})$ vs time for the PID compensated (AVR) SISO system

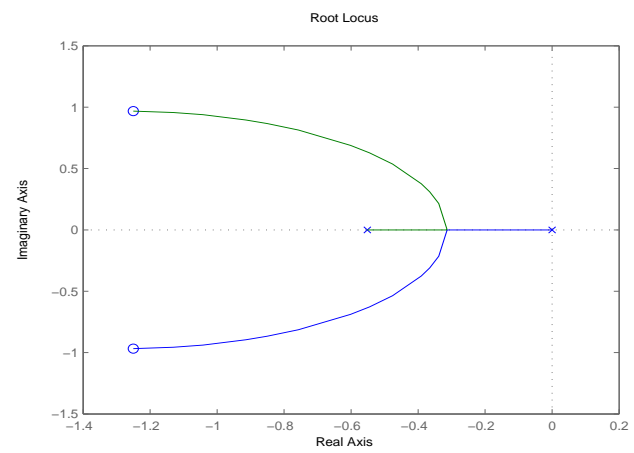

Figure 5.16: Root locus plot for the PID compensated (AVR) SISO system

From Figure 5.14 the open loop transfer function of the PID compensated (AVR) SISO system can be written as

$$
\begin{aligned}
G_{P I D 2}(s) & =\left(\frac{K_{d 2} s^{2}+K_{p 2} s+K_{i 2}}{s}\right)\left(\frac{T_{1} g_{11}}{s-f_{11}}\right) \\
H_{P I D 2}(s) & =1 \\
\therefore G_{P I D 2}(s) H_{P I D 2}(s) & =\left(\frac{K_{d 2} s^{2}+K_{p 2} s+K_{i 2}}{s}\right)\left(\frac{T_{1} g_{11}}{s-f_{11}}\right)
\end{aligned}
$$

The PID gains $K_{p 2}=10, K_{i 2}=10$, and $K_{d 2}=4$ are tuned so as to get the best system response. Substituting these PID gains and numerical values for the constant coefficients in Equation (5.48) we get

$$
G_{P I D 2}(s) H_{P I D 2}(s)=\frac{0.3565 s^{2}+0.8913 s+0.8913}{s^{2}+0.5517 s}
$$

Figure 5.15 shows the generator terminal voltage $\Delta V_{t}(\mathrm{~s})$ vs time plot for the PID compensated (AVR) SISO system. From this plot we can see that $\Delta V_{t}(\mathrm{~s})$ attains its steady state value of 0 in approximately 10 seconds. Figure 5.16 shows the root locus plot for the PID compensated (AVR) SISO system. The PID compensated (AVR) SISO system is stable since the root locus lies in the left half s-plane. 


\section{3 $\quad$ LFC and AVR including coupling}

As there is weak coupling between the LFC and AVR subsystems, the rotor angle $\delta$ and thus the frequency $\omega$, and the terminal voltage $V_{t}$ were controlled separately. In this section we study the effect of coupling between the LFC and the AVR system. In the previous section we saw that the weak coupling $A_{21} \Delta E_{q}^{\prime}$ between the LFC and the AVR loop which appeared in the coupled system in Equation (5.1) was neglected in the LFC loop in Equation (5.5). Also, the weak coupling $A_{13} \Delta \delta$ between the LFC and the AVR loop which appeared in the coupled system in Equation (5.1) was neglected in the AVR loop in Equation (5.6). In the coupled system including the LFC and AVR dynamics we do not neglect these terms. Thus, from Equation (5.1) we can write

$$
\begin{aligned}
& \Delta \dot{E}_{q}^{\prime}=f_{11} \Delta E_{q}^{\prime}+A_{13} \Delta \delta+g_{11} \Delta E_{F D} \\
& \Delta \dot{\omega}=A_{21} \Delta E_{q}^{\prime}+f_{27} \Delta \omega+A_{23} \Delta \delta+f_{28} \Delta T_{m} \\
& \Delta \dot{\delta}=\Delta \omega
\end{aligned}
$$

Taking the Laplace transform of the expression for $\Delta \dot{E}_{q}^{\prime}$ in Equation (5.50)

$$
s \Delta E_{q}^{\prime}(s)=f_{11} \Delta E_{q}^{\prime}(s)+A_{13} \Delta \delta(s)+g_{11} \Delta E_{F D}(s)
$$

Rearranging Equation (5.51) we get

$$
\Delta E_{q}^{\prime}(s)=\frac{A_{13}}{s-f_{11}} \Delta \delta(s)+\frac{g_{11}}{s-f_{11}} \Delta E_{F D}(s)
$$

Also from Equation (3.71) the first output which is the terminal voltage $V_{t}$ of the synchronous generator for the coupled system including LFC and AVR dynamics is

$$
\Delta V_{t}=T_{1} \Delta E_{q}^{\prime}+T_{2} \Delta \delta
$$

The Laplace transform of Equation (5.53) gives

$$
\Delta V_{t}(s)=T_{1} \Delta E_{q}^{\prime}(s)+T_{2} \Delta \delta(s)
$$

Next, taking the derivative of $\Delta \dot{\omega}$ we get

$$
\Delta \ddot{\omega}=A_{21} \Delta \dot{E}_{q}^{\prime}+f_{27} \Delta \dot{\omega}+A_{23} \Delta \omega+f_{28} \Delta \dot{T}_{m}
$$

Taking the Laplace transform of Equation (5.55)

$$
s^{2} \Delta \omega(s)=A_{21} s \Delta E_{q}^{\prime}(s)+f_{27} s \Delta \omega(s)+A_{23} \Delta \omega(s)+f_{28} s \Delta T_{m}(s)
$$

Rearranging Equation (5.56) we get

$$
\Delta \omega(s)=\frac{A_{21} s}{s^{2}-f_{27} s-A_{23}} \Delta E_{q}^{\prime}(s)+\frac{f_{28} s}{s^{2}-f_{27} s-A_{23}} \Delta T_{m}(s)
$$

Also the Laplace transform of $\Delta \dot{\delta}=\Delta \omega$ is $s \Delta \delta(s)=\Delta \omega(s)$. Thus, the second output which is the rotor angle $\Delta \delta(s)$ of the synchronous generator for the coupled system including the LFC and AVR dynamics can be written as

$$
\Delta \delta(s)=\frac{A_{21}}{s^{2}-f_{27} s-A_{23}} \Delta E_{q}^{\prime}(s)+\frac{f_{28}}{s^{2}-f_{27} s-A_{23}} \Delta T_{m}(s)
$$




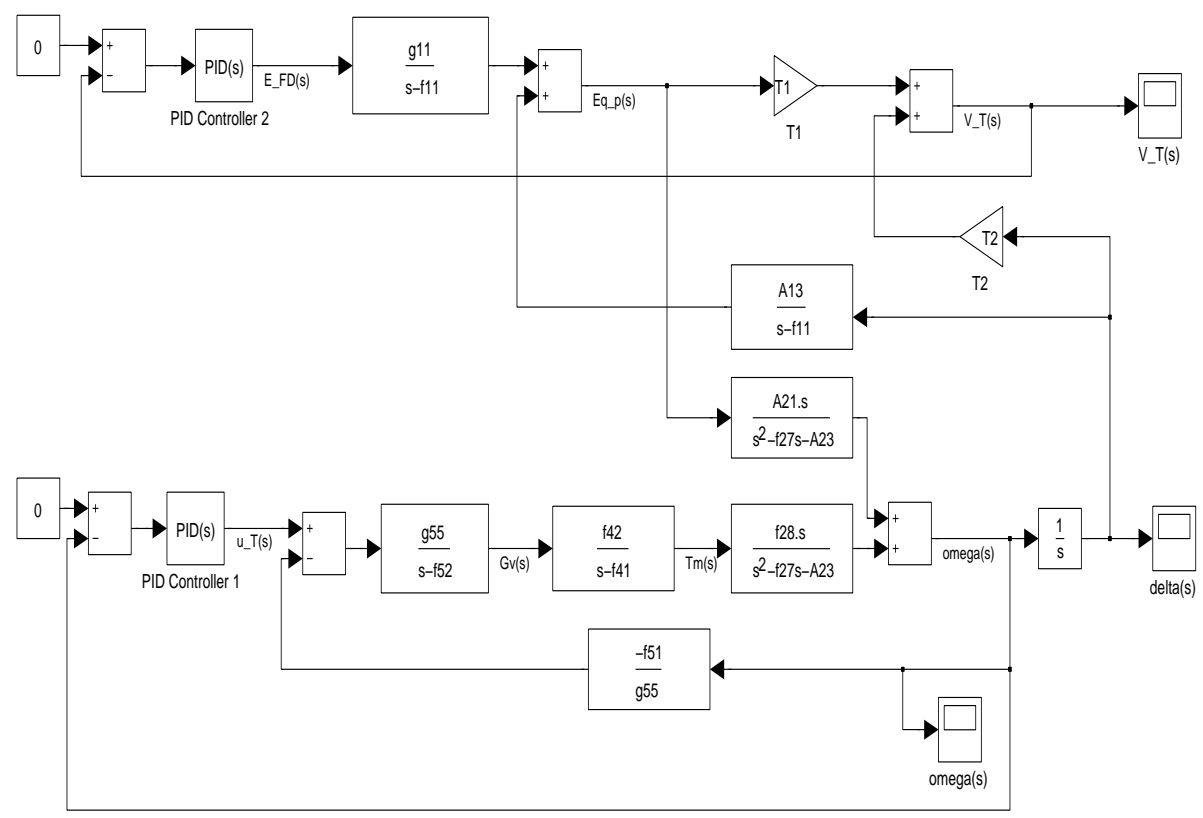

Figure 5.17: Simulink model of the PID compensated (LFC+AVR) MIMO system including coupling

\section{PID Controller Design}

\subsection{PID Controller Design based on linear model}

The two PID controllers that were designed for the LFC and the AVR SISO systems in the previous section, are now tested on the combined LFC and AVR system including coupling which is the same as the linear model. A simulink block diagram which consists of the two PID controllers and the combined LFC and AVR system including coupling is constructed in Figure 5.17. Figure 6.1 to Figure 6.5 show $\Delta \delta(\mathrm{s}), \Delta \omega(\mathrm{s})$, $\Delta V_{t}(\mathrm{~s}), \Delta E_{f d}(\mathrm{~s})$, and $\Delta u_{T}(\mathrm{~s})$ vs time plots for the PID compensated combined (LFC+AVR) MIMO system including coupling. The plots obtained when the coupling coefficients were set to zero i.e. in the decoupled LFC and AVR SISO systems, are identical to the plots obtained for the combined (LFC+AVR) MIMO system including coupling. Thus, separate frequency and voltage control of the synchronous generator and turbine-governor system connected to an infinite bus is justified. 


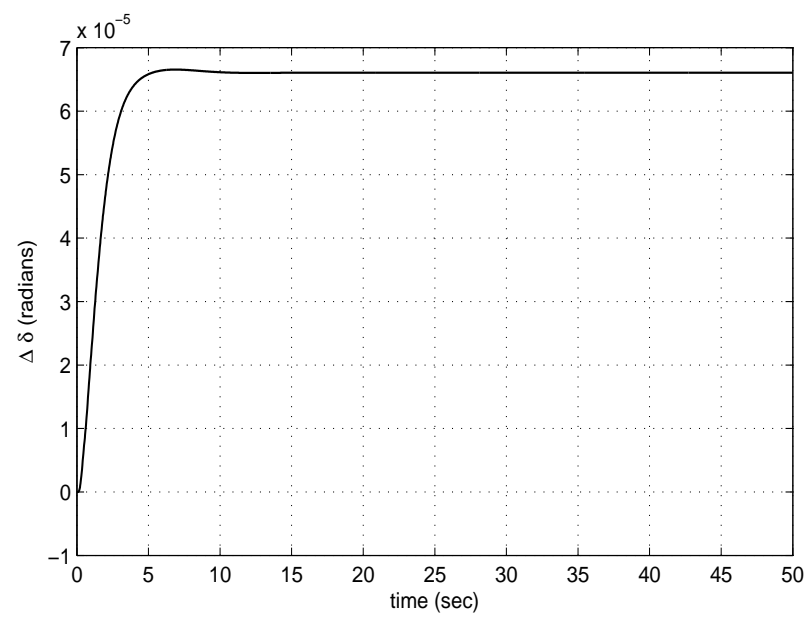

Figure 6.1: Plot of $\Delta \delta(\mathrm{s})$ vs time for the PID compensated (LFC+AVR) MIMO system including coupling

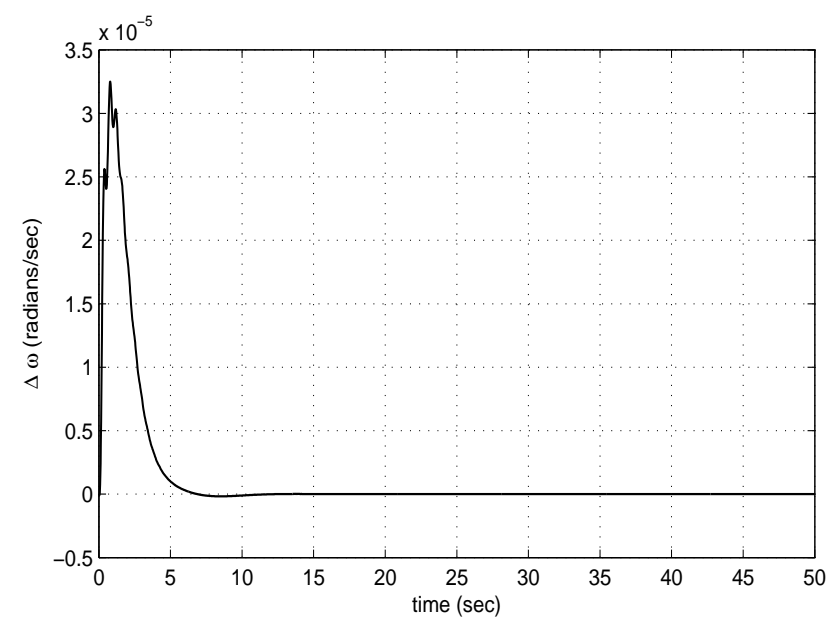

Figure 6.2: Plot of $\Delta \omega(\mathrm{s})$ vs time for the PID compensated (LFC+AVR) MIMO system including coupling

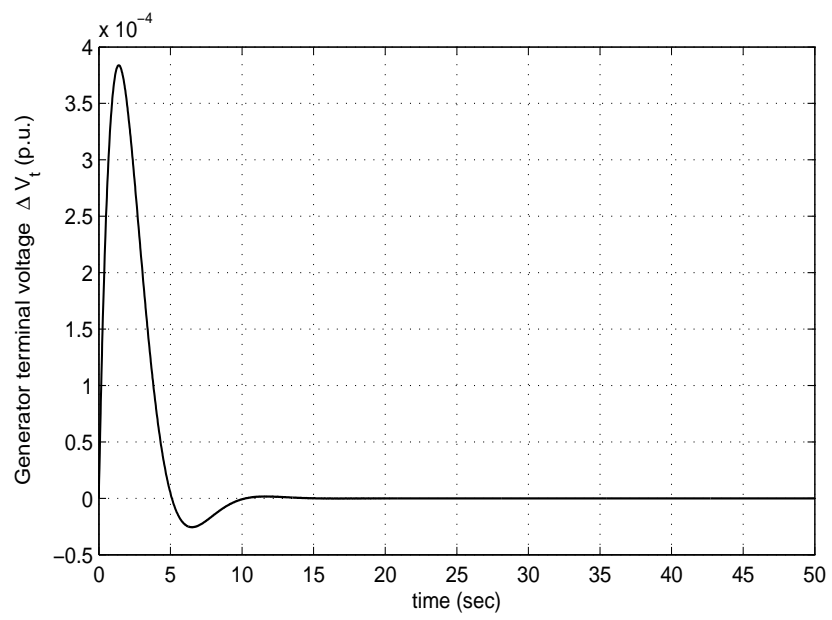

Figure 6.3: Plot of $\Delta V_{t}(\mathrm{~s})$ vs time for the PID compensated (LFC+AVR) MIMO system including coupling 


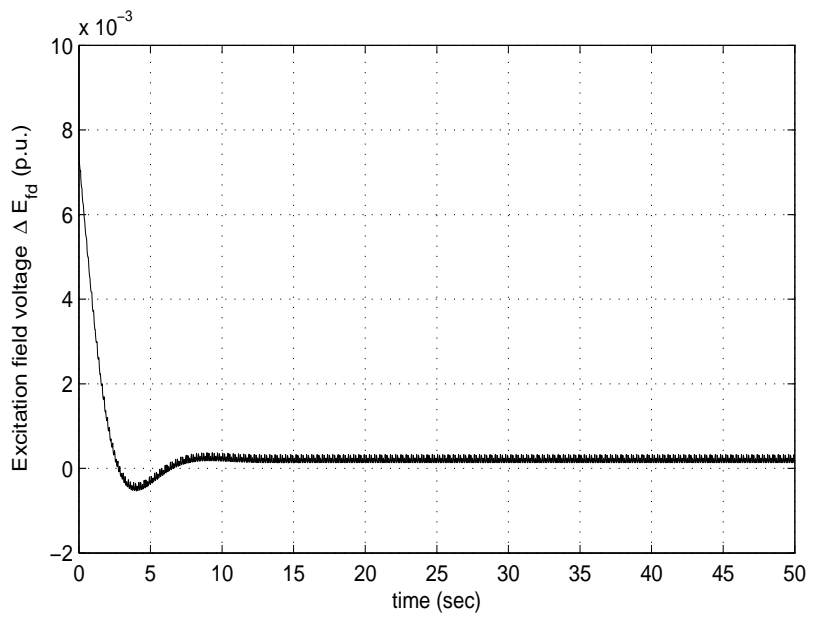

Figure 6.4: Plot of $\Delta E_{f d}(\mathrm{~s})$ vs time for the PID compensated (LFC+AVR) MIMO system including coupling

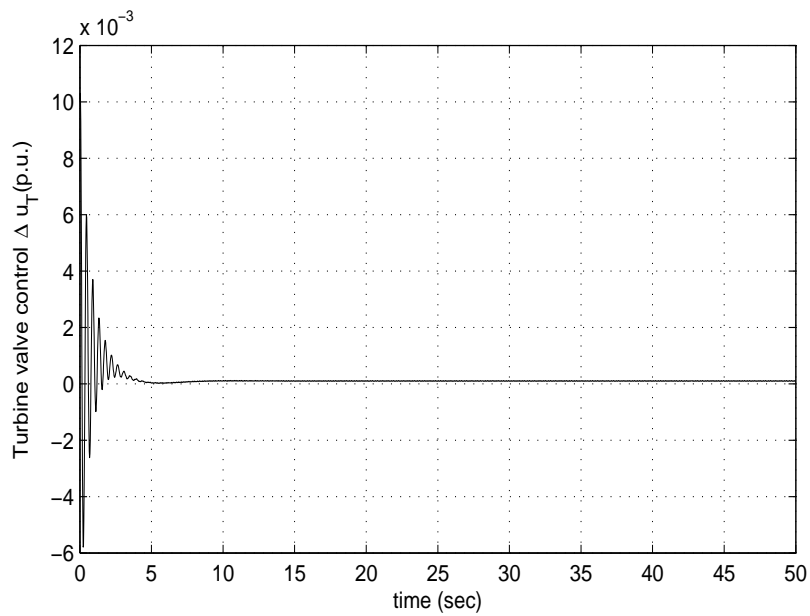

Figure 6.5: Plot of $\Delta u_{T}(\mathrm{~s})$ vs time for the PID compensated (LFC+AVR) MIMO system including coupling 


\subsection{Simulation results for the PID Controllers applied to the Reduced Order Nonlinear Model}

The two PID controllers that were designed for the decoupled LFC and AVR SISO systems and then tested on the (LFC+AVR) MIMO system including coupling are now tested on the reduced order nonlinear model. Figure 6.6 to Figure 6.11 show simulation results for the two PID controllers that are tested on the reduced order nonlinear model. From these results we can see that all the state variables, outputs, and the control inputs attain their respective steady state values in approximately 10 seconds. 


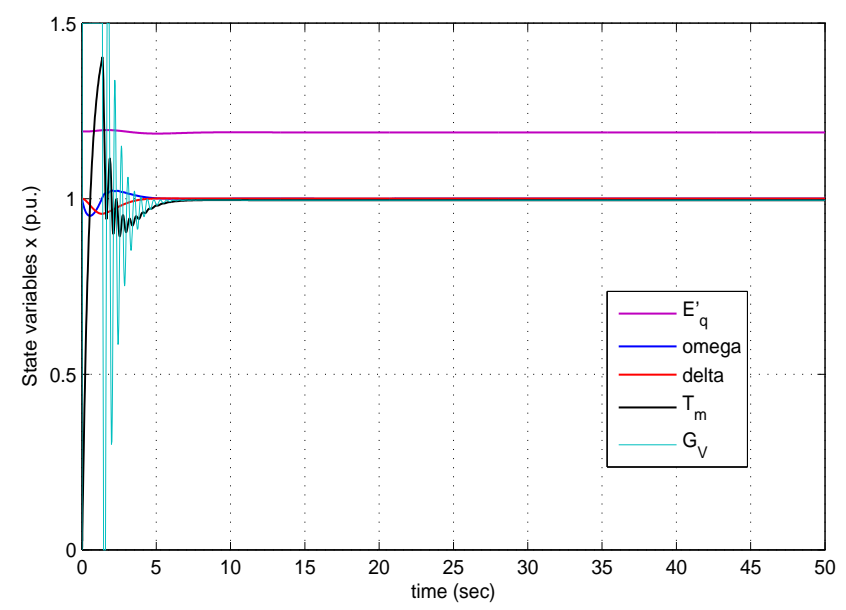

Figure 6.6: Plot of the state variables $E_{q}^{\prime}, \omega, \delta, T_{m}$, and $G_{V}$ vs time for the PID controller applied to the reduced order nonlinear model

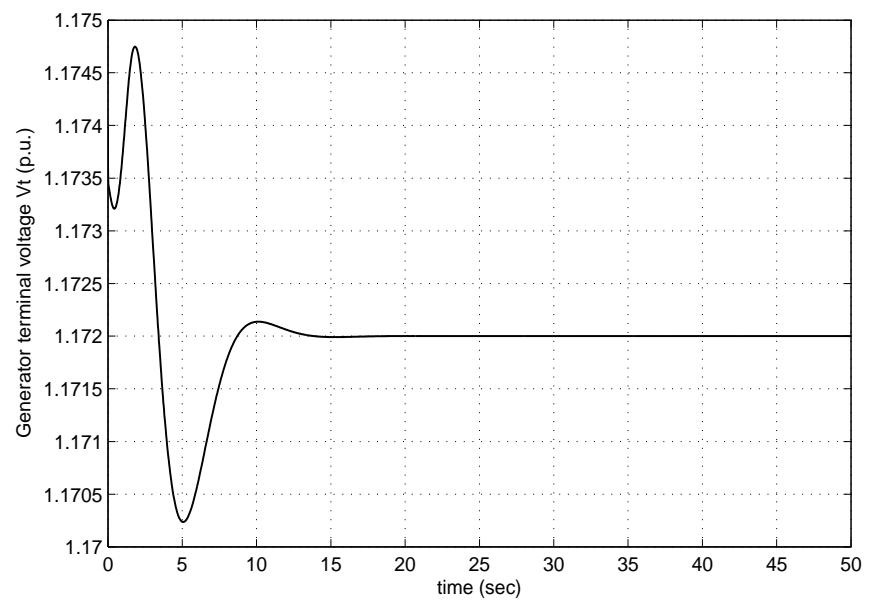

Figure 6.7: Plot of the generator terminal voltage $V_{t}$ vs time for the PID controller applied to the reduced order nonlinear model

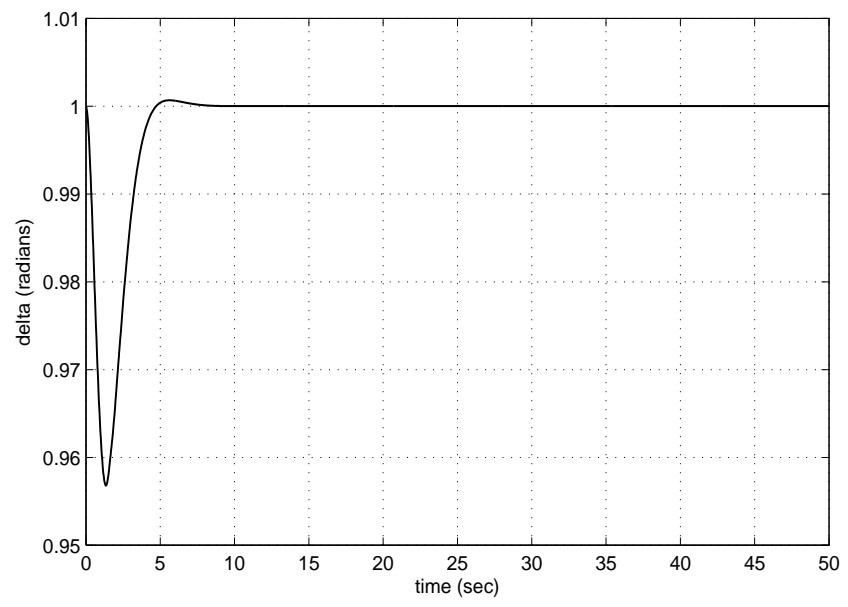

Figure 6.8: Plot of the rotor angle $\delta$ vs time for the PID controller applied to the reduced order nonlinear model 


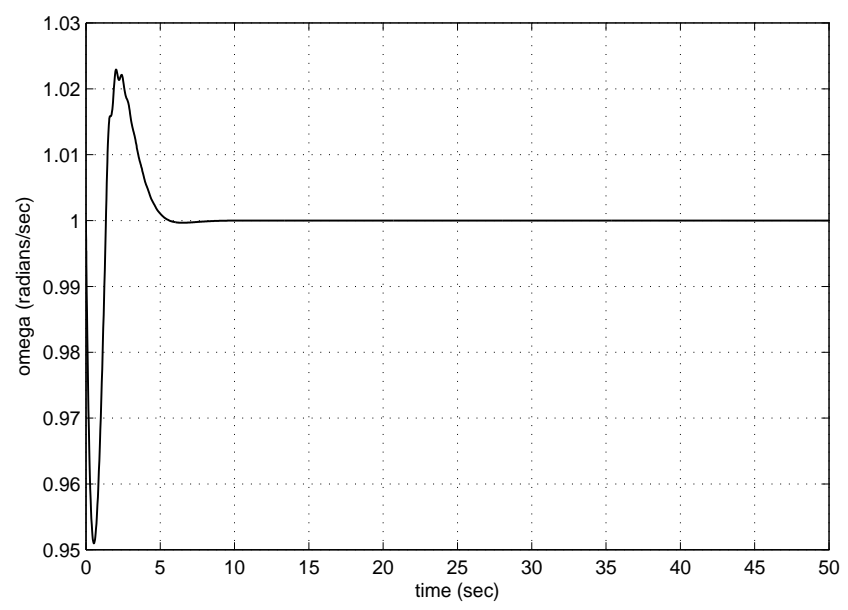

Figure 6.9: Plot of the frequency $\omega$ vs time for the PID controller applied to the reduced order nonlinear model

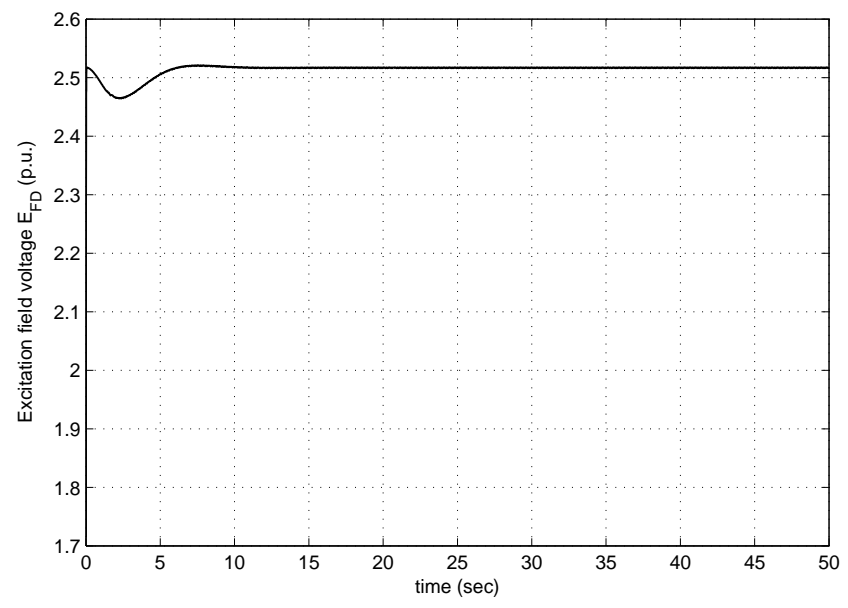

Figure 6.10: Plot of the generator excitation field control $E_{F D}$ vs time for the PID controller applied to the reduced order nonlinear model

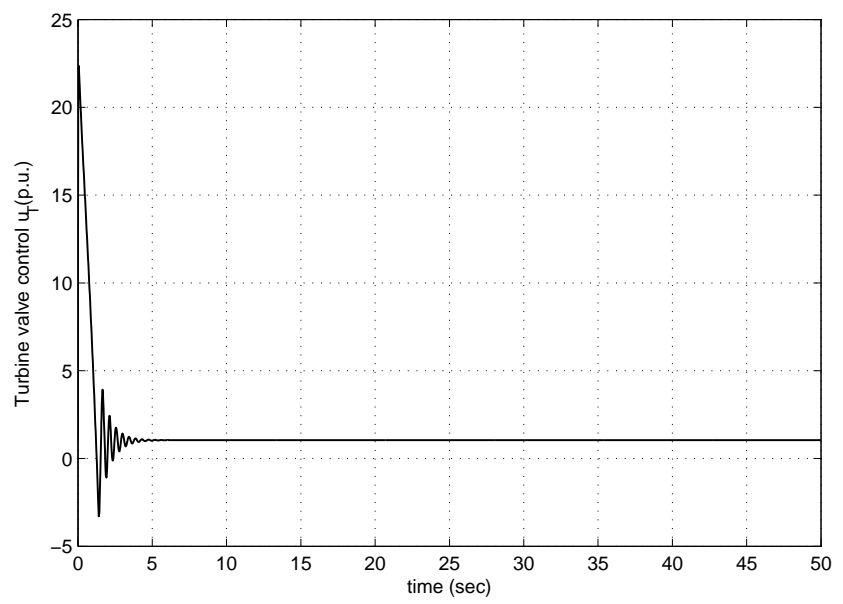

Figure 6.11: Plot of the turbine valve control $u_{T}$ vs time for the PID controller applied to the reduced order nonlinear model 


\subsection{Simulation results for the PID Controllers applied to the Truth Model}

The two PID controllers that were designed for the decoupled reduced order LFC and AVR SISO systems, and then tested on the (LFC+AVR) MIMO system including coupling and the reduced order nonlinear model, are now tested on the truth model. The PID controller which controls the LFC loop is re-tuned to $K_{p 1}=2000, K_{i 1}=1500$, and $K_{d 1}=1000$. Whereas, the PID controller which controls the AVR loop is re-tuned to $K_{p 2}=2000, K_{i 2}=15000$, and $K_{d 2}=4$. Figure 6.12 to Figure 6.16 show simulation results for the two PID controllers that are tested on the truth model. From these simulation results we can see that the generator terminal voltage $V_{t}$, angular velocity $\omega$, rotor angle $\delta$, and the two control inputs $V_{F}$ and $u_{T}$, settle to their respective steady state values. 


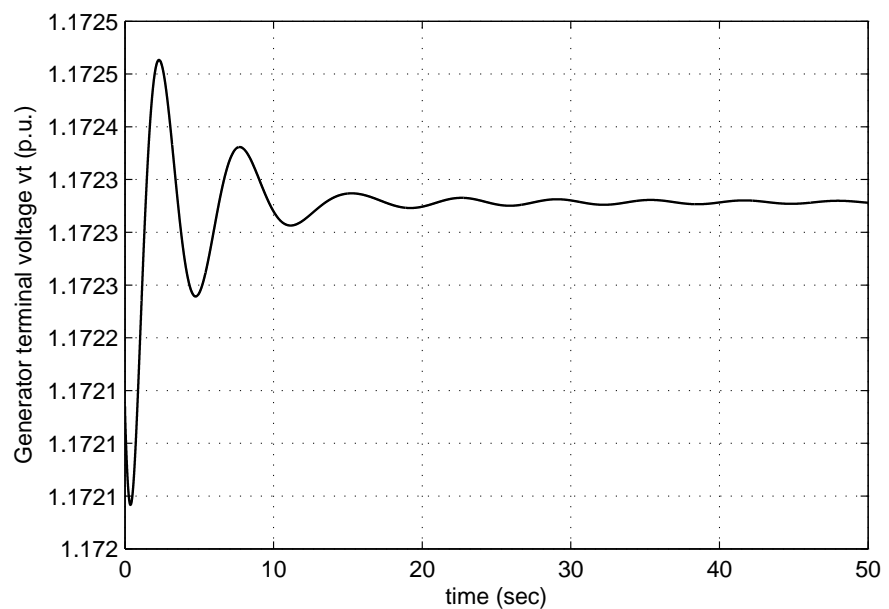

Figure 6.12: Plot of the generator terminal voltage $V_{t}$ vs time for the PID controllers applied to the truth model

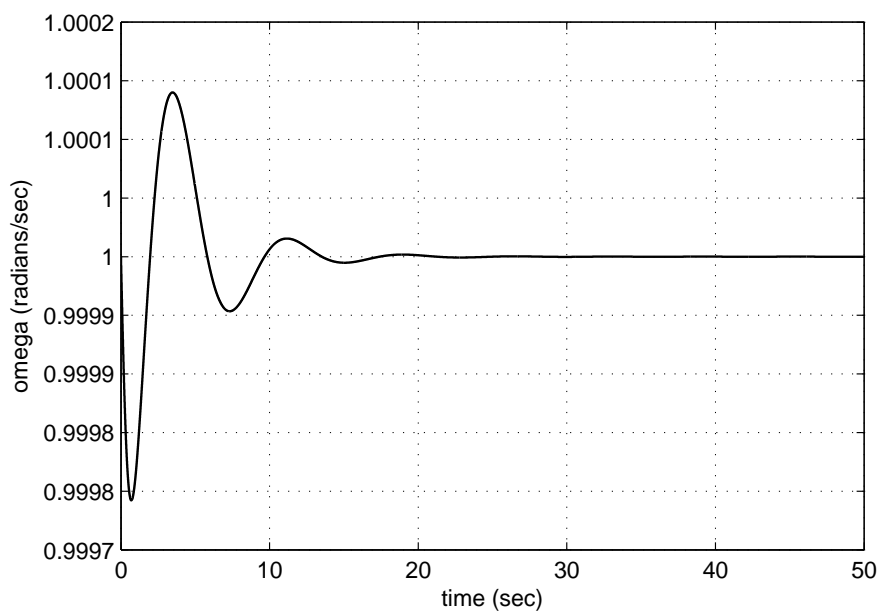

Figure 6.13: Plot of the angular velocity $\omega$ vs time for the PID controllers applied to the truth model

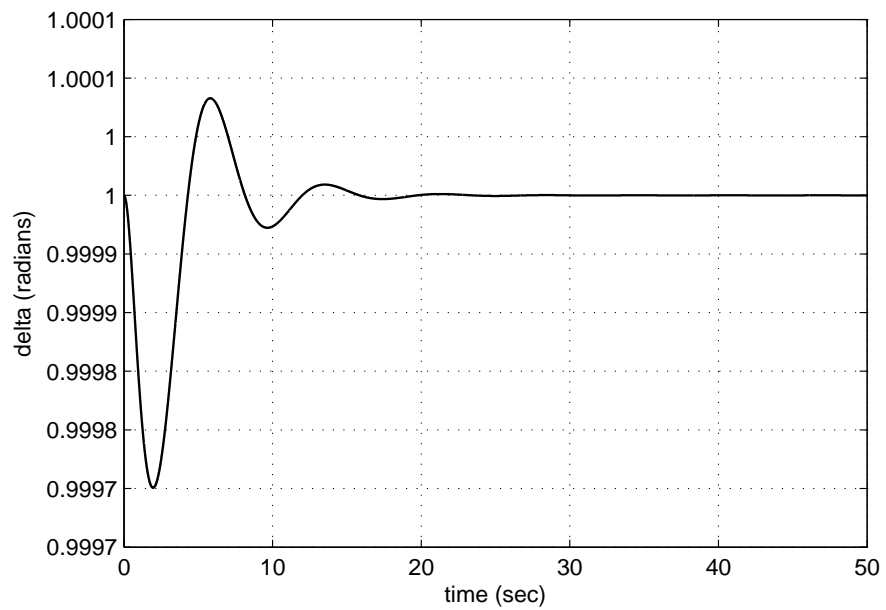

Figure 6.14: Plot of the rotor angle $\delta$ vs time for the PID controllers applied to the truth model 


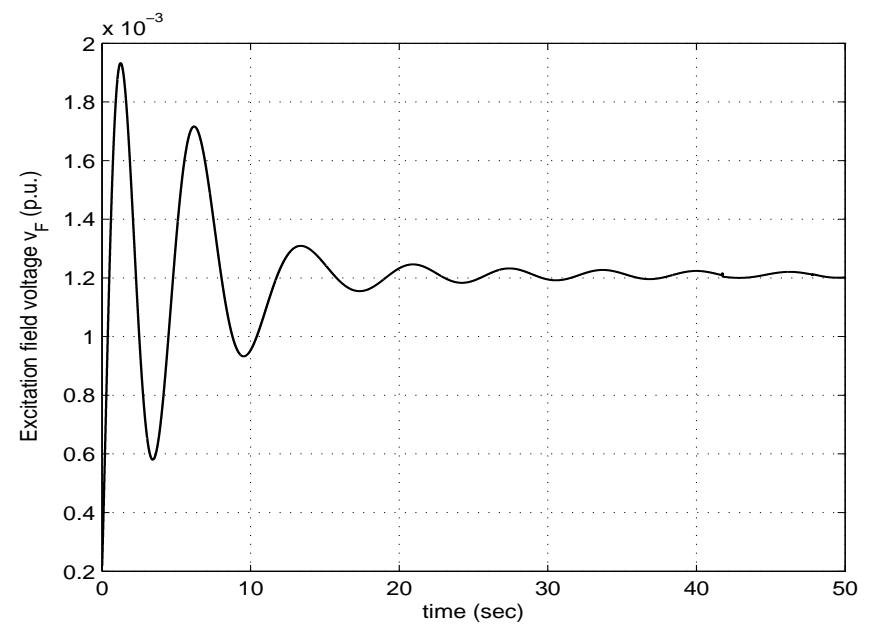

Figure 6.15: Plot of the control input $V_{F}$ vs time for the PID controllers applied to the truth model

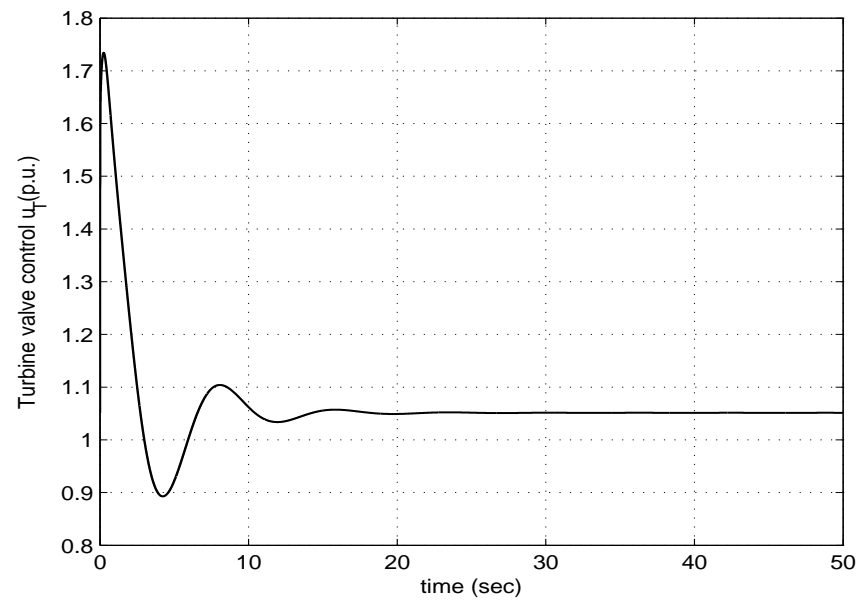

Figure 6.16: Plot of the control input $u_{T}$ vs time for the PID controllers applied to the truth model 


\section{Linear State-Space Controller Design}

In this section we design linear state-space controllers for the synchronous generator and turbine connected to an infinite bus.

\subsection{State Feedback Controller design using LQR methodology}

\subsubsection{LQR Design based on linear model}

We first design a linear-quadratic regulator (LQR) for the linearized model of the synchronous generator and turbine connected to an infinite bus. Let us assume that we have sensors to measure all the states and we use a full-state feedback controller (regulator) of the form

$$
\mathbf{u}=-K \mathbf{x}
$$

that seeks to drive the states to zero. Since, the system is MIMO with 2 inputs and 5 states, LQR controller design entails finding the $2 \times 5$ gain vector $K$. This can be done by directly using the lqr command in MATLAB. For this, letting

$$
J=\int_{0}^{\infty}\left(\mathbf{x}^{T} Q \mathbf{x}+\mathbf{u}^{T} R \mathbf{u}\right) d t
$$

we seek to find the gain vector $K$ to minimize the cost function $J$. Minimization of $J$ results in driving $\mathbf{x}(t)$ to zero with as little control energy and state deviations as possible, with the balance between control energy and state deviations specified via the $Q$ and $R$ matrices. Here, assume the $5 \times 5 Q$ matrix is diagonal with diagonal elements $q_{i} \geq 0$ (each providing a weight for a different element of the deviation of the state) and the $2 \times 2 R$ matrix is diagonal with diagonal elements $r_{i}>0$ (each providing a weight for the deviation of the two control inputs. The values for $Q$ and $R$ are used as design parameters.

A methodology to tune the $Q$ and $R$ matrices is given as follows: If all the $q_{i}=0$, then the excursions of the states are high while the control input tries to force the state to zero. High values of $q_{i}$ relative to $r_{i}$ mean that you are willing to use lots of control energy to keep state excursions small while driving it to zero. Clearly, you cannot pick $r_{i}=0$ as this results in allowing infinite control energy to force the state to zero, typically then very fast. Finding the gain $K$ to minimize $J$ involves solving the Riccati equation $A^{T} P+P A-P B R^{-1} B^{T} P+Q=0$, where $K=R^{-1} B^{T} P$. We use the Matlab lqr command to directly solve for the gain vector $K$ given $A, B, Q$, and $R$. Thus, by using the weighting matrices

$$
Q=\left[\begin{array}{ccccc}
300 & 0 & 0 & 0 & 0 \\
0 & 250 & 0 & 0 & 0 \\
0 & 0 & 200 & 0 & 0 \\
0 & 0 & 0 & 200 & 0 \\
0 & 0 & 0 & 0 & 250
\end{array}\right]
$$

and

$$
R=\left[\begin{array}{cc}
0.5 & 0 \\
0 & 0.5
\end{array}\right]
$$

and the state space matrices $(A, B)$ as given in Equation (3.76) and Equation (3.77) the control gain $K$ is found to be

$$
K=\left[\begin{array}{ccccc}
23.7240 & -36.3457 & -5.5938 & -2.5612 & -0.0454 \\
-1.3381 & 21.0340 & 1.5703 & 9.0242 & 21.5437
\end{array}\right]
$$

Figure 7.1 shows time history of the state variables $\left(\Delta E_{q}^{\prime}, \Delta \omega, \Delta \delta, \Delta T_{m}\right.$, and $\left.\Delta G_{V}\right)$ for the full-state feedback LQR controller applied to the reduced order linear model. From this plot we can see that the LQR drives all the states to 0 in approximately 20 to 25 seconds. Similarly, from Figure 7.2 to Figure 7.6 we can see that $\Delta V_{t}, \Delta \omega, \Delta \delta, \Delta E_{f d}$, and $\Delta u_{T}$ converge to zero in approximately 25 seconds. 


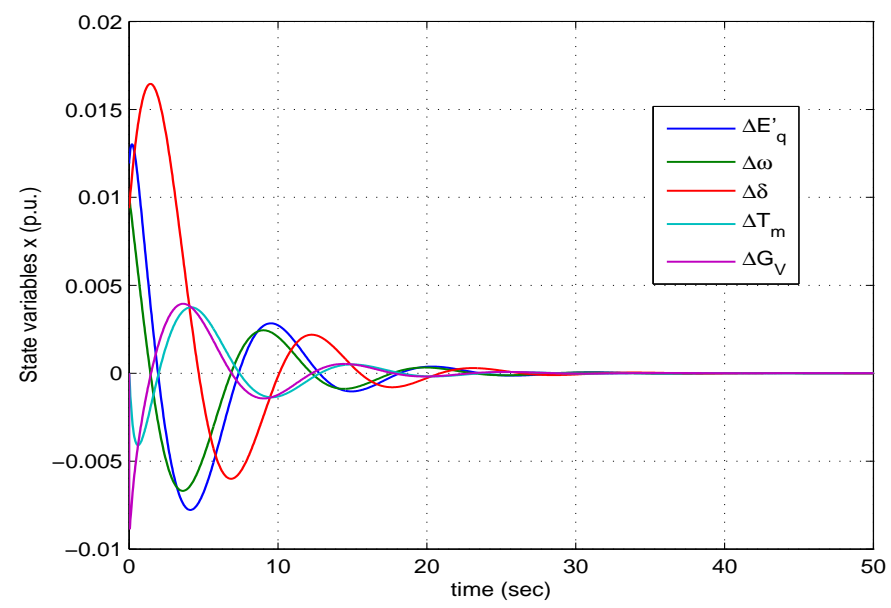

Figure 7.1: Plot of the state variables $\Delta E_{q}^{\prime}, \Delta \omega, \Delta \delta, \Delta T_{m}$, and $\Delta G_{V}$ vs time for the full-state feedback LQR applied to the reduced order linear model

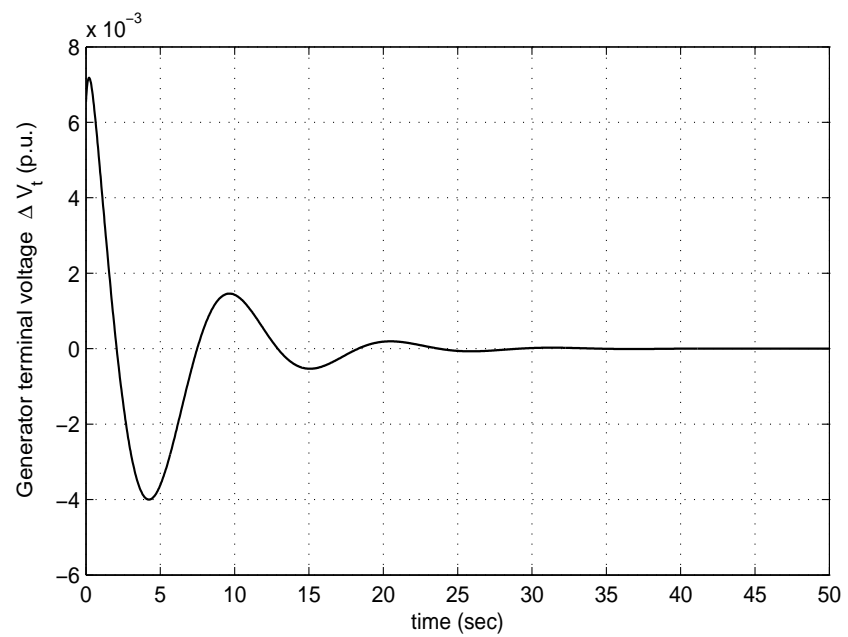

Figure 7.2: Plot of the generator terminal voltage $\Delta V_{t}$ vs time for the full-state feedback LQR applied to the reduced order linear model

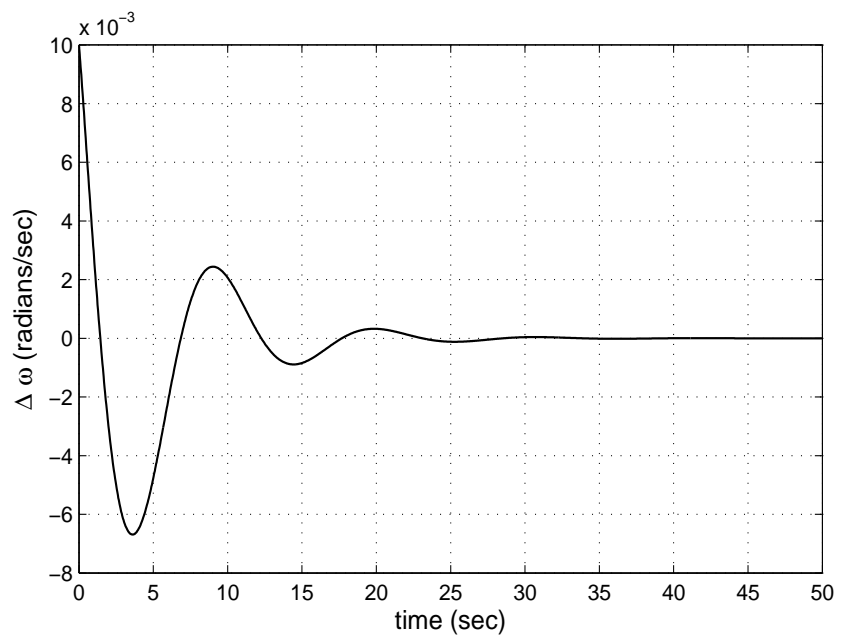

Figure 7.3: Plot of $\Delta \omega$ vs time for the full-state feedback LQR applied to the reduced order linear model 


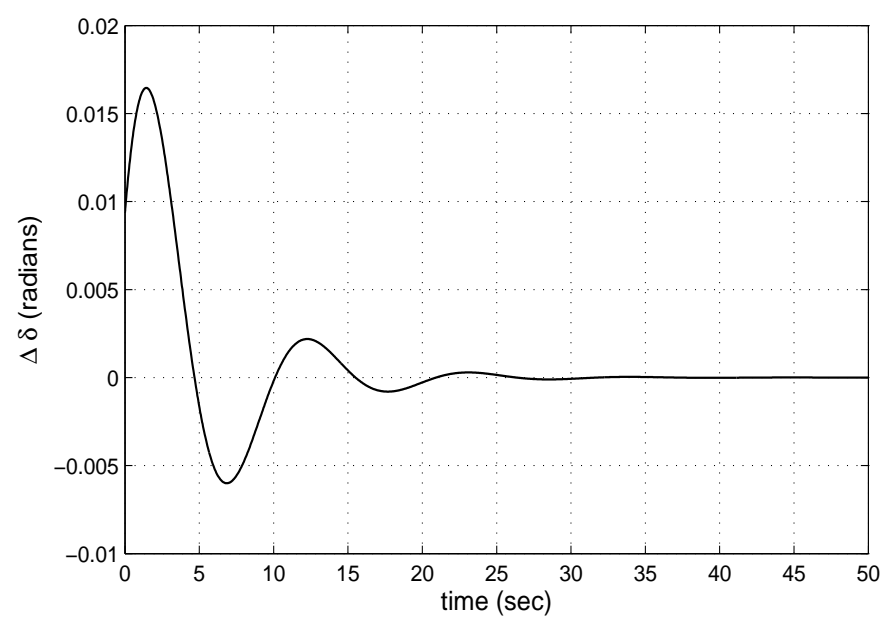

Figure 7.4: Plot of $\Delta \delta$ vs time for the full-state feedback LQR applied to the reduced order linear model

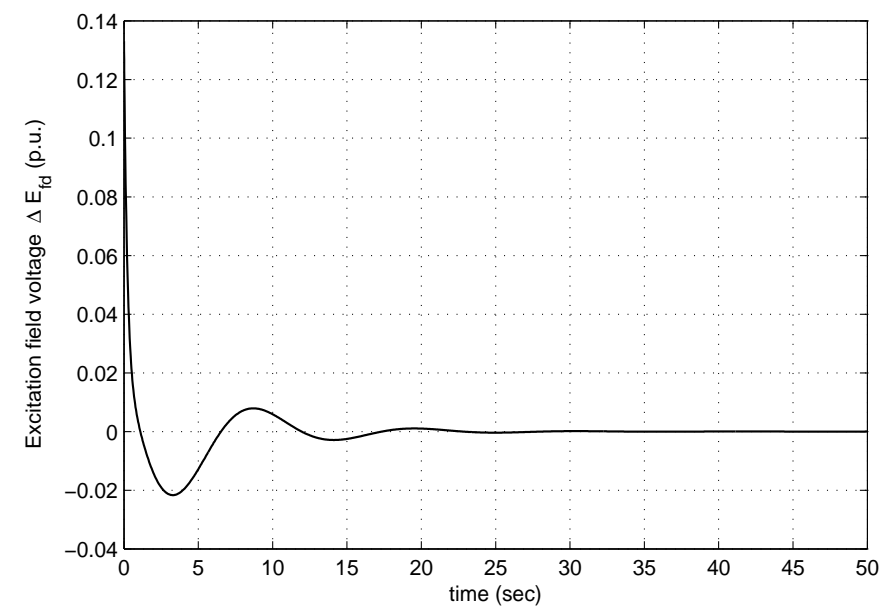

Figure 7.5: Plot of the control input $\Delta E_{f d}$ vs time for the full-state feedback LQR applied to the reduced order linear model

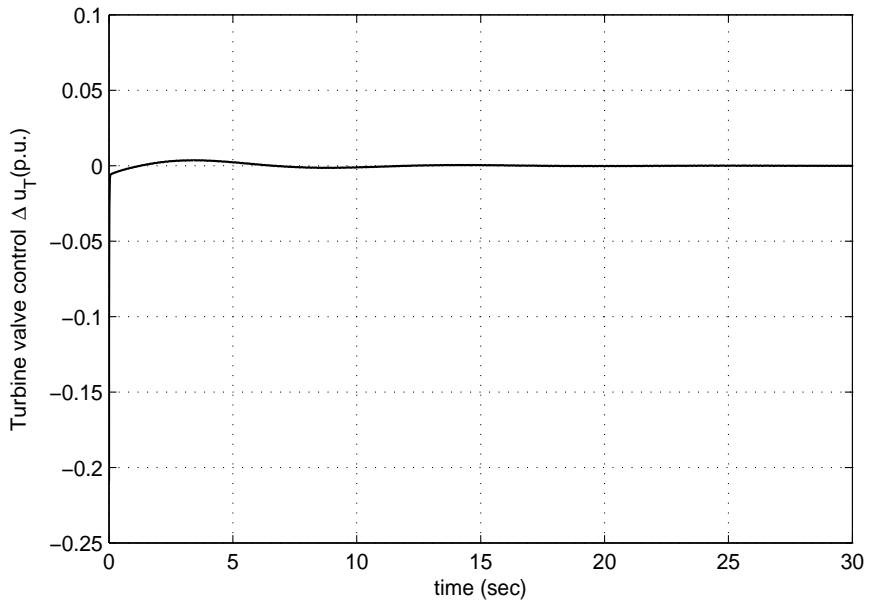

Figure 7.6: Plot of the control input $\Delta u_{T}$ vs time for the full-state feedback LQR applied to the reduced order linear model 


\subsubsection{Simulation results for the Full-State Feedback LQR applied to the Reduced Order Nonlinear Model}

The LQR based full-state feedback controller that was earlier designed for the reduced order linear model is now tested on the reduced order nonlinear model. Figure 7.7 and Figure 7.8 show plots for the generator terminal voltage $V_{t}$, and rotor angle $\delta$, respectively. The generator terminal voltage $V_{t}$ settles to a new steady state value of 1.13 p.u. which deviates from the desired steady state value of 1.1723 p.u. by an amount of 0.0423 p.u. Similarly, the rotor angle $\delta$ settles to a new steady state value of 1.056 p.u. which deviates from the desired steady state value of 1 p.u. by an amount of 0.056 p.u. We observe a steady state error when the LQR with original gains of subsection 7.1.1 is applied to the reduced order nonlinear model. Therefore, the gains of the controller are tuned once again so that the controller works efficiently on the reduced order nonlinear model. We use the Matlab lqr command to directly solve for the gain vector $K$ given $A, B, Q$, and $R$. The system matrices $(A, B)$ are evaluated at the nominal operating point, and $Q$ and $R$ matrices are appropriately tuned as per the procedure explained in the LQR design section. Thus, by using the feedback law,

$$
\mathbf{u}=-K \mathbf{x}
$$

the weighting matrices

$$
Q=\left[\begin{array}{ccccc}
40000 & 0 & 0 & 0 & 0 \\
0 & 10000 & 0 & 0 & 0 \\
0 & 0 & 250000 & 0 & 0 \\
0 & 0 & 0 & 500 & 0 \\
0 & 0 & 0 & 0 & 500
\end{array}\right]
$$

and

$$
R=\left[\begin{array}{cc}
0.07 & 0 \\
0 & 0.07
\end{array}\right]
$$

and the state space matrices $(A, B)$ as given in Equation (3.76) and Equation (3.77) the control gain $K$ is found to be

$$
K=\left[\begin{array}{ccccc}
753.9172 & -575.5829 & -610.0649 & -27.6436 & -0.1301 \\
-3.8375 & 1782.567 & 1474.307 & 128.4938 & 84.1272
\end{array}\right]
$$

Figure 7.9 to Figure 7.14 show simulation results for the LQR-based full-state feedback controller applied to the reduced order nonlinear model with the gains re-tuned. From these results we can see that all the state variables, and outputs, attain their respective steady state values in approximately 8-10 seconds. Figure 7.13 and Figure 7.14 show plots of the two control inputs, $E_{f d}$ and $u_{T}$, respectively. 


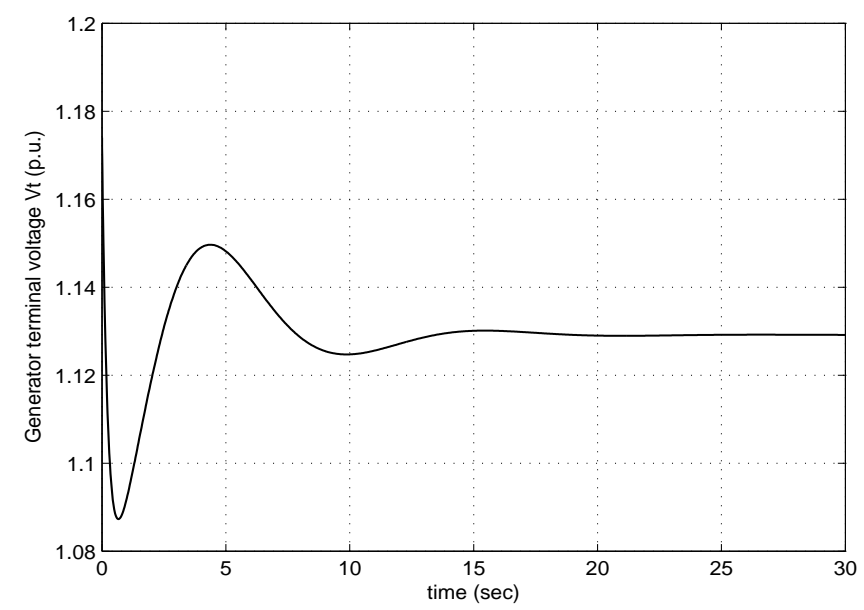

Figure 7.7: Plot of the generator terminal voltage $V_{t}$ vs time for the LQR-based full-state feedback controller with the original gains of subsection 7.1.1 applied to the reduced order nonlinear model

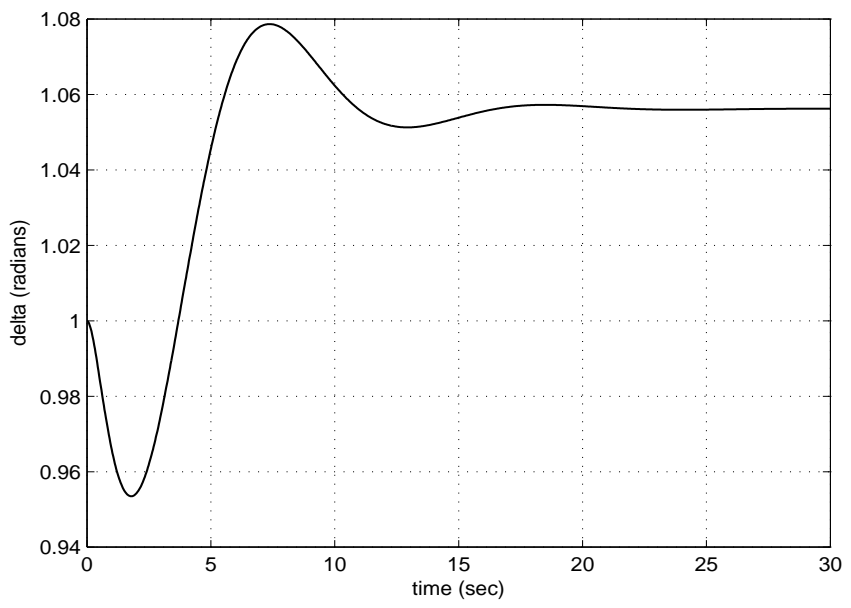

Figure 7.8: Plot of the rotor angle $\delta$ vs time for the LQR-based full-state feedback controller with the original gains of subsection 7.1.1 applied to the reduced order nonlinear model

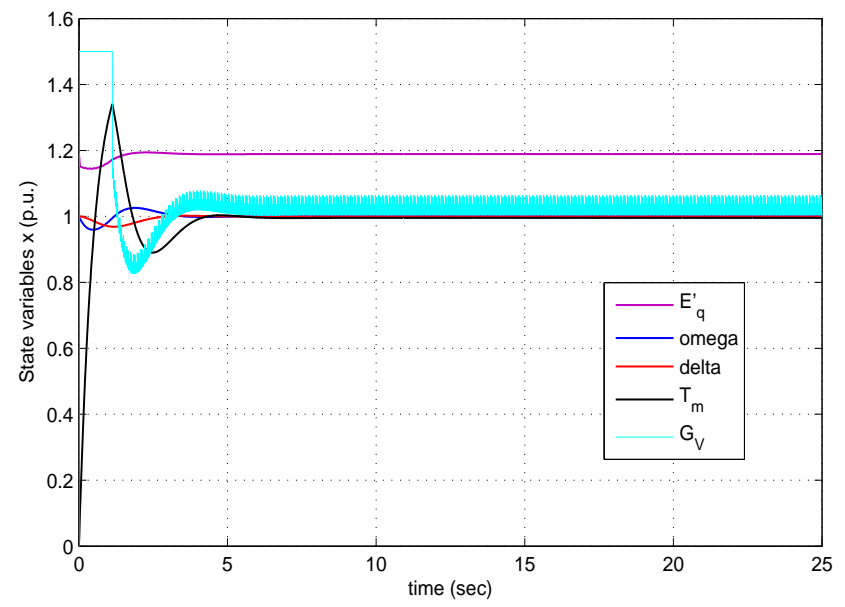

Figure 7.9: Plot of the state variables $E_{q}^{\prime}, \omega, \delta, T_{m}$, and $G_{V}$ vs time for the re-tuned LQR-based full-state feedback controller applied to the reduced order nonlinear model 


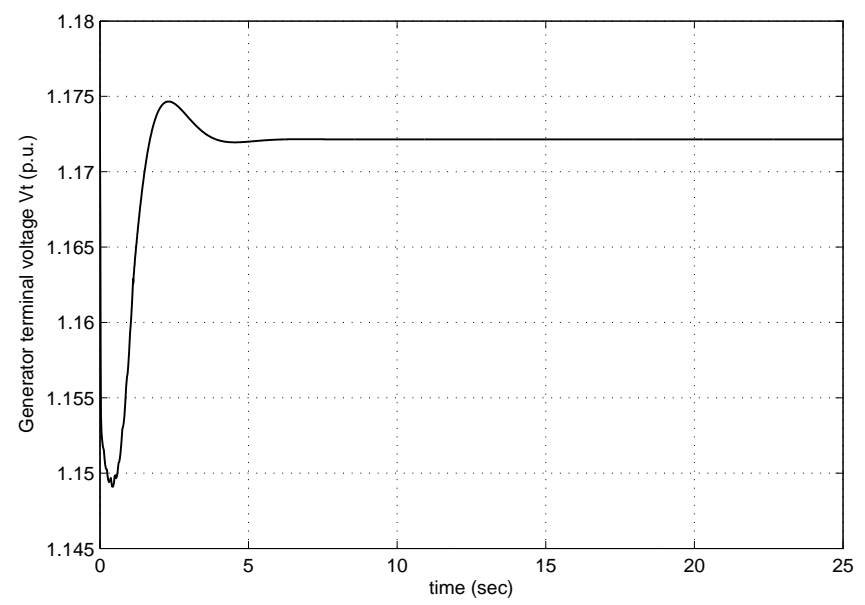

Figure 7.10: Plot of the generator terminal voltage $V_{t}$ vs time for the re-tuned LQR-based full-state feedback controller applied to the reduced order nonlinear model

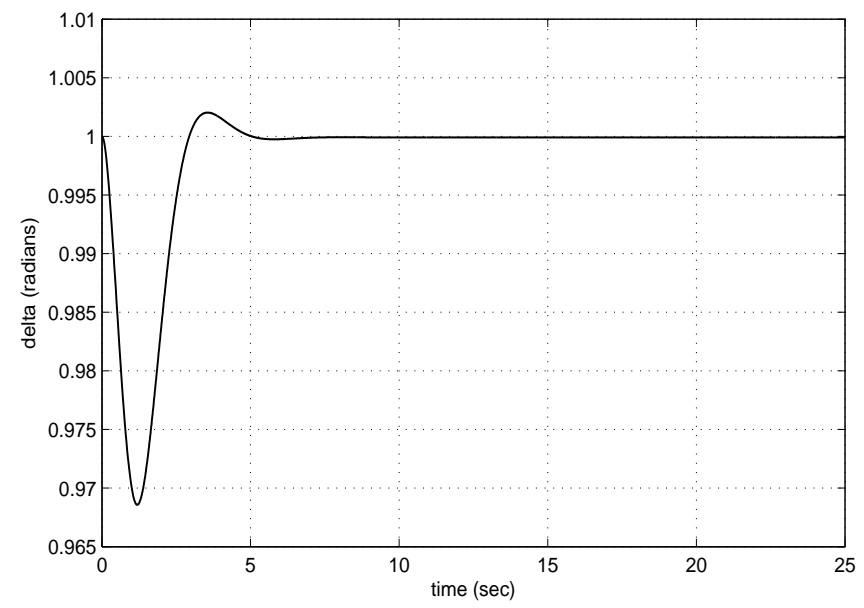

Figure 7.11: Plot of the rotor angle $\delta$ vs time for the re-tuned LQR-based full-state feedback controller applied to the reduced order nonlinear model

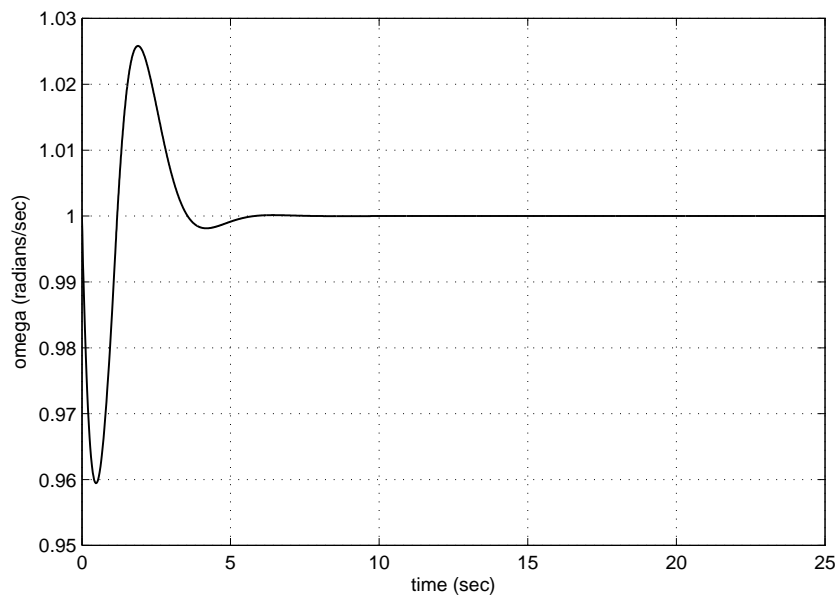

Figure 7.12: Plot of the frequency $\omega$ vs time for the re-tuned LQR-based full-state feedback controller applied to the reduced order nonlinear model 


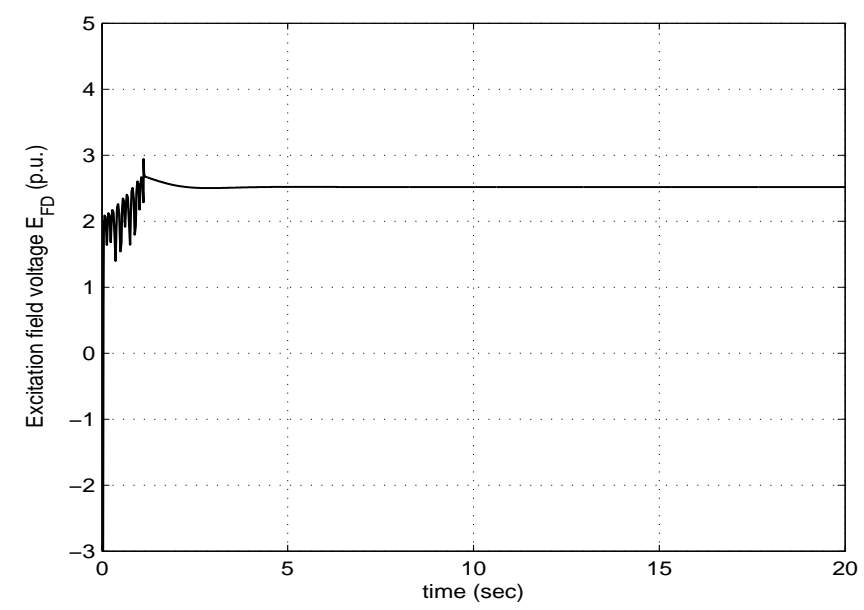

Figure 7.13: Plot of the control input $E_{f d}$ vs time for the full-state feedback LQR applied to the reduced order nonlinear model

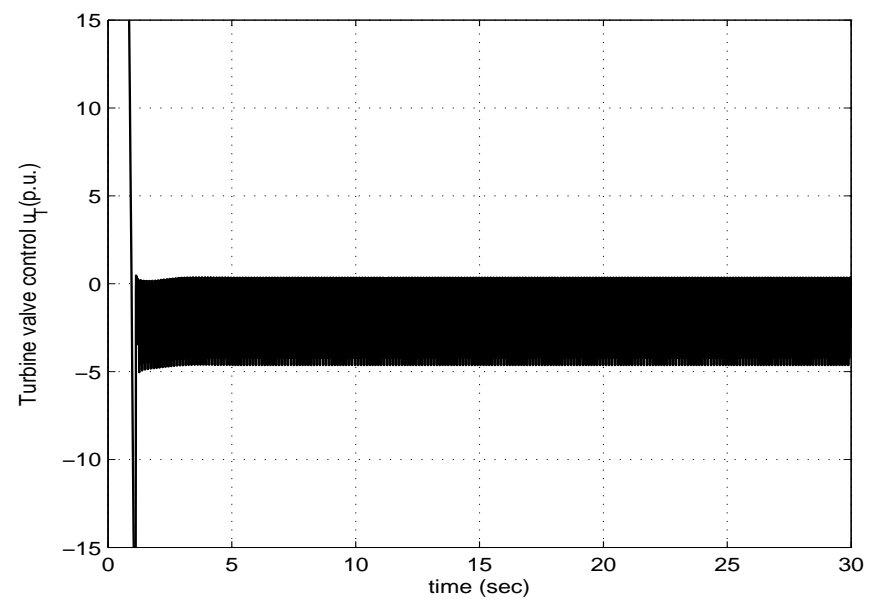

Figure 7.14: Plot of the control input $u_{T}$ vs time for the full-state feedback LQR applied to the reduced order nonlinear model 


\subsubsection{Simulation results for the Full-State Feedback LQR applied to the Truth Model}

The LQR-based full-state feedback controller that was designed for the reduced order linear model and then re-tuned and tested on the reduced order nonlinear model, is now tested on the truth model. There is a non physical state $E_{q}^{\prime}$ in the reduced order model that needs to be reconstructed from the states of the truth model. The LQR-based full-state feedback controller can be implemented on the truth model by using either Equation (7.10) or Equation (7.11) to express $E_{q}^{\prime}$ as a function of state variables of the truth model. The gains of this controller are the same as the LQR controller that was re-tuned and tested on the reduced order nonlinear model. Figure 7.15 to Figure 7.19 show simulation results for the LQR based full state feedback controller applied to the truth model. From these simulation results we can see that the generator terminal voltage $V_{t}$ oscillates about a steady state value of $1.1705 \mathrm{p} . \mathrm{u}$. which deviates from the desired steady state value of 1.1723 p.u. by an amount of 0.0018 p.u. Angular velocity $\omega$, oscillates about its desired steady state value of 1 p.u., and the rotor angle $\delta$, oscillates about its desired state value of 1 p.u. These oscillations decay with time. Also, the generator excitation voltage $V_{F}$ settles to its steady state value of 0.00121 p.u. and the turbine valve control settles to its steady state value of $1.0512 \mathrm{p} . \mathrm{u}$.

$$
\begin{gathered}
E_{q}^{\prime}=\frac{e_{14}}{e_{11}} I_{F}+\frac{e_{12}}{e_{11}} \cos (\delta-\alpha)+\frac{e_{13}}{e_{11}} \sin (\delta-\alpha) \\
E_{q}^{\prime}=e_{14} I_{F}+L_{2} I_{d}
\end{gathered}
$$




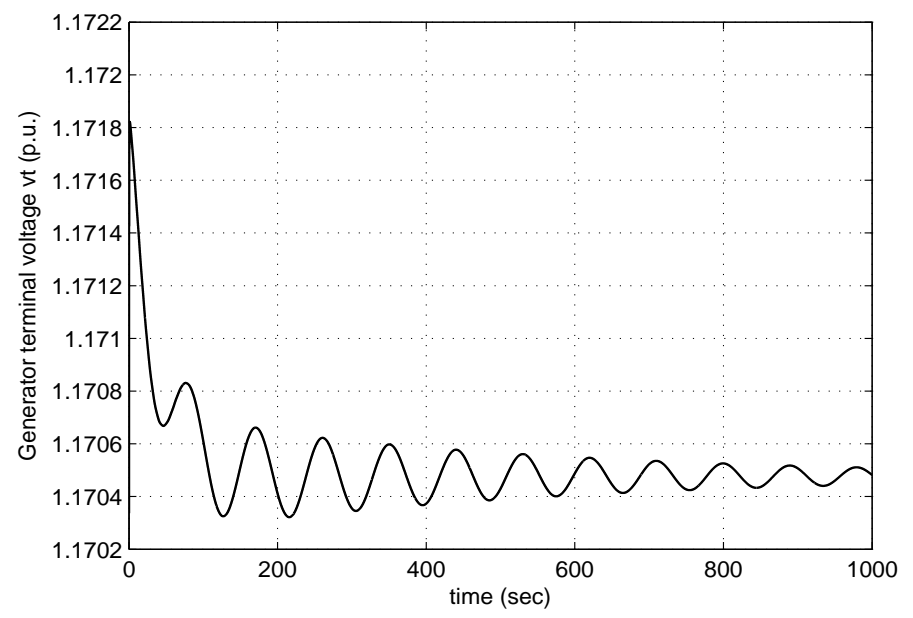

Figure 7.15: Plot of the generator terminal voltage $V_{t}$ vs time for the LQR-based full-state feedback controller applied to the truth model

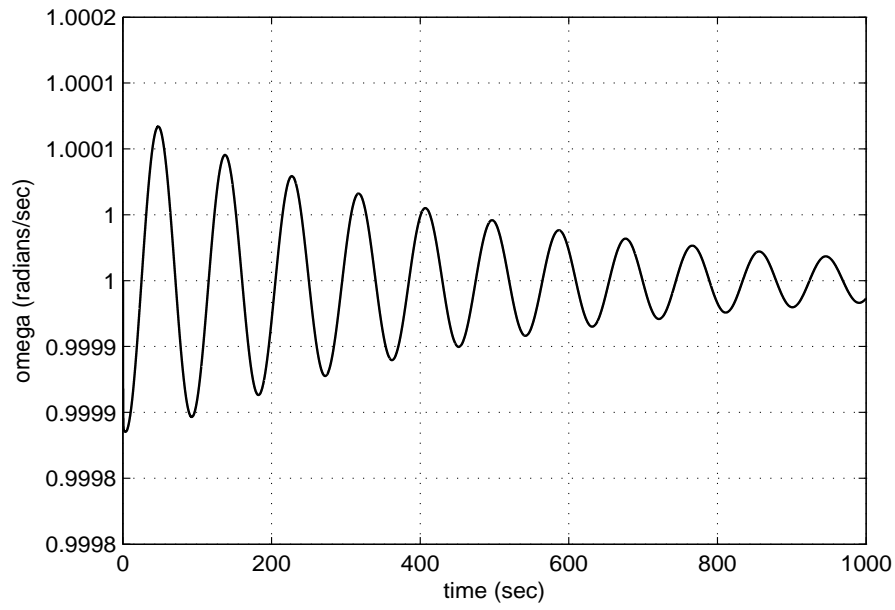

Figure 7.16: Plot of the angular velocity $\omega$ vs time for the LQR-based full-state feedback controller applied to the truth model

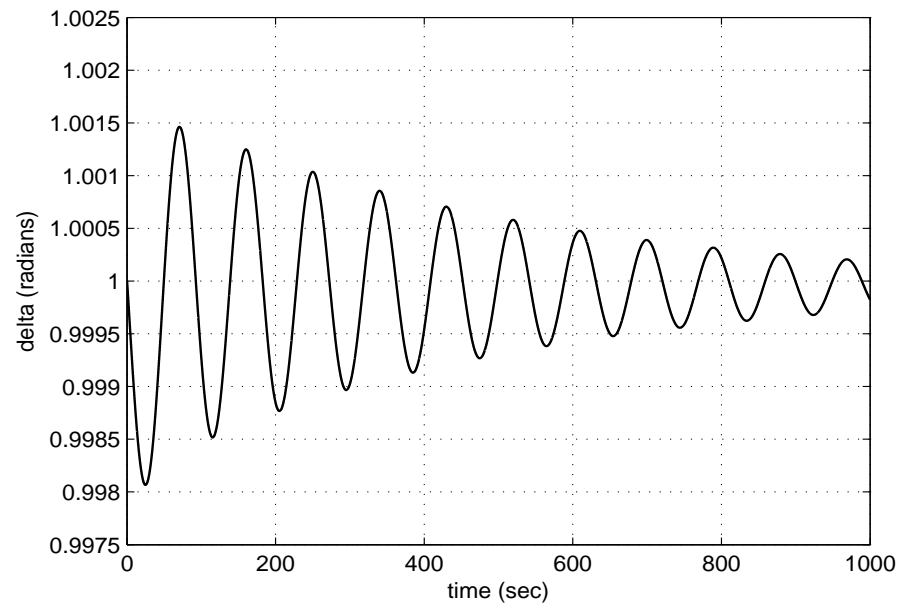

Figure 7.17: Plot of the rotor angle $\delta$ vs time for the LQR-based full-state feedback controller applied to the truth model 


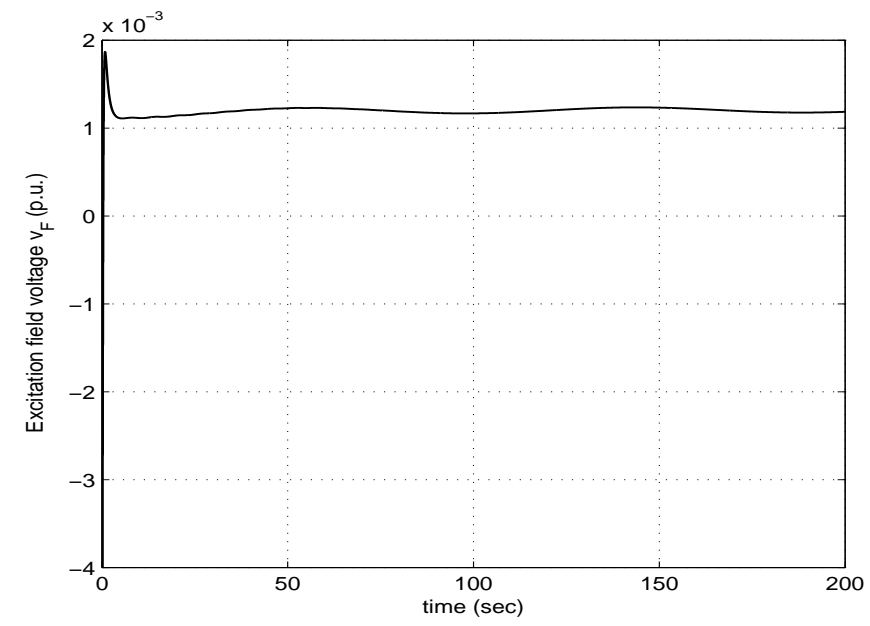

Figure 7.18: Plot of the control input $V_{F}$ vs time for the full-state feedback LQR applied to the truth model

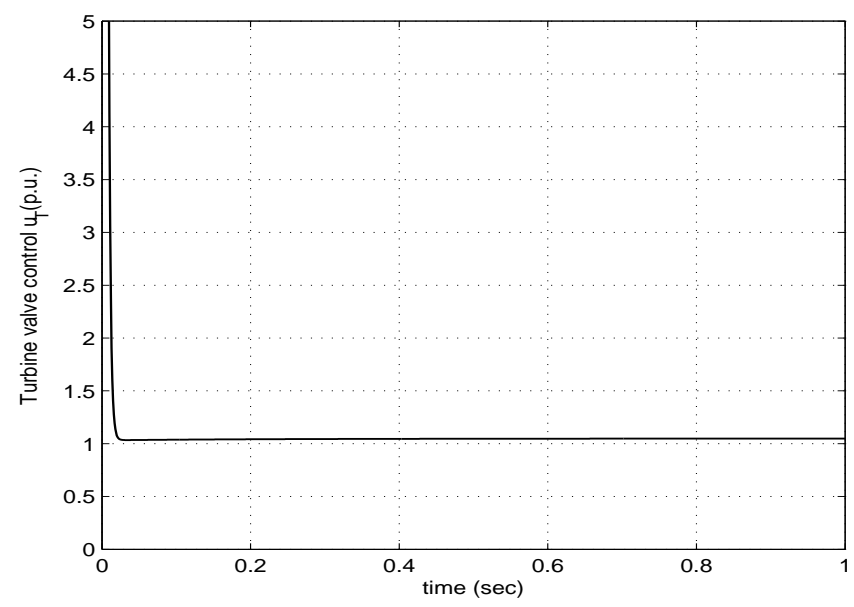

Figure 7.19: Plot of the control input $u_{T}$ vs time for the full-state feedback LQR applied to the truth model 


\subsection{State Feedback Controller design using Pole Placement Technique}

\subsubsection{Pole Placement Design based on linear model}

The characteristic equation of a compensated linear system with controller $\mathbf{u}=-K \mathbf{x}$ is $|s \mathbf{I}-\mathbf{A}+\mathbf{B} K|=0$. If the system is in the phase-variable canonical form then the characteristic equation of the compensated linear system is

$$
|s \mathbf{I}-\mathbf{A}+\mathbf{B} K|=s^{n}+\left(a_{n-1}+k_{n}\right)+\cdots+\left(a_{1}+k_{2}\right) s+\left(a_{0}+k_{1}\right)=0
$$

where $a_{n-1}, a_{n-2} \cdots a_{1}$, and $a_{0}$ are the coefficients of the characteristic equation $|s \mathbf{I}-\mathbf{A}|=0$ and $k_{n} \cdots$ $k_{1}$, are gains of the control matrix $K$. For the specified closed-loop pole locations $-\lambda_{1}, \cdots-\lambda_{n}$ the desired characteristic equation is

$$
\alpha_{c}(s)=\left(s+\lambda_{1}\right)\left(s+\lambda_{2}\right) \cdots\left(s+\lambda_{n}\right)=s^{n}+\alpha_{n-1} s^{n-1}+\cdots+\alpha_{1} s+\alpha_{0}=0
$$

The design objective is to find the gain matrix $K$ such that the characteristic equation for the controlled system is identical to the desired characteristic equation. Thus, the gain vector $K$ is obtained by equating coefficients of Equation (7.12) and Equation (7.13) and for the $i^{\text {th }}$ coefficient we get

$$
k_{i}=\alpha_{i}-a_{i}
$$

If the state model is not in the phase-variable canonical form, we can use the transformation technique to transform the given state model to the phase-variable canonical form which results in the following formula, known as Ackermann's formula [8].

$$
K=\left[\begin{array}{lllll}
0 & 0 & \cdots & 0 & 1
\end{array}\right] S^{-1} \alpha_{c}(A)
$$

where the matrix $S$ is given by

$$
S=\left[\begin{array}{lllll}
B & A B & A^{2} B & \cdots & A^{n-1} B
\end{array}\right]
$$

and $\alpha_{c}(A)$ is given by

$$
\alpha_{c}(A)=A^{n}+\alpha_{n-1} A^{n-1}+\cdots+\alpha_{1} A+\alpha_{0} I
$$

The MATLAB function $K=\operatorname{place}(A, B, p)$ can be used to design the controller gain matrix $K$, where $p$ is a row vector containing the desired closed-loop poles. The closed-loop poles are selected such that all the state variables remain within a specific limit and converge to zero in minimum time. The closed-loop poles cannot be placed too close to the imaginary axis as the relative stability of the system decreases and the system oscillations increase. Also if they are placed far away from the imaginary axis the settling time of the state variables and hence the output decreases, but there is no guarantee that the state variables and the control inputs will remain within a reasonable physical limit. After some trial and error the desired closed-loop poles of the generator-turbine system were selected as

$$
p=[-0.8,-0.9,-0.7,-1.1,-1]
$$

The controller gain matrix $K$ corresponding to these closed loop poles is

$$
K=\left[\begin{array}{ccccc}
12.6444 & -44.1610 & 0.9926 & -3.1472 & -8.9082 \\
0.0535 & -0.3867 & -0.0861 & 0.0788 & -1.0390
\end{array}\right]
$$

Figure 7.20 to Figure 7.23 show that the state variables $\Delta E_{q}^{\prime}, \Delta \omega, \Delta \delta, \Delta T_{m}, \Delta G_{V}$, and the outputs $\Delta V_{t}, \Delta \omega$ and $\Delta \delta$ settle to their steady state value of zero in approximately 10 seconds. Figure 7.24 and Figure 7.25 show plots for the two control inputs $\Delta E_{f d}$, and $\Delta u_{T}$ respectively. 


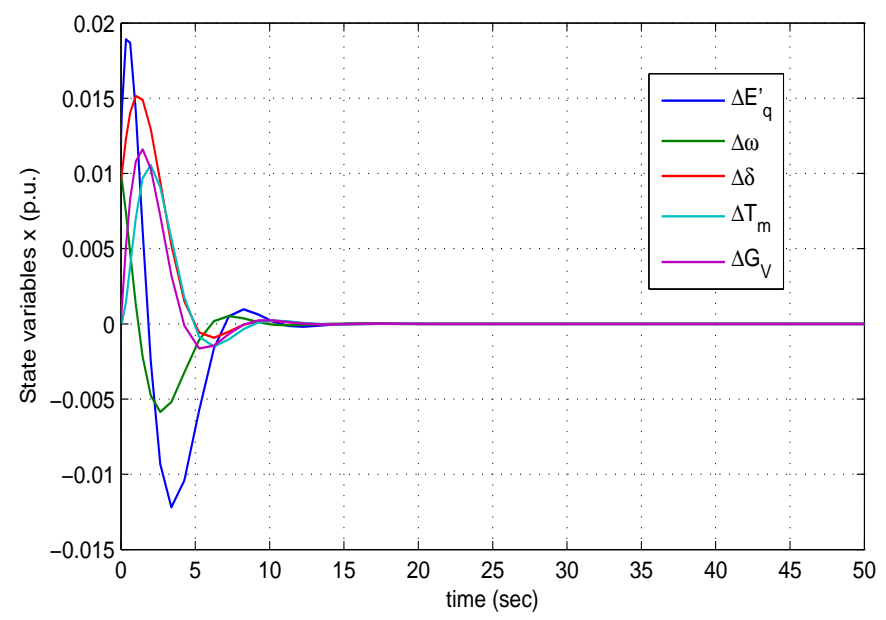

Figure 7.20: Plot of the state variables $\Delta E_{q}^{\prime}, \Delta \omega, \Delta \delta, \Delta T_{m}$, and $\Delta G_{V}$ vs time for the pole placement based full-state feedback controller applied to the reduced order linear model

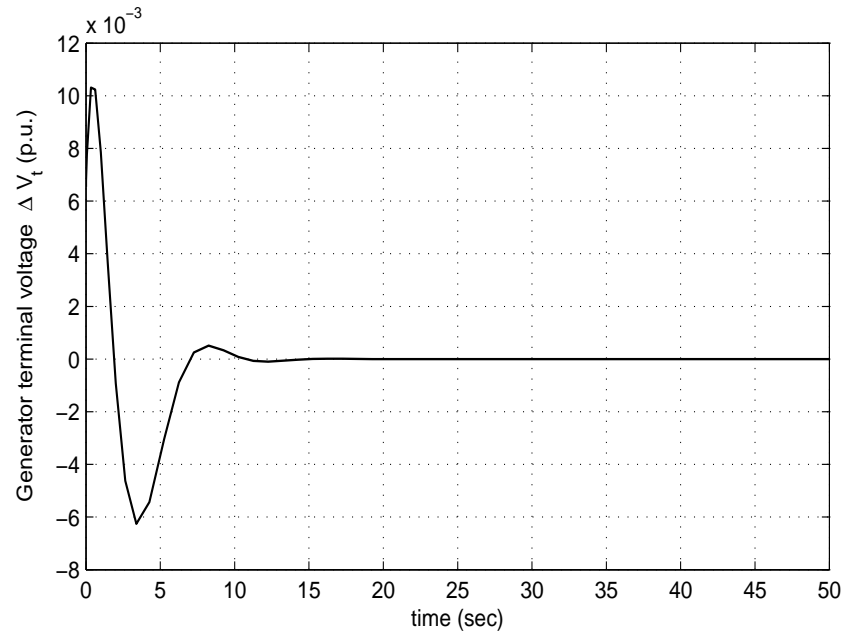

Figure 7.21: Plot of the generator terminal voltage $\Delta V_{t}$ vs time for the pole placement based full-state feedback controller applied to the reduced order linear model

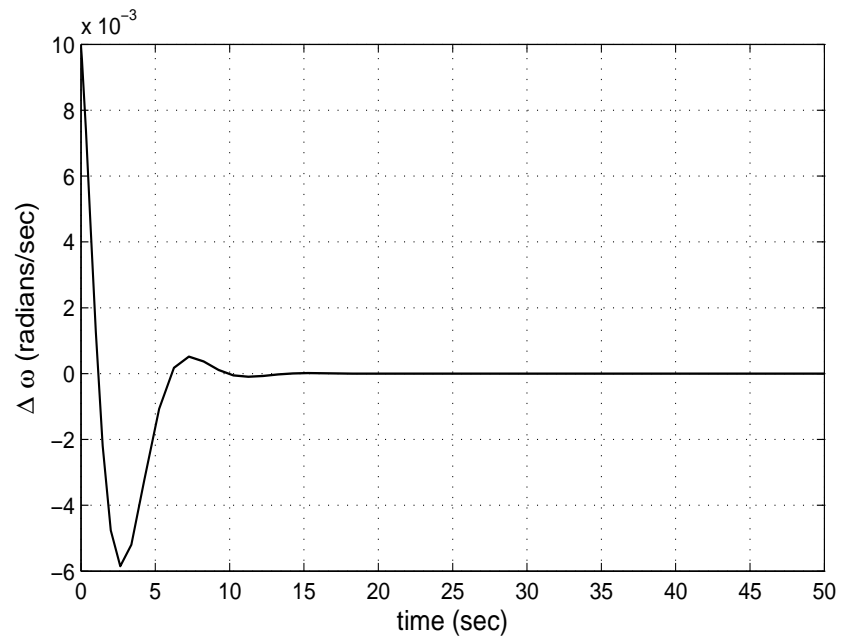

Figure 7.22: Plot of $\Delta \omega$ vs time for the pole placement based full-state feedback controller applied to the reduced order linear model 


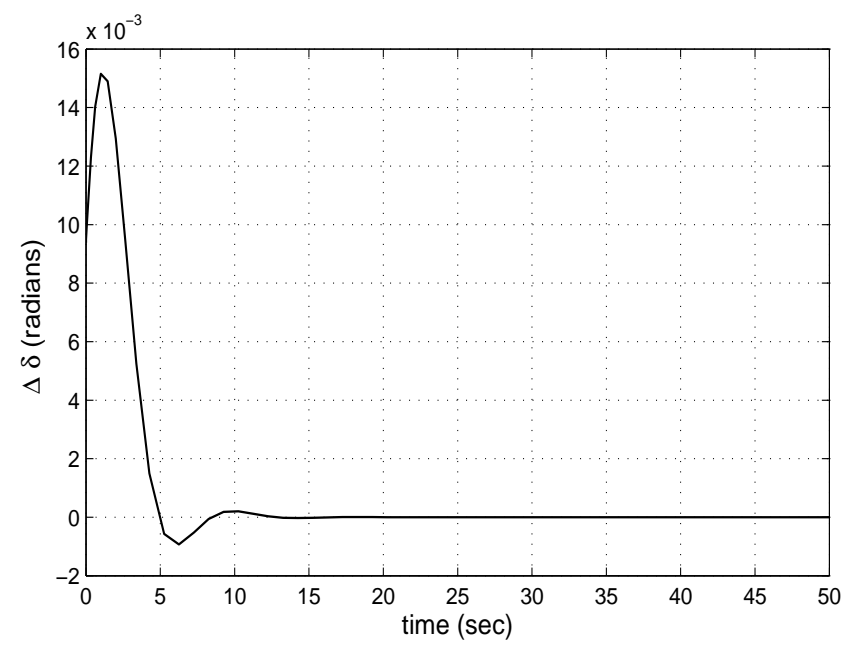

Figure 7.23: Plot of $\Delta \delta$ vs time for the pole placement based full-state feedback controller applied to the reduced order linear model

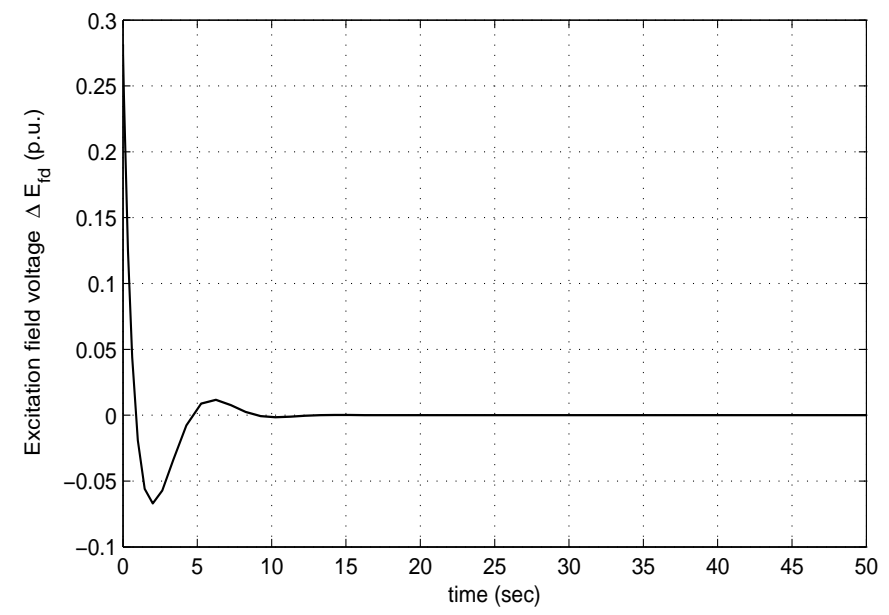

Figure 7.24: Plot of the control input $\Delta E_{f d}$ vs time for the pole placement based full-state feedback controller applied to the reduced order linear model

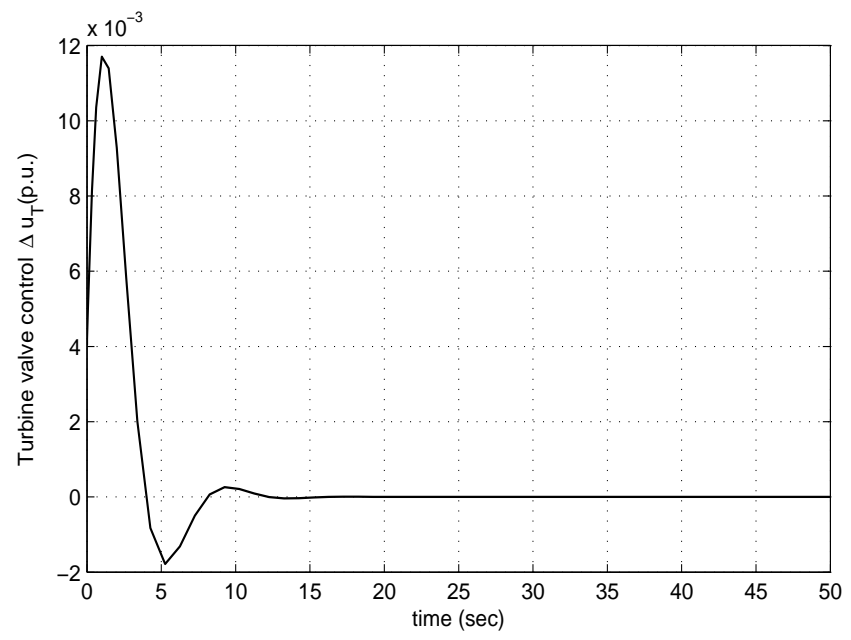

Figure 7.25: Plot of the control input $\Delta u_{T}$ vs time for the pole placement based full-state feedback controller applied to the reduced order linear model 


\subsubsection{Simulation Results for the Full-State Feedback Pole Placement controller applied to the Reduced Order Nonlinear Model}

We now test the pole placement based full-state feedback controller that was designed earlier for the reduced order linear model, on the reduced order nonlinear model. Figure 7.26 and Figure 7.27 show plots for the generator terminal voltage $V_{t}$, and rotor angle $\delta$, respectively. The generator terminal voltage $V_{t}$ settles to a new steady state value of 0.8116 p.u. which deviates from the desired steady state value of 1.1723 p.u. by an amount of $0.3607 \mathrm{p} . \mathrm{u}$. Similarly the rotor angle $\delta$ settles to a new steady state value of 1.324 p.u. which deviates from the desired steady state value of $1 \mathrm{p} . \mathrm{u}$. by an amount of $0.324 \mathrm{p} . \mathrm{u}$. We observe a large steady state error when the pole placement controller with original gains of subsection 7.2.1 is applied to the reduced order nonlinear model. Therefore, the gains of the controller are once again tuned by appropriately choosing the desired pole locations, to get satisfactory performance. The MATLAB function $K=\operatorname{place}(A, B, p)$ can be used to design the controller gain matrix $K$, where $p$ is a row vector containing the desired closed-loop poles. After some trial and error the desired closed-loop poles of the generator-turbine system were selected as

$$
p=[-300,-0.9,-280,-5,-70]
$$

We see here that poles $\mathrm{p}(1), \mathrm{p}(3)$, and $\mathrm{p}(5)$ are placed far away from the imaginary axis so that the state variables $E_{q}^{\prime}, \delta$, and $G_{V}$ attain their desired steady state values in less time. At the same time care is taken that these state variables stay within a safe physical limit. The controller gain matrix $K$ corresponding to these closed loop poles is

$$
K=\left[\begin{array}{ccccc}
2020 & -376860 & -304950 & -18250 & -130 \\
-10 & 4840 & 3770 & 330 & 60
\end{array}\right]
$$

Figure 7.28-Figure 7.31 show that the state variables $E_{q}^{\prime}, \omega, \delta, T_{m}, G_{V}$, and the outputs $V_{t}$ and $\delta$ settle to their respective steady state values in approximately 5 to 8 seconds. 


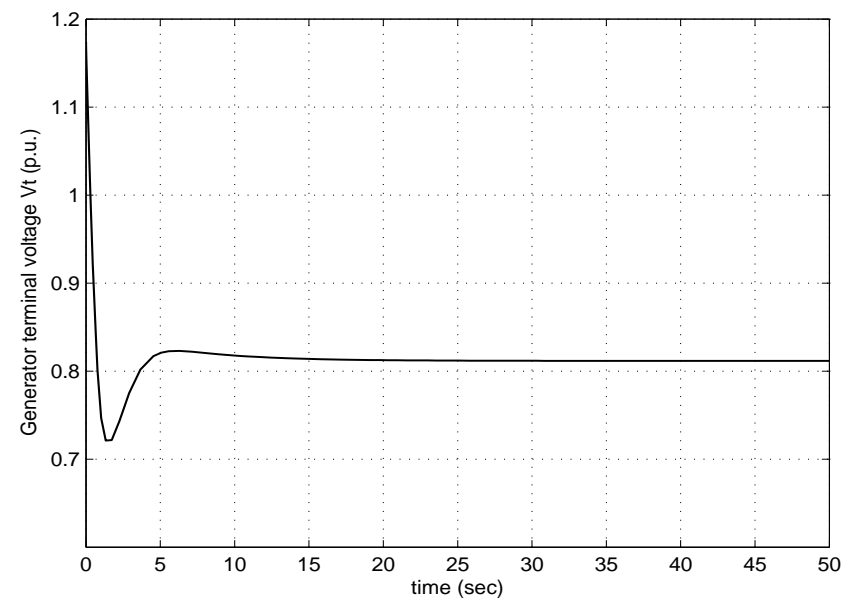

Figure 7.26: Plot of the generator terminal voltage $V_{t}$ vs time for the pole placement based full-state feedback controller with the original gains of subsection 7.2.1 applied to the reduced order nonlinear model

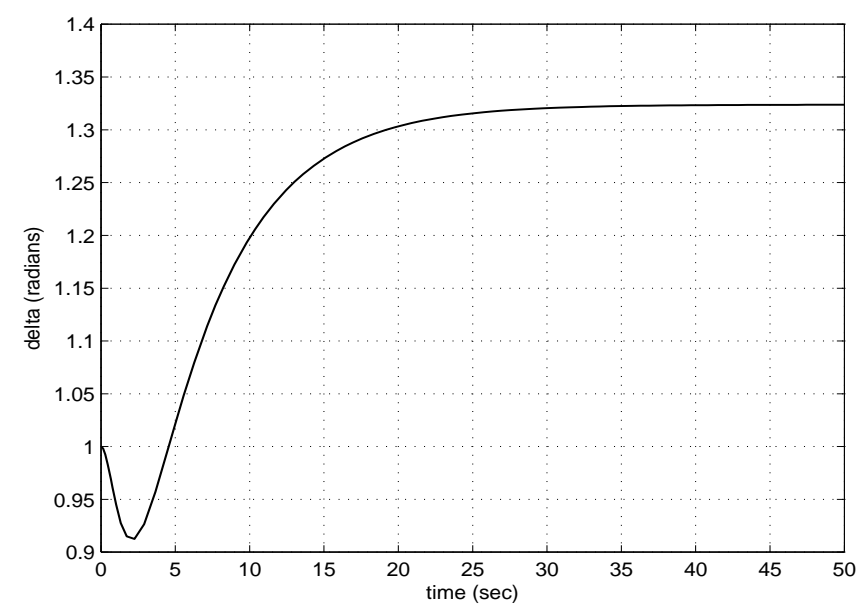

Figure 7.27: Plot of the rotor angle $\delta$ vs time for the pole placement based full-state feedback controller with the original gains of subsection 7.2.1 applied to the reduced order nonlinear model

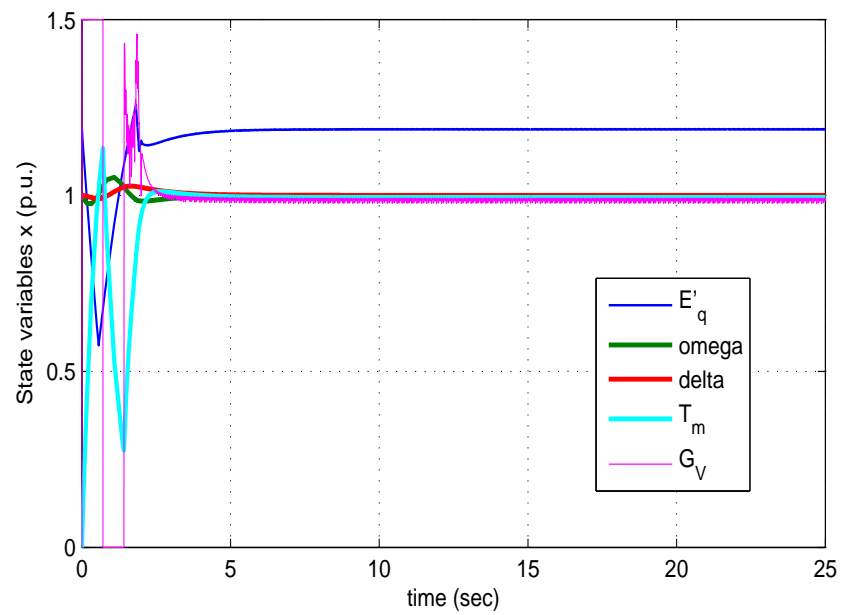

Figure 7.28: Plot of the state variables $E_{q}^{\prime}, \omega, \delta, T_{m}$, and $G_{V}$ vs time for the pole placement based full-state feedback controller applied to the reduced order nonlinear model, with the gains re-tuned 


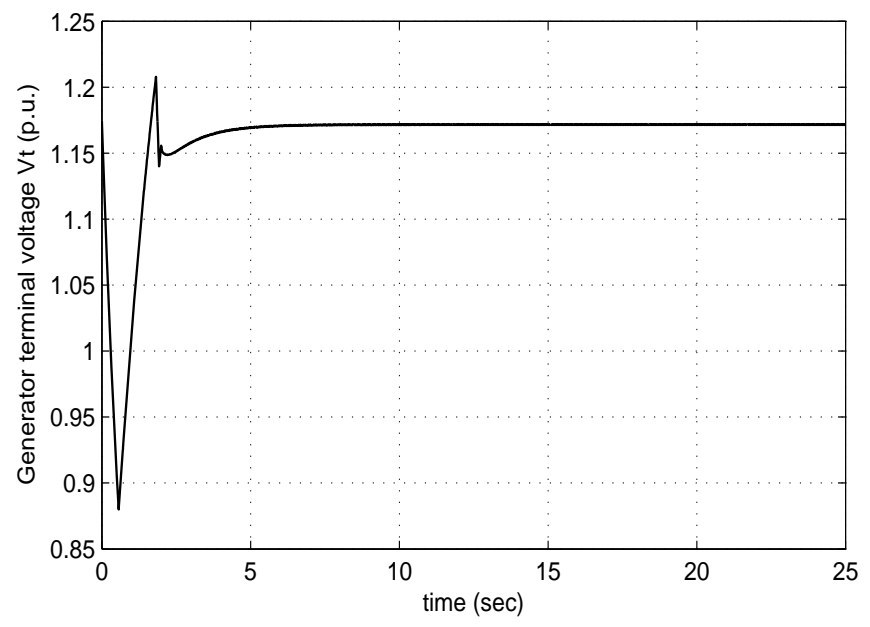

Figure 7.29: Plot of the generator terminal voltage $V_{t}$ vs time for the pole placement based full-state feedback controller applied to the reduced order nonlinear model, with the gains re-tuned

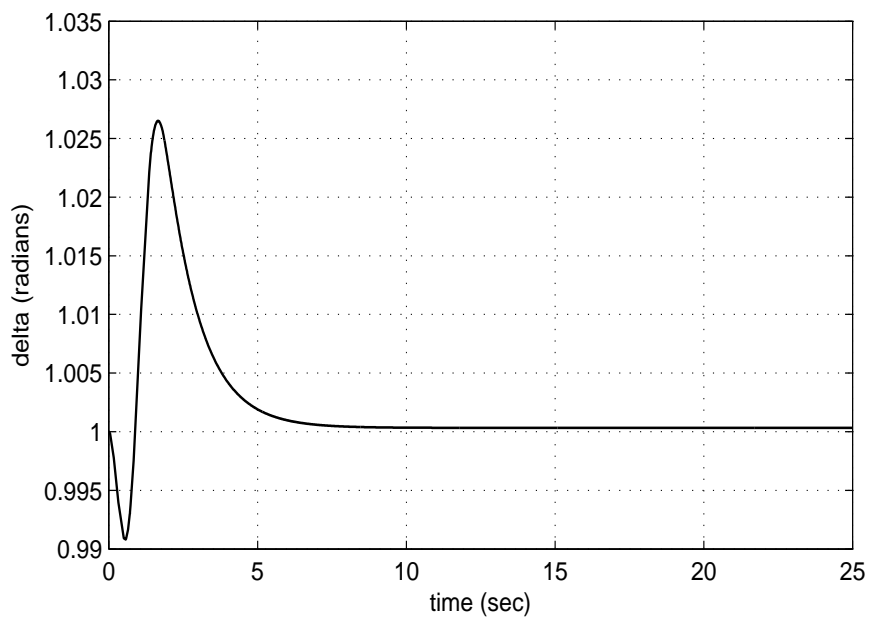

Figure 7.30: Plot of the rotor angle $\delta$ vs time for the pole placement based full-state feedback controller applied to the reduced order nonlinear model, with the gains re-tuned

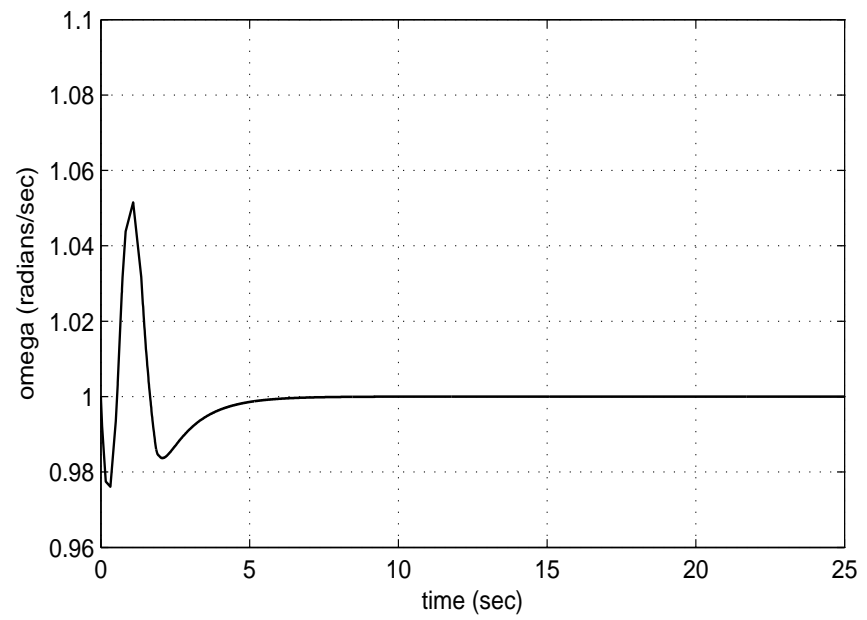

Figure 7.31: Plot of the frequency $\omega$ vs time for the pole placement based full-state feedback controller applied to the reduced order nonlinear model, with the gains re-tuned 


\subsubsection{Simulation Results for the Full-State Feedback Pole Placement controller applied to the Truth Model}

We now test the pole placement based full-state feedback controller on the truth model. The gains of the controller are re-tuned by appropriately choosing the desired pole locations, to get satisfactory performance. The MATLAB function $K=\operatorname{place}(A, B, p)$ is used to design the controller gain matrix $K$, where $p$ is a row vector containing the desired closed-loop poles. After some trial and error the desired closed-loop poles of the generator-turbine system were selected as

$$
p=[-8.00+j 0.05,-8.00-j 0.05,-200,-250,-0.1]
$$

The controller gain matrix $K$ corresponding to these closed loop poles is

$$
K=\left[\begin{array}{ccccc}
1526.38 & -48186.24 & -3062.64 & -1166.75 & -32.7 \\
-0.9566 & 1321.51 & 49.346 & 103.52 & 39.965
\end{array}\right]
$$

Figure 7.32 to Figure 7.36 show simulation results for the pole placement based full-state feedback controller applied to the truth model. From these results we can see that $V_{t}$ oscillates about a steady state value of 1.17 p.u. which deviates from the desired steady state value of 1.1723 p.u. by an amount of 0.0023 p.u., $\omega$ oscillates about its desired steady state value of 1 p.u., and $\delta$ oscillates about a steady state value of 1.0005 p.u. which deviates from the desired steady state value of 1 p.u. by an amount of 0.0005 p.u.. Small oscillations which decay with time are seen for $\omega$, and $\delta$. Figure 7.35 and Figure 7.36 show plots for the two control inputs $V_{F}$ and $u_{T}$ respectively. As seen from these plots, the generator excitation voltage $V_{F}$ settles to its steady state value of 0.00121 p.u. and the turbine valve control settles to its steady state value of 1.0512 p.u. 


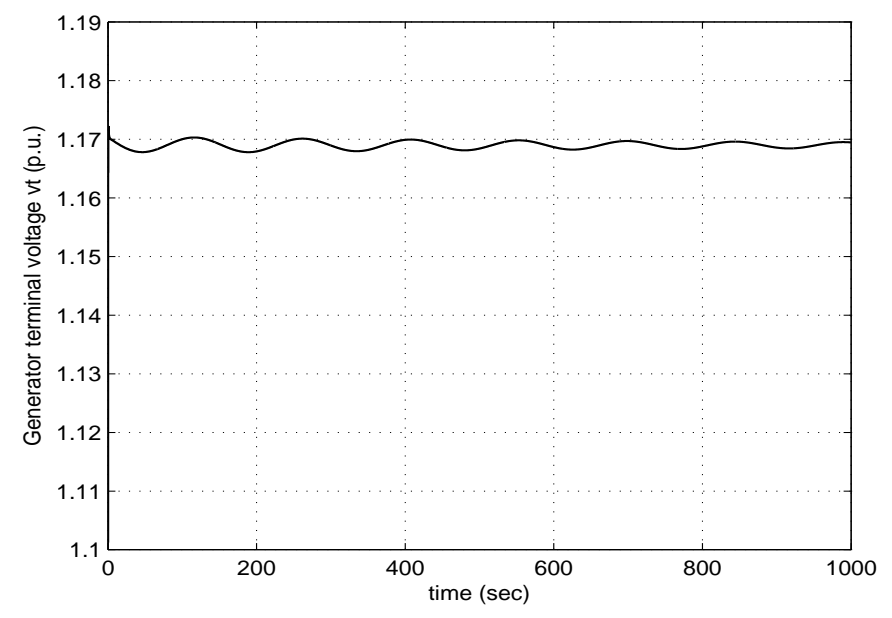

Figure 7.32: Plot of the generator terminal voltage $V_{t}$ vs time for the pole placement based full-state feedback controller applied to the truth model

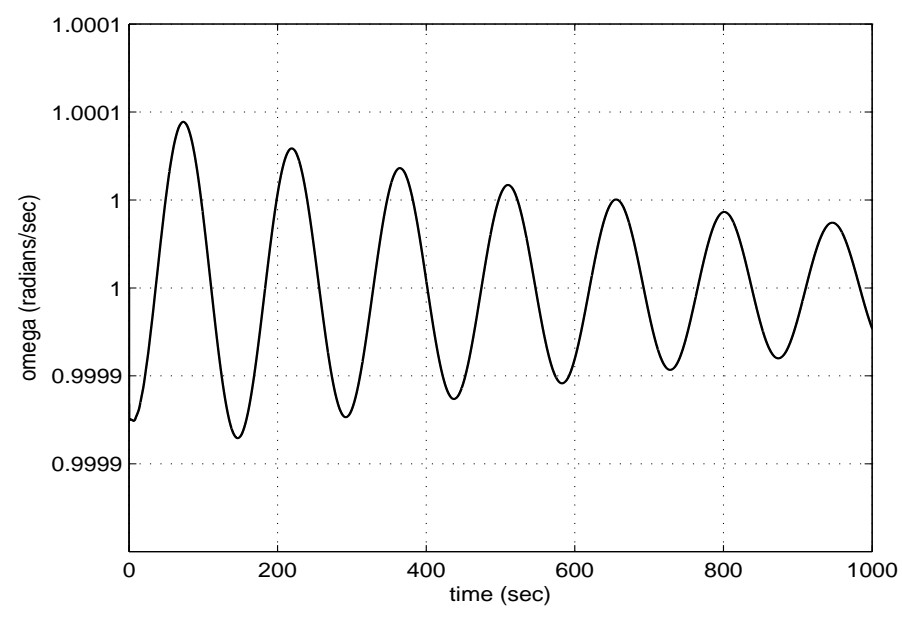

Figure 7.33: Plot of $\omega$ vs time for the pole placement based full-state feedback controller applied to the truth model

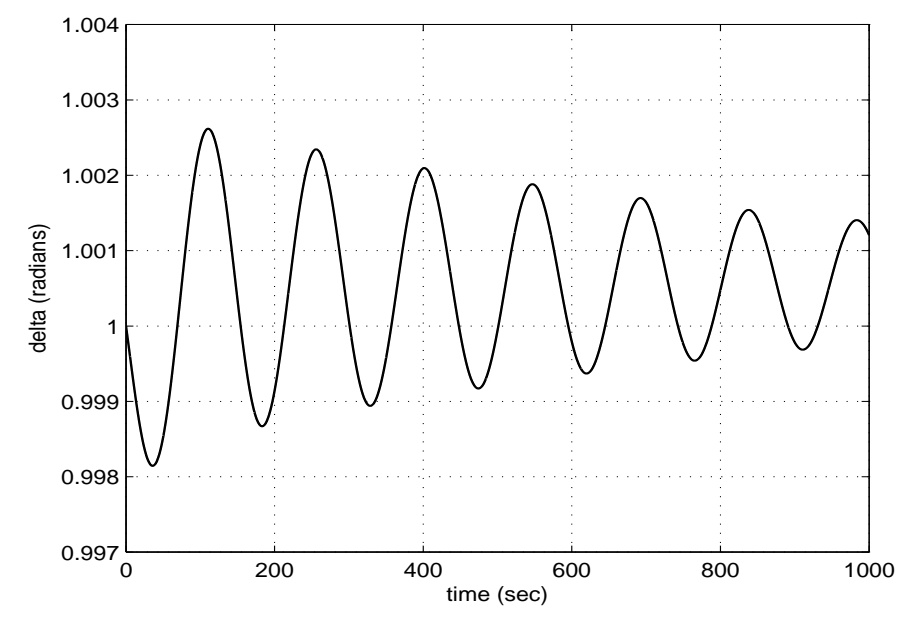

Figure 7.34: Plot of $\delta$ vs time for the pole placement based full-state feedback controller applied to the truth model 


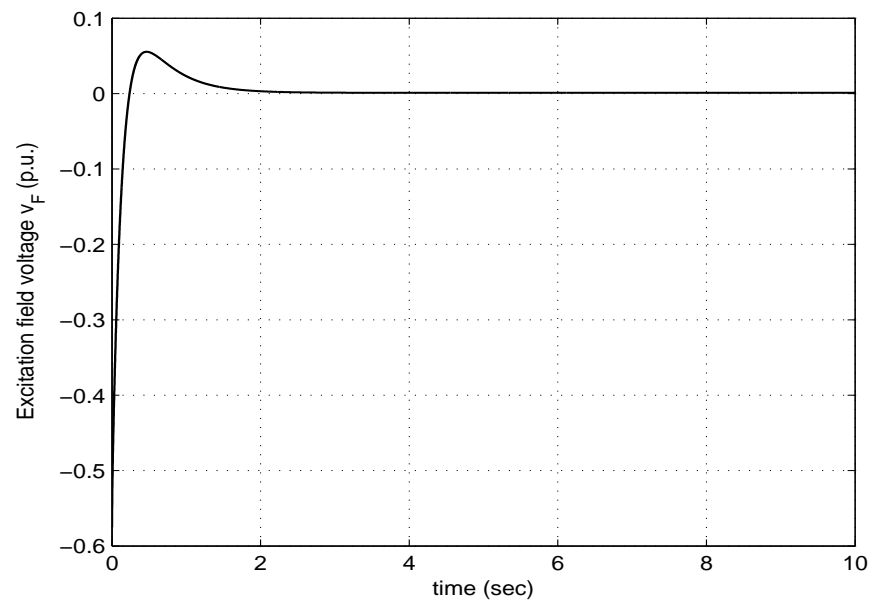

Figure 7.35: Plot of the control input $V_{F}$ vs time for the pole placement based full-state feedback controller applied to the truth model

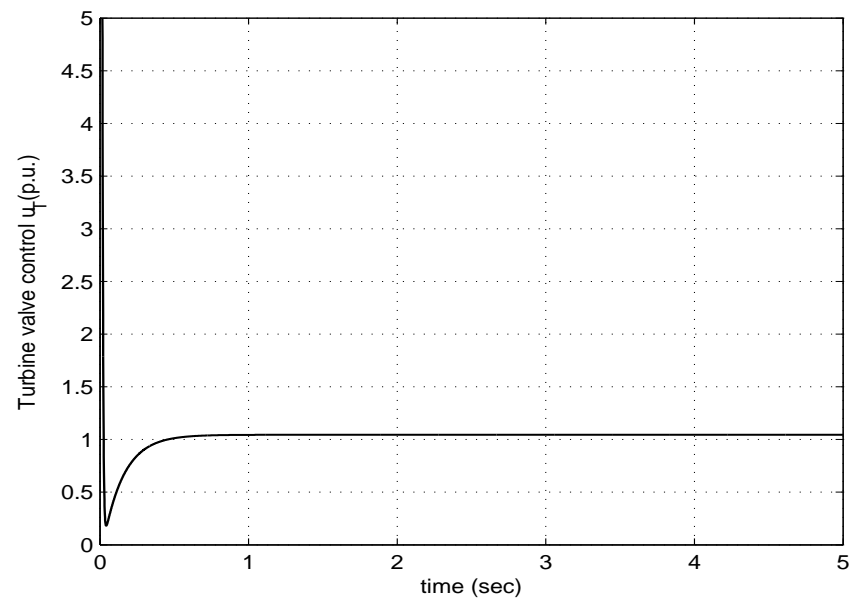

Figure 7.36: Plot of the control input $u_{T}$ vs time for the pole placement based full-state feedback controller applied to the truth model 


\subsection{Output Feedback Controller Design}

\subsubsection{Observer-based LQR Design based on linear model}

In a practical generator-turbine system connected to an infinite bus not all the states are available for measurement. For example the second output of the system which is the rotor angle cannot be measured using a sensor. Thus, we need to design an observer to estimate the rotor angle and the remaining unmeasured states. Once the estimator is designed, using the separation principle state estimates are used as the input to the state feedback controller to obtain a regulator for the system. We first use only one sensor to measure only the first output $\Delta V_{t}$. Later we use two sensors to measure both the outputs $\Delta V_{t}$, and $\Delta \omega$. Using the $A$ and $B$ matrices as found earlier and

$$
\begin{aligned}
\mathbf{y} & =C_{1} \mathbf{x}=\left[\begin{array}{lllll}
T_{1} & 0 & T_{2} & 0 & 0
\end{array}\right] \mathbf{x} \\
\text { i.e. } \Delta V_{t} & =\left[\begin{array}{lllll}
0.5258 & 0 & 0.0294 & 0 & 0
\end{array}\right] \mathbf{x}
\end{aligned}
$$

where we use only the first output i.e. the generator terminal voltage $\Delta V_{t}$ measurement for the observer design. Using MATLAB we can verify that the system is observable using the generator terminal voltage $\Delta V_{t}$ i.e. $C_{1}$ as given in Equation (7.24). The observer that produces an estimate of the state, $\hat{x}$, is of the form

$$
\begin{aligned}
\dot{\hat{x}} & =A \hat{x}+B u+L(y-\hat{y}) \\
\hat{y} & =C_{1} \hat{x}
\end{aligned}
$$

The observer gain matrix $L$ in the above equation is designed such that the error dynamics of the estimator decay faster than the remaining dynamics. Let us denote $e=x-\hat{x}$ as the estimation error. Then, the error dynamics of the estimator are given by

$$
\dot{e}=\left(A-L C_{1}\right) e
$$

The procedure to design the observer gain matrix $L$ is as follows: We first calculate the eigenvalues of the closed loop system, i.e. eigenvalues of the closed loop $A c p$ matrix where $A c p=A-B K$. The real parts of the estimator poles are selected 10-15 times to the left of the closed loop poles of the system Acp matrix, so that the error dynamics of the estimator decay to zero faster. This can be achieved by appropriately selecting the scaling factor $\rho$ which places the estimator poles to the left of the closed loop poles of the system. The following MATLAB command can be used to design the observer gain matrix

poles $=\operatorname{eig}(A c p)$

$p=r h o *[\operatorname{poles}(1), \operatorname{poles}(2), \operatorname{poles}(3), \operatorname{poles}(4), \operatorname{poles}(5)]$;

Ltilde $=\operatorname{place}\left(A^{\prime}, C_{1}^{\prime}, p\right)$;

$L=$ Ltilde'

The estimator gain matrix $L$ for $\rho=12$ is

$$
L=\left[\begin{array}{c}
1510.4 \\
-65508.0 \\
-24107.6 \\
-1004232.6 \\
276190.3
\end{array}\right]
$$

Next, the estimated states are fed back to the feedback controller to obtain a regulator for the system

$$
u=-K \hat{x}
$$

Choosing the weighting matrices

$$
Q=\left[\begin{array}{lllll}
1 & 0 & 0 & 0 & 0 \\
0 & 1 & 0 & 0 & 0 \\
0 & 0 & 1 & 0 & 0 \\
0 & 0 & 0 & 1 & 0 \\
0 & 0 & 0 & 0 & 1
\end{array}\right]
$$


and

$$
R=\left[\begin{array}{cc}
20 & 0 \\
0 & 20
\end{array}\right]
$$

and the state space matrices $(A, B)$ as given in Equation (3.76) and Equation (3.77) the control gain $K$ for the observer-based LQR with the output $V_{t}$ measured is found to be

$$
K=\left[\begin{array}{ccccc}
0.0384 & -0.0635 & 0.0126 & -0.0070 & -0.0027 \\
-0.0811 & 0.1973 & -0.0236 & 0.0382 & 0.0395
\end{array}\right]
$$

The $Q$ and $R$ matrices are designed such that all the estimated state variables and the control signals stay within a specific limit. From the choice of the $Q$ and $R$ matrices for the estimator-based LQR with output $\Delta V_{t}$ measured, we can see that the weights of the $Q$ matrix are reduced and weights of the $R$ matrix are increased compared to the full state feedback LQR. In other words the amount of control energy that can be provided such that all the state variables and control inputs remain within a limit is significantly reduced. This is allowed by the separation principle where the controller and the observer gains are designed independently. Thus, the time taken by the estimated states and hence the outputs to reach the steady state value of 0 is larger than the state feedback case. This is evident from Figure 7.37 to Figure 7.40 where the estimated states $\Delta \hat{E}^{\prime}{ }_{q}, \Delta \hat{\omega}, \Delta \hat{\delta}, \Delta \hat{T}_{m}, \Delta \hat{G}_{V}$, and the outputs $\Delta V_{t}, \Delta \omega$, and $\Delta \delta$ take more time to converge to zero as compared to the LQR with full state feedback. Figure 7.41 and Figure 7.42 show plots for the two control inputs $\Delta E_{f d}$, and $\Delta u_{T}$ respectively.

A better estimate of the states can be obtained by also using the angular velocity or frequency $\Delta \omega$ of the synchronous generator which can be measured using a sensor, in addition to the generator terminal voltage $\Delta V_{t}$. In this case we have

$$
\begin{aligned}
\mathbf{y} & =C_{2} \mathbf{x}=\left[\begin{array}{ccccc}
T_{1} & 0 & T_{2} & 0 & 0 \\
0 & 1 & 0 & 0 & 0
\end{array}\right] \mathbf{x} \\
\text { i.e. }\left[\begin{array}{c}
\Delta V_{t} \\
\Delta \omega
\end{array}\right] & =\left[\begin{array}{ccccc}
0.5258 & 0 & 0.0294 & 0 & 0 \\
0 & 1 & 0 & 0 & 0
\end{array}\right] \mathbf{x}
\end{aligned}
$$

Using MATLAB we can verify that the system is observable using the generator terminal voltage $\Delta V_{t}$ and the frequency $\Delta \omega$ i.e. $C_{2}$ as given in Equation (7.32). The estimator gain matrix $L$ for the output matrix $C_{2}$ as given in Equation (7.32) and $\rho=12$ is

$$
L=\left[\begin{array}{cc}
256.459 & 71.2024 \\
20.6146 & 34.7633 \\
-1846.26 & -922.386 \\
-5032.595 & -1309.90 \\
-531.7120 & -135.4256
\end{array}\right]
$$

Choosing the weighting matrices

$$
Q=\left[\begin{array}{lllll}
1 & 0 & 0 & 0 & 0 \\
0 & 1 & 0 & 0 & 0 \\
0 & 0 & 1 & 0 & 0 \\
0 & 0 & 0 & 1 & 0 \\
0 & 0 & 0 & 0 & 1
\end{array}\right]
$$

and

$$
R=\left[\begin{array}{ll}
1 & 0 \\
0 & 1
\end{array}\right]
$$

and the state space matrices $(A, B)$ as given in Equation (3.76) and Equation (3.77) the control gain $K$ for the observer-based LQR with the outputs $\Delta V_{t}$ and $\Delta \omega$ measured is found to be

$$
K=\left[\begin{array}{ccccc}
0.4722 & -0.8024 & 0.0599 & -0.0726 & -0.0195 \\
-0.5758 & 1.6563 & -0.0271 & 0.3948 & 0.5217
\end{array}\right]
$$




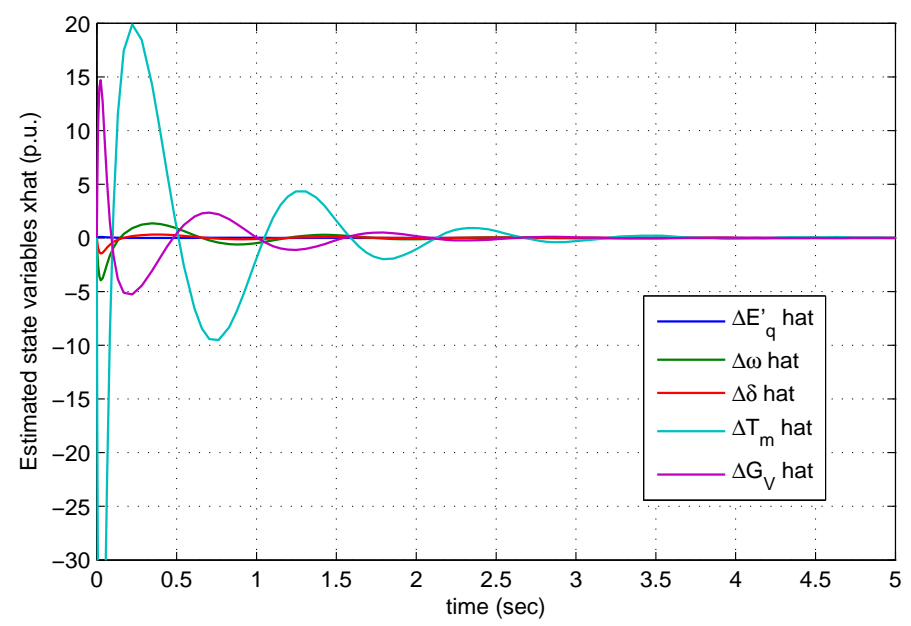

Figure 7.37: Plot of the estimated state variables $\Delta \hat{E}_{q}^{\prime}, \Delta \hat{\omega}, \Delta \hat{\delta}, \Delta \hat{T}_{m}$, and $\Delta \hat{G}_{V}$ vs time for the observerbased LQR applied to the reduced order linear model with output $\Delta V_{t}$ measured

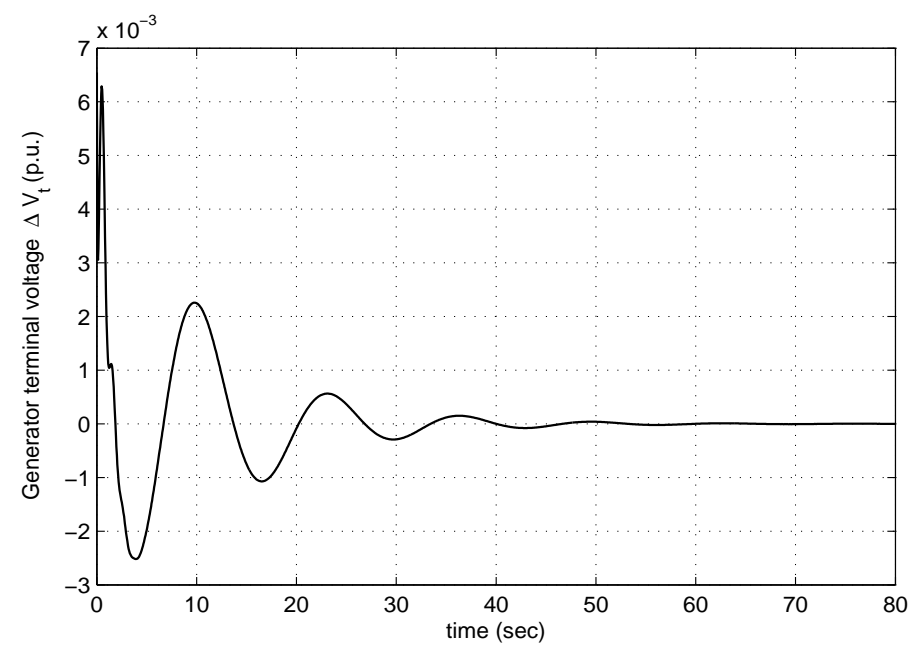

Figure 7.38: Plot of the generator terminal voltage $\Delta V_{t}$ vs time for the observer-based LQR applied to the reduced order linear model with output $\Delta V_{t}$ measured

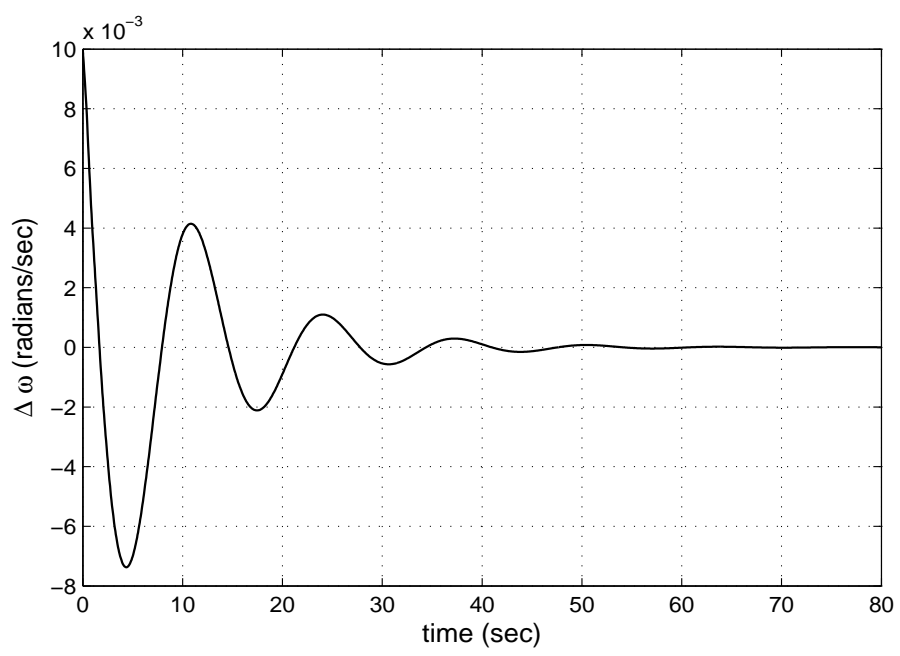

Figure 7.39: Plot of $\Delta \omega$ vs time for the observer-based LQR applied to the reduced order linear model with output $\Delta V_{t}$ measured 


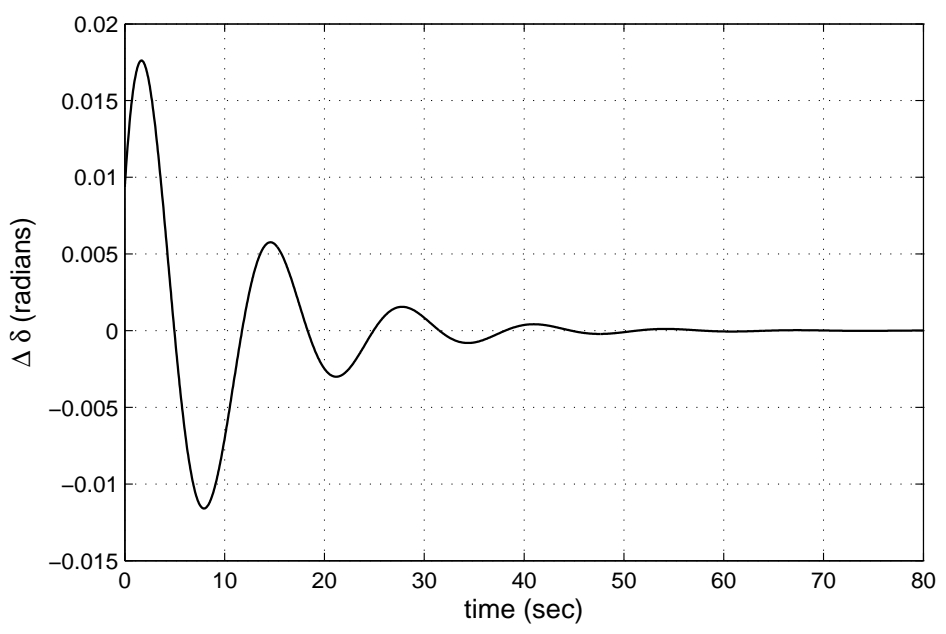

Figure 7.40: Plot of the rotor angle $\Delta \delta$ vs time for the observer-based LQR applied to the reduced order linear model with output $\Delta V_{t}$ measured

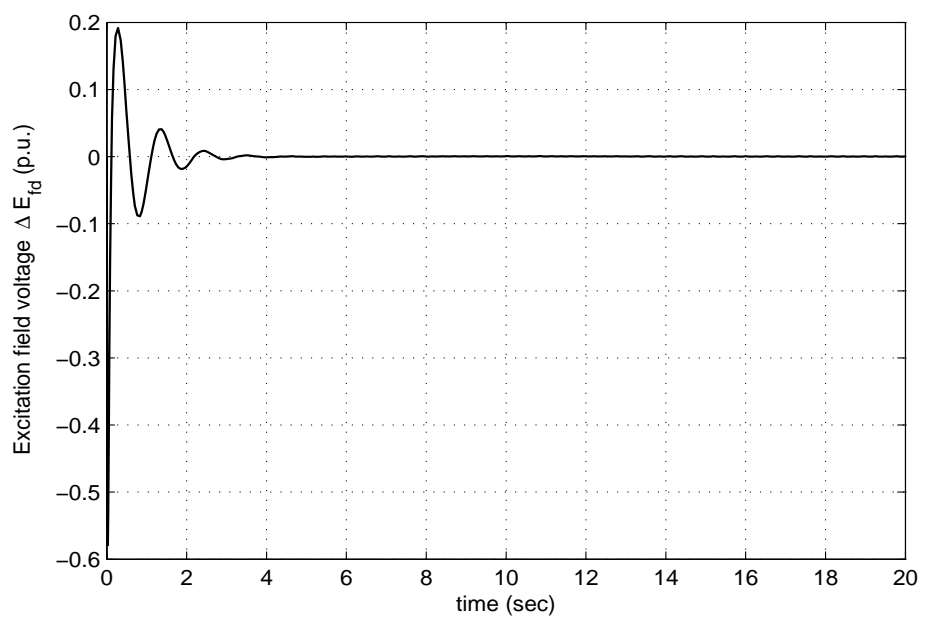

Figure 7.41: Plot of the control input $\Delta E_{f d}$ vs time for the observer-based LQR applied to the reduced order linear model with output $\Delta V_{t}$ measured

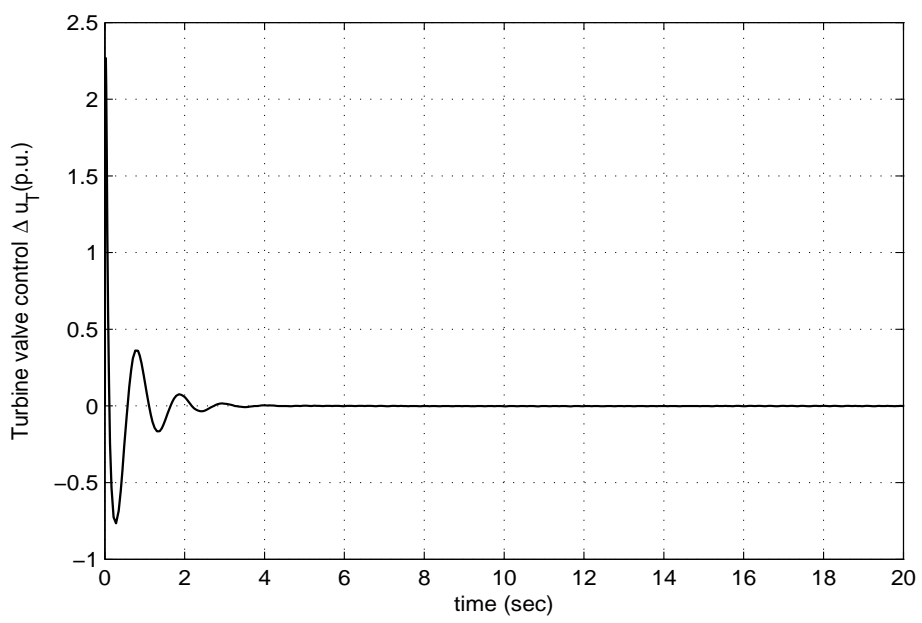

Figure 7.42: Plot of the control input $\Delta u_{T}$ vs time for the observer-based LQR applied to the reduced order linear model with output $\Delta V_{t}$ measured 


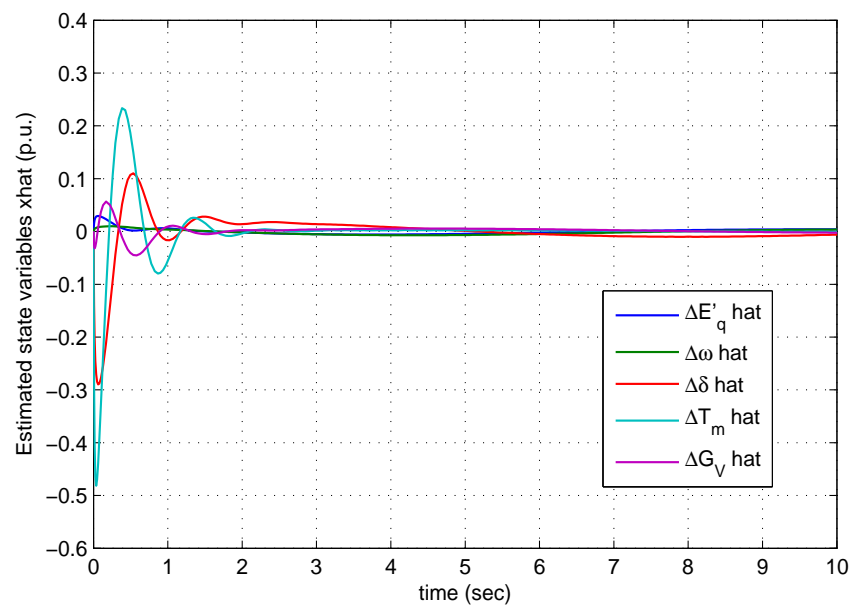

Figure 7.43: Plot of the estimated state variables $\Delta \hat{E}^{\prime}{ }_{q}, \Delta \hat{\omega}, \Delta \hat{\delta}, \Delta \hat{T}_{m}$, and $\Delta \hat{G}_{V}$ vs time for the observerbased LQR applied to the reduced order linear model with outputs $\Delta V_{t}$ and $\Delta \omega$ measured

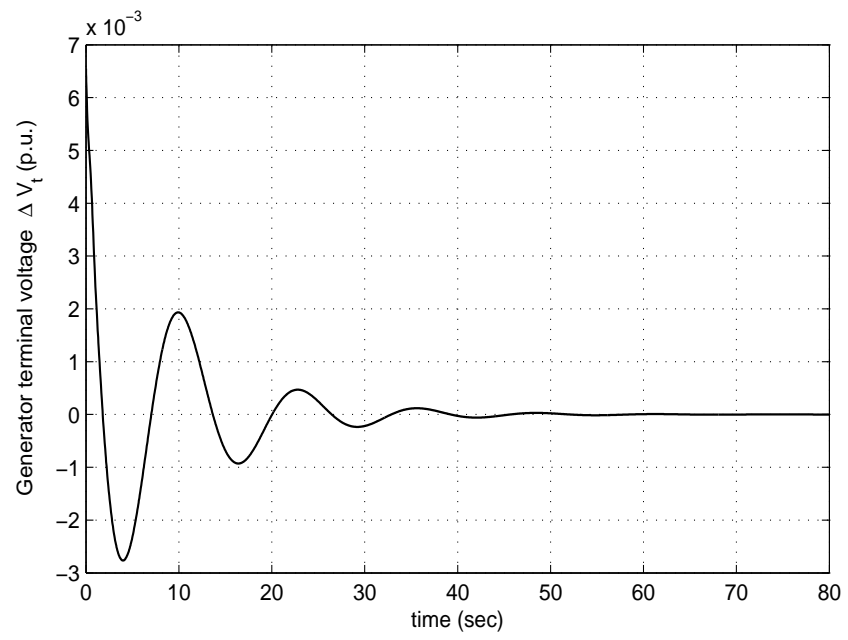

Figure 7.44: Plot of the generator terminal voltage $\Delta V_{t}$ vs time for the observer-based LQR applied to the reduced order linear model with outputs $\Delta V_{t}$ and $\Delta \omega$ measured

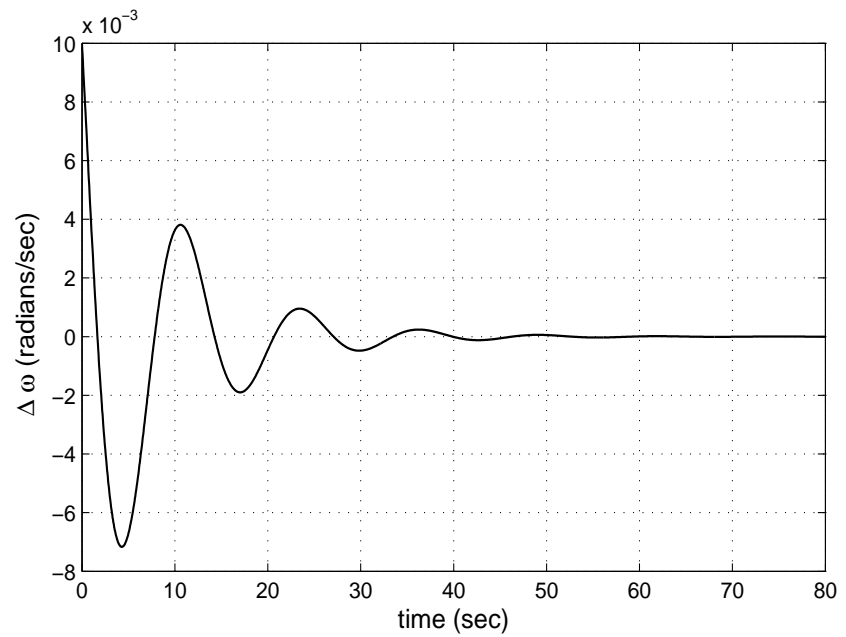

Figure 7.45: Plot of $\Delta \omega$ vs time for the observer-based LQR applied to the reduced order linear model with outputs $\Delta V_{t}$ and $\Delta \omega$ measured 


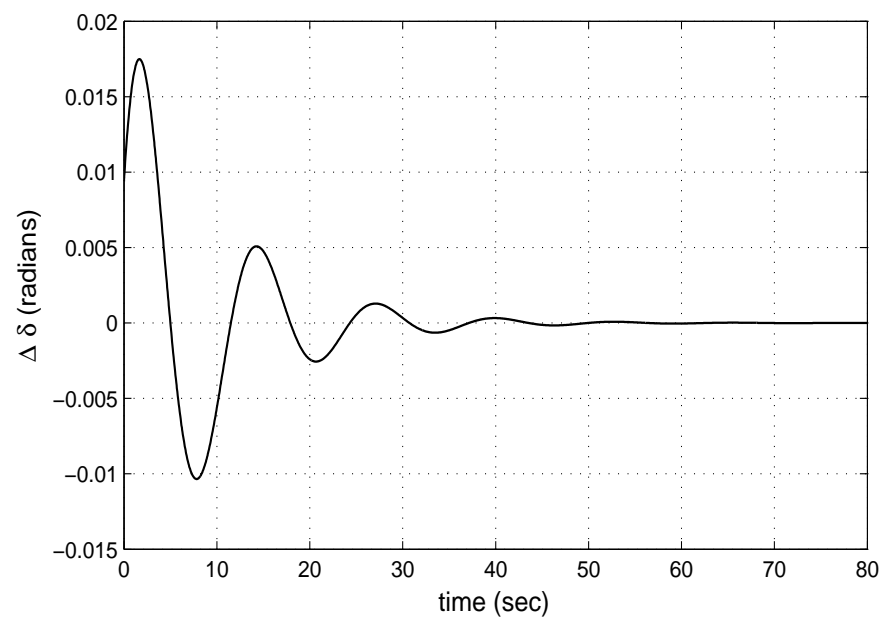

Figure 7.46: Plot of $\Delta \delta$ vs time for the observer-based LQR applied to the reduced order linear model with outputs $\Delta V_{t}$ and $\Delta \omega$ measured

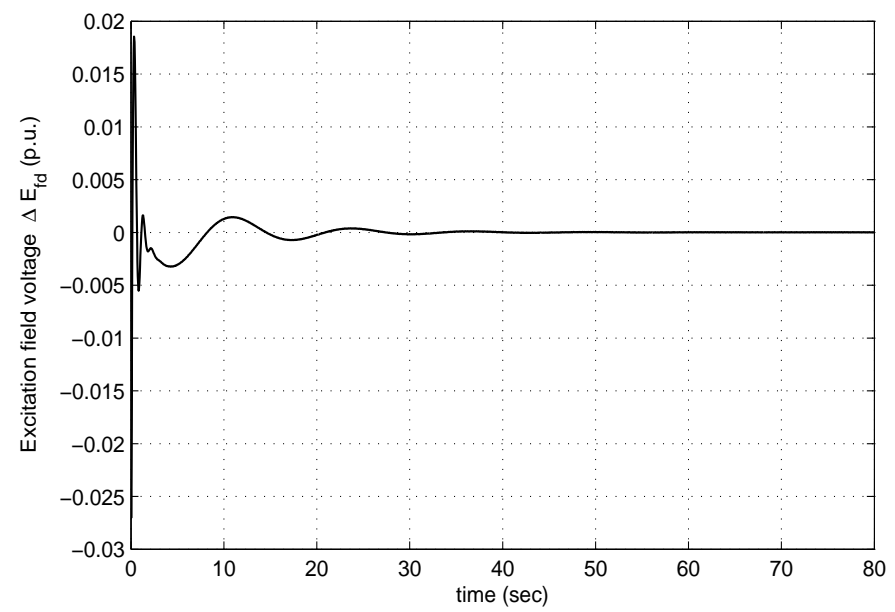

Figure 7.47: Plot of the control input $\Delta E_{f d}$ vs time for the observer-based LQR applied to the reduced order linear model with outputs $\Delta V_{t}$ and $\Delta \omega$ measured

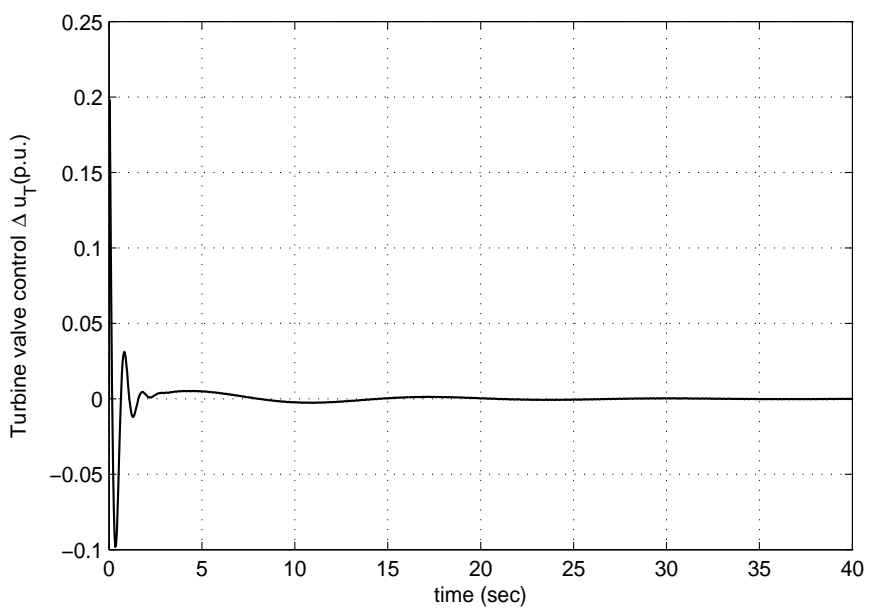

Figure 7.48: Plot of the control input $\Delta u_{T}$ vs time for the observer-based LQR applied to the reduced order linear model with outputs $\Delta V_{t}$ and $\Delta \omega$ measured 
From the choice of the $Q$ and $R$ matrices for the second observer when the frequency $\Delta \omega$ is measured in addition to the output $\Delta V_{t}$, we can see that the weights of the $R$ matrix are reduced compared to the case when only $\Delta V_{t}$ is measured. Thus, the amount of control effort that can be applied to the system such that all the states and the control inputs remain within a specific bound is more, and hence the state variables and the outputs converge to zero faster compared to the first observer with only $\Delta V_{t}$ measured, but not as fast as the full state feedback LQR controller. This is evident from Figure 7.43 to Figure 7.46 where the estimated states $\Delta \hat{E}_{q}^{\prime}, \Delta \hat{\omega}, \Delta \hat{\delta}, \Delta \hat{T}_{m}, \Delta \hat{G}_{V}$, and the outputs $\Delta V_{t}, \Delta \omega$, and $\Delta \delta$ of the observer based LQR with $\Delta V_{t}$ and $\Delta \omega$ measured take less time to converge to zero as compared to the observer-based LQR with only $\Delta V_{t}$ measured. However, the time taken is larger compared to the LQR with full state feedback, which gives the best results. Figure 7.47 and Figure 7.48 show plots for the two control inputs $\Delta E_{f d}$, and $\Delta u_{T}$ respectively. 


\subsubsection{Observer-Based Pole Placement Controller Design based on linear model}

In this section we design an observer-based pole placement controller for the reduced order linear model of the generator-turbine system connected to an infinite bus. The procedure used to design an observer for the pole placement controller is similar to the one used earlier for the observer-based LQR. The two outputs, generator terminal voltage $\Delta V_{t}$ and frequency $\Delta \omega$, that can be measured using sensors are used for the observer design. Thus we have

$$
\begin{aligned}
\mathbf{y} & =C_{2} \mathbf{x}=\left[\begin{array}{ccccc}
T_{1} & 0 & T_{2} & 0 & 0 \\
0 & 1 & 0 & 0 & 0
\end{array}\right] \mathbf{x} \\
\text { i.e. }\left[\begin{array}{c}
\Delta V_{t} \\
\Delta \omega
\end{array}\right] & =\left[\begin{array}{ccccc}
0.5258 & 0 & 0.0294 & 0 & 0 \\
0 & 1 & 0 & 0 & 0
\end{array}\right] \mathbf{x}
\end{aligned}
$$

The estimator gain matrix $L$ for the output matrix $C_{2}$ as given in Equation (7.32) and $\rho=12$ is

$$
L=\left[\begin{array}{cc}
68.7022 & -2.8308 \\
0.2306 & 18.6252 \\
-608.8626 & 34.4711 \\
-661.5803 & 387.1455 \\
-61.4420 & 58.6700
\end{array}\right]
$$

The desired closed-loop poles of the generator-turbine system for the observer-based pole placement controller were selected as

$$
p=[-0.7,-0.8,-0.5,-0.9,-0.8]
$$

The controller gain matrix $K$ corresponding to these closed loop poles is

$$
K=\left[\begin{array}{ccccc}
9.7459 & -26.9495 & 4.8337 & -0.8674 & -9.0425 \\
0.0299 & -0.2030 & -0.0207 & 0.1337 & -1.1007
\end{array}\right]
$$

From Figure 7.49 to Figure 7.52 we can see that for the observer-based pole placement controller the estimated state variables $\Delta \hat{E}_{q}^{\prime}, \Delta \hat{\omega}, \Delta \hat{\delta}, \Delta \hat{T}_{m}, \Delta \hat{G}_{V}$, and the outputs $\Delta V_{t}, \Delta \omega$, and $\Delta \delta$ converge to zero in approximately 12 seconds. Figure 7.53 and Figure 7.54 show plots for the two control inputs $\Delta E_{f d}$, and $\Delta u_{T}$ respectively. The performance of the observer-based pole placement controller is comparable to the performance of the full-state feedback pole-placement controller. 


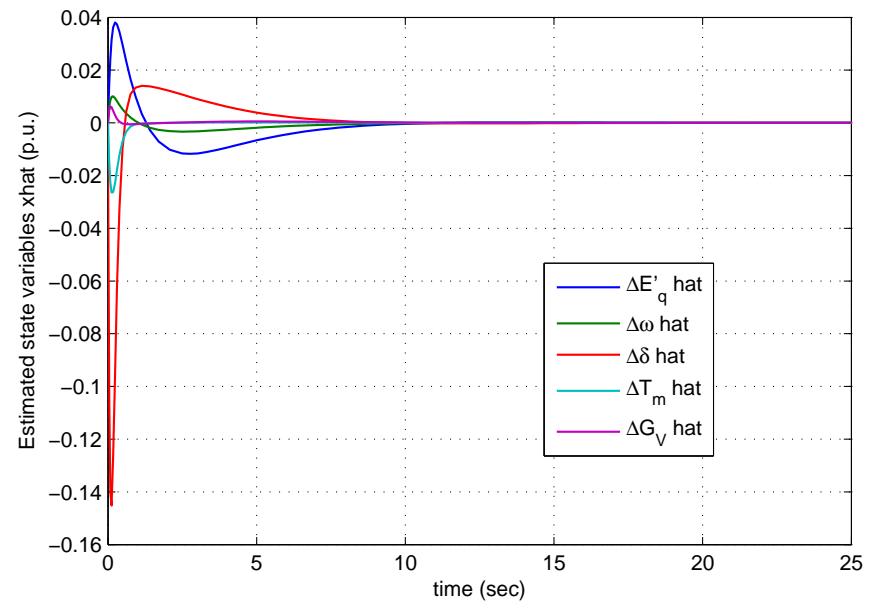

Figure 7.49: Plot of the estimated state variables $\Delta \hat{E}_{q}^{\prime}, \Delta \hat{\omega}, \Delta \hat{\delta}, \Delta \hat{T}_{m}$, and $\Delta \hat{G}_{V}$ vs time for the observerbased pole placement controller applied to the reduced order linear model with outputs $\Delta V_{t}$ and $\Delta \omega$ measured

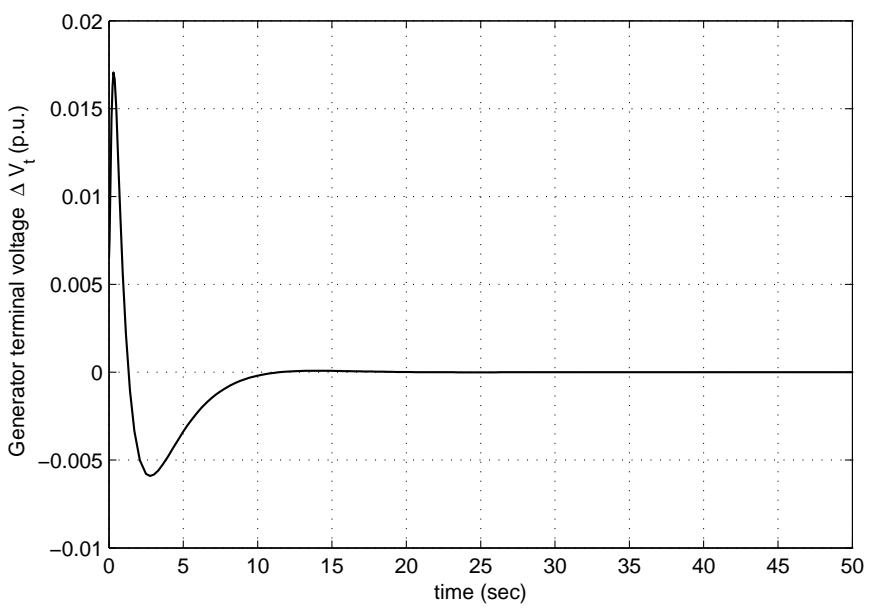

Figure 7.50: Plot of the generator terminal voltage $\Delta V_{t}$ vs time for the observer-based pole placement controller applied to the reduced order linear model with outputs $\Delta V_{t}$ and $\Delta \omega$ measured

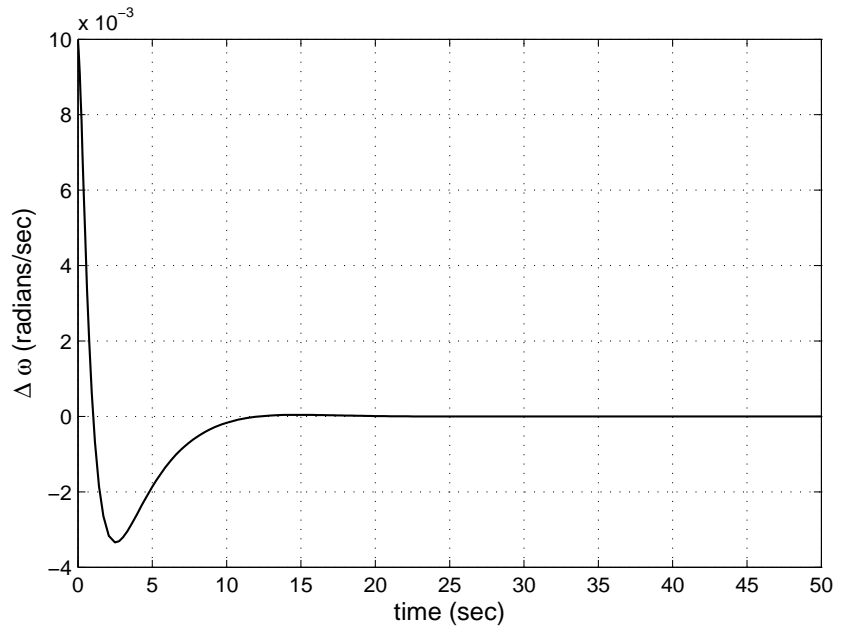

Figure 7.51: Plot of $\Delta \omega$ vs time for the observer-based pole placement controller applied to the reduced order linear model with outputs $\Delta V_{t}$ and $\Delta \omega$ measured 


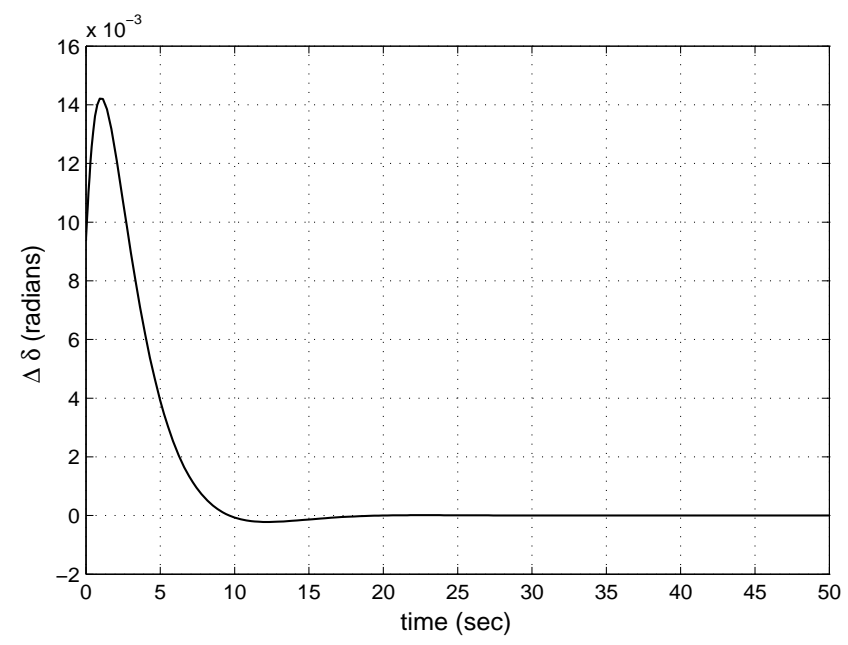

Figure 7.52: Plot of $\Delta \delta$ vs time for the observer-based pole placement controller applied to the reduced order linear model with outputs $\Delta V_{t}$ and $\Delta \omega$ measured

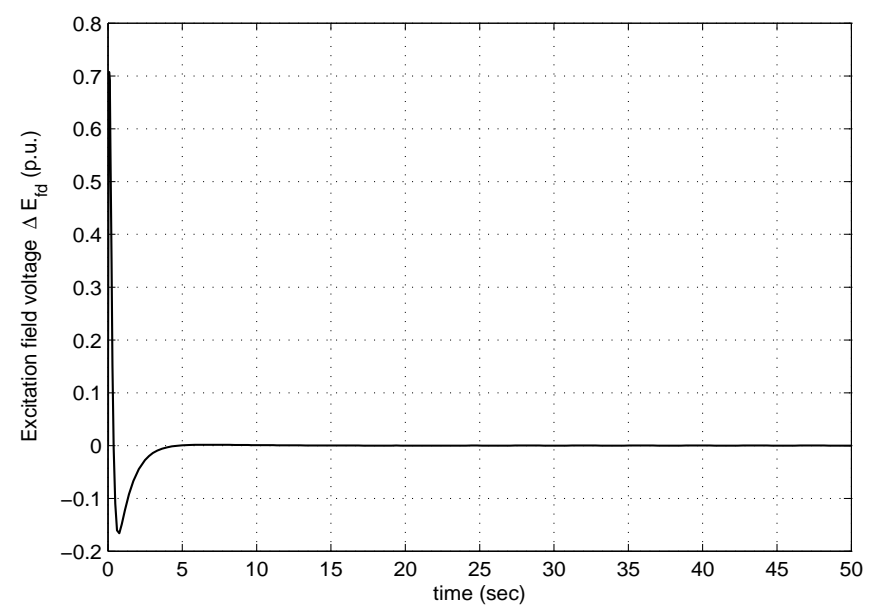

Figure 7.53: Plot of the control input $\Delta E_{f d}$ vs time for the observer-based pole placement controller applied to the reduced order linear model with outputs $\Delta V_{t}$ and $\Delta \omega$ measured

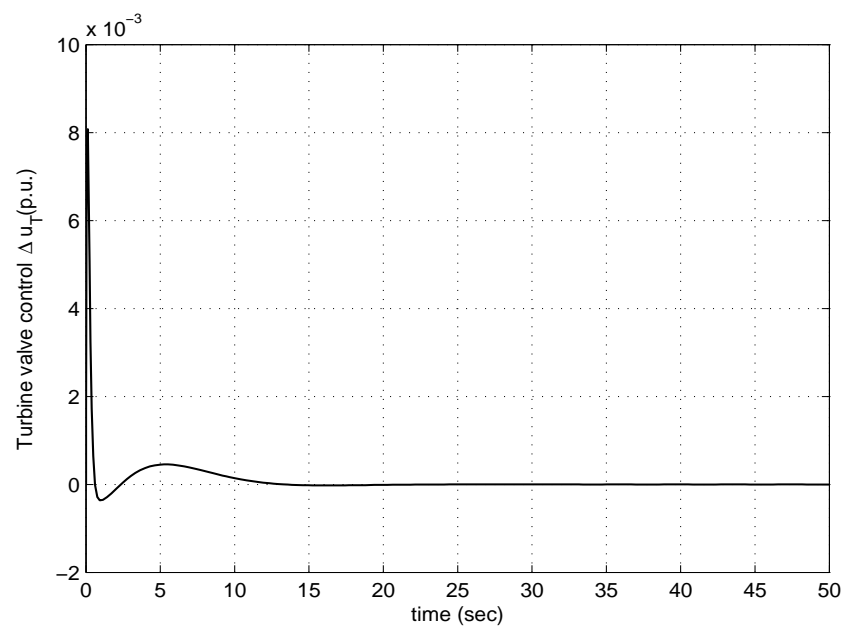

Figure 7.54: Plot of the control input $\Delta u_{T}$ vs time for the observer-based pole placement controller applied to the reduced order linear model with outputs $\Delta V_{t}$ and $\Delta \omega$ measured 


\subsubsection{Simulation Results for the Observer-based LQR applied to the Reduced Order Nonlin- ear Model}

We now test the observer-based LQR on the reduced order nonlinear model. The generator terminal voltage $V_{t}$ and the angular velocity $\omega$ are measured using sensors. The results obtained by directly applying the observer-based LQR that was designed for the reduced order linear model in subsection 7.3.1 are poor. Therefore, the gains of the controller and observer poles are adjusted so that the controller works efficiently on the reduced order nonlinear model. Using the angular velocity or frequency $\omega$ of the synchronous generator which can be measured using a sensor, in addition to the generator terminal voltage $V_{t}$ the output equation can be written as

$$
\begin{aligned}
\mathbf{y} & =C_{2} \mathbf{x}=\left[\begin{array}{ccccc}
T_{1} & 0 & T_{2} & 0 & 0 \\
0 & 1 & 0 & 0 & 0
\end{array}\right] \mathbf{x} \\
\text { i.e. }\left[\begin{array}{c}
\Delta V_{t} \\
\Delta \omega
\end{array}\right] & =\left[\begin{array}{ccccc}
0.5258 & 0 & 0.0294 & 0 & 0 \\
0 & 1 & 0 & 0 & 0
\end{array}\right] \mathbf{x}
\end{aligned}
$$

The estimator gain matrix $L$ for the output matrix $C_{2}$ as given in Equation (7.41) and $\rho=12$ is

$$
L=\left[\begin{array}{cc}
145.5739 & 29.0262 \\
-9.1126 & 22.2527 \\
-528.9546 & -442.3042 \\
-2676.907 & -678.8487 \\
834.9962 & 219.7840
\end{array}\right]
$$

Choosing the weighting matrices

$$
Q=\left[\begin{array}{ccccc}
5 & 0 & 0 & 0 & 0 \\
0 & 5 & 0 & 0 & 0 \\
0 & 0 & 0.5 & 0 & 0 \\
0 & 0 & 0 & 0.05 & 0 \\
0 & 0 & 0 & 0 & 5
\end{array}\right]
$$

and

$$
R=\left[\begin{array}{cc}
1000 & 0 \\
0 & 1000
\end{array}\right]
$$

and the state space matrices $(A, B)$ as given in Equation (3.76) and Equation (3.77) the control gain $K$ for the observer-based LQR with the outputs $V_{t}$ and $\omega$ measured is found to be

$$
K=\left[\begin{array}{ccccc}
0.0021 & -0.0027 & 0.0005 & -0.0003 & -0.0001 \\
-0.0036 & 0.0079 & -0.0014 & 0.0009 & 0.0028
\end{array}\right]
$$

Figure 7.55, Figure 7.56, and Figure 7.57 show simulation results for the re-tuned observer-based LQR applied to the reduced order nonlinear model. From these simulation results we can see that the generator terminal voltage $V_{t}$ oscillates between 0.8 and 0.82 p.u. which is different from the desired steady state value of 1.172 p.u. by an amount of 0.372 p.u., the rotor angle $\delta$ oscillates between 0 and 0.4 p.u. with the amplitude of oscillations decreasing with time, which is also different from the desired steady state value of 1 p.u., and the frequency $\omega$ oscillates about the desired steady state value of 1 p.u. Even after re-tuning the gains of the controller and the observer poles it is not possible to improve the results too much. A significant amount of steady state error is observed. The linear observer is not able to accurately estimate the states of the reduced order nonlinear model, i.e. it is not robust to uncertainties or state deviations between the reduced order nonlinear and the reduced order linear model. To overcome this difficulty i.e. to increase the robustness of the observer we design a linear quadratic gaussian (LQG) controller, in which the gains of the Kalman filter are tuned using loop transfer recover (LTR) procedure, in the next subsection. 


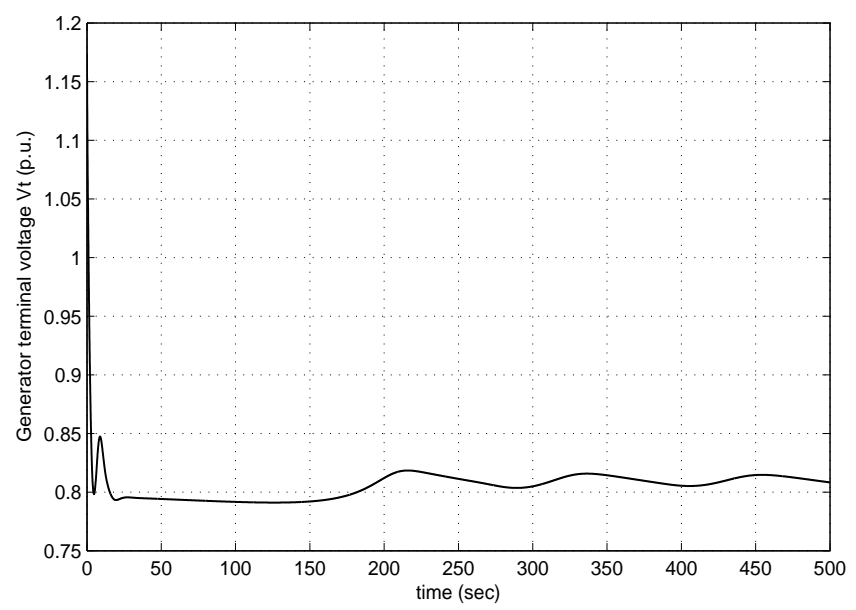

Figure 7.55: Plot of the generator terminal voltage $V_{t}$ vs time for the observer-based LQR applied to the reduced order nonlinear model

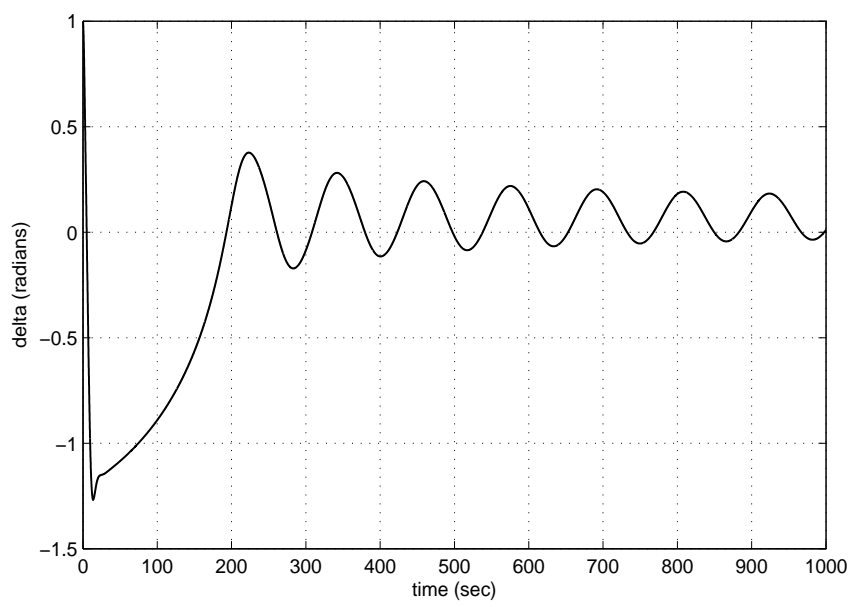

Figure 7.56: Plot of the rotor angle $\delta$ vs time for the observer-based LQR applied to the reduced order nonlinear model

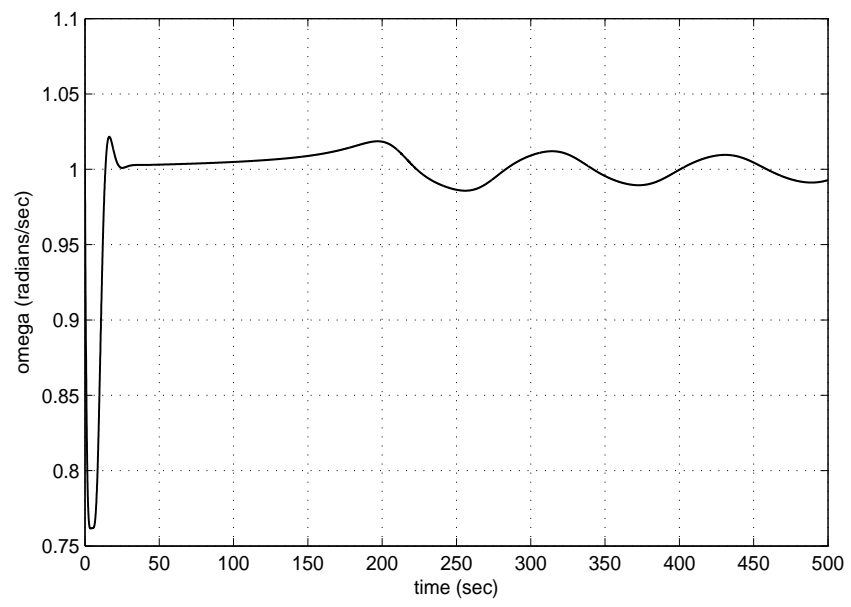

Figure 7.57: Plot of the frequency $\omega$ vs time for the observer-based LQR applied to the reduced order nonlinear model 


\subsubsection{LTR-based LQG Controller applied to the Reduced Order Nonlinear Model}

In this section we present the design of a linear quadratic Gaussian (LQG) controller, where a design or gain adjustment procedure in the time domain is used which is analogous to loop shaping in the frequency domain, to adjust the gains of the Kalman filter. This gain adjustment procedure not only improves the robustness of the observer but also asymptotically achieves the same loop transfer function as a full-state feedback controller [3]. A basic requirement for every point of an adjustment trajectory is stability of the observer error dynamics. If this requirement is not satisfied then closed-loop stability of the system is also lost. One way to assure stable error dynamics is to restrict the observer to be a Kalman filter for some set of noise parameters or covariance matrices $V_{1}$ and $V_{2}$, where $V_{1}$ is a $5 \times 5$ matrix of intensities related to the plant disturbances or process noise, and $V_{2}$ is a $2 \times 2$ matrix of intensities related to the measurement or sensor noise. Thus let the observer gain $H(q)$ be given by the Kalman filter expression

$$
H(q)=\Sigma(q) C^{T} V_{2}^{-1}
$$

with $\Sigma(q)$ defined by the Riccati equation

$$
A \Sigma(q)+\Sigma(q) A^{T}+V_{1}(q)-\Sigma(q) C^{T} V_{2}^{-1} C \Sigma(q)=0
$$

We select $V_{1}=V_{1}^{T}>0$ and $V_{2}=V_{2}^{T}>0$ with $\left(A, V_{1}^{\frac{1}{2}}\right)$ and $(C, A)$ stabilizable and observable, respectively. $V_{1}(q)$ which is a function of $q$ is designed as

$$
\begin{aligned}
& V_{1}(q)=V_{10}+q^{2} B V B^{T} \\
& V_{2}(q)=V_{20}
\end{aligned}
$$

where $V_{10}$ and $V_{20}$ are noise intensities appropriate for the nominal plant, and $V$ is any positive definite symmetric matrix, which are chosen as

$$
\begin{aligned}
V_{10} & =\left[\begin{array}{lllll}
1 & 0 & 0 & 0 & 0 \\
0 & 1 & 0 & 0 & 0 \\
0 & 0 & 1 & 0 & 0 \\
0 & 0 & 0 & 1 & 0 \\
0 & 0 & 0 & 0 & 1
\end{array}\right] \\
V_{20} & =\left[\begin{array}{ll}
1 & 0 \\
0 & 1
\end{array}\right] \\
V & =\left[\begin{array}{ll}
1 & 0 \\
0 & 1
\end{array}\right]
\end{aligned}
$$

With these selections, the observer gain for $q=0$ corresponds to the nominal Kalman filter gain. However as $q$ approaches infinity, the gains are seen from Equation (7.47) to satisfy

$$
\frac{H V_{2} H^{T}}{q^{2}} \rightarrow B V B^{T}
$$

By substituting Equation (7.48) in Equation (7.47) and dividing Equation (7.47) by $q^{2}$ we get

$$
A\left(\frac{\Sigma(q)}{q^{2}}\right)+\left(\frac{\Sigma(q)}{q^{2}}\right) A^{T}+\frac{V_{10}}{q^{2}}+B V B^{T}-q^{2}\left(\frac{\Sigma(q)}{q^{2}}\right) C^{T} V_{2}^{-1} C\left(\frac{\Sigma(q)}{q^{2}}\right)=0
$$

From [3] it can be seen that

$$
\frac{\Sigma(q)}{q^{2}} \rightarrow 0 \quad \text { as } q \rightarrow \infty
$$

whenever the transfer function $C(s I-A)^{-1} B$ has no right half plane zeros. Consequently,

$$
q^{2}\left(\frac{\Sigma(q)}{q^{2}}\right) C^{T} V_{2}^{-1} C\left(\frac{\Sigma(q)}{q^{2}}\right) \rightarrow B V B^{T}
$$


and thus Equation (7.50) is established. Solutions of Equation (7.50) must necessarily be of the form

$$
\frac{H(q)}{q} \rightarrow B V^{\frac{1}{2}}\left(V_{2}^{\frac{1}{2}}\right)^{-1}
$$

where $V^{\frac{1}{2}}$ denotes some square root of $V$, i.e. $\left(V^{\frac{1}{2}}\right)^{T} V^{\frac{1}{2}}=V$, and similarly $V_{2}^{\frac{1}{2}}$ is some square root of $V_{2}$. The design adjustment procedure defined by Equation (7.46) to Equation (7.48) will achieve the desired robustness improvement objective. We can see that higher the value of $q$, higher will be the observer gain $H$ and thus higher will be the robustness of the system which is maximum at $q=\infty$. Our objective is to apply the LTR-based LQG controller on the reduced order nonlinear model. From simulations it is verified that for very large values of $q$ the system goes unstable. Thus the choice of $q$ is very important and it cannot be arbitrarily selected to a large value, when we are applying this design adjustment procedure on a nonlinear model. The design parameter $q$ is suitable selected to be equal to 9.0005 to get satisfactory closed loop performance of the system. From the separation principle we know that the gains of the Kalman filter $H(q)$ and the controller gains $K$ can be deigned independently. We use the LQR algorithm to design the controller gains. Thus by using the feedback law,

$$
\mathbf{u}=-K \hat{\mathbf{x}}
$$

where $\hat{\mathbf{x}}$ are the states estimated by the Kalman filter with loop shaping, the weighting matrices

$$
Q=\left[\begin{array}{ccccc}
1254.75 & 0 & 0 & 0 & 0 \\
0 & 1500 & 0 & 0 & 0 \\
0 & 0 & 544.5 & 0 & 0 \\
0 & 0 & 0 & 142.5 & 0 \\
0 & 0 & 0 & 0 & 1500
\end{array}\right]
$$

and

$$
R=\left[\begin{array}{ll}
1 & 0 \\
0 & 1
\end{array}\right]
$$

and the state space matrices $(A, B)$ as given in Equation (3.82) and Equation (3.83) the control gain $K$ is found to be

$$
K=\left[\begin{array}{ccccc}
34.5065 & -49.5197 & -5.6995 & -4.1955 & -0.0432 \\
-1.2745 & 28.0922 & -0.0281 & 4.3245 & 37.7873
\end{array}\right]
$$

Figure 7.58 to Figure 7.63 show simulation results for the LTR-based LQG controller applied to the reduced order nonlinear model. From these results we can see that all the state variables, outputs, and control inputs, attain their desired steady state values respectively. Thus a significant improvement in the robustness is observed when the Kalman filter gains are tuned using the loop-shaping design adjustment procedure explained above. The performance of the LTR-based LQG controller is comparable to that of the LQRbased full-state feedback controller. 


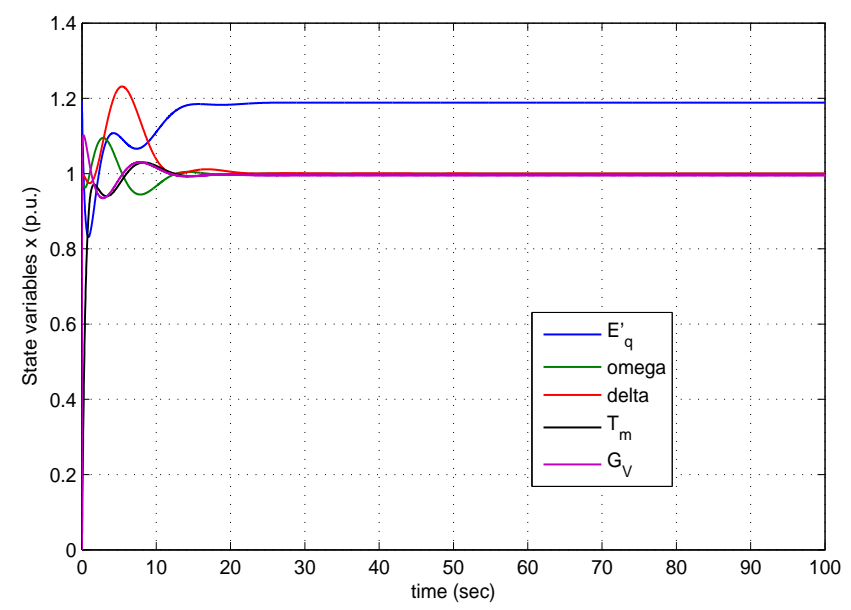

Figure 7.58: Plot of the state variables $E_{q}^{\prime}, \omega, \delta, T_{m}$, and $G_{V}$ vs time for the LTR-based LQG controller applied to the reduced order nonlinear model

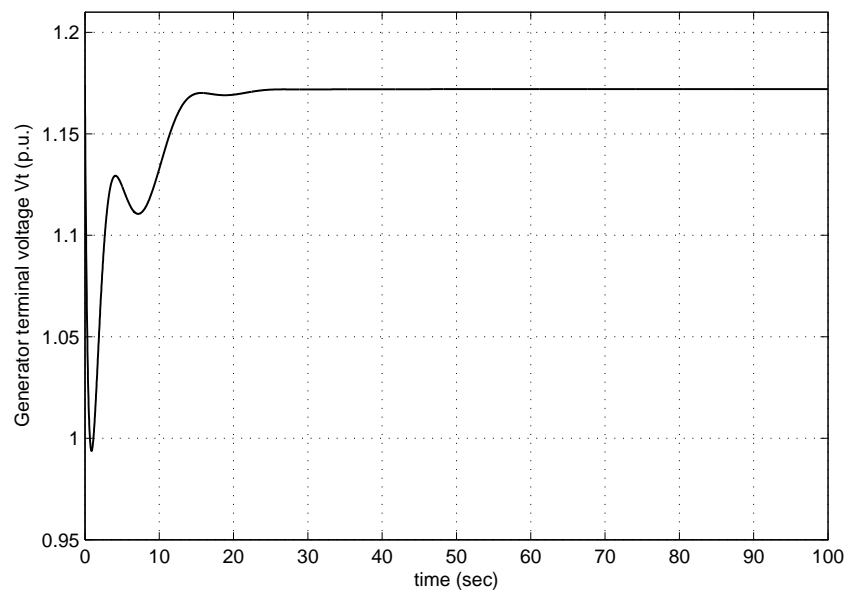

Figure 7.59: Plot of the generator terminal voltage $V_{t}$ vs time for the LTR-based LQG controller applied to the reduced order nonlinear model

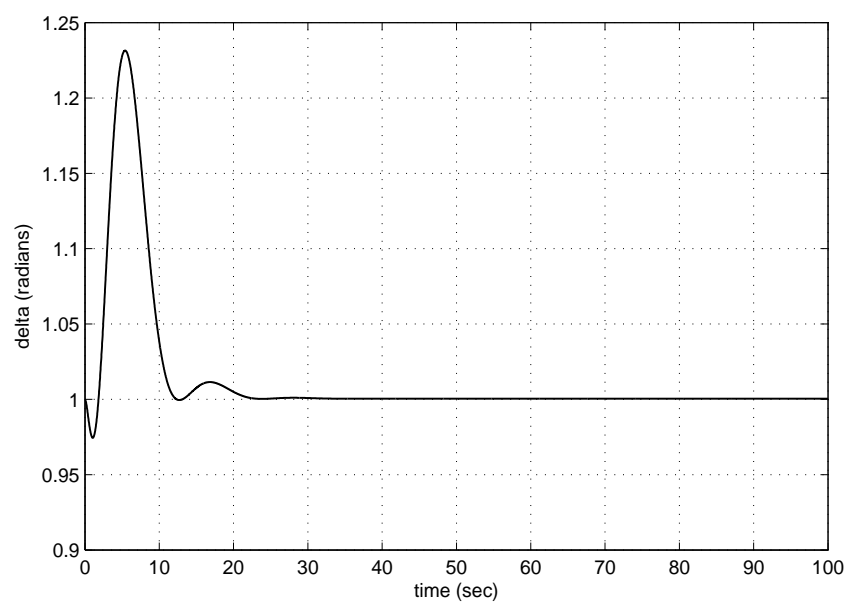

Figure 7.60: Plot of the rotor angle $\delta$ vs time for the LTR-based LQG controller applied to the reduced order nonlinear model 


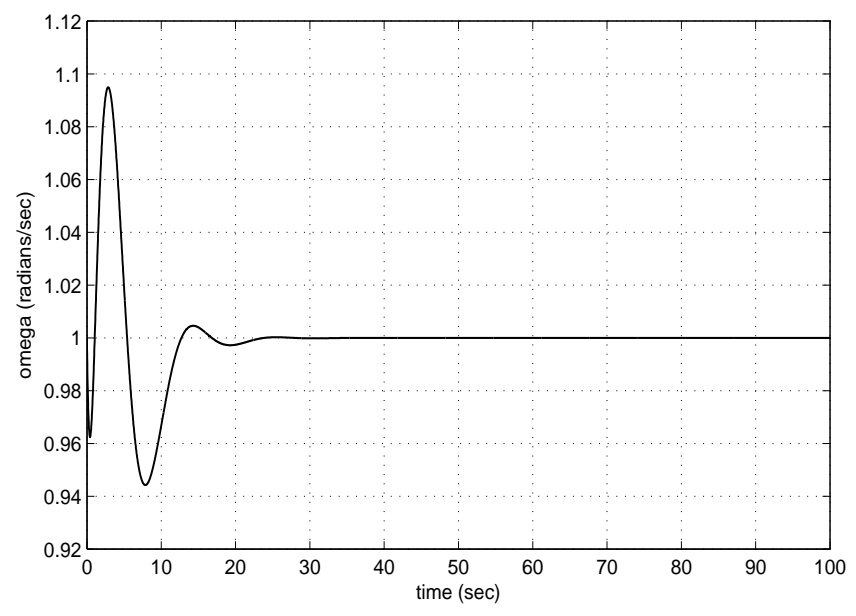

Figure 7.61: Plot of the frequency $\omega$ vs time for the LTR-based LQG controller applied to the reduced order nonlinear model

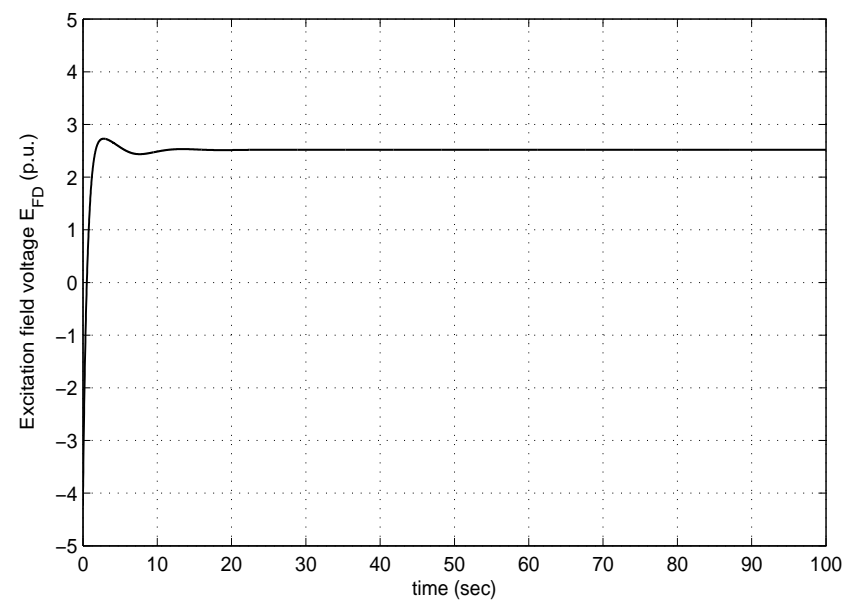

Figure 7.62: Plot of the generator excitation field control $E_{F D}$ vs time for the LTR-based LQG controller applied to the reduced order nonlinear model

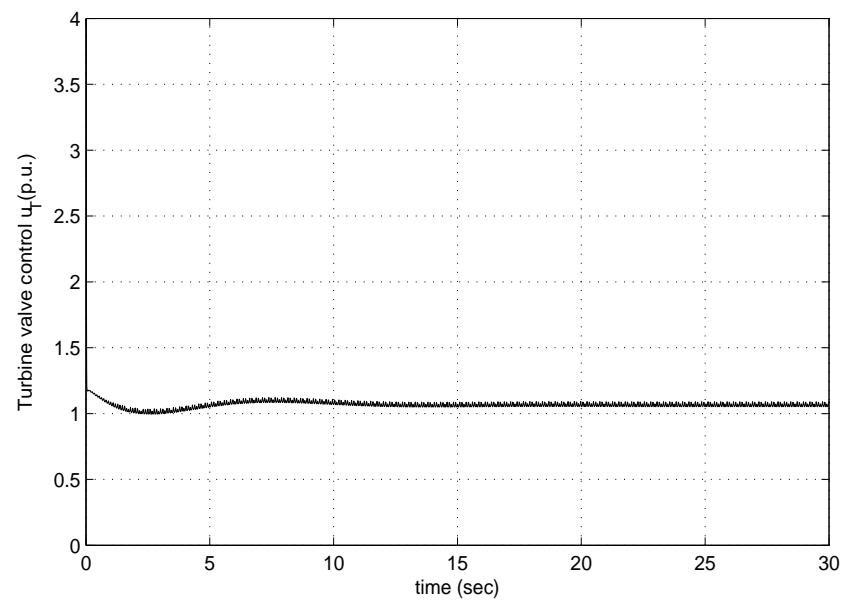

Figure 7.63: Plot of the turbine valve control $u_{T}$ vs time for the LTR-based LQG controller applied to the reduced order nonlinear model 


\subsubsection{LTR-based LQG Controller applied to the Truth Model}

It is not always possible to measure the rotor angle $\delta$ of the synchronous generator. Thus in cases where the rotor angle cannot be measured, state feedback based control techniques are not applicable to the truth model. Hence it is necessary to design an observer-based output feedback controller which can be applied to the truth model.

The loop transfer recovery (LTR)-based linear quadratic gaussian (LQG) controller that was tested on the reduced order nonlinear model, is now tested on the truth model. The covariance matrices $V_{1}, V_{2}$, that are used in the design of the Kalman filter, the loop shaping parameter $q$, and the controller gains are tuned once again so that the LTR-based LQG controller works efficiently on the truth model. We select $V_{1}=V_{1}^{T}>0$ and $V_{2}=V_{2}^{T}>0$ with $\left(A, V_{1}^{\frac{1}{2}}\right)$ and $(C, A)$ stabilizable and observable, respectively. $V_{1}(q)$ which is a function of $q$ is designed as

$$
\begin{aligned}
& V_{1}(q)=V_{10}+q^{2} B V B^{T} \\
& V_{2}(q)=V_{20}
\end{aligned}
$$

$V_{10}$ and $V_{20}$ are noise intensities appropriate for the nominal plant, and $V$ is any positive definite symmetric matrix, which are chosen as

$$
\begin{aligned}
V_{10} & =\left[\begin{array}{lllll}
1 & 0 & 0 & 0 & 0 \\
0 & 1 & 0 & 0 & 0 \\
0 & 0 & 1 & 0 & 0 \\
0 & 0 & 0 & 1 & 0 \\
0 & 0 & 0 & 0 & 1
\end{array}\right] \\
V_{20} & =\left[\begin{array}{cc}
0.65 & 0 \\
0 & 0.65
\end{array}\right] \\
V & =\left[\begin{array}{ll}
1 & 0 \\
0 & 1
\end{array}\right]
\end{aligned}
$$

The loop shaping parameter $q$ is tuned to 5.25. We use the LQR algorithm to design the controller gains.

Thus by using the feedback law,

$$
\mathbf{u}=-K \hat{\mathbf{x}}
$$

where $\hat{\mathbf{x}}$ are the states estimated by the Kalman filter with loop shaping, the weighting matrices

$$
Q=\left[\begin{array}{ccccc}
7500 & 0 & 0 & 0 & 0 \\
0 & 15000 & 0 & 0 & 0 \\
0 & 0 & 16500 & 0 & 0 \\
0 & 0 & 0 & 7500 & 0 \\
0 & 0 & 0 & 0 & 7500
\end{array}\right]
$$

and

$$
R=\left[\begin{array}{ll}
1 & 0 \\
0 & 1
\end{array}\right]
$$

and the state space matrices $(A, B)$ as given in Equation (3.82) and Equation (3.83) the control gain $K$ is found to be

$$
K=\left[\begin{array}{ccccc}
87.3944 & -216.7677 & -60.7947 & -13.4353 & -0.0618 \\
-1.8244 & 98.0650 & 17.7303 & 42.1399 & 85.8027
\end{array}\right]
$$

Figure 7.64 to Figure 7.68 show simulation results for the LTR-based LQG controller applied to the truth model. From Figure 7.64, Figure 7.65, and Figure 7.66 we can see that $V_{t}, \omega$, and $\delta$ settle to their respective steady state values. Small oscillations which decay with time are seen for $\omega$, and $\delta$. Figure 7.67 and Figure 7.68 show the plots for the two control inputs $V_{F}$ and $u_{T}$ respectively. The generator excitation voltage $V_{F}$ settles to its steady state value of 0.00121 p.u. and the turbine valve control settles to its steady state value of 1.0512 p.u. 


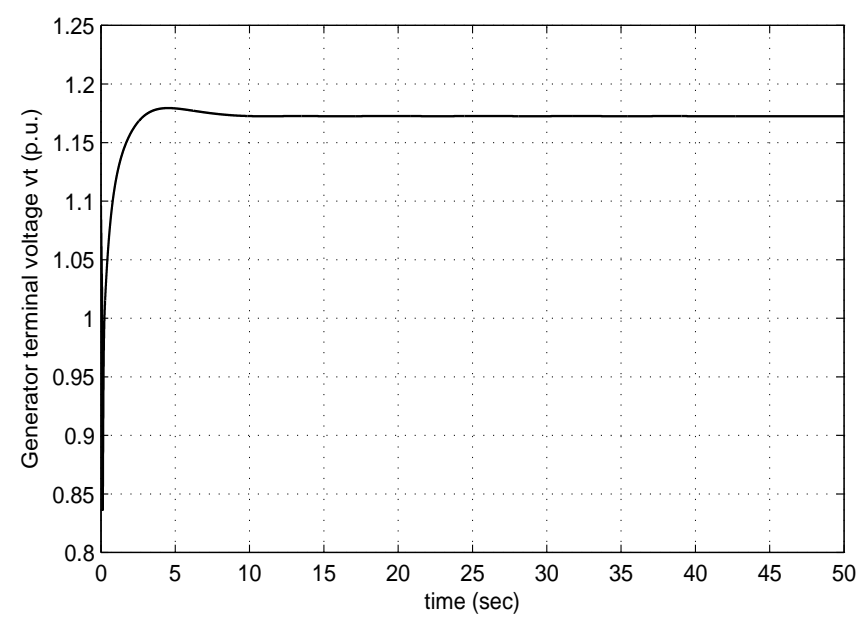

Figure 7.64: Plot of the generator terminal voltage $V_{t}$ vs time for the LTR-based LQG controller applied to the truth model

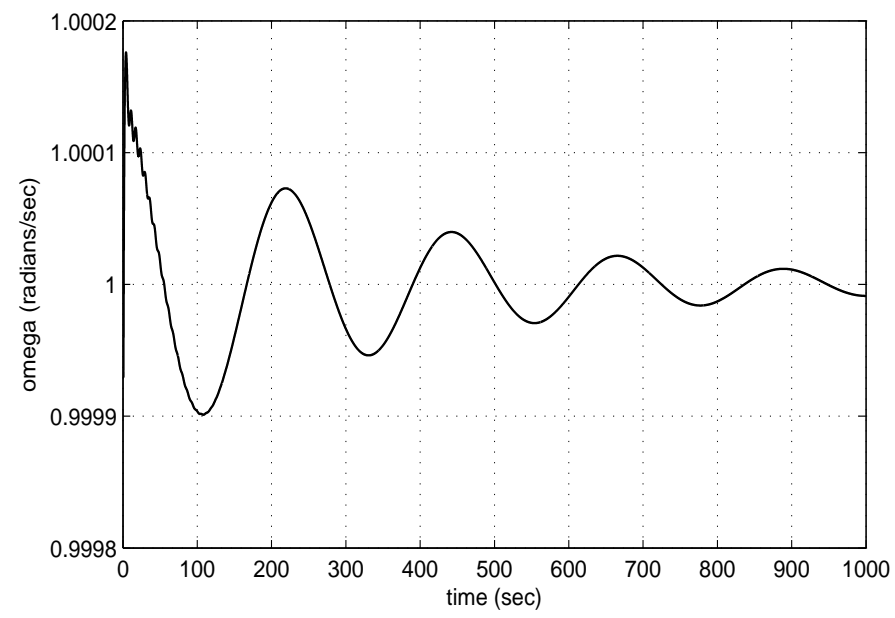

Figure 7.65: Plot of the angular velocity $\omega$ vs time for the LTR-based LQG controller applied to the truth model

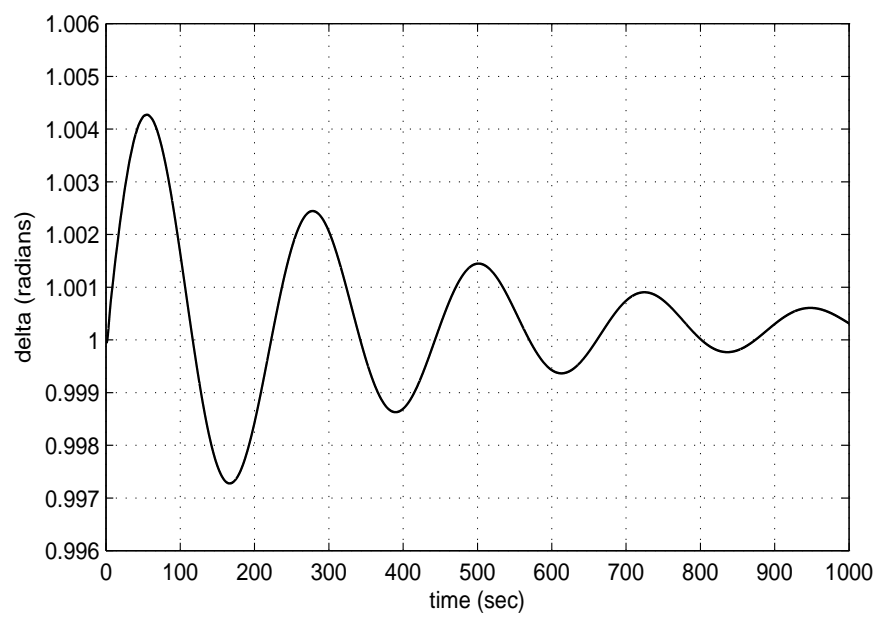

Figure 7.66: Plot of the rotor angle $\delta$ vs time for the LTR-based LQG controller applied to the truth model 


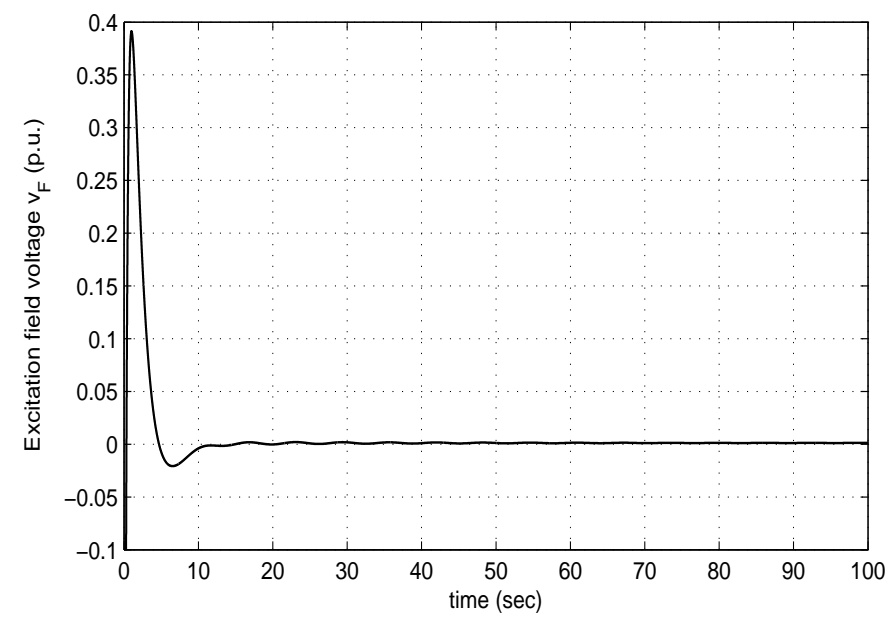

Figure 7.67: Plot of the generator excitation voltage $V_{F}$ vs time for the LTR-based LQG controller applied to the truth model

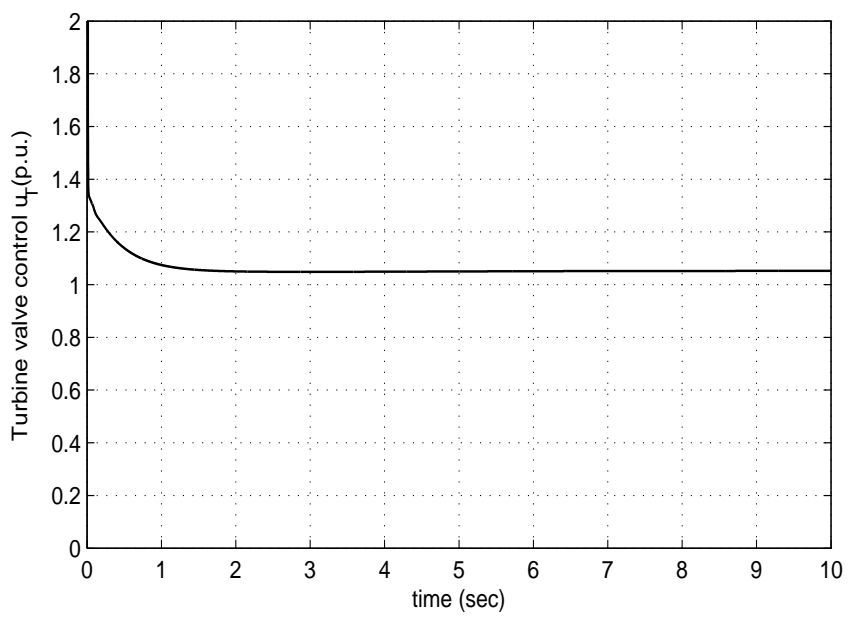

Figure 7.68: Plot of the turbine valve control $u_{T}$ vs time for the LTR-based LQG controller applied to the truth model 


\section{Nonlinear Feedback Linearizing Controller Design}

We present the design of a nonlinear input-state feedback linearizing controller for the nonlinear model of the synchronous generator and turbine connected to an infinite bus. Before we proceed to the nonlinear controller design, we give the fundamentals of full-state feedback linearization for single-input, single-output (SISO) nonlinear control systems [5,6], which are affine in the control input, that is, systems of the form

$$
\begin{aligned}
& \dot{x}=f(x)+g(x) u \\
& y=h(x)
\end{aligned}
$$

where $x \in \mathbb{R}^{n}, u \in \mathbb{R}, y \in \mathbb{R}$. We assume that the vector fields $f: D \rightarrow \mathbb{R}^{n}, g: D \rightarrow \mathbb{R}^{n}$ and the readout map $h: D \rightarrow \mathbb{R}$ are smooth in the domain $D \subset \mathbb{R}^{n}$, that is their partial derivatives with respect to $x$ of any order exist and are continuous in $D$. Our objective is to find a smooth full state feedback control law of the form

$$
u=\alpha(x)+\beta(x) v
$$

and a change of variables $z=T(x)$ that transform the nonlinear system into an equivalent linear system, i.e. the closed loop system

$$
\begin{aligned}
& \dot{x}=f(x)+g(x) \alpha(x)+g(x) \beta(x) v \\
& y=h(x)
\end{aligned}
$$

is completely linearized. The ability to use state feedback control to convert a nonlinear state equation into a controllable linear state equation by canceling nonlinearities requires the nonlinear state equation to have the structure

$$
\dot{x}=A x+B \gamma(x)[u-\alpha(x)]
$$

where $A$ is $n \times n, B$ is $n \times p$, the pair $(A, B)$ is controllable, the functions $\alpha: \mathbb{R}^{n} \rightarrow \mathbb{R}^{p}$ and $\gamma: \mathbb{R}^{n} \rightarrow \mathbb{R}^{p \times p}$ are defined in a domain $D \subset \mathbb{R}^{n}$ that contains the origin, and the matrix $\gamma(x)$ is nonsingular for every $x \in D$. If the state equation takes the form Equation (8.4), then we can linearize it via the state feedback

$$
u=\alpha(x)+\beta(x) v
$$

where $\beta(x)=\gamma^{-1}(x)$, to obtain the linear state equation

$$
\dot{x}=A x+B v
$$

For stabilization, we design $v=-K x$ such that $A-B K$ is Hurwitz. The overall nonlinear stabilizing state feedback control is

$$
u=\alpha(x)-\beta(x) K x
$$

\section{Example $1[6]$}

To introduce the idea of exact feedback linearization, let us consider the pendulum stabilization problem. Inspection of the state equation

$$
\begin{aligned}
& \dot{x}_{1}=x_{2} \\
& \dot{x}_{2}=-a\left[\sin \left(x_{1}+\delta\right)-\sin \delta\right]-b x_{2}+c u
\end{aligned}
$$

shows that we can choose $u$ as

$$
u=\left(\frac{a}{c}\right)\left[\sin \left(x_{1}+\delta\right)-\sin \delta\right]+\frac{v}{c}
$$

to cancel the nonlinear term $a\left[\sin \left(x_{1}+\delta\right)-\sin \delta\right]$. This cancellation results in the linear system

$$
\begin{aligned}
& \dot{x}_{1}=x_{2} \\
& \dot{x}_{2}=-b x_{2}+v
\end{aligned}
$$


Thus, the stabilization problem for the nonlinear system has been reduced to a stabilization problem for a controllable linear system. We can proceed to design a stabilizing linear state feedback control

$$
v=k_{1} x_{1}+k_{2} x_{2}
$$

to locate the eigenvalues of the closed-loop system

$$
\begin{aligned}
& \dot{x}_{1}=x_{2} \\
& \dot{x}_{2}=k_{1} x_{1}+\left(k_{2}-b\right) x_{2}
\end{aligned}
$$

in the open left-half plane. The overall state feedback control law is given by

$$
u=\left(\frac{a}{c}\right)\left[\sin \left(x_{1}+\delta\right)-\sin \delta\right]+\frac{1}{c}\left(k_{1} x_{1}+k_{2} x_{2}\right)
$$

However, if the nonlinear state equation does not have the structure of Equation (8.4) for one choice of state variables, it does not mean that we cannot linearize the system via feedback. A co-ordinate transformation which transforms the old states into new state variables, can be used to convert the nonlinear state equation in the original states into a structure of Equation (8.4) in the new state variables.

\section{Example $2[6]$}

Consider the system

$$
\begin{aligned}
& \dot{x}_{1}=a \sin x_{2} \\
& \dot{x}_{2}=-x_{1}^{2}+u
\end{aligned}
$$

We cannot simply choose $u$ to cancel the nonlinear term $a \sin x_{2}$. However, if we first change the variables by the transformation

$$
\begin{aligned}
& z_{1}=x_{1} \\
& z_{2}=a \sin x_{2}=\dot{x}_{1}
\end{aligned}
$$

then $z_{1}$ and $z_{2}$ satisfy

$$
\begin{aligned}
& \dot{z}_{1}=z_{2} \\
& \dot{z}_{2}=a \cos x_{2}\left(-x_{1}^{2}+u\right)
\end{aligned}
$$

and the nonlinearities can be canceled by the control

$$
u=x_{1}^{2}+\frac{1}{a \cos x_{2}} v
$$

which is well defined for $-\frac{\pi}{2}<x_{2}<\frac{\pi}{2}$. The state equation in the new coordinates $\left(z_{1}, z_{2}\right)$ can be found by inverting the transformation to express $\left(x_{1}, x_{2}\right)$ in terms of $\left(z_{1}, z_{2}\right)$; that is,

$$
\begin{aligned}
& x_{1}=z_{1} \\
& x_{2}=\sin ^{-1}\left(\frac{z_{2}}{a}\right)
\end{aligned}
$$

which is well defined for $-a<z_{2}<a$. The transformed state equation is given by

$$
\begin{aligned}
& \dot{z}_{1}=z_{2} \\
& \dot{z}_{2}=a \cos \left(\sin ^{-1}\left(\frac{z_{2}}{a}\right)\right)\left(-z_{1}^{2}+u\right)
\end{aligned}
$$

When a change of variables $z=T(x)$ is used to transform the state equation from the $x$-coordinates to the $z$-coordinates, the map $T$ must be invertible; that is it must have an inverse map $T^{-1}(\cdot)$ such that $x=T^{-1}(z)$ for all $z \in T(D)$, where $D$ is the domain of $T$. Moreover, because the derivatives of $z$ and $x$ 
should be continuous, we require both $T(\cdot)$ and $T^{-1}(\cdot)$ to be continuously differentiable. A continuously differentiable map with a continuously differentiable inverse is known as diffeomorphism [5, 6]. Thus, we can define a feedback linearizable system as

Definition [6]: A nonlinear system

$$
\begin{aligned}
& \dot{x}=f(x)+g(x) u \\
& y=h(x)
\end{aligned}
$$

where $f: D \rightarrow \mathbb{R}^{n}$ and $g: D \rightarrow \mathbb{R}^{n \times p}$ are sufficiently smooth on a domain $D \subset \mathbb{R}^{n}$, is said to be feedback linearizable (or input-state linearizable) if there exists a diffeomorphism $T: D \rightarrow \mathbb{R}^{n}$ such that $D_{z}=T(D)$ contains the origin and the change of variables $z=T(x)$ transforms the system into the form

$$
\dot{z}=A z+B \gamma(x)[u-\alpha(x)]
$$

with $(A, B)$ controllable and $\gamma(x)$ nonsingular for all $x \in D$

\subsection{Nonlinear Feedback Linearizing Controller Design for the Reduced Order Model}

We now present the design of an input-state feedback linearizing controller for the reduced order model of the synchronous generator and turbine-governor system connected to an infinite bus. The fifth order nonlinear model of the system in the original coordinates is

$$
\begin{aligned}
& \dot{E}_{q}^{\prime}=f_{11} E_{q}^{\prime}+f_{12} \cos (\delta-\alpha)+f_{13} \sin (\delta-\alpha)+g_{11} E_{F D} \\
& \dot{\omega}=f_{21} E_{q}^{\prime 2}+f_{22} E_{q}^{\prime} \cos (\delta-\alpha)+f_{23} E_{q}^{\prime} \sin (\delta-\alpha)+f_{24} \sin (\delta-\alpha) \cos (\delta-\alpha) \\
& \quad+f_{25} \cos ^{2}(\delta-\alpha)+f_{26} \sin ^{2}(\delta-\alpha)+f_{27} \omega+f_{28} T_{m} \\
& \dot{\delta}=\omega-1 \\
& \dot{T}_{m}=f_{41} T_{m}+f_{42} G_{V} \\
& \dot{G}_{V}=f_{51} \omega+f_{52} G_{V}+g_{55} u_{T}
\end{aligned}
$$

where $x=\left[E_{q}^{\prime}, \omega, \delta, T_{m}, G_{V}\right]^{\mathrm{T}}$ are the original state variables and $u=\left[u_{1}, u_{2}\right]^{\mathrm{T}}=\left[E_{F D}, u_{T}\right]^{\mathrm{T}}$ are the two control inputs. We now define a transformation $z=T(x)$ that transforms the nonlinear state equations into a structure of Equation (8.4). The new state variables are chosen as

$$
\begin{aligned}
& z_{1}=\delta \\
& z_{2}=\dot{z}_{1}=\dot{\delta}=\omega-1 \\
& z_{3}=\dot{z}_{2}=\ddot{\delta}=\dot{\omega}=f_{21} E_{q}^{\prime 2}+f_{22} E_{q}^{\prime} \cos (\delta-\alpha)+f_{23} E_{q}^{\prime} \sin (\delta-\alpha)+f_{24} \sin (\delta-\alpha) \cos (\delta-\alpha) \\
& +f_{25} \cos ^{2}(\delta-\alpha)+f_{26} \sin ^{2}(\delta-\alpha)+f_{27} \omega+f_{28} T_{m} \\
& z_{4}=T_{m} \\
& z_{5}=\dot{z}_{4}=\dot{T}_{m}=f_{41} T_{m}+f_{42} G_{V}
\end{aligned}
$$

Thus, we have

$$
\begin{aligned}
& \dot{z}_{1}=\dot{\delta}=\omega-1=z_{2} \\
& \dot{z}_{2}=\dot{\omega}=\ddot{\delta}=z_{3} \\
& \dot{z}_{3}=\ddot{\omega}=\dddot{\delta}=\sigma_{1}(x)+\gamma_{1}(x) E_{F D} \\
& \dot{z}_{4}=\dot{T}_{m}=z_{5} \\
& \dot{z}_{5}=\ddot{T}_{m}=\sigma_{2}(x)+\gamma_{2}(x) u_{T}
\end{aligned}
$$


The state transformations given above are invertible and exist throughout the domain of stable operation $0<\delta<180^{\circ}$. From Equation (8.22) and Equation (8.24), i.e. by taking the derivative of $\dot{\omega}$ we have

$$
\begin{aligned}
\dot{z}_{3}= & \ddot{\omega}=2 f_{21} E_{q}^{\prime} \dot{E}_{q}^{\prime}+f_{22} \dot{E}_{q}^{\prime} \cos (\delta-\alpha)-f_{22} E_{q}^{\prime} \sin (\delta-\alpha) \dot{\delta}+f_{23} \dot{E}_{q}^{\prime} \sin (\delta-\alpha) \\
& +f_{23} E_{q}^{\prime} \cos (\delta-\alpha) \dot{\delta}+f_{24} \cos ^{2}(\delta-\alpha) \dot{\delta}-f_{24} \sin ^{2}(\delta-\alpha) \dot{\delta}-2 f_{25} \cos (\delta-\alpha) \sin (\delta-\alpha) \dot{\delta} \\
& +2 f_{26} \cos (\delta-\alpha) \sin (\delta-\alpha) \dot{\delta}+f_{27} \dot{\omega}+f_{28} \dot{T}_{m}
\end{aligned}
$$

Substituting $\dot{\delta}, \dot{E}_{q}^{\prime}, \dot{\omega}$, and $\dot{T}_{m}$ from Equation (8.22) in Equation (8.25) we get

$$
\begin{aligned}
\dot{z}_{3}= & 2 f_{21} E_{q}^{\prime}\left(f_{11} E_{q}^{\prime}+f_{12} \cos (\delta-\alpha)+f_{13} \sin (\delta-\alpha)+g_{11} E_{F D}\right) \\
& +f_{22}\left(f_{11} E_{q}^{\prime}+f_{12} \cos (\delta-\alpha)+f_{13} \sin (\delta-\alpha)+g_{11} E_{F D}\right) \cos (\delta-\alpha) \\
& -f_{22} E_{q}^{\prime} \sin (\delta-\alpha)(\omega-1)+f_{23}\left(f_{11} E_{q}^{\prime}+f_{12} \cos (\delta-\alpha)+f_{13} \sin (\delta-\alpha)+g_{11} E_{F D}\right) \sin (\delta-\alpha) \\
& +f_{23} E_{q}^{\prime} \cos (\delta-\alpha)(\omega-1)+f_{24} \cos ^{2}(\delta-\alpha)(\omega-1)-f_{24} \sin ^{2}(\delta-\alpha)(\omega-1) \\
& -2 f_{25} \cos (\delta-\alpha) \sin (\delta-\alpha)(\omega-1)+2 f_{26} \cos (\delta-\alpha) \sin (\delta-\alpha)(\omega-1) \\
& +f_{27}\left(f_{21} E_{q}^{\prime 2}+f_{22} E_{q}^{\prime} \cos (\delta-\alpha)+f_{23} E_{q}^{\prime} \sin (\delta-\alpha)+f_{24} \sin (\delta-\alpha) \cos (\delta-\alpha)\right. \\
& \left.+f_{25} \cos ^{2}(\delta-\alpha)+f_{26} \sin ^{2}(\delta-\alpha)+f_{27} \omega+f_{28} T_{m}\right) \\
& +f_{28}\left(f_{41} T_{m}+f_{42} G_{V}\right)
\end{aligned}
$$

On rearranging and simplifying Equation (8.26) we get

$$
\begin{aligned}
\dot{z}_{3}= & \left(2 f_{11} f_{21}+f_{27} f_{21}\right) E_{q}^{\prime 2} \\
& +\left(2 f_{21} f_{12}+f_{22} f_{11}-f_{23}+f_{27} f_{22}\right) E_{q}^{\prime} \cos (\delta-\alpha) \\
& +\left(2 f_{21} f_{13}+f_{22}+f_{23} f_{11}+f_{27} f_{23}\right) E_{q}^{\prime} \sin (\delta-\alpha) \\
& +\left(f_{22} f_{12}-f_{24}+f_{27} f_{25}\right) \cos ^{2}(\delta-\alpha) \\
& +\left(f_{23} f_{13}+f_{24}+f_{27} f_{26}\right) \sin ^{2}(\delta-\alpha) \\
& +\left(f_{22} f_{13}+f_{23} f_{12}+2 f_{25}-2 f_{26}+f_{27} f_{24}\right) \sin (\delta-\alpha) \cos (\delta-\alpha) \\
& +f_{27}^{2} \omega+\left(f_{27} f_{28}+f_{28} f_{41}\right) T_{m}+f_{28} f_{42} G_{v} \\
& +\left(f_{23}\right) E_{q}^{\prime} \omega \cos (\delta-\alpha) \\
& +\left(-f_{22}\right) E_{q}^{\prime} \omega \sin (\delta-\alpha) \\
& +\left(f_{24}\right) \omega \cos ^{2}(\delta-\alpha) \\
& +\left(-f_{24}\right) \omega \sin ^{2}(\delta-\alpha) \\
& +\left(-2 f_{25}+2 f_{26}\right) \omega \sin (\delta-\alpha) \cos (\delta-\alpha) \\
& +\left(2 f_{21} g_{11} E_{q}^{\prime}+f_{22} g_{11} \cos (\delta-\alpha)+f_{23} g_{11} \sin (\delta-\alpha)\right) E_{F D}
\end{aligned}
$$

For simplification let us denote

$$
\begin{aligned}
& 2 f_{11} f_{21}+f_{27} f_{21}=p_{31}, \\
& 2 f_{21} f_{12}+f_{22} f_{11}-f_{23}+f_{27} f_{22}=p_{32}, \\
& 2 f_{21} f_{13}+f_{22}+f_{23} f_{11}+f_{27} f_{23}=p_{33}, \\
& f_{22} f_{12}-f_{24}+f_{27} f_{25}=p_{34}, \\
& f_{23} f_{13}+f_{24}+f_{27} f_{26}=p_{35}, \\
& f_{22} f_{13}+f_{23} f_{12}+2 f_{25}-2 f_{26}+f_{27} f_{24}=p_{36}, \\
& f_{27}^{2}=p_{37}, \quad f_{27} f_{28}+f_{28} f_{41}=p_{38}, \\
& f_{28} f_{42}=p_{39}, \quad f_{23}=q_{31}, \quad-f_{22}=q_{32}, \\
& f_{24}=q_{33}, \quad-f_{24}=q_{34}, \quad-2 f_{25}+2 f_{26}=q_{35}, \\
& 2 f_{21} g_{11}=r_{31}, \quad f_{22} g_{11}=r_{32}, \quad f_{23} g_{11}=r_{33}
\end{aligned}
$$


On substituting Equation (8.28) in Equation (8.27) we get

$$
\begin{aligned}
\dot{z}_{3}= & p_{31}{E_{q}^{\prime}}^{2}+p_{32} E_{q}^{\prime} \cos (\delta-\alpha)+p_{33} E_{q}^{\prime} \sin (\delta-\alpha)+p_{34} \cos ^{2}(\delta-\alpha)+p_{35} \sin ^{2}(\delta-\alpha) \\
& +p_{36} \sin (\delta-\alpha) \cos (\delta-\alpha)+p_{37} \omega+p_{38} T_{m}+p_{39} G_{v} \\
& +q_{31} E_{q}^{\prime} \omega \cos (\delta-\alpha)+q_{32} E_{q}^{\prime} \omega \sin (\delta-\alpha)+q_{33} \omega \cos ^{2}(\delta-\alpha) \\
& +q_{34} \omega \sin ^{2}(\delta-\alpha)+q_{35} \omega \sin (\delta-\alpha) \cos (\delta-\alpha) \\
& +\left(r_{31} E_{q}^{\prime}+r_{32} \cos (\delta-\alpha)+r_{33} \sin (\delta-\alpha)\right) E_{F D}
\end{aligned}
$$

From Equation (8.24) we have

$$
\dot{z}_{3}=\sigma_{1}(x)+\gamma_{1}(x) E_{F D}=v_{1}
$$

Thus, equating Equation (8.29) and Equation (8.30) we get

$$
\begin{aligned}
\sigma_{1}(x)= & p_{31} E_{q}^{\prime 2}+p_{32} E_{q}^{\prime} \cos (\delta-\alpha)+p_{33} E_{q}^{\prime} \sin (\delta-\alpha)+p_{34} \cos ^{2}(\delta-\alpha)+p_{35} \sin ^{2}(\delta-\alpha) \\
& +p_{36} \sin (\delta-\alpha) \cos (\delta-\alpha)+p_{37} \omega+p_{38} T_{m}+p_{39} G_{v} \\
& +q_{31} E_{q}^{\prime} \omega \cos (\delta-\alpha)+q_{32} E_{q}^{\prime} \omega \sin (\delta-\alpha)+q_{33} \omega \cos ^{2}(\delta-\alpha) \\
& +q_{34} \omega \sin ^{2}(\delta-\alpha)+q_{35} \omega \sin (\delta-\alpha) \cos (\delta-\alpha) \\
\text { i.e } \quad \sigma_{1}(x)= & p_{31} x_{1}^{2}+p_{32} x_{1} \cos \left(x_{3}-\alpha\right)+p_{33} x_{1} \sin \left(x_{3}-\alpha\right)+p_{34} \cos ^{2}\left(x_{3}-\alpha\right)+p_{35} \sin ^{2}\left(x_{3}-\alpha\right) \\
& +p_{36} \sin \left(x_{3}-\alpha\right) \cos \left(x_{3}-\alpha\right)+p_{37} x_{2}+p_{38} x_{4}+p_{39} x_{5} \\
& +q_{31} x_{1} x_{2} \cos \left(x_{3}-\alpha\right)+q_{32} x_{1} x_{2} \sin \left(x_{3}-\alpha\right)+q_{33} x_{2} \cos ^{2}\left(x_{3}-\alpha\right) \\
& +q_{34} x_{2} \sin ^{2}\left(x_{3}-\alpha\right)+q_{35} x_{2} \sin \left(x_{3}-\alpha\right) \cos \left(x_{3}-\alpha\right) \\
\text { i.e } \quad \gamma_{1}(x)= & r_{31} x_{1}+r_{32} \cos \left(x_{3}-\alpha\right)+r_{33} \sin \left(x_{3}-\alpha\right)
\end{aligned}
$$

Thus, using Equation (8.30) and Equation (8.31) we can compute the excitation field EMF $E_{F D}$ as

$$
\begin{aligned}
& E_{F D}=\gamma_{1}^{-1}(x)\left(v_{1}-\sigma_{1}(x)\right) \\
& E_{F D}=\alpha_{1}(x)+\beta_{1}(x) v_{1}
\end{aligned}
$$

where $\alpha_{1}(x)=-\gamma_{1}^{-1}(x) \sigma_{1}(x)$ and $\beta_{1}(x)=\gamma_{1}^{-1}(x)$. From Equation (8.22) and Equation (8.24), i.e. by taking the derivative of $\dot{T}_{m}$ we have

$$
\begin{aligned}
\dot{z}_{5}=\ddot{T}_{m} & =f_{41} \dot{T}_{m}+f_{42} \dot{G}_{v} \\
& =f_{41}\left(f_{41} T_{m}+f_{42} G_{v}\right)+f_{42}\left(f_{51} \omega+f_{52} G_{v}+g_{55} u_{T}\right) \\
& =f_{42} f_{51} \omega+f_{41}^{2} T_{m}+\left(f_{41} f_{42}+f_{42} f_{52}\right) G_{v}+f_{42} g_{55} u_{T}
\end{aligned}
$$

Let us denote $f_{42} f_{51}=p_{51}, f_{41}^{2}=p_{52}, f_{41} f_{42}+f_{42} f_{52}=p_{53}$, and $f_{42} g_{55}=r_{51}$. Thus, Equation (8.33) can be simplified as

$$
\dot{z}_{5}=\ddot{T}_{m}=p_{51} \omega+p_{52} T_{m}+p_{53} G_{v}+r_{51} u_{T}
$$

From Equation (8.24) we have

$$
\dot{z}_{5}=\sigma_{2}(x)+\gamma_{2}(x) u_{T}=v_{2}
$$

Thus, equating Equation (8.34) and Equation (8.35) we get

$$
\begin{aligned}
\sigma_{2}(x) & =p_{51} \omega+p_{52} T_{m}+p_{53} G_{v} \\
\text { i.e } \quad \sigma_{2}(x) & =p_{51} x_{2}+p_{52} x_{4}+p_{53} x_{5} \\
\gamma_{2}(x) & =r_{51}
\end{aligned}
$$


Thus, using Equation (8.35) and Equation (8.36) we can compute the turbine valve control $u_{T}$ as

$$
\begin{aligned}
& u_{T}=\gamma_{2}^{-1}(x)\left(v_{2}-\sigma_{2}(x)\right) \\
& u_{T}=\alpha_{2}(x)+\beta_{2}(x) v_{2}
\end{aligned}
$$

where $\alpha_{2}(x)=-\gamma_{2}^{-1}(x) \sigma_{2}(x)$ and $\beta_{2}(x)=\gamma_{2}^{-1}(x)$.

We next design the linear controllers $v_{1}$ and $v_{2}$. The purpose of the linear controllers $v_{1}$ and $v_{2}$ is to regulate the state variables $\delta$ and $T_{m}$ to their set points. The set points for the remaining state variables are found through the equilibrium condition $\delta(t)=1$ radian, and $T_{m}(t)=1.0012$ p.u.. Application of the state feedback control $u_{1}=E_{F D}=\alpha_{1}(x)+\beta_{1}(x) v_{1}, u_{2}=u_{T}=\alpha_{2}(x)+\beta_{2}(x) v_{2}$, and the state transformation $z=T(x)$ to the nonlinear model of the system, results in a linear model of the system in the new coordinates as

$$
\dot{\mathbf{z}}=\mathbf{A z}+\mathbf{B v}
$$

where

$$
\begin{aligned}
\mathbf{z}^{T}= & {\left[\begin{array}{lllll}
z_{1} & z_{2} & z_{3} & z_{4} & z_{5}
\end{array}\right] } \\
\mathbf{v}= & {\left[\begin{array}{l}
v_{1} \\
v_{2}
\end{array}\right] } \\
\mathbf{A}= & {\left[\begin{array}{lllll}
0 & 1 & 0 & 0 & 0 \\
0 & 0 & 1 & 0 & 0 \\
0 & 0 & 0 & 0 & 0 \\
0 & 0 & 0 & 0 & 1 \\
0 & 0 & 0 & 0 & 0
\end{array}\right] } \\
& \mathbf{B}=\left[\begin{array}{ll}
0 & 0 \\
0 & 0 \\
1 & 0 \\
0 & 0 \\
0 & 1
\end{array}\right]
\end{aligned}
$$

The linear controller $\mathbf{v}=-K \mathbf{z}$ can be designed either by pole placement or LQR technique such that $A-B K$ is Hurwitz. We use the LQR technique to design the controller gain matrix $K$. Thus, by using the feedback law,

$$
\mathbf{v}=-K \mathbf{z}
$$

the weighting matrices

$$
Q=\left[\begin{array}{ccccc}
300 & 0 & 0 & 0 & 0 \\
0 & 250 & 0 & 0 & 0 \\
0 & 0 & 200 & 0 & 0 \\
0 & 0 & 0 & 200 & 0 \\
0 & 0 & 0 & 0 & 250
\end{array}\right]
$$

and

$$
R=\left[\begin{array}{cc}
0.07 & 0 \\
0 & 0.07
\end{array}\right]
$$

and the state space matrices $(A, B)$ as given in Equation (8.40) and Equation (8.41) the control gain $K$ is found to be

$$
K=\left[\begin{array}{ccccc}
65.4654 & 104.0206 & 55.3641 & 0 & 0 \\
0 & 0 & 0 & 53.4522 & 60.6493
\end{array}\right]
$$

Thus, we have

$$
\begin{aligned}
v_{1} & =-K_{11}\left(z_{1}-z_{1 d}\right)-K_{12}\left(z_{2}-z_{2 d}\right)-K_{13}\left(z_{3}-z_{3 d}\right) \\
& =-K_{11}\left(\delta-\delta_{d}\right)-K_{12}\left(\dot{\delta}-\dot{\delta}_{d}\right)-K_{13}\left(\ddot{\delta}-\ddot{\delta}_{d}\right)
\end{aligned}
$$


and

$$
\begin{aligned}
v_{2} & =-K_{24}\left(z_{4}-z_{4 d}\right)-K_{25}\left(z_{5}-z_{5 d}\right) \\
& =-K_{24}\left(T_{m}-T_{m d}\right)-K_{25}\left(\dot{T}_{m}-\dot{T}_{m d}\right)
\end{aligned}
$$

where $z_{1 d}, z_{2 d}, z_{3 d}, z_{4 d}, z_{5 d}$ are the desired values of the new state variables. Since we want the set point to be an equilibrium, all the derivatives of $z_{1}$ and $z_{4}$ have to be zero. Thus $z_{2 d}=z_{3 d}=z_{5 d}=0$. Also $z_{1 d}=1$ radian, and $z_{4 d}=1.0012 \mathrm{p}$.u. are appropriately chosen within a reasonable physical limit of the system. The limits of the excitation field voltage of the generator are given by $E_{F D(\max )}=5$ p.u. and $E_{F D(\min )}=-5$ p.u. The limits of the turbine gate opening are given by $G_{V(\max )}=1.5 \mathrm{p} . \mathrm{u}$. and $G_{V(\min )}=$ 0 p.u. The operating conditions of the system are as given in example 3.4 with $I_{F 0}=1.6315, I_{q 0}=0.4047$, $I_{d 0}=-0.9185, V_{q 0}=0.9670, V_{d 0}=-0.6628, V_{\infty}=1.00, V_{t 0}=1.172, \delta_{0}-\alpha=53.736^{\circ}, E_{q 0}^{\prime}=1.1925$, and $\tau_{d 0}^{\prime}=5.90$

From Figure 8.1 we can see that the state variables $E_{q}^{\prime}, \omega, \delta, T_{m}$, and $G_{V}$ attain their respective steady state values in approximately 5 to 7 seconds. Figure 8.2 shows that the generator terminal voltage $V_{t}$ reaches a steady state value of $1.172 \mathrm{p} . \mathrm{u}$. which is equal to the desired steady state operating point $V_{t 0}=1.172$, in approximately 5 seconds. Figure 8.3 shows that the rotor angle $\delta$ settles to a steady state value of approximately 1 radians, which is equal to the steady state operating condition $\delta_{0}=1$ radian, in approximately 7 seconds. The angular velocity or the frequency $\omega$ of the synchronous generator settles to a steady state value of $1 \mathrm{rad} / \mathrm{sec}$ which is equal to the desired operating condition $\omega_{0}=1 \mathrm{rad} / \mathrm{sec}$ which can be verified from Figure 8.4. Also, from Figure 8.5 and Figure 8.6 we can see that the control inputs, $E_{F D}$ and $u_{T}$, settle to their steady state values of 2.529 p.u. and $1.0512 \mathrm{p}$.u. respectively. To verify our simulation results we analytically compute the steady state equilibrium values of the original state variables and the control inputs. The steady state equilibrium in the original co-ordinates can be computed by solving the differential equation, $\dot{x}=f(x)+g(x) u=0$. Thus using Equation (8.22) we can write

$$
\begin{aligned}
& \dot{E}_{q}^{\prime}=f_{11} E_{q}^{\prime}+f_{12} \cos (\delta-\alpha)+f_{13} \sin (\delta-\alpha)+g_{11} E_{F D}=0 \\
& \dot{\omega}=f_{21} E_{q}^{\prime 2}+f_{22} E_{q}^{\prime} \cos (\delta-\alpha)+f_{23} E_{q}^{\prime} \sin (\delta-\alpha)+f_{24} \sin (\delta-\alpha) \cos (\delta-\alpha) \\
& \quad+f_{25} \cos ^{2}(\delta-\alpha)+f_{26} \sin ^{2}(\delta-\alpha)+f_{27} \omega+f_{28} T_{m}=0 \\
& \dot{\delta}=\omega-1=0 \\
& \dot{T}_{m}=f_{41} T_{m}+f_{42} G_{V}=0 \\
& \dot{G}_{V}=f_{51} \omega+f_{52} G_{V}+g_{55} u_{T}=0
\end{aligned}
$$

The steady state equilibrium in the original co-ordinates $E_{q}^{\prime}, \omega, \delta, T_{m}$, and $G_{V}$ can be found by using $z_{1 d}=\delta=1$ radian, and $z_{4 d}=T_{m}=1.0012$ p.u., and solving the differential equation

$$
\begin{aligned}
\dot{\omega}= & f_{21} E_{q}^{\prime 2}+f_{22} E_{q}^{\prime} \cos (\delta-\alpha)+f_{23} E_{q}^{\prime} \sin (\delta-\alpha)+f_{24} \sin (\delta-\alpha) \cos (\delta-\alpha) \\
& \quad+f_{25} \cos ^{2}(\delta-\alpha)+f_{26} \sin ^{2}(\delta-\alpha)+f_{27} \omega+f_{28} T_{m}=0 \\
\dot{\delta}= & \omega-1=0 \\
\dot{T}_{m}= & f_{41} T_{m}+f_{42} G_{V}=0
\end{aligned}
$$

Also the steady state equilibrium of the control inputs $E_{F D}$ and $u_{T}$ can be found by using $\dot{E}_{q}^{\prime}=0$, and $\dot{G}_{V}=0$, from Equation (8.48), and the steady state equilibrium values of the original state variables $E_{q(s s)}^{\prime}$, $\omega_{(s s)}, \delta_{(s s)}, T_{m(s s)}$, and $G_{V(s s)}$ obtained by solving Equation (8.49) i.e.

$$
\begin{aligned}
0 & =f_{11} E_{q 0}^{\prime}+f_{12} \cos \left(\delta_{0}-\alpha\right)+f_{13} \sin \left(\delta_{0}-\alpha\right)+g_{11} E_{F D(s s)} \\
& =-0.5517 \times 1.1925+0.3822 \times 0.5915+0.0037 \times 0.8063+0.1695 \times E_{F D(s s)} \\
0 & =f_{51} \omega_{0}+f_{52} G_{V 0}+g_{55} u_{T(s s)} \\
& =-0.25 \times 1-5 \times 1.0012+5 \times u_{T(s s)}
\end{aligned}
$$

Solving Equation (8.50) we get $E_{F D(s s)}=2.529$ and $u_{T(s s)}=1.0512$, which are in agreement with our simulation results. 


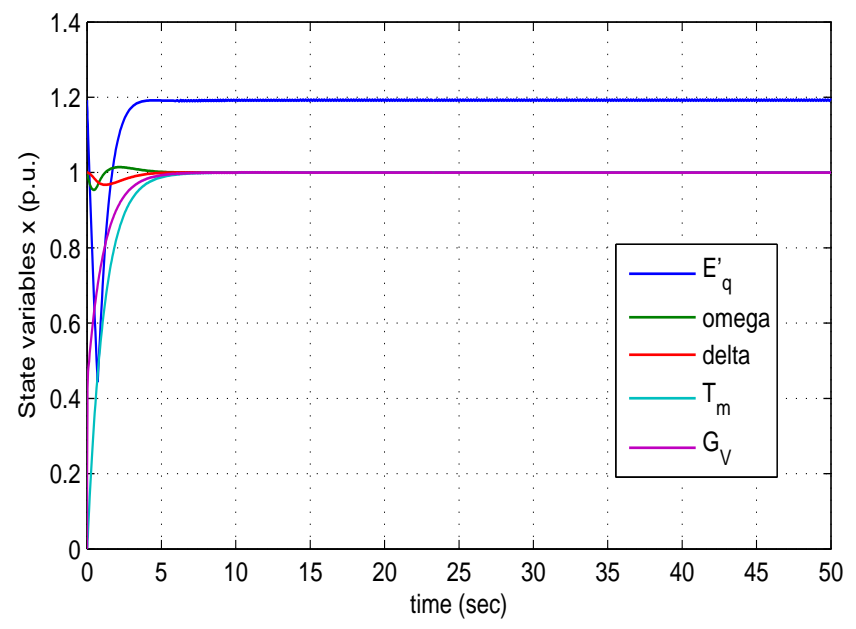

Figure 8.1: Plot of the state variables $E_{q}^{\prime}, \omega, \delta, T_{m}$, and $G_{V}$ vs time for the input-state nonlinear feedback linearizing controller applied to the reduced order model

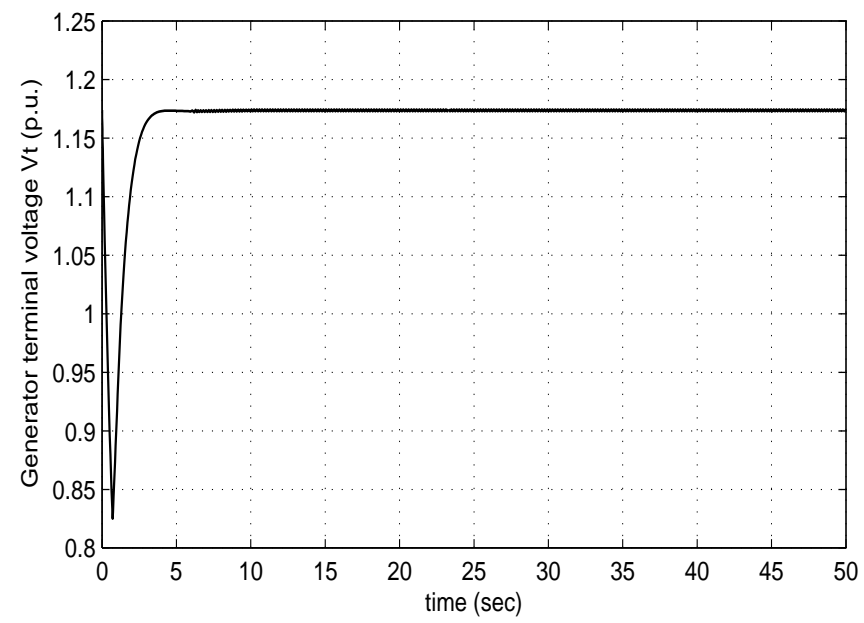

Figure 8.2: Plot of the generator terminal voltage $V_{t}$ vs time for the input-state nonlinear feedback linearizing controller applied to the reduced order model

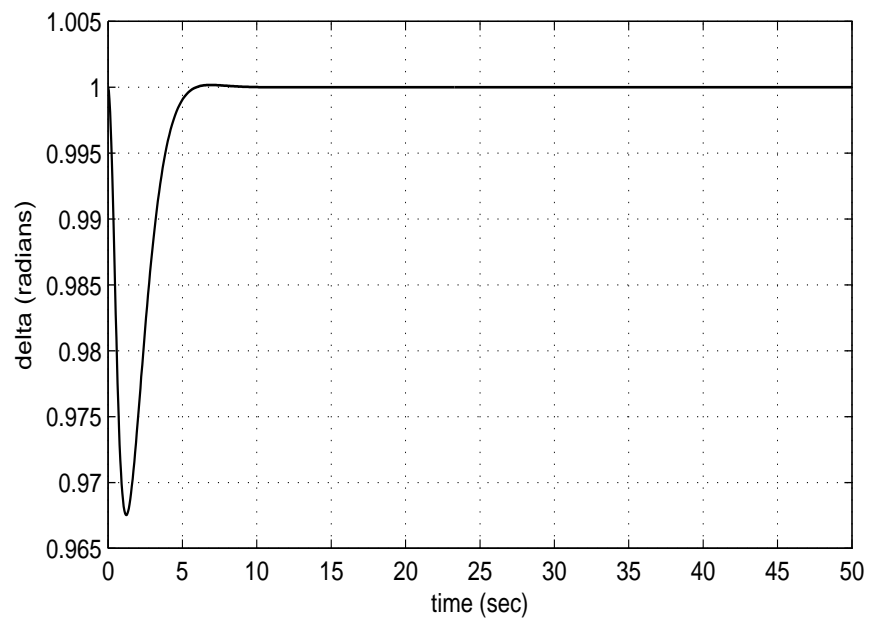

Figure 8.3: Plot of $\delta$ vs time for the input-state nonlinear feedback linearizing controller applied to the reduced order model 


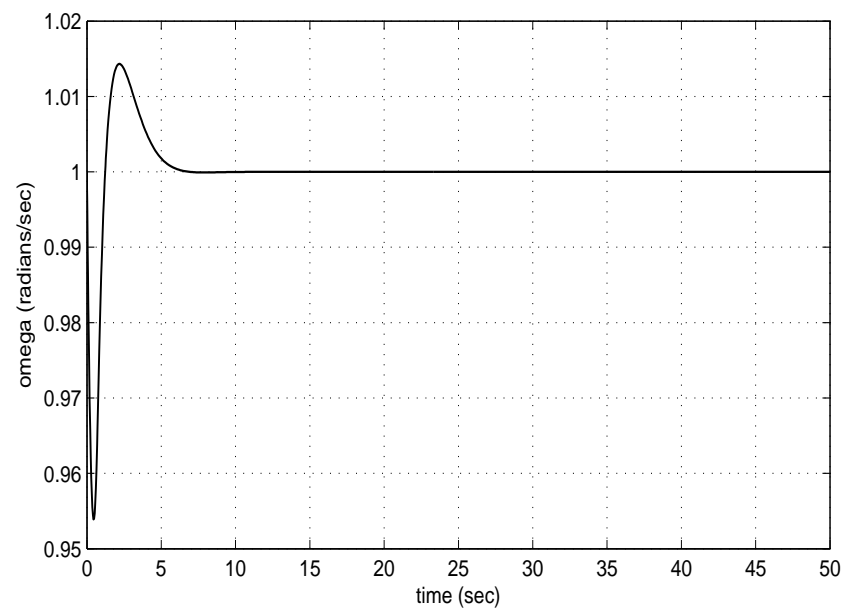

Figure 8.4: Plot of $\omega$ vs time for the input-state nonlinear feedback linearizing controller applied to the reduced order model

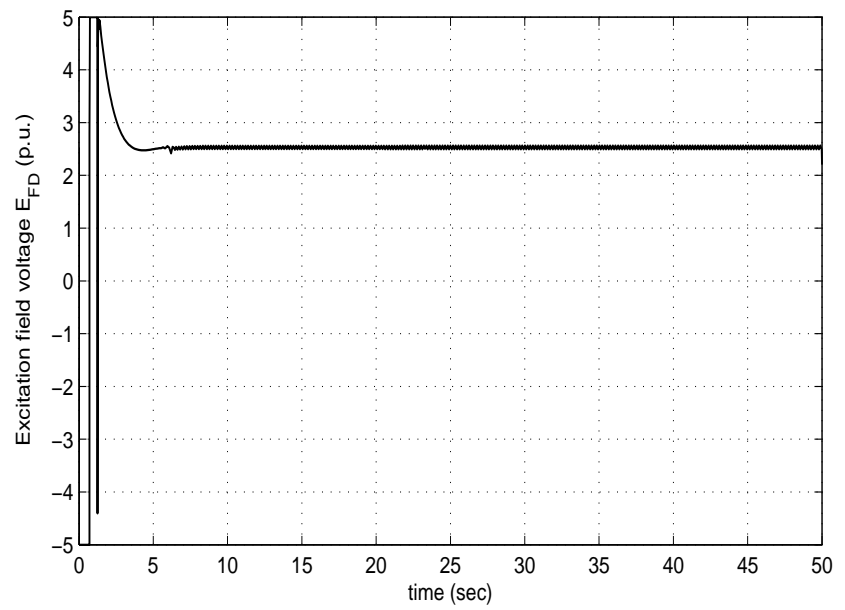

Figure 8.5: Plot of the generator excitation field control $E_{F D}$ vs time for the input-state nonlinear feedback linearizing controller applied to the reduced order model

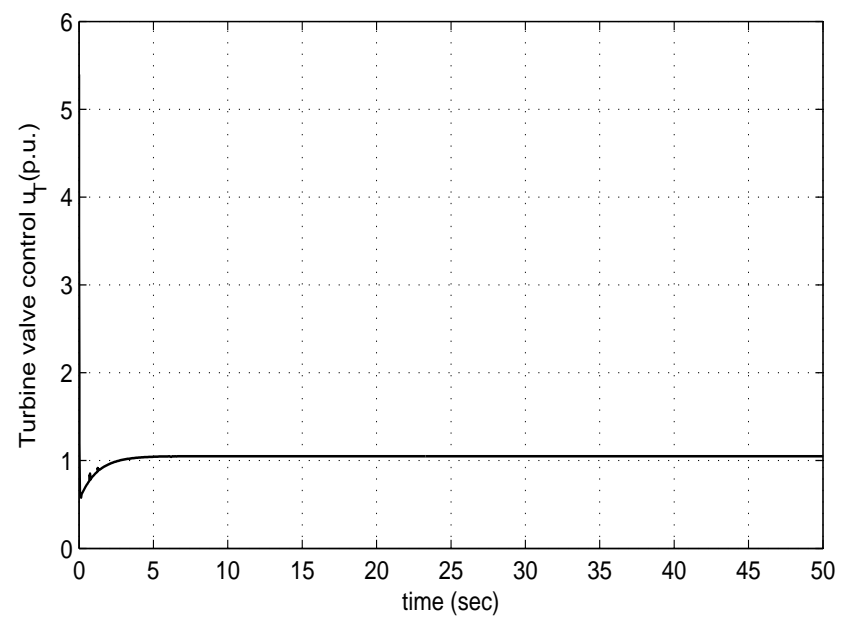

Figure 8.6: Plot of the turbine valve control $u_{T}$ vs time for the input-state nonlinear feedback linearizing controller applied to the reduced order model 


\subsection{Simulation Results for the Nonlinear Feedback Linearizing Controller applied to the Truth Model}

For the reduced order nonlinear model the state variables are $x=\left[E_{q}^{\prime}, \omega, \delta, T_{m}, G_{V}\right]^{\mathrm{T}}$, the two control inputs are $u=\left[E_{F D}, u_{T}\right]^{\mathrm{T}}$, and the two regulated outputs are $y=\left[V_{t}, \omega\right]^{\mathrm{T}}$. Whereas, for the truth model $x=\left[I_{d}, I_{F}, I_{D}, I_{q}, I_{Q}, \omega, \delta, T_{m}, G_{V}\right]^{\mathrm{T}}$ is the vector of state variables, $u=\left[V_{F}, u_{T}\right]^{\mathrm{T}}$ is vector of control inputs, and $y=\left[V_{t}, \omega\right]^{\mathrm{T}}$ is the vector of outputs. Thus we can see that the first control input $V_{F}$ of the truth model and the first control input $E_{F D}$ of the reduced order model are different. As given in Equation (10.41) the excitation field emf, $E_{F D}$, which is the first control input for the reduced order nonlinear model is related to field voltage, $V_{F}$, which is the first control input for the truth model by the following expression

$$
\left(\frac{V_{F}}{r_{F}}\right) \omega_{R} k M_{F}=E_{F D}
$$

Equation (8.51) can be rearranged to get the following expression

$$
\begin{aligned}
V_{F} & =\left(\frac{r_{F}}{\omega_{R} k M_{F}}\right) E_{F D} \\
& =e_{15} E_{F D}
\end{aligned}
$$

In the above expression, $\omega_{R}=1$ p.u., and $e_{15}=\left(\frac{r_{F}}{\omega_{R} k M_{F}}\right)$. Also there is a non physical state $E_{q}^{\prime}$ in the reduced order model that needs to be reconstructed from the states of the truth model.

The nonlinear feedback linearizing controller that was designed for the reduced order nonlinear model is now tested on the truth model. From Equation (8.32) the excitation field EMF, $E_{F D}$, is computed as

$$
\begin{aligned}
& E_{F D}=\gamma_{1}^{-1}(x)\left(v_{1}-\sigma_{1}(x)\right) \\
& E_{F D}=\alpha_{1}(x)+\beta_{1}(x) v_{1}
\end{aligned}
$$

where $\alpha_{1}(x)=-\gamma_{1}^{-1}(x) \sigma_{1}(x) ; \beta_{1}(x)=\gamma_{1}^{-1}(x)$ and

$$
\begin{aligned}
\sigma_{1}(x)= & p_{31} E_{q}^{\prime 2}+p_{32} E_{q}^{\prime} \cos (\delta-\alpha)+p_{33} E_{q}^{\prime} \sin (\delta-\alpha)+p_{34} \cos ^{2}(\delta-\alpha)+p_{35} \sin ^{2}(\delta-\alpha) \\
& +p_{36} \sin (\delta-\alpha) \cos (\delta-\alpha)+p_{37} \omega+p_{38} T_{m}+p_{39} G_{v} \\
& +q_{31} E_{q}^{\prime} \omega \cos (\delta-\alpha)+q_{32} E_{q}^{\prime} \omega \sin (\delta-\alpha)+q_{33} \omega \cos ^{2}(\delta-\alpha) \\
& +q_{34} \omega \sin ^{2}(\delta-\alpha)+q_{35} \omega \sin (\delta-\alpha) \cos (\delta-\alpha) \\
\text { i.e } \quad \sigma_{1}(x)= & p_{31} x_{1}^{2}+p_{32} x_{1} \cos \left(x_{3}-\alpha\right)+p_{33} x_{1} \sin \left(x_{3}-\alpha\right)+p_{34} \cos ^{2}\left(x_{3}-\alpha\right)+p_{35} \sin ^{2}\left(x_{3}-\alpha\right) \\
& +p_{36} \sin \left(x_{3}-\alpha\right) \cos \left(x_{3}-\alpha\right)+p_{37} x_{2}+p_{38} x_{4}+p_{39} x_{5} \\
& +q_{31} x_{1} x_{2} \cos \left(x_{3}-\alpha\right)+q_{32} x_{1} x_{2} \sin \left(x_{3}-\alpha\right)+q_{33} x_{2} \cos ^{2}\left(x_{3}-\alpha\right) \\
& +q_{34} x_{2} \sin ^{2}\left(x_{3}-\alpha\right)+q_{35} x_{2} \sin \left(x_{3}-\alpha\right) \cos \left(x_{3}-\alpha\right) \\
\text { i.e } \quad \gamma_{1}(x)= & r_{31} x_{1}+r_{32} \cos \left(x_{3}-\alpha\right)+r_{33} \sin \left(x_{3}-\alpha\right)
\end{aligned}
$$

From Equation (8.52) the field voltage $V_{F}$ can be written as

$$
V_{F}=\left(\frac{r_{F}}{\omega_{R} k M_{F}}\right) E_{F D}=\left(\frac{r_{F}}{\omega_{R} k M_{F}}\right) \gamma_{1}^{-1}(x)\left(v_{1}-\sigma_{1}(x)\right)=e_{15}\left(\alpha_{1}(x)+\beta_{1}(x) v_{1}\right)
$$

In Equation (8.55), $V_{F}$ depends on $\sigma_{1}(x)$ and $\gamma_{1}(x)$ which depend on the fictitious state variable $E_{q}^{\prime}$, which is not a physical quantity that can be measured or a state variable of the truth model. Since $E_{q}^{\prime}$ cannot be measured using sensors, we cannot apply the field voltage $V_{F}$ directly on the truth model without eliminating the state variable $E_{q}^{\prime}$ in the above expression. This problem can be solved by expressing $E_{q}^{\prime}$ as a function of 
any of the measurable states of the truth model. From Equation (10.136) as given in the derivation of the reduced order model in the appendix we have

$$
E=E_{q}^{\prime}-\left(L_{d}-L_{d}^{\prime}\right) I_{d}
$$

Substituting $I_{d}$ from Equation (3.8) in the above equation

$$
E=E_{q}^{\prime}-\left(L_{d}-L_{d}^{\prime}\right)\left(\frac{-\left(E_{q}^{\prime}-V_{\infty q}\right)\left(L_{q}+L_{e}\right)-V_{\infty d}\left(r+R_{e}\right)}{\left(r+R_{e}\right)^{2}+\left(L_{d}^{\prime}+L_{e}\right)\left(L_{q}+L_{e}\right)}\right)
$$

For simplification of the above expression let us denote $L_{d}-L_{d}^{\prime}=L_{2}, L_{q}+L_{e}=L_{1}, r+R_{e}=R_{1}$, $\left(r+R_{e}\right)^{2}+\left(L_{d}^{\prime}+L_{e}\right)\left(L_{q}+L_{e}\right)=M_{1}$. Thus Equation (8.57) can be written as

$$
E=E_{q}^{\prime}-L_{2}\left(\frac{-\left(E_{q}^{\prime}-V_{\infty q}\right) L_{1}-V_{\infty d} R_{1}}{M_{1}}\right)
$$

The above expression can be simplified to get

$$
E=\left(1+\frac{L_{1} L_{2}}{M_{1}}\right) E_{q}^{\prime}-\left(\frac{L_{1} L_{2} V_{\infty}}{M_{1}}\right) \cos (\delta-\alpha)-\left(\frac{R_{1} L_{2} V_{\infty}}{M_{1}}\right) \sin (\delta-\alpha)
$$

Let us denote

$$
\begin{aligned}
& \left(1+\frac{L_{1} L_{2}}{M_{1}}\right)=e_{11} \\
& \left(\frac{L_{1} L_{2} V_{\infty}}{M_{1}}\right)=e_{12} \\
& \left(\frac{R_{1} L_{2} V_{\infty}}{M_{1}}\right)=e_{13}
\end{aligned}
$$

Substituting Equation (8.60) in Equation (8.59) and rearranging, $E_{q}^{\prime}$ can be written as

$$
E_{q}^{\prime}=\frac{1}{e_{11}} E+\frac{e_{12}}{e_{11}} \cos (\delta-\alpha)+\frac{e_{13}}{e_{11}} \sin (\delta-\alpha)
$$

From Equation (10.39) the excitation field emf, $E$, is related to the field current $I_{F}$ as

$$
E=\omega_{R} k M_{F} I_{F}=e_{14} I_{F}
$$

Substituting Equation (8.62) in Equation (8.61)

$$
E_{q}^{\prime}=\frac{e_{14}}{e_{11}} I_{F}+\frac{e_{12}}{e_{11}} \cos (\delta-\alpha)+\frac{e_{13}}{e_{11}} \sin (\delta-\alpha)
$$

$E_{q}^{\prime}$ is now expressed as a function of the field current $I_{F}$, and rotor angle $\delta$, which are state variables of the truth model. While the field current $I_{F}$ can be measured using a sensor, it is not always possible to measure the rotor angle $\delta$, using a sensor. In this case we assume that $\delta$ can be measured. Using Equation (8.56) and Equation (8.62), $E_{q}^{\prime}$ can also be written as

$$
E_{q}^{\prime}=e_{14} I_{F}+L_{2} I_{d}
$$

where $I_{F}$ and $I_{d}$ are both state variables of the truth model which can be measured using senors. Thus the first control input for the truth model which is the field voltage, $V_{F}$, as given in Equation (8.55) can be implemented on the truth model by using either Equation (8.63) or Equation (8.64) to eliminate $E_{q}^{\prime}$ in the expression for $V_{F}$. We prefer Equation (8.63) instead of Equation (8.64) since the rotor angle $\delta$, is a state variable in both the reduced order and the truth model, whereas the direct axis current $I_{d}$, is not a state 
variable in the reduced order model. From Equation (8.37) the second control input for the truth model which is the turbine valve control $u_{T}$, is given by

$$
\begin{aligned}
& u_{T}=\gamma_{2}^{-1}(x)\left(v_{2}-\sigma_{2}(x)\right) \\
& u_{T}=\alpha_{2}(x)+\beta_{2}(x) v_{2}
\end{aligned}
$$

where $\alpha_{2}(x)=-\gamma_{2}^{-1}(x) \sigma_{2}(x), \beta_{2}(x)=\gamma_{2}^{-1}(x)$ and

$$
\begin{aligned}
\sigma_{2}(x) & =p_{51} \omega+p_{52} T_{m}+p_{53} G_{v} \\
\text { i.e } \quad \sigma_{2}(x) & =p_{51} x_{2}+p_{52} x_{4}+p_{53} x_{5} \\
\gamma_{2}(x) & =r_{51}
\end{aligned}
$$

As seen earlier, in the section for nonlinear feedback linearizing controller design for the reduced order model, application of the state feedback control, $u_{1}=E_{F D}=\alpha_{1}(x)+\beta_{1}(x) v_{1}, u_{2}=u_{T}=\alpha_{2}(x)+\beta_{2}(x) v_{2}$, and the state transformation $z=T(x)$ to the reduced order nonlinear model of the system, results in a linear model of the system in the new coordinates. However, this is true only for the reduced order model, but not true for the truth model. The resulting closed loop system in the case of the truth model is not linear at all. The linear controllers $v_{1}$ and $v_{2}$ were designed by using the LQR technique for the reduced order nonlinear model. The gains of this linear LQR controller are tuned once again so that the nonlinear controller works efficiently on the truth model. Thus, by using the feedback law,

$$
\mathbf{v}=-K \mathbf{z}
$$

the weighting matrices

$$
Q=\left[\begin{array}{ccccc}
250 & 0 & 0 & 0 & 0 \\
0 & 250 & 0 & 0 & 0 \\
0 & 0 & 250 & 0 & 0 \\
0 & 0 & 0 & 250 & 0 \\
0 & 0 & 0 & 0 & 250
\end{array}\right]
$$

and

$$
R=\left[\begin{array}{cc}
30000 & 0 \\
0 & 30000
\end{array}\right]
$$

and the state space matrices $(A, B)$ as given in Equation (8.40)and Equation (8.41) the control gain $K$ is found to be

$$
K=\left[\begin{array}{ccccc}
0.0913 & 0.4201 & 0.9212 & 0 & 0 \\
0 & 0 & 0 & 0.0913 & 0.4369
\end{array}\right]
$$

Thus, we have

$$
\begin{aligned}
v_{1} & =-K_{11}\left(z_{1}-z_{1 d}\right)-K_{12}\left(z_{2}-z_{2 d}\right)-K_{13}\left(z_{3}-z_{3 d}\right) \\
& =-K_{11}\left(\delta-\delta_{d}\right)-K_{12}\left(\dot{\delta}-\dot{\delta}_{d}\right)-K_{13}\left(\ddot{\delta}-\ddot{\delta}_{d}\right)
\end{aligned}
$$

and

$$
\begin{aligned}
v_{2} & =-K_{24}\left(z_{4}-z_{4 d}\right)-K_{25}\left(z_{5}-z_{5 d}\right) \\
& =-K_{24}\left(T_{m}-T_{m d}\right)-K_{25}\left(\dot{T}_{m}-\dot{T}_{m d}\right)
\end{aligned}
$$

where $z_{1 d}, z_{2 d}, z_{3 d}, z_{4 d}, z_{5 d}$ are the desired values of the new state variables. Figure 8.7 to Figure 8.11 show simulation results for the nonlinear feedback linearizing controller applied to the truth model. From Figure 8.7, Figure 8.8, and Figure 8.9, we can see that $V_{t}, \omega$, and $\delta$ oscillate about their respective steady state values, where the oscillations slowly decay with time. Figure 8.10 and Figure 8.11 show the plots for the two control inputs, $V_{F}$ and $u_{T}$, respectively. The generator excitation voltage $V_{F}$ oscillates about its steady state value of 0.00121 p.u. and the turbine valve control settles to its steady state value of 1.0512 p.u. 


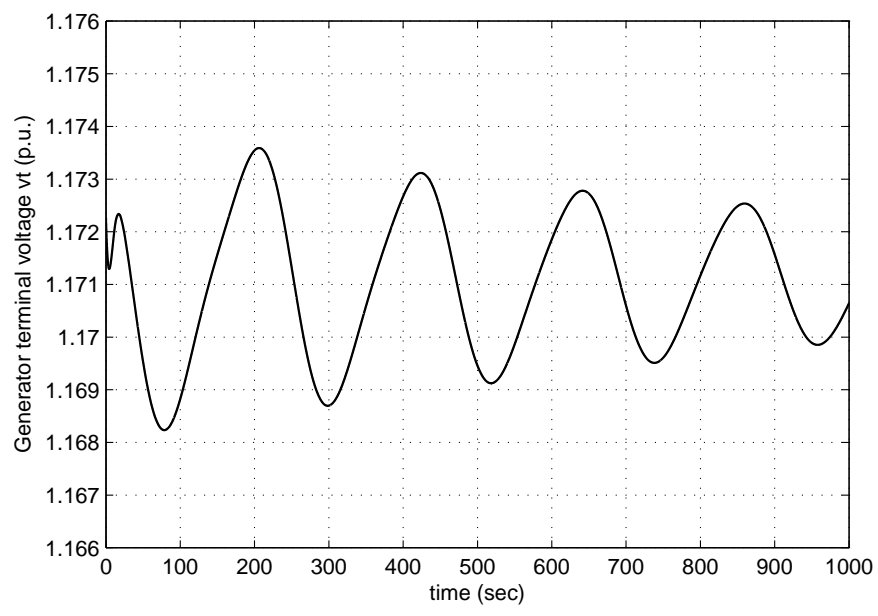

Figure 8.7: Plot of the generator terminal voltage $V_{t}$ vs time for the nonlinear feedback linearizing controller applied to the truth model

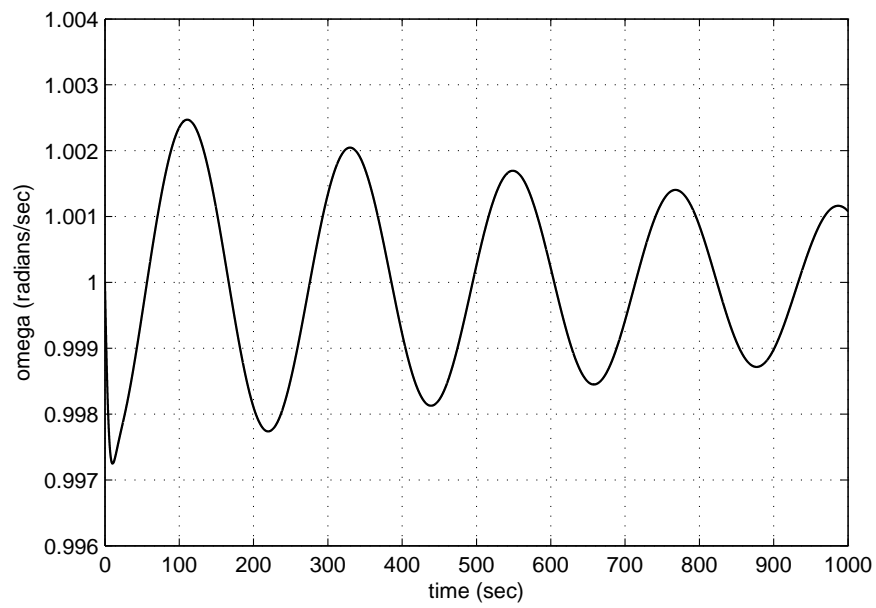

Figure 8.8: Plot of the angular velocity $\omega$ vs time for the nonlinear feedback linearizing controller applied to the truth model

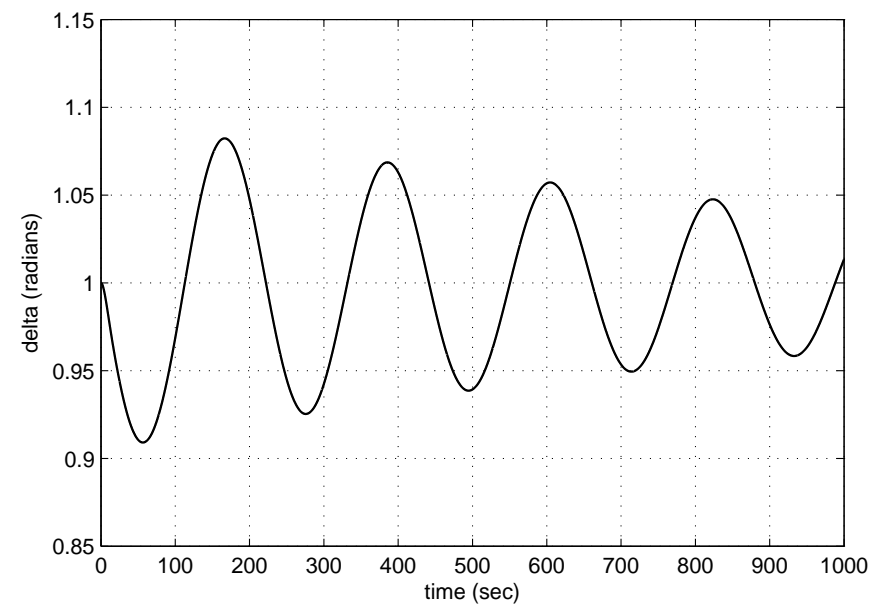

Figure 8.9: Plot of the rotor angle $\delta$ vs time for the nonlinear feedback linearizing controller applied to the truth model 


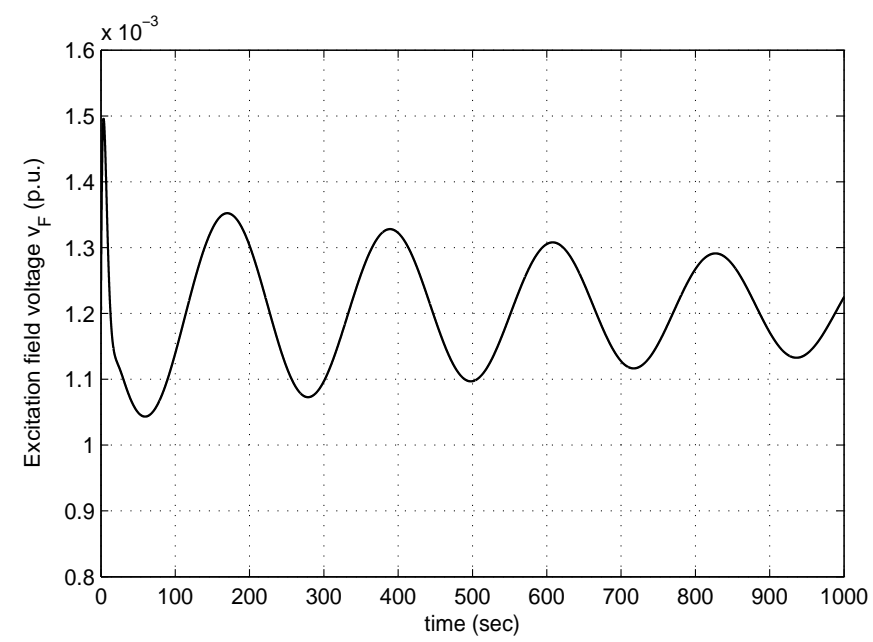

Figure 8.10: Plot of the generator excitation voltage $V_{F}$ vs time for the nonlinear feedback linearizing controller applied to the truth model

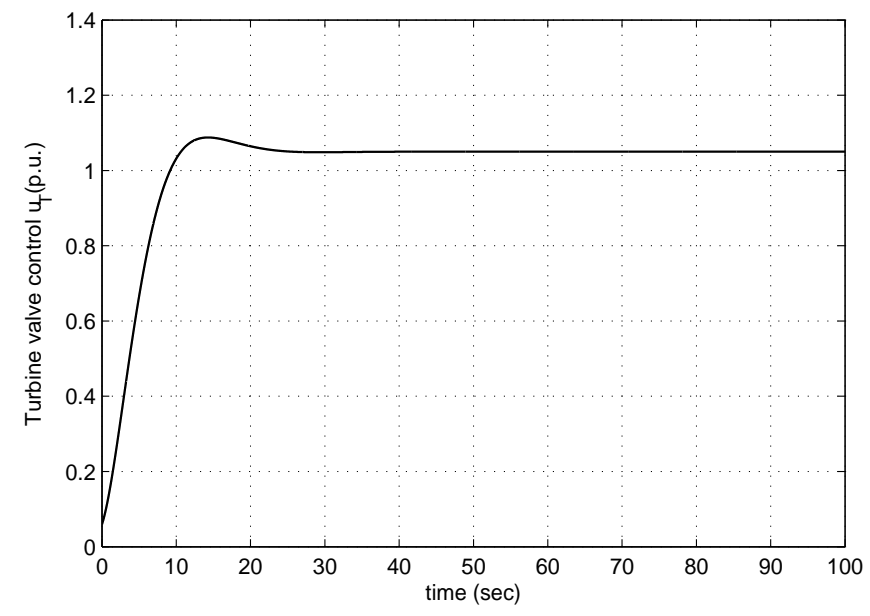

Figure 8.11: Plot of the turbine valve control $u_{T}$ vs time for the nonlinear feedback linearizing controller applied to the truth model 


\section{Simulation results for the Controllers at different Operating Points}

In this section we test the LTR-based LQG, nonlinear feedback linearizing controller, and the LQR-based full-state feedback controllers, which were tested on the truth model in the previous section, at new operating points. The operating points at which the controllers are tested are given in Table 9.1. Operating point $\mathrm{I}$, is the original desired operating point about which the reduced order model was linearized, and all the controllers were designed and tested in the previous sections. In this section we will test the controllers that we tested on the truth model, in the previous section at Operating Point I, at new operating points II and III respectively, without making any change to the controller gains, i.e. the controller gains that were computed for Operating Point I, are unchanged. Only parameters in the controllers that change with a change in the operating condition are the desired reference values of the state variables and the outputs. In all three operating conditions the desired steady state operating value of the frequency $\omega$ is 1 p.u., which is evident from the differential equation, $\dot{\delta}=\omega-1$. Also the infinite bus voltage $V_{\infty}$ is 1 p.u. at all three operating conditions. At Operating Condition I, machine loading or the real power $P$ generated by the synchronous generator is 1 p.u. at 0.85 lagging power factor conditions. At Operating Point II, the real power $P$ generated by the synchronous generator is 0.6368 p.u. at 0.9892 lagging power factor conditions. At Operating Point III, the real power $P$ generated by the synchronous generator is 1.3466 p.u. at 0.652 lagging power factor conditions. Thus, from Operating Point II we can see that as the power factor is increased from 0.85 to 0.9892 the real power generated by the synchronous generator decreases from 1 p.u. to 0.6368 p.u. Similarly, from Operating Condition III we can see that as the power factor is reduced to 0.652 the real power generated by the synchronous generator or the machine loading increases to 1.3466 p.u. Also the stator current $I_{a}$ of the synchronous generator which is equal to $1.0037 \mathrm{p} . \mathrm{u}$. at operating condition I, is reduced to 0.6323 p.u., when the machine loading is reduced at Operating Point II, and the stator current increases to 1.4764 p.u., when the load on the synchronous generator is increased at Operating Condition III. Thus, by varying the machine loading i.e. by increasing or decreasing the load on the generator, the operating conditions are varied.

Table 9.1: Operating Points of the SMIB

\begin{tabular}{|c|c|c|c|}
\hline & Operating Point I & Operating Point II & Operating Point III \\
\hline Variables (in p.u.) & & & -1.4281 \\
\hline$I_{d 0}$ & -0.9185 & -0.4818 & 2.37786 \\
\hline$I_{F 0}$ & 1.6315 & 1.0228 & 0 \\
\hline$I_{D 0}$ & $-4.6204 \times 10^{-6}$ & 0 & 0.37472 \\
\hline$I_{q 0}$ & 0.4047 & 0.4094 & 0 \\
\hline$I_{Q 0}$ & $5.9539 \times 10^{-5}$ & 0 & 1 \\
\hline$\omega_{0}$ & 1 & 1 & 0.88676 \\
\hline$\delta_{0}$ & 1 & 1.0325 & 1.34899 \\
\hline$T_{m 0}$ & 1.0012 & 0.6373 & 1.34899 \\
\hline$G_{V 0}$ & 1.0012 & 0.6373 & 1.2575 \\
\hline$V_{q 0}$ & 0.9670 & 0.7659 & -0.6130 \\
\hline$V_{d 0}$ & -0.6628 & -0.6710 & 1.3990 \\
\hline$V_{t 0}$ & 1.172 & 1.0182 & 1.4764 \\
\hline$V_{\infty}$ & 1.0037 & 0.6323 & 1.00 \\
\hline$\alpha$ & 1.00 & 1.00 & $3.5598^{\circ}$ \\
\hline$E_{q 0}^{\prime}$ & $3.5598^{\circ}$ & $3.5598^{\circ}$ & 1.6078 \\
\hline$\tau_{d 0}^{\prime}$ & 1.1925 & 0.8844 & 5.90 \\
\hline Stator $\operatorname{current} I_{a 0}$ & 5.90 & 5.90 & 1.3466 \\
\hline Real power $\left(P=V_{t} I_{a} \cos \phi\right)$ & 1.00 & 0.6368 & 0.652 \\
\hline power factor $(P F=\cos \phi)$ & 0.85 & 0.9892 & \\
\hline
\end{tabular}




\subsection{Simulation results for the Controllers at Operating Point II}

Figure 9.1, Figure 9.2, and Figure 9.3 show the simulation results for the LTR-based LQG controller applied to the truth model at Operating Point II. From these results we can see that the generator terminal voltage $V_{t}$ settles to a steady state value of $0.9923 \mathrm{p} . \mathrm{u}$. which is different from the desired steady state value of 1.0182 p.u., with a steady state error of 0.0259 p.u. The angular velocity $\omega$ oscillates about the desired steady state value of 1 p.u., where the oscillations decay with time. Also the rotor angle $\delta$ oscillates about a new steady state value of 1.09 p.u., which deviates from the desired steady state value of 1.0325 p.u., with a steady state error of 0.0575 p.u. Thus, we observe a small steady state error, when the LTR-based LQG controller is applied to the truth model at Operating Point II.

Figure 9.4, Figure 9.5, and Figure 9.6 show the simulation results for the nonlinear feedback linearizing controller applied to the truth model at Operating Point II. The generator terminal voltage $V_{t}$ oscillates about the desired steady state value of 1.0182 p.u., the angular velocity $\omega$ oscillates about the desired steady state value of 1 p.u., and the rotor angle $\delta$ oscillates about the desired steady state value of 1.0325 p.u. The magnitude of these oscillations are more than that seen for the LTR-based LQG controller. The transient response of the nonlinear feedback linearizing controller is not as good as the LTR-based LQG controller, but the steady state error seen in the linear controller is significantly reduced in the nonlinear controller at Operating Point II.

Figure 9.7, Figure 9.8, and Figure 9.9 show the simulation results for the LQR-based full-state feedback controller applied to the truth model at Operating Point II. The generator terminal voltage $V_{t}$ settles to the desired steady state value of $1.0182 \mathrm{p} . \mathrm{u}$. The angular velocity $\omega$ oscillates about the desired steady state value of 1 p.u., where the oscillations decay with time. Also the rotor angle $\delta$, oscillates about the desired steady state value of 1.0325 p.u. The steady state response of the LQR-based full-state feedback controller

is much better than the LTR-based LQG controller at Operating Point II. Also, the transient response of the LQR-based full-state feedback controller is better than the transient response of the nonlinear feedback linearizing controller at operating condition II. 


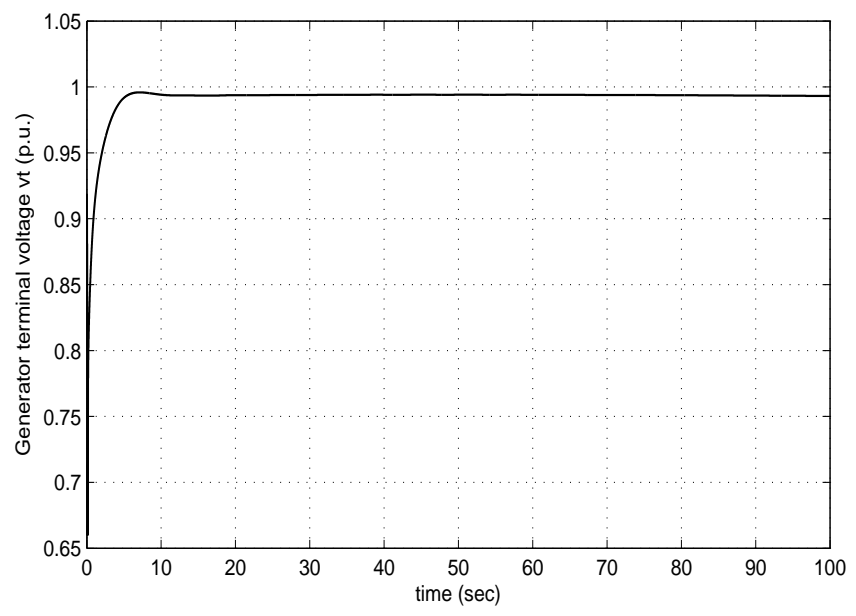

Figure 9.1: Plot of the generator terminal voltage $V_{t}$ vs time for the LTR-based LQG controller applied to the truth model (Operating Point II)

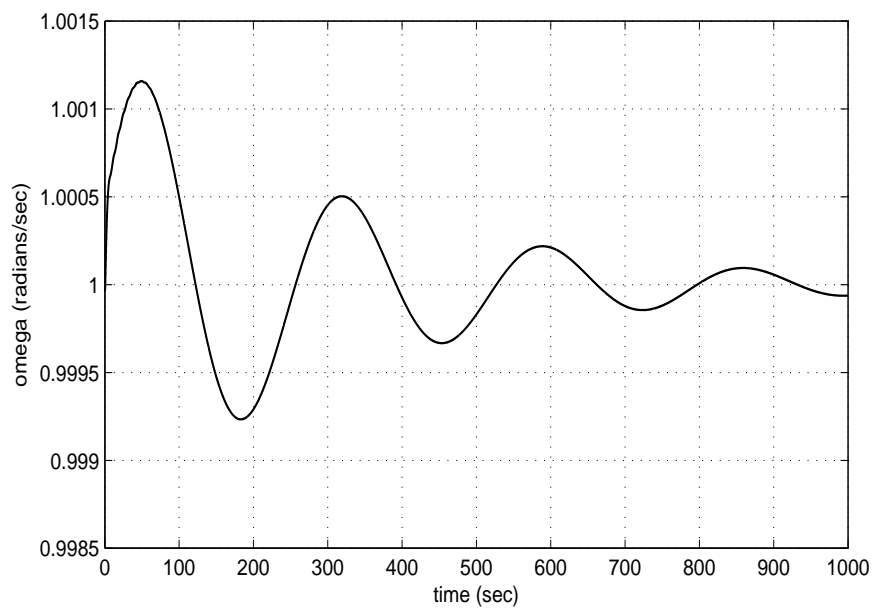

Figure 9.2: Plot of the angular velocity $\omega$ vs time for the LTR-based LQG controller applied to the truth model (Operating Point II)

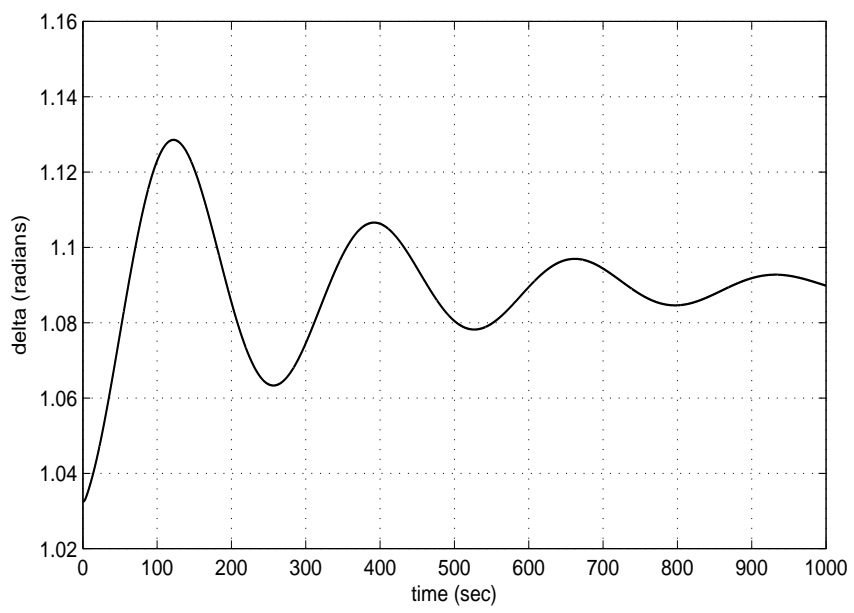

Figure 9.3: Plot of the rotor angle $\delta$ vs time for the LTR-based LQG controller applied to the truth model (Operating Point II) 


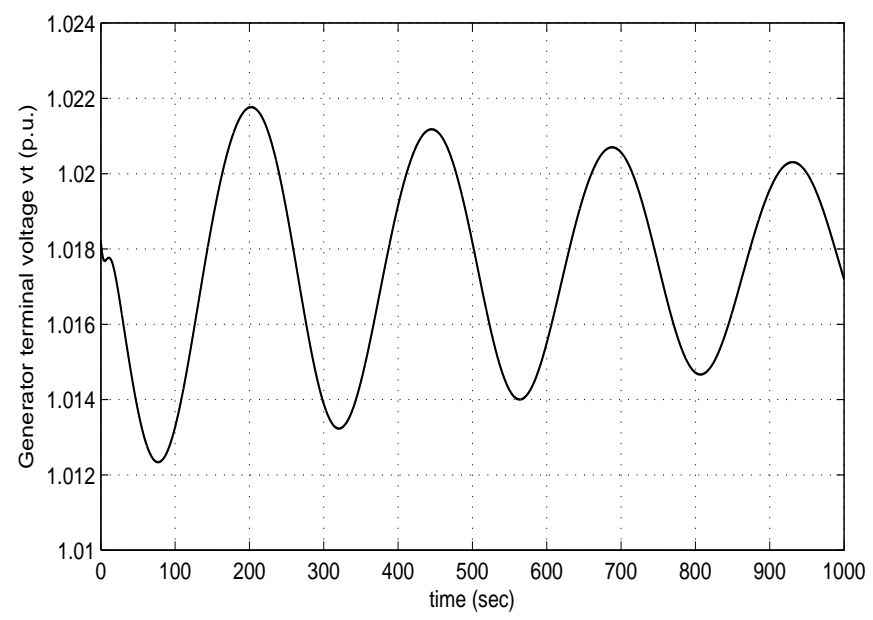

Figure 9.4: Plot of the generator terminal voltage $V_{t}$ vs time for the nonlinear feedback linearizing controller applied to the truth model (Operating Point II)

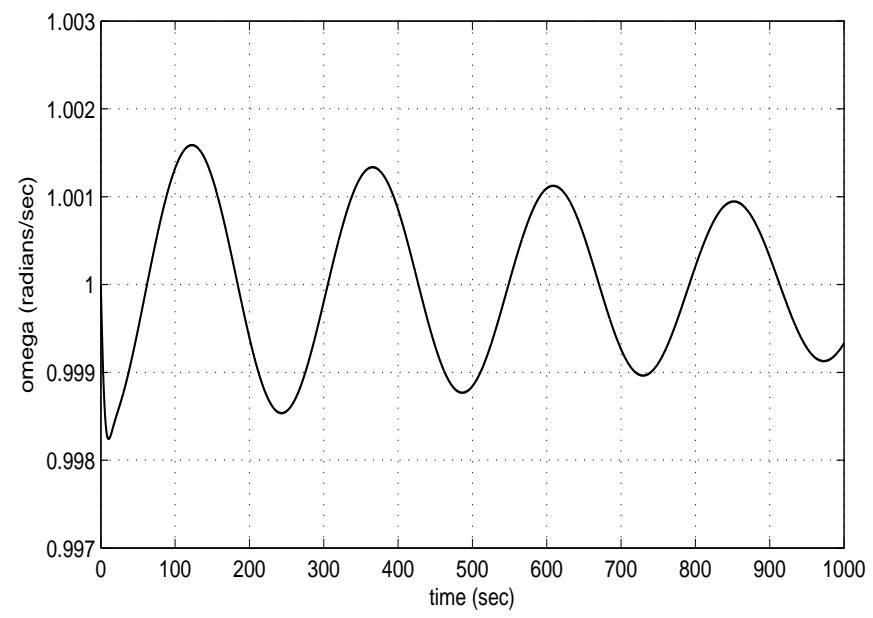

Figure 9.5: Plot of the angular velocity $\omega$ vs time for the nonlinear feedback linearizing controller applied to the truth model (Operating Point II)

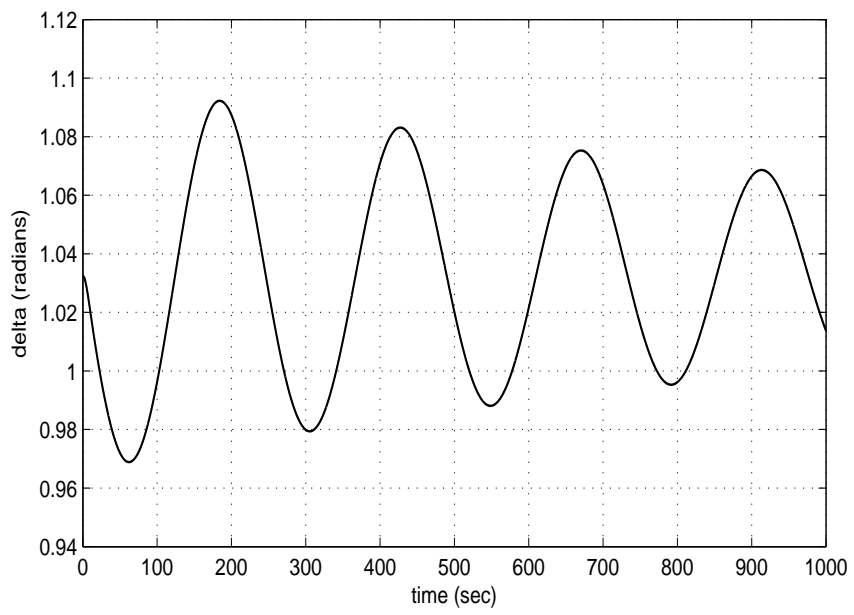

Figure 9.6: Plot of the rotor angle $\delta$ vs time for the nonlinear feedback linearizing controller applied to the truth model (Operating Point II) 


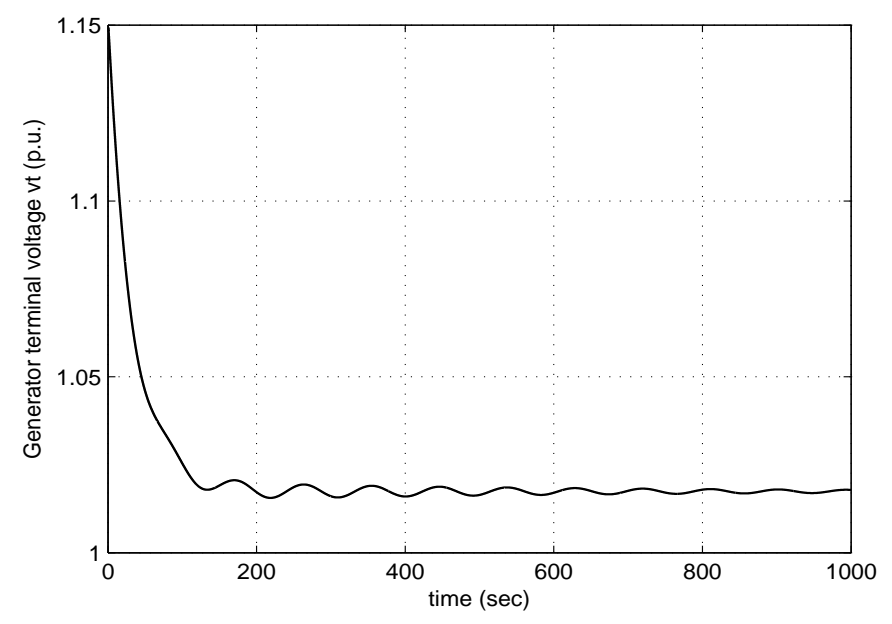

Figure 9.7: Plot of the generator terminal voltage $V_{t}$ vs time for the LQR-based full-state feedback controller applied to the truth model (Operating Point II)

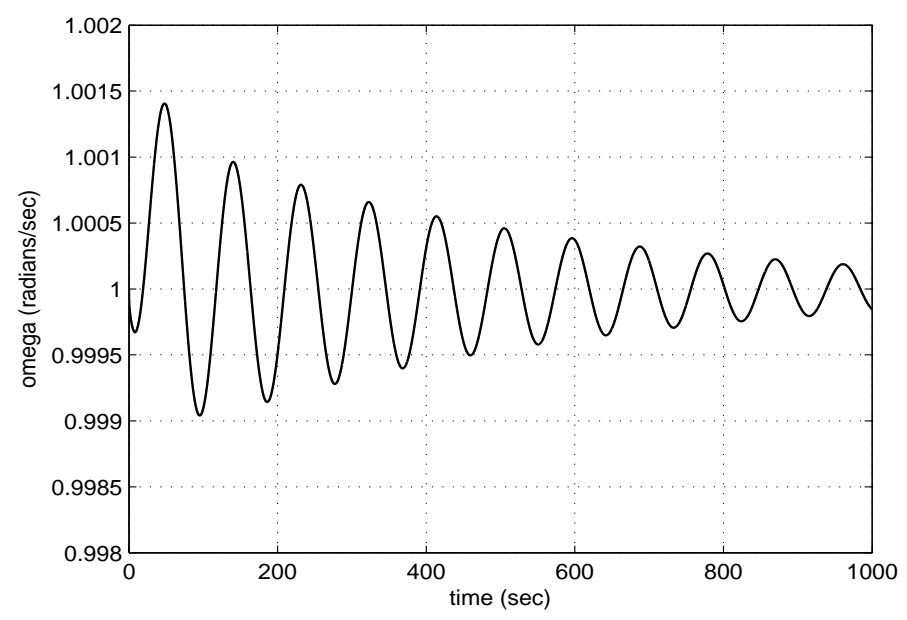

Figure 9.8: Plot of the angular velocity $\omega$ vs time for the LQR-based full-state feedback controller applied to the truth model (Operating Point II)

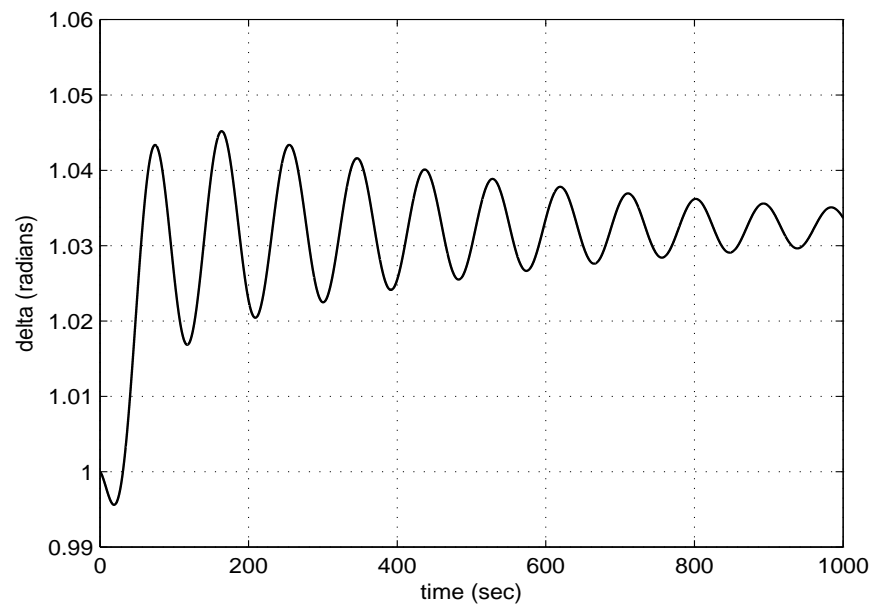

Figure 9.9: Plot of the rotor angle $\delta$ vs time for the LQR-based full-state feedback controller applied to the truth model (Operating Point II) 


\subsection{Simulation results for the Controllers at Operating Point III}

Figure 9.10, Figure 9.11, and Figure 9.12 show the simulation results for the LTR-based LQG controller applied to the truth model at Operating Point III. From these results we can see that the generator terminal voltage $V_{t}$ settles to a steady state value of $1.403 \mathrm{p}$.u. which is different from the desired steady state value of 1.3990 p.u., with a steady state error of 0.004 p.u. The angular velocity $\omega$ oscillates about the desired steady state value of 1 p.u., where the oscillations decay with time. Also the rotor angle $\delta$, oscillates about a new steady state value of 0.896 p.u. which deviates from the desired steady state value of 0.88676 p.u., with a steady state error of 0.00924 p.u. Thus, we observe a small steady state error, when the LTR-based LQG controller is applied to the truth model at Operating Point III.

Figure 9.13, Figure 9.14, and Figure 9.15 show the simulation results for the nonlinear feedback linearizing controller applied to the truth model at Operating Point III. The generator terminal voltage $V_{t}$ oscillates about the desired steady state value of 1.3990 p.u., the angular velocity $\omega$ oscillates about the desired steady state value of 1 p.u., and the rotor angle $\delta$ oscillates about the desired steady state value of 0.88676 p.u. The magnitude of these oscillations are more than that seen for the LTR-based LQG controller. The transient response of the LTR-based LQG controller is better than that of the nonlinear feedback linearizing controller, but the steady state error seen in the linear controller is significantly reduced in the nonlinear controller at operating point III.

Figure 9.16, Figure 9.17, and Figure 9.18 show the simulation results for the LQR-based full-state feedback controller applied to the truth model at Operating Point III. The generator terminal voltage $V_{t}$ settles to a steady state value of 1.3964 p.u. which is different from the desired steady state value of 1.3990 p.u., with a steady state error of 0.0026 p.u. The angular velocity $\omega$ oscillates about the desired steady state value of 1 p.u., where the oscillations decay with time. Also the rotor angle $\delta$ oscillates about a steady state value of $0.885 \mathrm{p} . \mathrm{u}$. which deviates from the desired steady state value of 0.88676 p.u., with a steady state error of 0.00176 p.u. A negligible steady state error is observed, when the LQR-based full-state feedback controller is applied to the truth model at Operating Point III. The steady state error is slightly less than that of the LTR-based LQG controller. Also the transient response of the LQR-based full-state feedback controller is better than that of the nonlinear feedback linearizing controller at operating condition III.

From these simulation results we can conclude that for small variations in the operating conditions, i.e. when the rotor angle $\delta$, is varied by a small amount from $\delta_{0}=1$ at operating point I, to $\delta_{0}=1.0325$ at operating point II, and $\delta_{0}=0.88676$ at operating point III, the transient response of the two linear controllers is better than that of the nonlinear controller, but the steady state error is the least for the nonlinear controller, followed by the LQR-based full-state feedback controller which also has a negligible steady state error compared to the LTR-based LQG controller. The controllers were also tested for large variations in the operating conditions, especially when the rotor angle is varied by a large amount from $\delta_{0}=1$ at operating point I. It is observed that as the new operating point is moved further away from operating point I, the LTR-based LQG controller applied to the truth model goes unstable, whereas the nonlinear feedback linearizing controller and the LQR-based full-state feedback controller applied to the truth model remain stable. The LQR-based full-state feedback controller shows a small steady state error, whereas the performance of the nonlinear feedback linearizing controller is independent of the operating condition. 


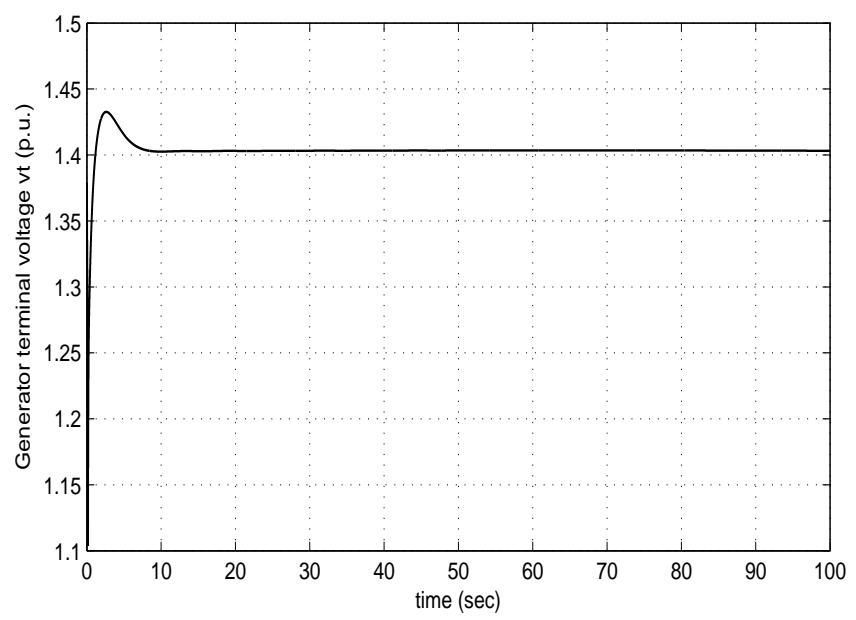

Figure 9.10: Plot of the generator terminal voltage $V_{t}$ vs time for the LTR-based LQG controller applied to the truth model (Operating Point III)

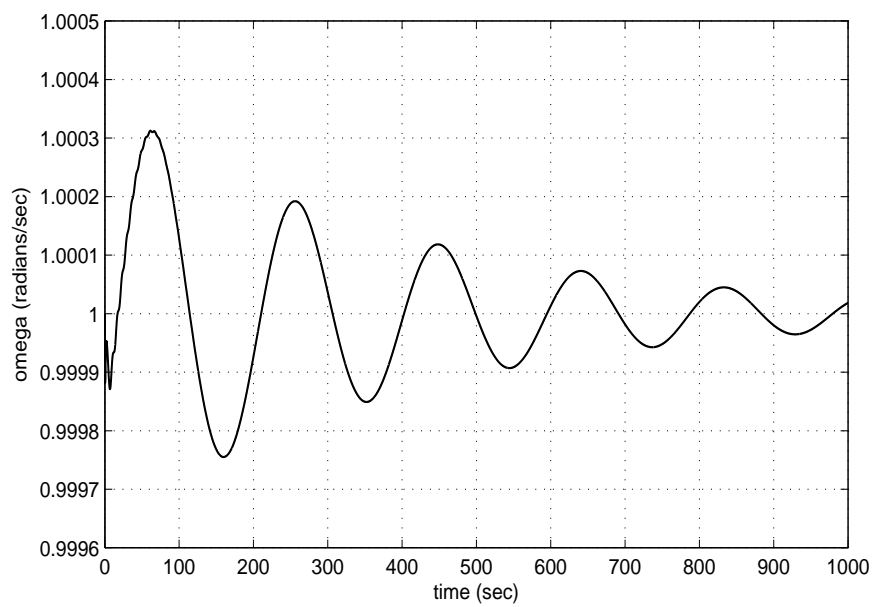

Figure 9.11: Plot of the angular velocity $\omega$ vs time for the LTR-based LQG controller applied to the truth model (Operating Point III)

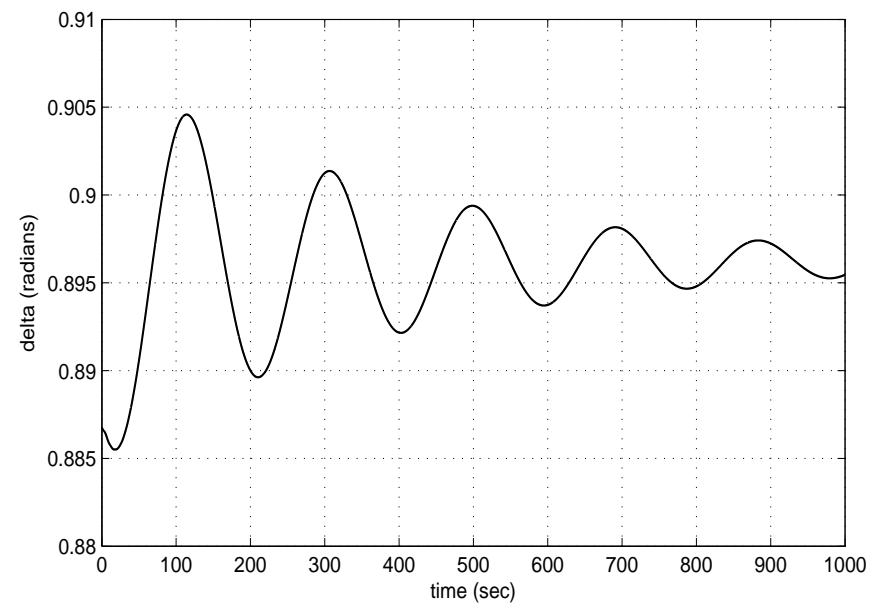

Figure 9.12: Plot of the rotor angle $\delta$ vs time for the LTR-based LQG controller applied to the truth model (Operating Point III) 


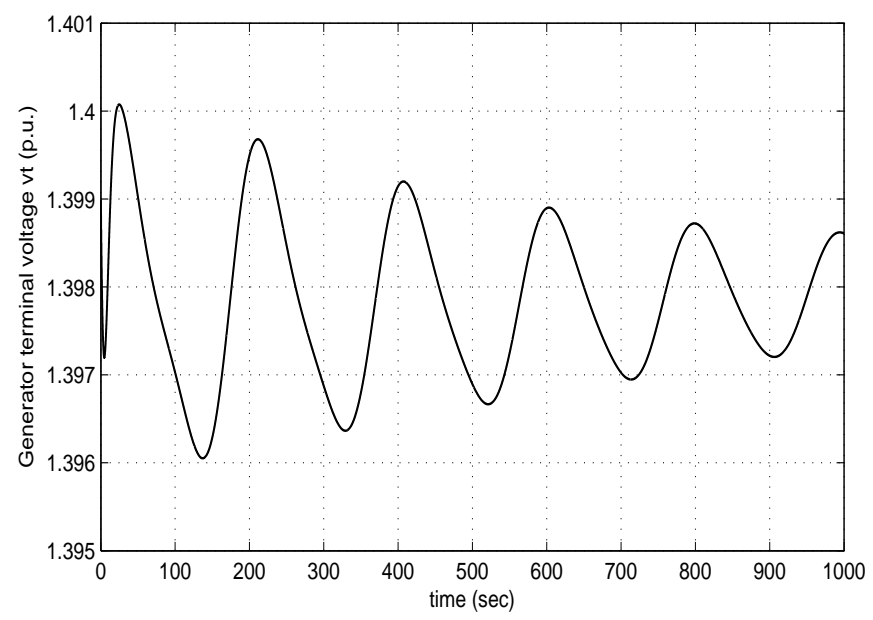

Figure 9.13: Plot of the generator terminal voltage $V_{t}$ vs time for the nonlinear feedback linearizing controller applied to the truth model (Operating Point III)

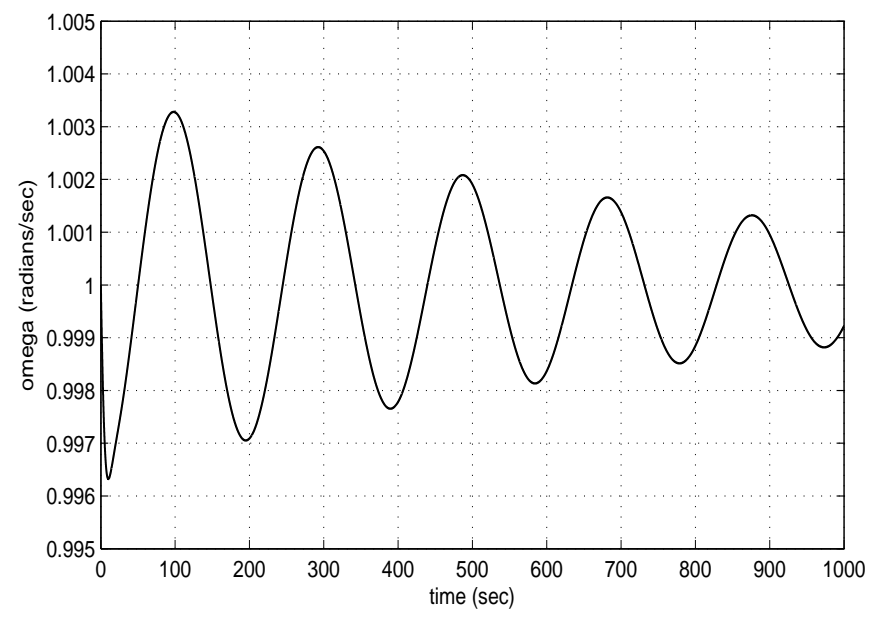

Figure 9.14: Plot of the angular velocity $\omega$ vs time for the nonlinear feedback linearizing controller applied to the truth model (Operating Point III)

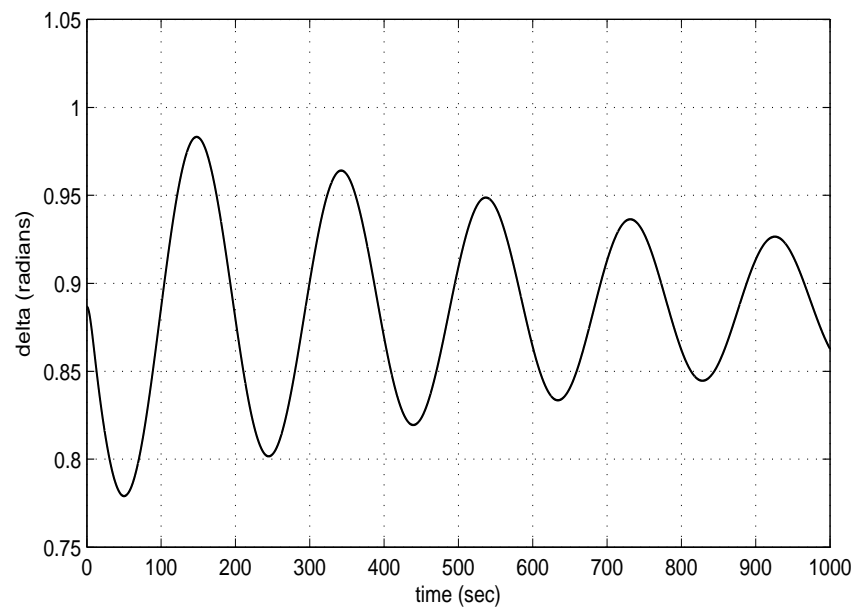

Figure 9.15: Plot of the rotor angle $\delta$ vs time for the nonlinear feedback linearizing controller applied to the truth model (Operating Point III) 


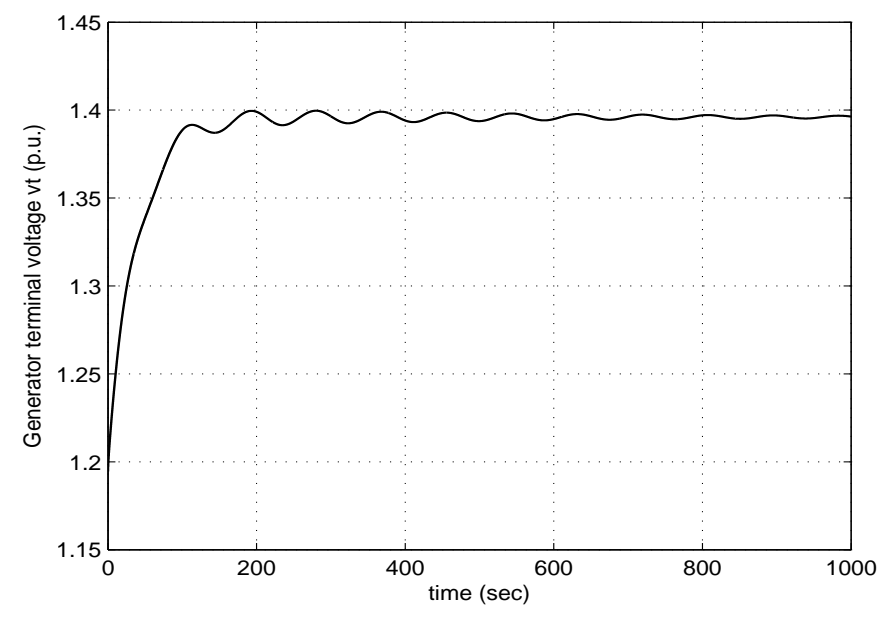

Figure 9.16: Plot of the generator terminal voltage $V_{t}$ vs time for the LQR-based full-state feedback controller applied to the truth model (Operating Point III)

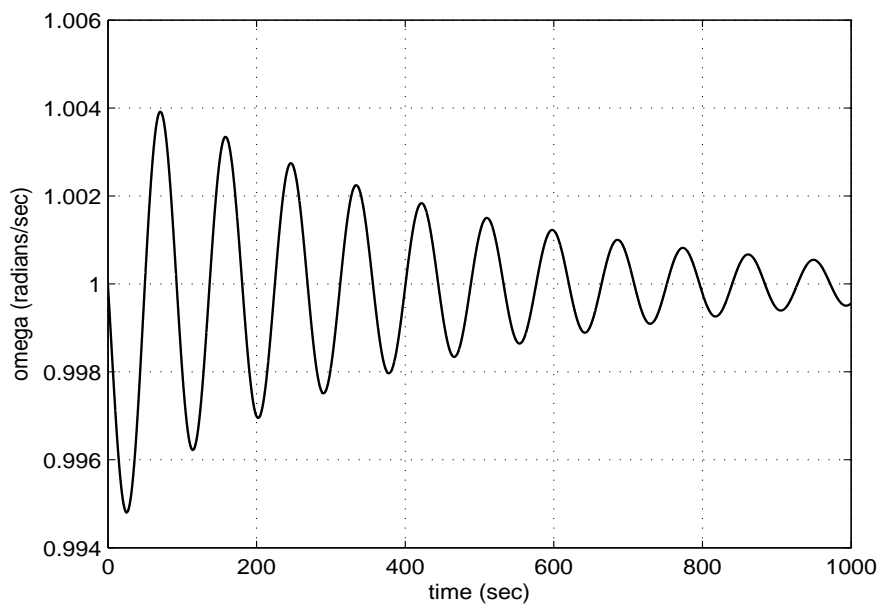

Figure 9.17: Plot of the angular velocity $\omega$ vs time for the LQR-based full-state feedback controller applied to the truth model (Operating Point III)

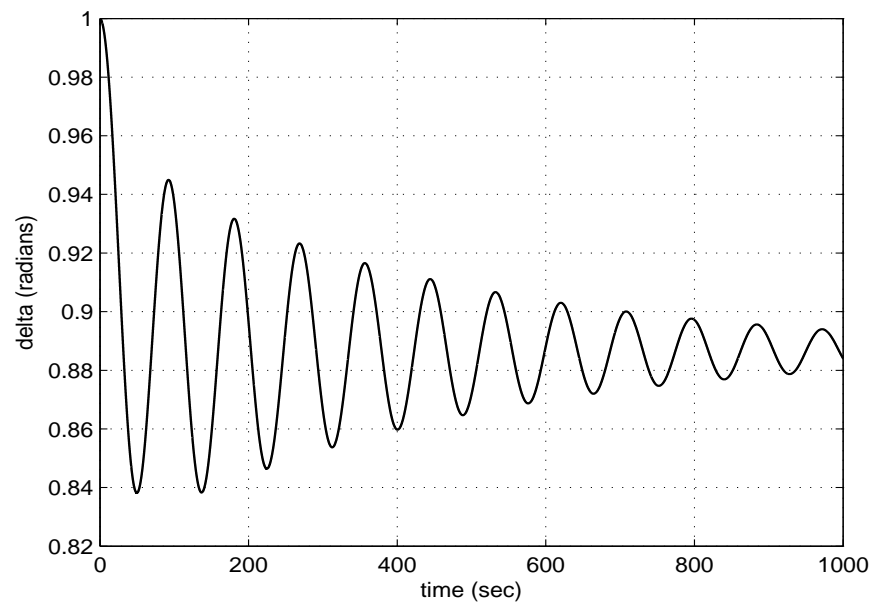

Figure 9.18: Plot of the rotor angle $\delta$ vs time for the LQR-based full-state feedback controller applied to the truth model (Operating Point III) 


\section{References}

[1] P. Anderson and A. Fouad. Power System Control and Stability. IEEE Press, 1993.

[2] A. R. Bergen and V. Vittal. Power Systems Analysis. Prentice Hall, 2000.

[3] J. C. Doyle. Robustness with observers. IEEE Transactions on Automatic Control, 1979.

[4] A. E. Fitzgerald, C. Kingsley, and S. D. Umans. Electric Machinery. McGraw-Hill Companies, Inc., 2003.

[5] A. Isidori. Nonlinear Control Systems: An Introduction. Springler-Verlag, 1989.

[6] H. K. Khalil. Nonlinear Systems. Prentice Hall, 2002.

[7] J. Machowski, J. W. Bialek, and J. R. Bumby. Power System Dynamics. John Wiley \& Sons, Ltd, 2008.

[8] H. Saadat. Power Systems Analysis. McGraw-Hill, 1999.

[9] Y. Wang, D. Hill, R. Middleton, and L. Gao. Transient stability enhancement and voltage regulation of power systems. IEEE Transactions on Power Systems, 8:620-627, May 1993. 


\section{Appendix}

\subsection{Derivation of Park's Transformation}

In this section we first present the stator and rotor inductances of a synchronous generator and then derive the voltage equation of the synchronous generator in the static frame of reference [2]. Park's transformation is then used to transform the quantities in the static frame to the rotating frame of reference. Next, we derive the voltage equation of the synchronous generator in the rotating frame of reference.

\subsubsection{Stator and Rotor Inductances}

Here, we present expressions for the self and mutual inductances of the stator and the rotor of a synchronous generator. We relate the flux linkage $\boldsymbol{\lambda}$ to the current $\mathbf{i}$ through $\boldsymbol{\lambda}=\mathbf{L}(\theta) \mathbf{i}$ assuming a linear relationship, where $\mathbf{L}(\theta)$ is a $6 \times 6$ inductance matrix relating six flux linkages to the six currents. The element $L_{i j}$ is calculated by finding the flux linkages of the $i$ th coil after setting all currents equal to zero except the current in the $j$ th coil. To simplify the analysis, we only consider the DC and the fundamental harmonic term.

The self-inductance of the stator coils are given by [2]

$$
\begin{aligned}
& L_{a a}=\frac{\lambda_{a a^{\prime}}}{i_{a}}=L_{s}+L_{m} \cos 2 \theta, L_{s}>L_{m} \geq 0 \\
& L_{b b}=\frac{\lambda_{b b^{\prime}}}{i_{b}}=L_{s}+L_{m} \cos 2\left(\theta-\frac{2 \pi}{3}\right) \\
& L_{c c}=\frac{\lambda_{c c^{\prime}}}{i_{c}}=L_{s}+L_{m} \cos 2\left(\theta+\frac{2 \pi}{3}\right)
\end{aligned}
$$

where $L_{a a}, L_{b b}$, and $L_{c c}$ denote the self-inductances of the $a, b$, and $c$ axes stator coils respectively.

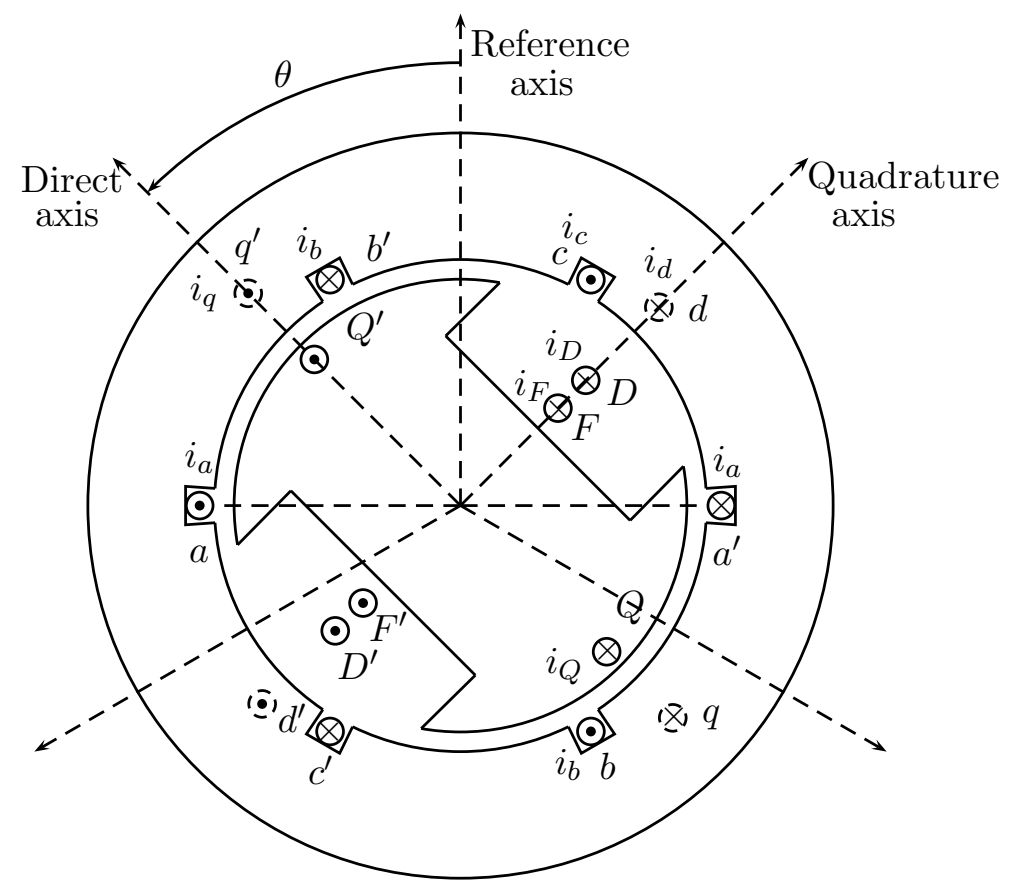

Figure 10.1: Machine schematic.

Referring to Figure 10.1, the magnetomotive force (mmf), (physical driving motive force that produces magnetic flux) due to the current in coil $a a^{\prime}$ is effective in the vertical direction. The resulting flux, with 
centerline in the vertical direction, is maximum when $\theta=0$ or $\pi$, and minimum when $\theta=\pi / 2$ or $3 \pi / 2$. The variation of $L_{a a}(\theta)$ is $\pi$-periodic. In Equation (10.1), $L_{s}$ is the DC term and $L_{m}$ is the magnitude of the fundamental harmonic term. Similar analysis is applied for $L_{b b}$ and $L_{c c}$, but phase shifts of $2 \pi / 3$ and $-2 \pi / 3$ need to be included for $L_{b b}$ and $L_{c c}$, respectively. Likewise, mutual inductances between the stator coils are given as follows,

$$
\begin{aligned}
& L_{a b}=\frac{\lambda_{a a^{\prime}}}{i_{b}}=-\left[M_{s}+L_{m} \cos 2\left(\theta+\frac{\pi}{6}\right)\right], M_{s}>L_{m} \geq 0 \\
& L_{b c}=\frac{\lambda_{b b^{\prime}}}{i_{c}}=-\left[M_{s}+L_{m} \cos 2\left(\theta-\frac{\pi}{2}\right)\right] \\
& L_{c a}=\frac{\lambda_{c c^{\prime}}}{i_{a}}=-\left[M_{s}+L_{m} \cos 2\left(\theta+\frac{5 \pi}{6}\right)\right]
\end{aligned}
$$

where $L_{a b}$ is the mutual inductance between the $a$ axis and the $b$ axis stator coils, $L_{b c}$ is the mutual inductance between the $b$ axis and the $c$ axis stator coils, $L_{c a}$ is the mutual inductance between the $c$ axis and the $a$ axis stator coils, and $M_{s}$ is the DC term of the mutual inductances. Self-inductances of the rotor coils are given by

$$
\begin{aligned}
& L_{F F}=\frac{\lambda_{F F^{\prime}}}{i_{F}}=L_{F}, L_{F}>0 \\
& L_{D D}=\frac{\lambda_{D D^{\prime}}}{i_{D}}=L_{D}, L_{D}>0 \\
& L_{Q Q}=\frac{\lambda_{Q Q^{\prime}}}{i_{Q}}=L_{Q}, L_{Q}>0
\end{aligned}
$$

where $L_{F F}, L_{D D}$, and $L_{Q Q}$ are self-inductances of the field axis, the direct axis, and the quadrature axis rotor coils respectively. Also, mutual inductances between the stator coils and the rotor coils are given as

$$
\begin{aligned}
& L_{a F}=\frac{\lambda_{a a^{\prime}}}{i_{F}}=M_{F} \cos \theta, M_{F}>0 \\
& L_{a D}=\frac{\lambda_{a a^{\prime}}}{i_{D}}=M_{D} \cos \theta, M_{D}>0 \\
& L_{a Q}=\frac{\lambda_{b b^{\prime}}}{i_{Q}}=M_{Q} \sin \theta, M_{Q}>0 \\
& L_{F D}=\frac{\lambda_{F F^{\prime}}}{i_{D}}=M_{R} \\
& L_{F Q}=\frac{\lambda_{F F^{\prime}}}{i_{Q}}=0 \\
& L_{D Q}=\frac{\lambda_{D D^{\prime}}}{i_{Q}}=0
\end{aligned}
$$

where $L_{a F}, L_{a D}$, and $L_{a Q}$ are mutual inductances between the $a$ axis stator coil and the field $F$ axis, the direct $D$ axis, and the quadrature $Q$ axis rotor coils respectively. Also $L_{F D}, L_{F Q}$, and $L_{D Q}$ are mutual inductances between the rotor coils. Like the mutual inductances between stator coils, the mutual inductances between the stator coils and the rotor coils for phase $b$ and $c$ are similar to the ones for phase $a$ which are expressed in Equation (10.4). Thus, because of symmetry the $6 \times 6$ inductance matrix is partitioned into 4 submatrices, i.e.,

$$
\mathbf{L}(\theta)=\left[\begin{array}{ll}
\mathbf{L}_{11}(\theta) & \mathbf{L}_{12}(\theta) \\
\mathbf{L}_{21}(\theta) & \mathbf{L}_{22}(\theta)
\end{array}\right]
$$


where each submatrix is expressed as

$$
\begin{aligned}
& \mathbf{L}_{11}=\left[\begin{array}{ccc}
L_{s}+L_{m} \cos 2 \theta & -M_{s}-L_{m} \cos 2\left(\theta+\frac{\pi}{6}\right) & -M_{s}-L_{m} \cos 2\left(\theta+\frac{5 \pi}{6}\right) \\
-M_{s}-L_{m} \cos 2\left(\theta+\frac{\pi}{6}\right) & L_{s}+L_{m} \cos 2\left(\theta-\frac{2 \pi}{3}\right) & -M_{s}-L_{m} \cos 2\left(\theta-\frac{\pi}{2}\right) \\
-M_{s}-L_{m} \cos 2\left(\theta+\frac{5 \pi}{6}\right) & -M_{s}-L_{m} \cos 2\left(\theta-\frac{\pi}{2}\right) & L_{s}+L_{m} \cos 2\left(\theta+\frac{2 \pi}{3}\right)
\end{array}\right] \\
& \mathbf{L}_{12}=\mathbf{L}_{21}^{\mathrm{T}}=\left[\begin{array}{ccc}
M_{F} \cos \theta & M_{D} \cos \theta & M_{Q} \sin \theta \\
M_{F} \cos \left(\theta-\frac{2 \pi}{3}\right) & M_{D} \cos \left(\theta-\frac{2 \pi}{3}\right) & M_{Q} \sin \left(\theta-\frac{2 \pi}{3}\right) \\
M_{F} \cos \left(\theta+\frac{2 \pi}{3}\right) & M_{D} \cos \left(\theta+\frac{2 \pi}{3}\right) & M_{Q} \sin \left(\theta+\frac{2 \pi}{3}\right)
\end{array}\right] \\
& \mathbf{L}_{22}=\left[\begin{array}{ccc}
L_{F} & M_{R} & 0 \\
M_{R} & L_{D} & 0 \\
0 & 0 & L_{Q}
\end{array}\right]
\end{aligned}
$$

From Equation (2.3), using $\lambda=\mathbf{L}(\theta) \mathbf{i}$, we get

$$
\mathbf{v}=-\mathbf{R i}-\frac{d \mathbf{L}(\theta)}{d t} \mathbf{i}-\mathbf{L}(\theta) \frac{d \mathbf{i}}{d t}
$$

Equation (10.7) gives the voltage equation of the synchronous generator in the static frame.

\subsubsection{Park's Transformation}

To simplify the equations and in some important cases obtain linear time-invariant equations, we introduce the zero-direct-quadrature transformation (also called Park's transformation [2], shown in Figure 10.2). By using Park's transformation, three AC quantities in the static abc frame are converted into two DC quantities in the rotating $0 d q$ frame. The rotating frame which is represented by the direct and the quadrature axis in Figure 10.2 is rotating in the same direction and has the same frequency as that of the rotor.

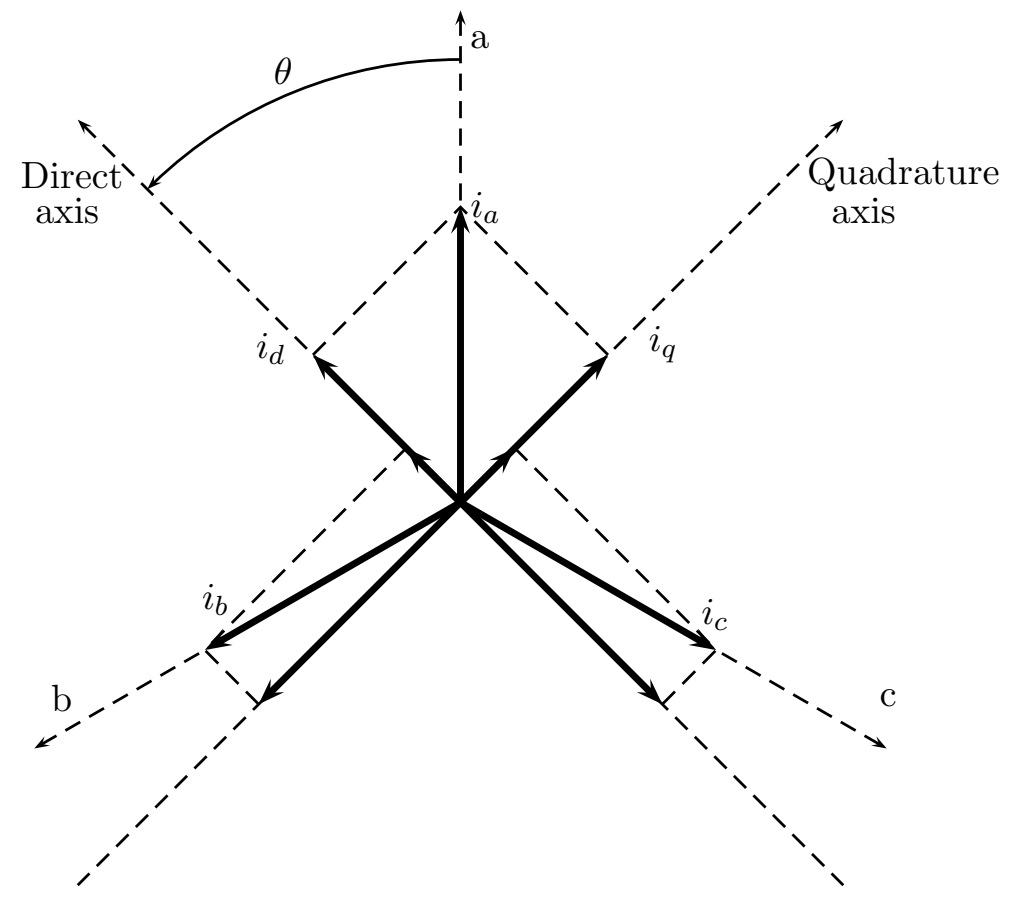

Figure 10.2: Park's Transformation 
We use the current variables to show Park's Transformation. As shown in Figure 10.2, we define the current on the direct axis as $i_{d}$ and the current on the quadrature axis as $i_{q}$. The Park's transformation is given by

$$
\begin{aligned}
i_{0} & =\sqrt{\frac{2}{3}}\left(\frac{1}{\sqrt{2}} i_{a}+\frac{1}{\sqrt{2}} i_{b}+\frac{1}{\sqrt{2}} i_{c}\right) \\
i_{d} & =\sqrt{\frac{2}{3}}\left(i_{a} \cos \theta+i_{b} \cos \left(\theta-\frac{2 \pi}{3}\right)+i_{c} \cos \left(\theta+\frac{2 \pi}{3}\right)\right) \\
i_{q} & =\sqrt{\frac{2}{3}}\left(i_{a} \sin \theta+i_{b} \sin \left(\theta-\frac{2 \pi}{3}\right)+i_{c} \sin \left(\theta+\frac{2 \pi}{3}\right)\right)
\end{aligned}
$$

where the coefficient $\sqrt{\frac{2}{3}}$ is introduced to satisfy the power conservation between the $a b c$ frame and the $0 d q$ frame. The zero-sequence component $i_{0}$, indicated by the subscript 0 , is also included.

The zero sequence component is required to yield a unique transformation of the three stator-phase quantities; it corresponds to components of armature current which produce no net air-gap flux and hence no net flux linking the rotor circuits [4]. Under balanced-three-phase conditions, there are no zero-sequence components (zero-sequence components equal 0). The Park's transformation is usually expressed in matrix form

$$
\left[\begin{array}{c}
i_{0} \\
i_{d} \\
i_{q}
\end{array}\right]=\sqrt{\frac{2}{3}}\left[\begin{array}{ccc}
\frac{1}{\sqrt{2}} & \frac{1}{\sqrt{2}} & \frac{1}{\sqrt{2}} \\
\cos \theta & \cos \left(\theta-\frac{2 \pi}{3}\right) & \cos \left(\theta+\frac{2 \pi}{3}\right) \\
\sin \theta & \sin \left(\theta-\frac{2 \pi}{3}\right) & \sin \left(\theta+\frac{2 \pi}{3}\right)
\end{array}\right]\left[\begin{array}{c}
i_{a} \\
i_{b} \\
i_{c}
\end{array}\right]
$$

We denote

$$
\mathbf{P}=\sqrt{\frac{2}{3}}\left[\begin{array}{ccc}
\frac{1}{\sqrt{2}} & \frac{1}{\sqrt{2}} & \frac{1}{\sqrt{2}} \\
\cos \theta & \cos \left(\theta-\frac{2 \pi}{3}\right) & \cos \left(\theta+\frac{2 \pi}{3}\right) \\
\sin \theta & \sin \left(\theta-\frac{2 \pi}{3}\right) & \sin \left(\theta+\frac{2 \pi}{3}\right)
\end{array}\right]
$$

It is easy to show that $\mathbf{P}$ is nonsingular and $\mathbf{P}^{-1}=\mathbf{P}^{\mathrm{T}}$.

\subsubsection{Voltage Equation in the Rotating Frame}

We first presented expressions for the self and mutual inductances of the synchronous generator and then derived the voltage equation of the synchronous generator in the static frame of reference. Then we used Park's transformation to convert the AC quantities in the static frame to the rotating frame of reference. Now we present the voltage equations of the synchronous generator in the rotating frame of reference.

If we express Equation (10.8) in matrix form, we have

$$
\mathbf{i}_{0 d q}=\mathbf{P} \mathbf{i}_{a b c}
$$

Similarly, for voltages and flux linkages, we get

$$
\begin{aligned}
\mathbf{v}_{0 d q} & =\mathbf{P} \mathbf{v}_{a b c} \\
\boldsymbol{\lambda}_{0 d q} & =\mathbf{P} \boldsymbol{\lambda}_{a b c}
\end{aligned}
$$

where the $0 d q$ terms are the ones in the rotating frame and $a b c$ terms are the ones in the static $a b c$ frame. We need to consider all six components of each current, voltage, or flux linkage vector; and we want to transform the stator-based $(a b c)$ variables into rotor-based $(0 d q)$ variables while keeping the original rotor quantities unaffected. So, we define

$$
\mathbf{i}_{B} \triangleq\left[\begin{array}{c}
i_{0} \\
i_{d} \\
i_{q} \\
\hline i_{F} \\
i_{D} \\
i_{Q}
\end{array}\right]=\left[\begin{array}{l|l}
\mathbf{P} & \mathbf{0} \\
\hline & \\
\hline \mathbf{0} & \mathbf{I}
\end{array}\right]\left[\begin{array}{c}
i_{a} \\
i_{b} \\
i_{c} \\
\hline i_{F} \\
i_{D} \\
i_{Q}
\end{array}\right]=\mathbf{B i}
$$


where $\mathbf{I}$ is the $3 \times 3$ identity matrix and $\mathbf{0}$ is the $3 \times 3$ zero matrix. Similarly, we define

$$
\begin{aligned}
\mathbf{v}_{B} & =\mathbf{B v} \\
\boldsymbol{\lambda}_{B} & =\mathbf{B} \boldsymbol{\lambda}
\end{aligned}
$$

Substituting Equation (10.12) and Equation (10.13) into $\boldsymbol{\lambda}=\mathbf{L i}$ we obtain

$$
\begin{aligned}
\mathbf{B}^{-1} \boldsymbol{\lambda}_{B} & =\mathbf{L B}^{-1} \mathbf{i}_{B} \\
\boldsymbol{\lambda}_{B} & =\mathbf{B L B}^{-1} \mathbf{i}_{B}=\mathbf{L}_{B} \mathbf{i}_{B}
\end{aligned}
$$

where $\mathbf{L}_{B} \triangleq \mathbf{B L B}^{-1}$. Using the rule for finding the transpose of a product of matrices, we find that $\mathbf{L}_{B}^{\mathrm{T}}=$ $\left(\mathbf{B L B}^{\mathrm{T}}\right)^{\mathrm{T}}=\mathbf{B L}^{\mathrm{T}} \mathbf{B}^{\mathrm{T}}=\mathbf{B L B}^{\mathrm{T}}=\mathbf{L}_{B}$. Thus $\mathbf{L}_{B}$ is symmetric because $\mathbf{L}$ is symmetric. By premultiplying by $\mathbf{B}^{-1}$ and postmultiplying by $\mathbf{B}$ we also find the relationship $\mathbf{L}=\mathbf{B}^{-1} \mathbf{L}_{B} \mathbf{B}$. It is easy to check that $\mathbf{B}^{-1}=\mathbf{B}^{\mathrm{T}}=\left[\begin{array}{cc}\mathbf{P}^{\mathrm{T}} & \mathbf{0} \\ \mathbf{0} & \mathbf{I}\end{array}\right]$. Thus we can easily calculate $\mathbf{L}_{B}$ as follows:

$$
\begin{aligned}
\mathbf{L}_{B} & =\left[\begin{array}{ll}
\mathbf{P} & \mathbf{0} \\
\mathbf{0} & \mathbf{1}
\end{array}\right]\left[\begin{array}{ll}
\mathbf{L}_{11} & \mathbf{L}_{12} \\
\mathbf{L}_{21} & \mathbf{L}_{22}
\end{array}\right]\left[\begin{array}{cc}
\mathbf{P}^{\mathrm{T}} & \mathbf{0} \\
\mathbf{0} & \mathbf{1}
\end{array}\right] \\
& =\left[\begin{array}{cc}
\mathbf{P L}_{11} \mathbf{P}^{\mathrm{T}} & \mathbf{P L}_{12} \\
\mathbf{L}_{21} \mathbf{P}^{\mathrm{T}} & \mathbf{L}_{22}
\end{array}\right]
\end{aligned}
$$

Consider now the terms in Equation (10.15). As expected, $\mathbf{L}_{22}$ is unchanged. By a tedious but straightforward calculation, we can show that

$$
\mathbf{P L}_{11} \mathbf{P}^{\mathrm{T}}=\left[\begin{array}{ccc}
L_{0} & 0 & 0 \\
0 & L_{d} & 0 \\
0 & 0 & L_{q}
\end{array}\right]
$$

where

$$
\begin{aligned}
& L_{0} \triangleq L_{s}-2 M_{s} \\
& L_{d} \triangleq L_{s}+M_{s}+\frac{3}{2} L_{m} \\
& L_{0} \triangleq L_{s}+M_{s}-\frac{3}{2} L_{m}
\end{aligned}
$$

With a knowledge of linear algebra we can show that this simplification occurs because the columns of $\mathbf{P}^{\mathrm{T}}$ are orthonormal eigenvectors of $\mathbf{L}_{11}$, and hence the similarity transformation yields a diagonal matrix of eigenvalues.

Consider next the off-diagonal submatrix $\mathbf{L}_{21} \mathbf{P}^{\mathrm{T}}=\left(\mathbf{P L}_{12}\right)^{\mathrm{T}}$. Using Equation (10.6),

$$
\mathbf{L}_{21} \mathbf{P}^{\mathrm{T}}=\left[\begin{array}{ccc}
M_{F} \cos \theta & M_{F} \cos \left(\theta-\frac{2 \pi}{3}\right) & M_{F} \cos \left(\theta+\frac{2 \pi}{3}\right) \\
M_{D} \cos \theta & M_{D} \cos \left(\theta-\frac{2 \pi}{3}\right) & M_{D} \cos \left(\theta+\frac{2 \pi}{3}\right) \\
M_{Q} \sin \theta & M_{Q} \sin \left(\theta-\frac{2 \pi}{3}\right) & M_{Q} \sin \left(\theta+\frac{2 \pi}{3}\right)
\end{array}\right] \sqrt{\frac{2}{3}}\left[\begin{array}{ccc}
\frac{1}{\sqrt{2}} & \cos \theta & \sin \theta \\
\frac{1}{\sqrt{2}} & \cos \left(\theta-\frac{2 \pi}{3}\right) & \sin \left(\theta-\frac{2 \pi}{3}\right) \\
\frac{1}{\sqrt{2}} & \cos \left(\theta+\frac{2 \pi}{3}\right) & \sin \left(\theta+\frac{2 \pi}{3}\right)
\end{array}\right]
$$

Here we recognize that the first two rows of $\mathbf{L}_{21}$ are proportional to the second column of $\mathbf{P}^{\mathrm{T}}$, which we previously indicated was orthogonal to the remaining two columns of $\mathbf{P}^{\mathrm{T}}$. The third row of $\mathbf{L}_{21}$ is proportional to the third column of $\mathbf{P}^{\mathrm{T}}$. Thus, multiplying rows into columns, it is easy to evaluate the product of the two matrices. We get

$$
\mathbf{L}_{21} \mathbf{P}^{\mathrm{T}}=\left[\begin{array}{ccc}
0 & \sqrt{\frac{3}{2}} M_{F} & 0 \\
0 & \sqrt{\frac{3}{2}} M_{D} & 0 \\
0 & 0 & \sqrt{\frac{3}{2}} M_{Q}
\end{array}\right]
$$


To simplify the notation, let $k \triangleq \sqrt{\frac{3}{2}}$; then, from Equation (10.6), Equation (10.16), Equation (10.17), and Equation (10.19), we get

$$
\mathbf{L}_{B}=\left[\begin{array}{ccc|ccc}
L_{0} & 0 & 0 & 0 & 0 & 0 \\
0 & L_{d} & 0 & k M_{F} & k M_{D} & 0 \\
0 & 0 & L_{q} & 0 & 0 & k M_{Q} \\
\hline 0 & k M_{F} & 0 & L_{F} & M_{R} & 0 \\
0 & k M_{D} & 0 & M_{R} & L_{D} & 0 \\
0 & 0 & k M_{Q} & 0 & 0 & L_{Q}
\end{array}\right]
$$

Note that the matrix $\mathbf{L}_{B}$ is simple, sparse, symmetric, and constant.

We next derive the voltage-current relations using Park's variables. Starting with Equation (2.3), which is repeated here,

$$
\mathbf{v}=-\mathbf{R i}-\frac{d \boldsymbol{\lambda}}{d t}
$$

and using Equation (10.12) and Equation (10.13), we find that

$$
\mathbf{B}^{-1} \mathbf{v}_{B}=-\mathbf{R B}^{-1} \mathbf{i}_{B}-\frac{d}{d t}\left(\mathbf{B}^{-1} \boldsymbol{\lambda}_{B}\right)
$$

Premultiplying on the left by $\mathbf{B}$, we get

$$
\mathbf{v}_{B}=-\mathbf{B R B}^{-1} \mathbf{i}_{B}-\mathbf{B} \frac{d}{d t}\left(\mathbf{B}^{-1} \boldsymbol{\lambda}_{B}\right)
$$

Using the identity

$$
\mathbf{B R B}^{-1}=\left[\begin{array}{l|l}
\mathbf{P} & \mathbf{0} \\
\hline \mathbf{0} & \mathbf{I}
\end{array}\right]\left[\begin{array}{lll|lll|l}
r & & & & \\
& r & & \mathbf{0} & \\
& & & & \\
\hline & \mathbf{0} & & r_{D} & \\
& & & & r_{Q}
\end{array}\right]\left[\begin{array}{cc|c}
\mathbf{P}^{-1} & \mathbf{0} \\
\hline & \mathbf{0} & \mathbf{I}
\end{array}\right]=\mathbf{R}
$$

We can simplify Equation (10.23) by replacing $\mathbf{B R B}^{-1}$ by $\mathbf{R}$. Continuing, and using the rule for differentiating a product, we get

$$
\mathbf{v}_{B}=-\mathbf{R} \mathbf{i}_{B}-\mathbf{B} \frac{d \mathbf{B}^{-1}}{d t} \boldsymbol{\lambda}_{B}-\frac{d \boldsymbol{\lambda}_{B}}{d t}
$$

Next, we wish to obtain a more explicit expression for the matrix $\mathbf{B} \frac{d \mathbf{B}^{-1}}{d t}$ in Equation (10.25). We first calculate $\mathbf{B} \frac{d \mathbf{B}^{-1}}{d \theta}$. Using Equation (10.12) and $\mathbf{B}^{-1}=\mathbf{B}^{\mathrm{T}}=\left[\begin{array}{cc}\mathbf{P}^{\mathrm{T}} & \mathbf{0} \\ \mathbf{0} & \mathbf{I}\end{array}\right]$, we get

$$
\mathbf{B} \frac{d \mathbf{B}^{-1}}{d \theta}=\left[\begin{array}{ll}
\mathbf{P} & \mathbf{0} \\
\mathbf{0} & \mathbf{I}
\end{array}\right]\left[\begin{array}{cc}
\frac{d \mathbf{P}^{-1}}{d \theta} & \mathbf{0} \\
\mathbf{0} & \mathbf{0}
\end{array}\right]=\left[\begin{array}{cc}
\mathbf{P} \frac{d \mathbf{P}^{-1}}{d \theta} & \mathbf{0} \\
\mathbf{0} & \mathbf{0}
\end{array}\right]
$$

where

$$
\mathbf{P} \frac{d \mathbf{P}^{-1}}{d \theta}=\frac{2}{3}\left[\begin{array}{ccc}
0 & 0 & 0 \\
0 & 0 & \frac{3}{2} \\
0 & -\frac{3}{2} & 0
\end{array}\right]=\left[\begin{array}{ccc}
0 & 0 & 0 \\
0 & 0 & 1 \\
0 & -1 & 0
\end{array}\right]
$$


Then using Equation (10.27) in Equation (10.26), we get

$$
\mathbf{B} \frac{d \mathbf{B}^{-1}}{d \theta}=\left[\begin{array}{ccc|c}
0 & 0 & 0 & \\
0 & 0 & 1 & \mathbf{0} \\
0 & -1 & 0 & \\
\hline \mathbf{0} & & \mathbf{0}
\end{array}\right]
$$

a $6 \times 6$ matrix with only two nonzero elements. Finally noting that $\mathbf{B} \frac{d \mathbf{B}^{-1}}{d t}=\mathbf{B} \frac{d \mathbf{B}^{-1}}{d \theta} \frac{d \theta}{d t}$ and substituting Equation (10.28) in Equation (10.25), we get

$$
\mathbf{v}_{B}=-\mathbf{R} \mathbf{i}_{B}-\dot{\theta}\left[\begin{array}{c}
0 \\
\lambda_{q} \\
-\lambda_{d} \\
0 \\
0 \\
0
\end{array}\right]-\frac{d \boldsymbol{\lambda}_{B}}{d t}
$$

Note 1: If the shaft position is uniform (i.e. $\dot{\theta}=\frac{d \theta}{d t}=\omega=$ constant) Equation (10.29) is linear and time invariant. Very often as a good approximation we can assume that $\dot{\theta}=$ constant.

Note 2: Although, superficially Equation (10.29) looks very much like Equation (2.3), it is important to note that Equation (10.29) is basically much simpler. In Equation (10.29),

$$
\boldsymbol{\lambda}_{B}=\mathbf{L}_{B} \mathbf{i}_{B}
$$

where $\mathbf{L}_{B}$ is the constant matrix as given in Equation (10.20), while in Equation (2.3),

$$
\lambda=\mathbf{L i}
$$

where $\mathbf{L}=\mathbf{L}(\theta)$ is a very complicated matrix as given in Equation (10.5) and Equation (10.6).

\subsection{Per Unit Conversion}

A per-unit system is the expression of system quantities as fractions of a defined base unit quantity. The stator voltages of a synchronous generator are in the kilovolt range and the field voltage is at a much lower level [1]. This problem can be solved by using the per unit conversion i.e. normalizing the equations to a convenient base value and expressing all voltages in p.u. (or percent) of base. Calculations are simplified because quantities expressed as per-unit are the same regardless of the voltage level. Generally base values of power and voltage are chosen. The base power may be the rating of a single piece of apparatus such as a motor or generator. If a system is being studied, the base power is usually chosen as a convenient round number such as 10 MVA or 100 MVA. The base voltage is chosen as the nominal rated voltage of the system. All other base quantities are derived from these two base quantities.

Let us consider an example. For a single phase system, suppose we select the base quantities of voltage [V] and apparent power [VA] as $V_{B}$ and $S_{B}$ respectively, then we can find the base quantities of current $I_{B}$ and impedance $Z_{B}$ as follows.

$$
\begin{aligned}
I_{B} & =\frac{S_{B}}{V_{B}} \\
Z_{B} & =\frac{V_{B}}{I_{B}}=\frac{V_{B}^{2}}{S_{B}}
\end{aligned}
$$

All other base quantities can be derived in a similar manner using simple laws of physics. Note: Apparent power $|S|$ is the absolute value of complex power $S$, where $S=P+j Q, P=$ real power in watt [W], and $Q$ $=$ reactive power in volt-ampere reactive [Var]. 


\subsubsection{Choosing a base for stator quantities}

The variables $v_{d}, v_{q}, i_{d}, i_{q}, \lambda_{d}$, and $\lambda_{q}$ are stator quantities because they relate directly to the $a b c$ phase quantities through Park's transformation. Using the subscript B to indicate base and $\mathrm{R}$ to indicate rated, we choose the following stator base quantities [1].

- $S_{B}=S_{R}=$ stator rated apparent power VA/phase, VA rms

- $V_{B}=V_{R}=$ stator rated line-to-neutral voltage, $\mathrm{V}$ rms

- $\omega_{B}=\omega_{R}=$ generator rated speed, elec $\mathrm{rad} / \mathrm{s}$

Note: The RMS value of a set of values (or a continuous-time waveform) is the square root of the arithmetic mean (average) of the squares of the original values (or the square of the function that defines the continuous waveform). We now develop the relations for the various base quantities. From the stator base quantities we compute the following:

- $I_{B}=\frac{S_{B}}{V_{B}}=\frac{S_{R}}{V_{R}} \quad \mathrm{~A} \mathrm{rms}$

- $t_{B}=\frac{1}{\omega_{B}}=\frac{1}{\omega_{R}} \mathrm{~s}$

- $\lambda_{B}=V_{B} t_{B}=\frac{V_{R}}{\omega_{R}}=L_{B} I_{B} \quad \mathrm{~Wb}$ turn

- $R_{B}=\frac{V_{B}}{I_{B}}=\frac{V_{R}}{I_{R}} \Omega$

- $L_{B}=\frac{V_{B} t_{B}}{I_{B}}=\frac{V_{R}}{I_{R} \omega_{R}} \mathrm{H}$

Thus by choosing the three base quantities $S_{B}, V_{B}$, and $t_{B}$, we can compute base values for all quantities of interest.

To normalize any quantity or to find its per unit value, it is divided by the base quantity of the same dimension. For example, for currents we write

$$
i_{u}=\frac{i(A)}{I_{B}(A)} \text { p.u. }
$$

where we use the subscript $u$ to indicate p.u. Later, when there is no danger of ambiguity in the notation, this subscript is omitted.

\subsubsection{Choosing a base for rotor quantities}

While choosing a base for rotor quantities, the choice of equal time base throughout all parts of a circuit with mutual coupling is the important constraint [1]. It can be shown that the choice of a common time base $t$, forces the VA base to be equal in all circuit parts and also forces the base mutual inductance to be the geometric mean of the base self-inductances if equal p.u. mutuals are to result; i.e., $M_{12 B}=\left(L_{1 B} L_{2 B}\right)^{1 / 2}$.

For the synchronous machine the choice of $S_{B}$ is based on the rating of the stator, and the time base is fixed by the rated radian frequency. These base quantities must be the same for the rotor circuits as well. It should be remembered, however, that the stator VA base is much larger than the VA rating of the rotor (field) circuits [1]. Hence some rotor base quantities are bound to be very large, making the corresponding p.u. rotor quantities appear numerically small. Therefore, care should be exercised in the choice of the remaining free rotor base term, since all other rotor base quantities will then be automatically determined. There is a choice of quantities, but the question is, Which is more convenient?

To illustrate the above, consider a machine having a stator rating of $100 \times 10^{6} \mathrm{VA} /$ phase. Assume that its exciter has a rating of $250 \mathrm{~V}$ and 1,000 A. If, for example, we choose $I_{R B}=1000 \mathrm{~A}, V_{R B}$ will then be $100,000 \mathrm{~V}$; and if we choose $V_{R B}=250 \mathrm{~V}$, then $I_{R B}$ will be $4,00,000 \mathrm{~A}$.

Is one choice more convenient than the other? Are there other more desirable choices? The answer lies in the nature of the coupling between the rotor and the stator circuits. It would seem desirable to choose 
some base quantity in the rotor to give the correct base quantity in the stator. For example, we can choose the base rotor current to give, through the magnetic coupling, the correct base stator flux linkage or open circuit voltage. Even then there is some latitude in the choice of the base rotor current, depending on the condition of the magnetic circuit.

The choice made here for the free rotor base quantity is based on the concept of equal mutual flux linkages. This means that base field current or base $d$ axis amortisseur current will produce the same space fundamental of air gap flux as produced by base stator current acting in the fictitious $d$ winding. Referring to the flux linkage equations derived in the previous section let $i_{d}=I_{B}, i_{F}=I_{F B}$, and $i_{D}=I_{D B}$ be applied one by one with other currents set to zero. If we denote the magnetizing inductances $(l=$ leakage inductances) as

$$
\begin{aligned}
L_{m d} & \triangleq L_{d}-l_{d} \mathrm{H} \\
L_{m q} & \triangleq L_{q}-l_{q} \mathrm{H} \\
L_{m F} & \triangleq L_{F}-l_{F} \mathrm{H} \\
L_{m Q} & \triangleq L_{Q}-l_{Q} \mathrm{H} \\
L_{m D} & \triangleq L_{D}-l_{D} \mathrm{H}
\end{aligned}
$$

and equate the mutual flux linkages in each winding,

$$
\begin{aligned}
\lambda_{m d} & =L_{m d} I_{B}=k M_{F} I_{F B}=k M_{D} I_{D B} \mathrm{~Wb} \\
\lambda_{m q} & =L_{m q} I_{B}=k M_{Q} I_{Q B} \mathrm{~Wb} \\
\lambda_{m F} & =k M_{F} I_{B}=L_{m F} I_{F B}=M_{R} I_{D B} \mathrm{~Wb} \\
\lambda_{m Q} & =k M_{Q} I_{B}=L_{m Q} I_{Q B} \mathrm{~Wb} \\
\lambda_{m D} & =k M_{D} I_{B}=M_{R} I_{F B}=L_{m D} I_{D B} \mathrm{~Wb}
\end{aligned}
$$

Then we can show that,

$$
\begin{aligned}
& L_{m d} I_{B}^{2}=L_{m F} I_{F B}^{2}=L_{m D} I_{D B}^{2}=k M_{F} I_{B} I_{F B}=k M_{D} I_{B} I_{D B}=M_{R} I_{F B} I_{D B} \\
& L_{m q} I_{B}^{2}=k M_{Q} I_{B} I_{Q B}=L_{m Q} I_{Q B}^{2}
\end{aligned}
$$

and this is the fundamental constraint among base currents. From the previous equation and the requirement for equal $S_{B}$, we compute

$$
\begin{aligned}
& \frac{V_{F B}}{V_{B}}=\frac{I_{B}}{I_{F B}}=\left(\frac{L_{m F}}{L_{m d}}\right)^{1 / 2}=\frac{k M_{F}}{L_{m d}}=\frac{L_{m F}}{k M_{F}}=\frac{M_{R}}{k M_{D}} \triangleq k_{F} \\
& \frac{V_{D B}}{V_{B}}=\frac{I_{B}}{I_{D B}}=\left(\frac{L_{m D}}{L_{m d}}\right)^{1 / 2}=\frac{k M_{D}}{L_{m d}}=\frac{L_{m D}}{k M_{D}}=\frac{M_{R}}{k M_{F}} \triangleq k_{D} \\
& \frac{V_{Q B}}{V_{B}}=\frac{I_{B}}{I_{Q B}}=\left(\frac{L_{m Q}}{L_{m q}}\right)^{1 / 2}=\frac{k M_{Q}}{L_{m q}}=\frac{L_{m Q}}{k M_{Q}} \triangleq k_{Q}
\end{aligned}
$$

These basic constraints permit us to compute

$$
\begin{array}{ll}
R_{F B}=k_{F}^{2} R_{B} & \Omega \\
R_{D B}=k_{D}^{2} R_{B} & \Omega \\
R_{Q B}=k_{Q}^{2} R_{B} & \Omega \\
L_{F B}=k_{F}^{2} L_{B} & \mathrm{H} \\
L_{D B}=k_{D}^{2} L_{B} & \mathrm{H} \\
L_{Q B}=k_{Q}^{2} L_{B} & \mathrm{H}
\end{array}
$$


and since the base mutuals must be the geometric mean of the base self-inductances

$$
\begin{aligned}
& M_{F B}=k_{F} L_{B} \quad \mathrm{H} \\
& M_{D B}=k_{D} L_{B} \quad \mathrm{H} \\
& M_{Q B}=k_{Q} L_{B} \quad \mathrm{H} \\
& M_{R B}=k_{F} k_{D} L_{B} \quad \mathrm{H}
\end{aligned}
$$

\subsubsection{The correspondence of per unit stator EMF to rotor quantities}

The particular choice of base quantities used here gives p.u. values of $d$ and $q$ axis stator currents and voltages that are $\sqrt{3}$ times the rms values [1]. We also note that the coupling between the $d$ axis rotor and stator involves the factor $k=\sqrt{\frac{3}{2}}$, and similarly for the q axis. For example, the contribution to the $d$ axis stator flux linkage $\lambda_{d}$ due to the field current $i_{F}$ is $k M_{F} i_{F}$ and so on. In synchronous machine equations it is often desirable to convert a rotor current, flux linkage, or voltage to an equivalent stator EMF. These expressions are developed in this section.

The basis for converting a field quantity to an equivalent stator EMF is that at open circuit a field current $i_{F} \mathrm{~A}$ corresponds to an EMF of $i_{F} \omega_{R} M_{F} \mathrm{~V}$ peak. If the rms value of this EMF is $E$, then in MKS units we have

$$
\begin{aligned}
& i_{F} \omega_{R} M_{F}=\sqrt{2} E \\
& i_{F} \omega_{R} k M_{F}=\sqrt{3} E
\end{aligned}
$$

Since $M_{F}$, and $\omega_{R}$ are known constants for a given machine, the field current corresponds to a given EMF by a simple scaling factor. Thus, $E$ is the stator air gap rms voltage in pu corresponding to the field current $i_{F}$ in pu. We can also convert a field flux linkage $\lambda_{F}$, to a corresponding stator EMF. At steady-state open circuit conditions $\lambda_{F}=L_{F} i_{F}$, and this value of field current $i_{F}$, when multiplied by $\omega_{R} M_{F}$, gives a peak stator voltage the rms value of which is denoted by $E_{q}^{\prime}$. We can show that the d axis stator EMF corresponding to the field flux linkage $\lambda_{F}$, is given by

$$
\lambda_{F} \frac{\omega_{R} k M_{F}}{L_{F}}=\sqrt{3} E_{q}^{\prime}
$$

By the same reasoning a field voltage $v_{F}$, corresponds (at steady state) to a field current $\frac{v_{F}}{r_{F}}$. This in turn corresponds to a peak stator EMF $\left(\frac{v_{F}}{r_{F}}\right) \omega_{R} M_{F}$. If the rms value of this EMF is denoted by $E_{F D}$, the $\mathrm{d}$ axis stator EMF corresponds to a field voltage $v_{F}$, or

$$
\left(\frac{v_{F}}{r_{F}}\right) \omega_{R} k M_{F}=\sqrt{3} E_{F D}
$$

\subsection{Sub-transient and Transient Inductances and Time Constants}

Before proceeding to the derivation of the simplified model of a synchronous generator we present and define some important terms. In this section we define sub-transient and transient inductances and the corresponding time constants [1] which are used in the derivation of a simplified model of a synchronous generator.

\subsubsection{Sub-transient and Transient Inductances}

If all the rotor circuits are short circuited and balanced three-phase voltages are suddenly impressed upon the stator terminals, the flux linking the $d$ axis circuit will depend initially on the sub-transient inductances, and after a few cycles on the transient inductances [1].

Let the phase voltages suddenly applied to the stator be given by

$$
\left[\begin{array}{l}
v_{a} \\
v_{b} \\
v_{c}
\end{array}\right]=\sqrt{2} V\left[\begin{array}{c}
\cos \theta \\
\cos (\theta-120) \\
\cos (\theta+120)
\end{array}\right] u(t)
$$


where $u(t)$ is a unit step function and $V$ is the rms phase voltage. Then using Park's transformation we can show that

$$
\left[\begin{array}{l}
v_{0} \\
v_{d} \\
v_{q}
\end{array}\right]=\left[\begin{array}{c}
0 \\
\sqrt{3} V u(t) \\
0
\end{array}\right]
$$

Immediately after the voltage is applied, the flux linkages $\lambda_{F}$ and $\lambda_{D}$ are still zero, since they cannot change instantly. Thus at $t=0^{+}$from Equation (2.6) we have

$$
\begin{aligned}
& \lambda_{F}=0=k M_{F} i_{d}+L_{F} i_{F}+M_{R} i_{D} \\
& \lambda_{D}=0=k M_{D} i_{d}+L_{D} i_{D}+M_{R} i_{F}
\end{aligned}
$$

Therefore

$$
\begin{aligned}
& i_{F}=-\frac{k M_{F} L_{D}-k M_{D} M_{R}}{L_{F} L_{D}-M_{R}^{2}} i_{d} \\
& i_{D}=-\frac{k M_{D} L_{F}-k M_{F} M_{R}}{L_{F} L_{D}-M_{R}^{2}} i_{d}
\end{aligned}
$$

Substituting in Equation (2.6) for $\lambda_{d}$, we get (at $t=0^{+}$)

$$
\lambda_{d}=\left(L_{d}-\frac{k^{2} M_{F}^{2} L_{D}+L_{F} k^{2} M_{D}^{2}-2 k M_{F} k M_{D} M_{R}}{L_{F} L_{D}-M_{R}^{2}}\right) i_{d}
$$

The sub-transient inductance is defined as the initial stator flux linkage per unit of stator current, with all the rotor circuits shorted and previously unenergized. Thus by definition

$$
\lambda_{d} \triangleq L_{d}^{\prime \prime} i_{d}
$$

where $L_{d}^{\prime \prime}$ is the d axis sub-transient inductance. From Equation (10.46) and Equation (10.47)

$$
\begin{aligned}
L_{d}^{\prime \prime} & =L_{d}-\frac{k^{2} M_{F}^{2} L_{D}+L_{F} k^{2} M_{D}^{2}-2 k M_{F} k M_{D} M_{R}}{L_{F} L_{D}-M_{R}^{2}} \\
L_{d}^{\prime \prime} & =L_{d}-\frac{L_{D}+L_{F}-2 L_{A D}}{\frac{L_{F} L_{D}}{L_{A D}^{2}}-1}
\end{aligned}
$$

where $L_{A D}=L_{D}-l_{D}=L_{F}-L_{f}=L_{d}-L_{d}=k M_{F}=k M_{D}=M_{R}$.

$l_{D}, l_{d}$, and $l_{F}$ are the leakage inductances of the $d, F$, and $D$ circuits respectively.

If the balanced voltages described by Equation (10.42) are suddenly applied to a machine with no damper winding, the same procedure will yield $\left(\right.$ at $\left.t=0^{+}\right)$

$$
\begin{aligned}
& i_{F}=-\frac{k M_{F}}{L_{F}} i_{d} \\
& \lambda_{d}=\left[L_{d}-\frac{\left(k M_{F}\right)^{2}}{L_{F}}\right] i_{d}=L_{d}^{\prime} i_{d}
\end{aligned}
$$

where $L_{d}^{\prime}$ is the $d$ axis transient inductance; i.e.,

$$
L_{d}^{\prime}=\left[L_{d}-\frac{\left(k M_{F}\right)^{2}}{L_{F}}\right]=L_{d}-\frac{L_{A D}^{2}}{L_{F}}
$$

In a machine with damper windings, after a few cycles from the start of the transient described in this section, the damper winding current decays rapidly to zero and the effective stator inductance is the transient inductance.

For a salient pole machine with amortisseur windings a $q$ axis damper circuit exists, but there is no other $q$ axis rotor winding. For such a machine the stator flux linkage after the initial sub-transient dies 
out is determined by essentially the same circuit as that of the steady-state $q$ axis flux linkage. Thus for a salient pole machine it is customary to consider the $q$ axis transient inductance to be the same as the $q$ axis synchronous inductance. Repeating the previous procedure for the $q$ axis circuits of a salient pole machine,

$$
\lambda_{Q}=0=k M_{Q} i_{q}+L_{Q} i_{Q}
$$

or

$$
i_{Q}=-\frac{k M_{Q}}{L_{Q}} i_{q}
$$

Substituting in the equation for $\lambda_{q}$,

$$
\lambda_{q}=L_{q} i_{q}+k M_{Q} i_{Q}
$$

or

$$
\lambda_{q}=\left[L_{q}-\frac{\left(k M_{Q}\right)^{2}}{L_{Q}}\right] i_{q} \triangleq L_{q}^{\prime \prime} i_{q}
$$

where $L_{q}^{\prime \prime}$ is the $q$ axis sub-transient inductance

$$
L_{q}^{\prime \prime}=L_{q}-\frac{\left(k M_{Q}\right)^{2}}{L_{Q}}=L_{q}-\frac{L_{A Q}^{2}}{L_{Q}}
$$

Also, for a salient pole machine the $q$ axis transient inductance $L_{q}^{\prime}$ is approximately equal to the $q$ axis synchronous inductance which is the same as $q$ axis sub-transient inductance $L_{q}^{\prime \prime}$.

$$
L_{q}^{\prime \prime}=L_{q}-\frac{\left(k M_{Q}\right)^{2}}{L_{Q}}=L_{q}^{\prime}
$$

We can also see that when $i_{Q}$ decays to zero after a few cycles, the $q$ axis effective inductance in the transient period is the same as $L_{q}$. Thus for this type of machine

$$
L_{q}^{\prime}=L_{q}
$$

Since the reactance is the product of the rated angular speed and the inductance, and since in p.u. $\omega_{R}=1$, the sub-transient and transient reactances are numerically equal to the corresponding values of inductances in (p.u.). It is important to note that for a round rotor machine $L_{q}^{\prime \prime}<L_{q}^{\prime}<L_{q}$.

\subsubsection{Time constants}

We start with the stator circuits open circuited [1]. Consider a step change in the field voltage; i.e., $v_{F}=V_{F} u(t)$. From Equation (2.5) the voltage equations can be written as

$$
\begin{aligned}
& r_{F} i_{F}+\dot{\lambda}_{F}=V_{F} u(t) \\
& r_{D} i_{D}+\dot{\lambda}_{D}=0
\end{aligned}
$$

and from Equation (2.6) the flux linkages are given by (note that $i_{d}=0$ )

$$
\begin{aligned}
& \lambda_{D}=L_{D} i_{D}+M_{R} i_{F} \\
& \lambda_{F}=L_{F} i_{F}+M_{R} i_{D}
\end{aligned}
$$

Again at $t=0^{+}, \lambda_{D}=0$, which gives for that instant

$$
i_{F}=-\left(\frac{L_{D}}{M_{R}}\right) i_{D}
$$


Substituting for the flux linkages using Equation (10.59) in Equation (10.58) we get,

$$
\begin{aligned}
\frac{V_{F}}{L_{F}} & =\left(\frac{r_{F}}{L_{F}}\right) i_{F}+\dot{i}_{F}+\left(\frac{M_{R}}{L_{F}}\right) \dot{i}_{D} \\
0 & =\left(\frac{r_{D}}{M_{R}}\right) i_{D}+\dot{i}_{F}+\left(\frac{L_{D}}{M_{R}}\right) \dot{i}_{D}
\end{aligned}
$$

Subtracting and substituting for $i_{F}$ using Equation (10.60),

$$
\dot{i}_{D}+\left(\frac{r_{D} L_{F}+r_{F} L_{D}}{L_{F} L_{D}-M_{R}^{2}}\right) i_{D}=-V_{F}\left(\frac{M_{R}}{L_{F} L_{D}-M_{R}^{2}}\right)
$$

Usually in pu $r_{D} \gg r_{F}$, while $L_{D}$ and $L_{F}$ are of similar magnitude. Therefore we can write, approximately,

$$
\dot{i}_{D}+\left(\frac{r_{D}}{L_{D}-\frac{M_{R}^{2}}{L_{F}}}\right) i_{D}=-V_{F}\left(\frac{\frac{M_{R}}{L_{F}}}{L_{D}-\frac{M_{R}^{2}}{L_{F}}}\right)
$$

Equation (10.63) shows that $i_{D}$ decays with a time constant

$$
\tau_{d 0}^{\prime \prime}=\frac{L_{D}-\frac{M_{R}^{2}}{L_{F}}}{r_{D}}
$$

This is the $d$ axis open circuit subtransient time constant. It is denoted open circuit because by definition the stator circuits are open. When the damper winding is not available or after the decay of the subtransient current, we can show that the field current is affected only by the parameters of the field circuit; i.e.,

$$
r_{F} i_{F}+L_{F} \dot{i}_{F}=V_{F} u(t)
$$

The time constant of this transient is the $d$ axis transient open circuit time constant $\tau_{d 0}^{\prime}$, where

$$
\tau_{d 0}^{\prime}=\frac{L_{F}}{r_{F}}
$$

When the stator is short circuited, the corresponding $d$ axis time constants are given by

$$
\begin{aligned}
\tau_{d}^{\prime \prime} & =\tau_{d 0}^{\prime \prime} \frac{L_{d}^{\prime \prime}}{L_{d}^{\prime}} \\
\tau_{d}^{\prime} & =\tau_{d 0}^{\prime} \frac{L_{d}^{\prime}}{L_{d}}
\end{aligned}
$$

A similar analysis of the transient in the $q$ axis circuits of a salient pole machine shows that the time constants are given by

$$
\begin{aligned}
\tau_{q 0}^{\prime \prime} & =\frac{L_{Q}}{r_{Q}} \\
\tau_{q}^{\prime \prime} & =\tau_{q 0}^{\prime \prime} \frac{L_{q}^{\prime \prime}}{L_{q}}
\end{aligned}
$$

\subsection{Simplified Model of the Synchronous Generator}

The truth model of the synchronous generator, takes into account the various effects introduced by different rotor circuits, i.e., both field effects and damper-winding effects. This model includes seven nonlinear differential equations for a single synchronous generator. In addition to these, other equations describing the load constraints, the excitation system, and the mechanical torque of the turbine-speed governor system 
must be included in the mathematical model. Thus the complete mathematical description of a large power system is exceedingly complex, and simplifications are often used in modeling the system [1].

In a stability study the response of a large number of synchronous machines to a given disturbance is investigated. The complete mathematical description of the system will therefore be very complicated unless some simplifications are used. Often only a few machines are modeled in detail, usually those nearest the disturbance, while others are described by simpler models.

In this section we first derive a two-axis simplified model of the synchronous generator and then proceed to the third order simplified model sometimes referred to in literature as the one-axis model of the synchronous generator.

\subsubsection{The two-axis model}

In the two-axis model the following assumptions are made [1]:

- Transient effects are accounted for, while the sub-transient effects are neglected. The transient effects are dominated by the rotor circuits, which are the field circuit in the $d$ axis and an equivalent circuit in the $q$ axis formed by the solid rotor.

- In the stator voltage equations the terms $\dot{\lambda}_{d}$ and $\dot{\lambda}_{q}$ are negligible compared to the speed voltage terms and that $\omega \cong \omega_{R}=1$ p.u.

- Amortisseur or damper winding effects are neglected

The machine will thus have two stator circuits and two rotor circuits. However, the number of differential equations describing these circuits is reduced by two since $\dot{\lambda}_{d}$ and $\dot{\lambda}_{q}$ are neglected in the stator voltage equations (the stator voltage equations are now algebraic equations).

The stator transient flux linkages are defined by

$$
\begin{aligned}
& \lambda_{d}^{\prime} \triangleq \lambda_{d}-L_{d}^{\prime} i_{d} \\
& \lambda_{q}^{\prime} \triangleq \lambda_{q}-L_{q}^{\prime} i_{q}
\end{aligned}
$$

and the corresponding transient stator voltages are defined by

$$
\begin{aligned}
& e_{d}^{\prime} \triangleq-\omega \lambda_{q}^{\prime}=-\omega_{R} \lambda_{q}^{\prime} \\
& e_{q}^{\prime} \triangleq \omega \lambda_{d}^{\prime}=\omega_{R} \lambda_{d}^{\prime}
\end{aligned}
$$

From Equation (2.5) the stator voltages $v_{d}$ and $v_{q}$ for the truth model of the synchronous generator are given by

$$
\begin{aligned}
& v_{d}=-r i_{d}-\omega \lambda_{q}-\frac{d \lambda_{d}}{d t} \\
& v_{q}=-r i_{q}+\omega \lambda_{d}-\frac{d \lambda_{q}}{d t}
\end{aligned}
$$

In the two-axis model we assume that in the stator voltage equations, the terms $\dot{\lambda}_{d}$ and $\dot{\lambda}_{q}$ are negligible compared to the speed voltage terms $\omega \lambda_{q}$ and $\omega \lambda_{d}$, and that $\omega \cong \omega_{R}=1$ p.u. Thus, neglecting $\frac{d \lambda_{d}}{d t}$ and $\frac{d \lambda_{q}}{d t}$ in Equation (10.71) and substituting $\omega \cong \omega_{R}$ we get

$$
\begin{aligned}
& v_{d}=-r i_{d}-\omega_{R} \lambda_{q} \\
& v_{q}=-r i_{q}+\omega_{R} \lambda_{d}
\end{aligned}
$$

Substituting $\lambda_{d}$ and $\lambda_{q}$ from Equation (10.69) in Equation (10.72) we get

$$
\begin{aligned}
v_{d} & =-r i_{d}-\omega_{R}\left(\lambda_{q}^{\prime}+L_{q}^{\prime} i_{q}\right) \\
& =-r i_{d}-\omega_{R} \lambda_{q}^{\prime}-\omega_{R} L_{q}^{\prime} i_{q} \\
v_{q} & =-r i_{q}+\omega_{R}\left(\lambda_{d}^{\prime}+L_{d}^{\prime} i_{d}\right) \\
& =-r i_{q}+\omega_{R} \lambda_{d}^{\prime}+\omega_{R} L_{d}^{\prime} i_{d}
\end{aligned}
$$


Also substituting $e_{d}^{\prime} \triangleq-\omega \lambda_{q}^{\prime}=-\omega_{R} \lambda_{q}^{\prime}$, and $e_{q}^{\prime} \triangleq \omega \lambda_{d}^{\prime}=\omega_{R} \lambda_{d}^{\prime}$ as given in Equation (10.70) into Equation (10.73) we get

$$
\begin{aligned}
& v_{d}=-r i_{d}-\omega_{R} L_{q}^{\prime} i_{q}+e_{d}^{\prime} \\
& v_{q}=-r i_{q}+\omega_{R} L_{d}^{\prime} i_{d}+e_{q}^{\prime}
\end{aligned}
$$

substituting $\omega_{R}=1$ p.u. in Equation (10.74) and rearranging we get

$$
\begin{aligned}
e_{d}^{\prime} & =v_{d}+r i_{d}+L_{q}^{\prime} i_{q} \\
& =v_{d}+r i_{d}+L_{d}^{\prime} i_{q}+\left(L_{q}^{\prime}-L_{d}^{\prime}\right) i_{q} \\
e_{q}^{\prime} & =v_{q}+r i_{q}-L_{d}^{\prime} i_{d}
\end{aligned}
$$

The above equation is in the per unit system. Since the term $\left(L_{q}^{\prime}-L_{d}^{\prime}\right) i_{q}$ is usually small, it can be neglected in Equation (10.75). Thus we can write, approximately,

$$
e_{d}^{\prime} \cong v_{d}+r i_{d}+L_{d}^{\prime} i_{q}
$$

The voltages $e_{q}^{\prime}$ and $e_{d}^{\prime}$ are the $q$ and $d$ components of a voltage $e^{\prime}$ behind the transient reactance. It is interesting to note that since $e_{d}^{\prime}$ and $e_{q}^{\prime}$ are $d$ and $q$ axis stator voltages, they represent $\sqrt{3}$ times the equivalent stator rms voltages. For example, $e_{d}^{\prime}=\sqrt{3} E_{d}^{\prime}$, and $e_{q}^{\prime}=\sqrt{3} E_{q}^{\prime}$. Also, in this model the voltage $e^{\prime}$, which corresponds to the transient flux linkages in the machine, is not a constant. Rather it will change due to the changes in the flux linkage of the $d$ and $q$ axis rotor circuits.

We now develop the differential equations for the voltages $e_{d}^{\prime}$ and $e_{q}^{\prime}$ for the two axis model. Note, all the equations given below are in per unit. The $d$ axis flux linkage equations for the truth model of a synchronous generator as given in Equation (2.6) are

$$
\left[\begin{array}{c}
\lambda_{d} \\
\lambda_{F} \\
\lambda_{D}
\end{array}\right]=\left[\begin{array}{ccc}
L_{d} & k M_{F} & k M_{D} \\
k M_{F} & L_{F} & M_{R} \\
k M_{D} & M_{R} & L_{D}
\end{array}\right]\left[\begin{array}{c}
i_{d} \\
i_{F} \\
i_{D}
\end{array}\right]
$$

i.e.

$$
\begin{aligned}
\lambda_{d} & =L_{d} i_{d}+k M_{F} i_{F}+k M_{D} i_{D} \\
\lambda_{F} & =k M_{F} i_{d}+L_{F} i_{F}+M_{R} i_{D}
\end{aligned}
$$

In the two axis model the damper winding effects are neglected. Thus, the expression for $\lambda_{D}$ and the terms $k M_{D} i_{D}$, and $M_{R} i_{D}$ in Equation (10.77) and Equation (10.78), which are related to the direct axis damper winding are neglected. Neglecting the above terms, the $d$ axis flux linkage equations for the two-axis model of a synchronous generator are

$$
\begin{aligned}
\lambda_{d} & =L_{d} i_{d}+k M_{F} i_{F} \\
\lambda_{F} & =k M_{F} i_{d}+L_{F} i_{F}
\end{aligned}
$$

The previous two equations can be solved simultaneously to compute $i_{F}$

$$
i_{F}=\frac{\lambda_{F}}{L_{F}}-\frac{k M_{F}}{L_{F}} i_{d}
$$

Substituting $i_{F}$ from Equation (10.80) in the $\lambda_{d}$ expression of Equation (10.79) we have

$$
\begin{aligned}
\lambda_{d} & =L_{d} i_{d}+k M_{F}\left(\frac{\lambda_{F}}{L_{F}}-\frac{k M_{F}}{L_{F}} i_{d}\right) \\
& =L_{d} i_{d}-\frac{\left(k M_{F}\right)^{2}}{L_{F}} i_{d}+k M_{F} \frac{\lambda_{F}}{L_{F}} \\
& =\left(L_{d}-\frac{\left(k M_{F}\right)^{2}}{L_{F}}\right) i_{d}+k M_{F} \frac{\lambda_{F}}{L_{F}}
\end{aligned}
$$


From Equation (10.50) and Equation (10.39) we have

$$
\begin{gathered}
L_{d}^{\prime}=\left(L_{d}-\frac{\left(k M_{F}\right)^{2}}{L_{F}}\right) \\
\lambda_{F} \frac{\omega_{R} k M_{F}}{L_{F}}=\sqrt{3} E_{q}^{\prime}
\end{gathered}
$$

Since all the equations are in per unit we substitute $\omega_{R}=1$ p.u. in the above expression. i.e.

$$
k M_{F} \frac{\lambda_{F}}{L_{F}}=\sqrt{3} E_{q}^{\prime}
$$

Substituting Equation (10.82) and Equation (10.84) in Equation (10.81) we get

$$
\lambda_{d}=L_{d}^{\prime} i_{d}+\sqrt{3} E_{q}^{\prime}
$$

i.e.

$$
\lambda_{d}-L_{d}^{\prime} i_{d}=\sqrt{3} E_{q}^{\prime}
$$

Using Equation (10.69) and Equation (10.70) we can verify that

$$
\lambda_{d}^{\prime} \triangleq \lambda_{d}-L_{d}^{\prime} i_{d}=\omega_{R} \lambda_{d}^{\prime}=e_{q}^{\prime}=\sqrt{3} E_{q}^{\prime}
$$

The $q$ axis flux linkage equations of the synchronous generator as given in Equation (2.7) are

$$
\left[\begin{array}{c}
\lambda_{q} \\
\lambda_{Q}
\end{array}\right]=\left[\begin{array}{cc}
L_{q} & k M_{Q} \\
k M_{Q} & L_{Q}
\end{array}\right]\left[\begin{array}{c}
i_{q} \\
i_{Q}
\end{array}\right]
$$

i.e.

$$
\begin{aligned}
\lambda_{q} & =L_{q} i_{q}+k M_{Q} i_{Q} \\
\lambda_{Q} & =k M_{Q} i_{q}+L_{Q} i_{Q}
\end{aligned}
$$

The previous two equations can be solved simultaneously to compute $i_{Q}$

$$
i_{Q}=\frac{\lambda_{Q}}{L_{Q}}-\frac{k M_{Q}}{L_{Q}} i_{q}
$$

Substituting $i_{Q}$ from Equation (10.90) in the $\lambda_{q}$ expression of Equation (10.89) we have

$$
\begin{aligned}
\lambda_{q} & =L_{q} i_{q}+k M_{Q}\left(\frac{\lambda_{Q}}{L_{Q}}-\frac{k M_{Q}}{L_{Q}} i_{q}\right) \\
& =L_{q} i_{q}-\frac{\left(k M_{Q}\right)^{2}}{L_{Q}} i_{q}+k M_{Q} \frac{\lambda_{Q}}{L_{Q}} \\
& =\left(L_{q}-\frac{\left(k M_{Q}\right)^{2}}{L_{Q}}\right) i_{q}+k M_{Q} \frac{\lambda_{Q}}{L_{Q}}
\end{aligned}
$$

From Equation (10.56) we have

$$
L_{q}^{\prime}=L_{q}-\frac{\left(k M_{Q}\right)^{2}}{L_{Q}}
$$

Substituting Equation (10.92) in Equation (10.91) we get

$$
\lambda_{q}=L_{q}^{\prime} i_{q}+k M_{Q} \frac{\lambda_{Q}}{L_{Q}}
$$

i.e.

$$
\lambda_{q}-L_{q}^{\prime} i_{q}=k M_{Q} \frac{\lambda_{Q}}{L_{Q}}
$$


Substituting $\lambda_{q}^{\prime} \triangleq \lambda_{q}-L_{q}^{\prime} i_{q}$ as given in Equation (10.69) and $e_{d}^{\prime} \triangleq-\lambda_{q}^{\prime}=-\omega_{R} \lambda_{q}^{\prime}$ as given in Equation (10.70) in Equation (10.94) we can verify that

$$
\lambda_{q}^{\prime} \triangleq \lambda_{q}-L_{q}^{\prime} i_{q}=k M_{Q} \frac{\lambda_{Q}}{L_{Q}}=\omega_{R} \lambda_{q}^{\prime}=-e_{d}^{\prime}
$$

Therefore,

$$
e_{d}^{\prime} \triangleq \sqrt{3} E_{d}^{\prime}=-\frac{k M_{Q}}{L_{Q}} \lambda_{Q}
$$

We define

$$
\begin{aligned}
\sqrt{3} E & =e_{q} \triangleq k M_{F} i_{F} \\
\sqrt{3} E_{d} & =e_{d} \triangleq-k M_{Q} i_{Q}
\end{aligned}
$$

From Equation (10.95) we have

$$
\lambda_{q}-L_{q}^{\prime} i_{q}=k M_{Q} \frac{\lambda_{Q}}{L_{Q}}=-e_{d}^{\prime}
$$

Substituting $\lambda_{q}=L_{q} i_{q}+k M_{Q} i_{Q}$ from Equation (10.89) in Equation (10.98) we get

$$
L_{q} i_{q}+k M_{Q} i_{Q}-L_{q}^{\prime} i_{q}=-e_{d}^{\prime}
$$

Substituting $\sqrt{3} E_{d}=e_{d} \triangleq-k M_{Q} i_{Q}$ from Equation (10.97) in Equation (10.99) we get

$$
L_{q} i_{q}-\sqrt{3} E_{d}-L_{q}^{\prime} i_{q}=-e_{d}^{\prime}
$$

Dividing Equation (10.100) by $\sqrt{3}$ we get

$$
L_{q} \frac{i_{q}}{\sqrt{3}}-E_{d}-L_{q}^{\prime} \frac{i_{q}}{\sqrt{3}}=-\frac{e_{d}^{\prime}}{\sqrt{3}}
$$

Converting all quantities to rms values, i.e. by substituting $\frac{i_{q}}{\sqrt{3}}=I_{q}$, and $\frac{e_{d}^{\prime}}{\sqrt{3}}=E_{d}^{\prime}$ where $I_{q}$ and $E_{d}^{\prime}$ are the rms quantities, Equation (10.101) can be written as

$$
L_{q} I_{q}-E_{d}-L_{q}^{\prime} I_{q}=-E_{d}^{\prime}
$$

i.e.

$$
E_{d}^{\prime}=E_{d}-\left(L_{q}-L_{q}^{\prime}\right) I_{q}
$$

Since the damper windings are short circuited, from the $Q$ circuit voltage equation as given in Equation (2.5) we have

$$
v_{Q}=r_{Q} i_{Q}+\frac{d \lambda_{Q}}{d t}=0
$$

Substituting $\lambda_{Q}=k M_{Q} i_{q}+L_{Q} i_{Q}$ from Equation (10.89) in Equation (10.104)

$$
v_{Q}=r_{Q} i_{Q}+k M_{Q} \dot{i}_{q}+L_{Q} \dot{i}_{Q}=0
$$

Dividing Equation (10.105) by $\sqrt{3}$ we have

$$
v_{Q}=r_{Q} \frac{i_{Q}}{\sqrt{3}}+k M_{Q} \frac{\dot{i}_{q}}{\sqrt{3}}+L_{Q} \frac{\dot{i}_{Q}}{\sqrt{3}}=0
$$

Writing $I_{Q}=\frac{i_{Q}}{\sqrt{3}}$, where $I_{Q}$ is the rms value of $i_{Q}$

$$
v_{Q}=r_{Q} I_{Q}+k M_{Q} \dot{I}_{q}+L_{Q} \dot{I}_{Q}=0
$$


Rearranging Equation (10.107) we get

$$
-\dot{I}_{Q}=\frac{r_{Q}}{L_{Q}} I_{Q}+\frac{k M_{Q}}{L_{Q}} \dot{I}_{q}
$$

From Equation (10.97) we have $\sqrt{3} E_{d}=e_{d} \triangleq-k M_{Q} i_{Q}$. Expressing all terms as rms quantities we write

$$
E_{d}=-k M_{Q} I_{Q}
$$

Differentiating Equation (10.109) we get

$$
\dot{E}_{d}=-k M_{Q} \dot{I}_{Q}
$$

Substituting $-\dot{I}_{Q}$ from Equation (10.108) in Equation (10.109)

$$
\dot{E}_{d}=k M_{Q} \frac{r_{Q}}{L_{Q}} I_{Q}+\frac{\left(k M_{Q}\right)^{2}}{L_{Q}} \dot{I}_{q}
$$

Differentiating Equation (10.103) we get

$$
\dot{E}_{d}^{\prime}=\dot{E}_{d}-\left(L_{q}-L_{q}^{\prime}\right) \dot{I}_{q}
$$

Substituting $\dot{E}_{d}$ from Equation (10.111) in Equation (10.112)

$$
\begin{aligned}
\dot{E}_{d}^{\prime} & =k M_{Q} \frac{r_{Q}}{L_{Q}} I_{Q}+\frac{\left(k M_{Q}\right)^{2}}{L_{Q}} \dot{I}_{q}-\left(L_{q}-L_{q}^{\prime}\right) \dot{I}_{q} \\
& =\frac{r_{Q}}{L_{Q}} k M_{Q} I_{Q}+\left(\frac{\left(k M_{Q}\right)^{2}}{L_{Q}}-L_{q}+L_{q}^{\prime}\right) \dot{I}_{q}
\end{aligned}
$$

Substituting $\frac{L_{Q}}{r_{Q}}=\tau_{q 0}^{\prime}, k M_{Q} I_{Q}=-E_{d}$ from Equation (10.97), and $\frac{\left(k M_{Q}\right)^{2}}{L_{Q}}-L_{q}=-L_{q}^{\prime}$ from Equation $(10.56)$ in Equation (10.113) we get

$$
\dot{E}_{d}^{\prime}=-\frac{1}{\tau_{q 0}^{\prime}} E_{d}
$$

From Equation (10.103) we have $E_{d}^{\prime}=E_{d}-\left(L_{q}-L_{q}^{\prime}\right) I_{q}$. Thus, substituting $-E_{d}=-E_{d}^{\prime}-\left(L_{q}-L_{q}^{\prime}\right) I_{q}$ in Equation (10.114)

$$
\dot{E}_{d}^{\prime}=\frac{1}{\tau_{q 0}^{\prime}}\left[-E_{d}^{\prime}-\left(L_{q}-L_{q}^{\prime}\right) I_{q}\right]
$$

The field voltage equation for the truth model of the synchronous generator as given in Equation (2.5) is

$$
v_{F}=r_{F} i_{F}+\frac{d \lambda_{F}}{d t}
$$

Substituting $\lambda_{F}=k M_{F} i_{d}+L_{F} i_{F}$ from Equation (10.79) in Equation (10.116)

$$
v_{F}=r_{F} i_{F}+k M_{F} \dot{i}_{d}+L_{F} \dot{i}_{F}
$$

From Equation (10.39), Equation (10.40), and Equation (10.41) we have

$$
\begin{aligned}
& i_{F} \omega_{R} k M_{F}=\sqrt{3} E \\
& \lambda_{F} \frac{\omega_{R} k M_{F}}{L_{F}}=\sqrt{3} E_{q}^{\prime} \\
& \left(\frac{v_{F}}{r_{F}}\right) \omega_{R} k M_{F}=\sqrt{3} E_{F D}
\end{aligned}
$$

Since all equations are in per unit, $\omega_{R}=1$. Substituting $\lambda_{F}=k M_{F} i_{d}+L_{F} i_{F}$ from Equation (10.79) in the $\sqrt{3} E_{q}^{\prime}$ expression of Equation (10.118) 


$$
\begin{aligned}
& \left(k M_{F} i_{d}+L_{F} i_{F}\right) \frac{\omega_{R} k M_{F}}{L_{F}}=\sqrt{3} E_{q}^{\prime} \\
& \frac{\omega_{R}\left(k M_{F}\right)^{2}}{L_{F}} i_{d}+i_{F} \omega_{R} k M_{F}=\sqrt{3} E_{q}^{\prime}
\end{aligned}
$$

Substituting $i_{F} \omega_{R} k M_{F}=\sqrt{3} E$ from Equation (10.118) and $\frac{\left(k M_{F}\right)^{2}}{L_{F}}=L_{d}-L_{d}^{\prime}$ from Equation (10.50) in Equation (10.119) we get

$$
\left(L_{d}-L_{d}^{\prime}\right) i_{d}+\sqrt{3} E=\sqrt{3} E_{q}^{\prime}
$$

i.e.

$$
\sqrt{3} E+L_{d} i_{d}=\sqrt{3} E_{q}^{\prime}+L_{d}^{\prime} i_{d}
$$

Multiplying Equation (10.117) by $\frac{k M_{F}}{r_{F}}$ we get

$$
\frac{v_{F}}{r_{F}} k M_{F}=i_{F} k M_{F}+\frac{\left(k M_{F}\right)^{2}}{r_{F}} \dot{i}_{d}+\frac{L_{F}}{r_{F}} k M_{F} \dot{i}_{F}
$$

Now substituting $\frac{v_{F}}{r_{F}} k M_{F}=\sqrt{3} E_{F D}, i_{F} k M_{F}=\sqrt{3} E$, and $k M_{F} \dot{i}_{F}=\sqrt{3} \dot{E}$ from Equation (10.118) in Equation (10.122) we get

$$
\sqrt{3} E_{F D}=\sqrt{3} E+\frac{\left(k M_{F}\right)^{2}}{r_{F}} \dot{i}_{d}+\frac{L_{F}}{r_{F}} \sqrt{3} \dot{E}
$$

On differentiating Equation (10.121)

$$
\sqrt{3} \dot{E}+L_{d} \dot{i}_{d}=\sqrt{3} \dot{E}_{q}^{\prime}+L_{d}^{\prime} \dot{i}_{d}
$$

Substituting $\sqrt{3} \dot{E}$ from Equation (10.124) in Equation (10.123) we get

$$
\begin{aligned}
\sqrt{3} E_{F D} & =\sqrt{3} E+\frac{\left(k M_{F}\right)^{2}}{r_{F}} \dot{i}_{d}+\frac{L_{F}}{r_{F}}\left(\sqrt{3} \dot{E}_{q}^{\prime}-L_{d} \dot{i}_{d}+L_{d}^{\prime} \dot{i}_{d}\right) \\
& =\sqrt{3} E+\frac{L_{F}}{r_{F}} \sqrt{3} \dot{E}_{q}^{\prime}+\left(\frac{\left(k M_{F}\right)^{2}}{r_{F}}+\frac{L_{F}}{r_{F}}\left(L_{d}^{\prime}-L_{d}\right)\right) \dot{i}_{d}
\end{aligned}
$$

Substituting $\frac{\left(k M_{F}\right)^{2}}{L_{F}}=L_{d}-L_{d}^{\prime}$ from Equation (10.50) in Equation (10.125)

$$
\begin{aligned}
\sqrt{3} E_{F D} & =\sqrt{3} E+\frac{L_{F}}{r_{F}} \sqrt{3} \dot{E}_{q}^{\prime}+\left(\frac{L_{F}}{r_{F}}\left(L_{d}-L_{d}^{\prime}\right)+\frac{L_{F}}{r_{F}}\left(L_{d}^{\prime}-L_{d}\right)\right) \dot{i}_{d} \\
& =\sqrt{3} E+\frac{L_{F}}{r_{F}} \sqrt{3} \dot{E}_{q}^{\prime}+0
\end{aligned}
$$

Dividing Equation (10.126) by $\sqrt{3}$ and rearranging we get

$$
\dot{E}_{q}^{\prime}=\frac{1}{\tau_{d 0}^{\prime}}\left(E_{F D}-E\right)
$$

where $\tau_{d 0}^{\prime}=\frac{L_{F}}{r_{F}}$, and $\sqrt{3} E+L_{d} i_{d}=\sqrt{3} E_{q}^{\prime}+L_{d}^{\prime} i_{d}$ i.e

$$
E=E_{q}^{\prime}-\left(L_{d}-L_{d}^{\prime}\right) I_{d}
$$

here $I_{d}$ is the rms quantity which is given by $I_{d}=\frac{i_{d}}{\sqrt{3}}$.

To complete the description of the system, the electrical torque is given by $T_{e \phi}=\lambda_{d} i_{q}-\lambda_{q} i_{d}$. Substituting $\lambda_{d}=\lambda_{d}^{\prime}+L_{d}^{\prime} i_{d}$, and $\lambda_{q}=\lambda_{q}^{\prime}+L_{q}^{\prime} i_{q}$ from Equation (10.69) we compute

$$
T_{e \phi}=\lambda_{d}^{\prime} i_{q}+L_{d}^{\prime} i_{d} i_{q}-\lambda_{q}^{\prime} i_{d}-L_{q}^{\prime} i_{q} i_{d}
$$


Substituting $e_{d}^{\prime}=-\omega_{R} \lambda_{q}^{\prime}$, and $e_{q}^{\prime}=\omega_{R} \lambda_{d}^{\prime}$ from Equation (10.70) in Equation (10.129)

$$
T_{e \phi}=e_{d}^{\prime} i_{d}+e_{q}^{\prime} i_{q}+L_{d}^{\prime} i_{d} i_{q}-L_{q}^{\prime} i_{q} i_{d}
$$

Converting all terms to rms quantities by dividing Equation (10.130) by 3

$$
\frac{T_{e \phi}}{3}=\frac{e_{d}^{\prime}}{\sqrt{3}} \frac{i_{d}}{\sqrt{3}}+\frac{e_{q}^{\prime}}{\sqrt{3}} \frac{i_{q}}{\sqrt{3}}+L_{d}^{\prime} \frac{i_{d}}{\sqrt{3}} \frac{i_{q}}{\sqrt{3}}-L_{q}^{\prime} \frac{i_{q}}{\sqrt{3}} \frac{i_{d}}{\sqrt{3}}
$$

By using $I_{q}=\frac{i_{q}}{\sqrt{3}}, I_{d}=\frac{i_{d}}{\sqrt{3}}, E_{d}^{\prime}=\frac{e_{d}^{\prime}}{\sqrt{3}}, E_{q}^{\prime}=\frac{e_{q}^{\prime}}{\sqrt{3}}$, and $T_{e}=\frac{T_{e \phi}}{\sqrt{3}}$ where $I_{q}, I_{d}, E_{d}^{\prime}, E_{q}^{\prime}$ are the rms quantities, $T_{e \phi}$ is the per unit generator electromagnetic torque defined on a per phase VA base, and $T_{e}$ is the per unit generator electromagnetic torque defined on a three-phase $(3 \phi)$ VA base. Thus, the electromagnetic torque equation of the synchronous generator is

$$
T_{e}=E_{d}^{\prime} I_{d}+E_{q}^{\prime} I_{q}-\left(L_{q}^{\prime}-L_{d}^{\prime}\right) I_{d} I_{q}
$$

From Equation (2.29), Equation (2.33), and Equation (2.34) we can write the mechanical dynamics for the two-axis model of the synchronous generator in the p.u. system as

$$
\begin{aligned}
\tau_{j} \dot{\omega} & =T_{m}-D \omega-\left(E_{d}^{\prime} I_{d}+E_{q}^{\prime} I_{q}-\left(L_{q}^{\prime}-L_{d}^{\prime}\right) I_{d} I_{q}\right) \\
\dot{\delta} & =\omega-1
\end{aligned}
$$

where $\tau_{j}$ is the time constant in per unit and is given by $\tau_{j}=2 H \omega_{R}=2 H \omega_{B}$, where $\omega_{B}=\omega_{R}=$ rated or base angular velocity of the synchronous generator. Thus, the system equations for the two-axis model of the synchronous generator consist of four differential equations which are summarized below,

$$
\begin{aligned}
\dot{E}_{d}^{\prime} & =\frac{1}{\tau_{q 0}^{\prime}}\left[-E_{d}^{\prime}-\left(L_{q}-L_{q}^{\prime}\right) I_{q}\right] \\
\dot{E}_{q}^{\prime} & =\frac{1}{\tau_{d 0}^{\prime}}\left(E_{F D}-E\right) \\
\dot{\omega} & =\frac{1}{\tau_{j}}\left[T_{m}-D \omega-\left(E_{d}^{\prime} I_{d}+E_{q}^{\prime} I_{q}-\left(L_{q}^{\prime}-L_{d}^{\prime}\right) I_{d} I_{q}\right)\right] \\
\dot{\delta} & =\omega-1
\end{aligned}
$$

\subsubsection{The one-axis or the third-order simplified model}

To obtain the third order simplified model which is also referred to in literature as the one-axis model the following assumptions are made:

- Amortisseur or damper winding effects are neglected.

- The $\dot{\lambda}_{d}$ and $\dot{\lambda}_{q}$ terms in the stator and load voltage equations are neglected compared to the speed voltage terms $\omega \lambda_{q}$ and $\omega \lambda_{d}$.

- The terms $\omega \lambda$ in the stator and load voltage equations are assumed to be approximately equal to $\omega_{R} \lambda$.

- The effect of the $Q$ circuit i.e. the differential equation for $E_{d}^{\prime}$ or $e_{d}^{\prime}$ which is a function of the current $i_{Q}$ is neglected.

It is similar to the two-axis model presented in the previous section except that the absence of the $Q$ circuit eliminates the differential equation for $E_{d}^{\prime}$ or $e_{d}^{\prime}$ which is a function of the current $i_{Q}$. The voltage behind the transient reactance $e^{\prime}$ has only the component $e_{q}^{\prime}$ changing by the field effects according to Equation (10.127). 
The component $e_{d}^{\prime}$ is completely determined from the currents and $v_{d}$. Thus, the system equations in per unit are

$$
\dot{E}_{q}^{\prime}=\frac{1}{\tau_{d 0}^{\prime}}\left(E_{F D}-E\right)
$$

where

$$
E=E_{q}^{\prime}-\left(L_{d}-L_{d}^{\prime}\right) I_{d}
$$

as given in Equation (10.128). Since the damper winding effects are neglected substituting $\dot{\lambda}_{d}=0$ in Equation (10.71), and using Equation (10.69) and Equation (10.70) we obtain the equation for $E_{d}^{\prime}$ as

$$
E_{d}^{\prime}=V_{d}+L_{q}^{\prime} I_{q}+r I_{d}
$$

From Equation (10.134) the $\dot{E}_{d}^{\prime}$ expression for the two-axis model is

$$
\dot{E}_{d}^{\prime}=\frac{1}{\tau_{q 0}^{\prime}}\left[-E_{d}^{\prime}-\left(L_{q}-L_{q}^{\prime}\right) I_{q}\right]
$$

However, in the one-axis or the third order model an additional assumption is that the differential equation for $E_{d}^{\prime}$ or $e_{d}^{\prime}$ which is a function of the current $i_{Q}$ is neglected. Thus, substituting $\dot{E}_{d}^{\prime}=0$ in Equation (10.138) we get

$$
E_{d}^{\prime}=-\left(L_{q}-L_{q}^{\prime}\right) I_{q}
$$

Equation (10.139) gives the expression for $E_{d}^{\prime}$ for the one-axis model, and it is treated as an algebraic constraint. Thus, the basic difference between the two-axis and the one-axis model is that in the two-axis model we have a differential equation for $E_{d}^{\prime}$ whereas in the one-axis model we have an algebraic expression for $E_{d}^{\prime}$. The electromagnetic torque equation for the two-axis model as given in Equation (10.132) is

$$
T_{e}=E_{d}^{\prime} I_{d}+E_{q}^{\prime} I_{q}-\left(L_{q}^{\prime}-L_{d}^{\prime}\right) I_{d} I_{q}
$$

Now substituting the algebraic constraint $E_{d}^{\prime}=-\left(L_{q}-L_{q}^{\prime}\right) I_{q}$ from Equation (10.139) in Equation (10.140) we obtain the electromagnetic torque equation for the one-axis model

$$
\begin{aligned}
T_{e} & =-\left(L_{q}-L_{q}^{\prime}\right) I_{q} I_{d}+E_{q}^{\prime} I_{q}-\left(L_{q}^{\prime}-L_{d}^{\prime}\right) I_{d} I_{q} \\
& =E_{q}^{\prime} I_{q}+\left(-L_{q}+L_{q}^{\prime}-L_{q}^{\prime}+L_{d}^{\prime}\right) I_{d} I_{q} \\
& =E_{q}^{\prime} I_{q}-\left(L_{q}-L_{d}^{\prime}\right) I_{d} I_{q}
\end{aligned}
$$

The mechanical dynamics for the one-axis model of the synchronous generator in the per unit system are

$$
\begin{aligned}
\tau_{j} \dot{\omega} & =T_{m}-D \omega-T_{e} \\
\tau_{j} \dot{\omega} & =T_{m}-D \omega-\left[E_{q}^{\prime} I_{q}-\left(L_{q}-L_{d}^{\prime}\right) I_{d} I_{q}\right] \\
\dot{\delta} & =\omega-1
\end{aligned}
$$

The system equations for the one-axis or the third order simplified model of the synchronous generator are summarized below,

$$
\begin{aligned}
\dot{E}_{q}^{\prime} & =\frac{1}{\tau_{d 0}^{\prime}}\left(E_{F D}-E\right) \\
\dot{\omega} & =\frac{1}{\tau_{j}}\left[T_{m}-D \omega-\left(E_{q}^{\prime} I_{q}-\left(L_{q}-L_{d}^{\prime}\right) I_{d} I_{q}\right)\right] \\
\dot{\delta} & =\omega-1
\end{aligned}
$$

